\title{
Effortless interactions
}

\section{for Emergency Care}

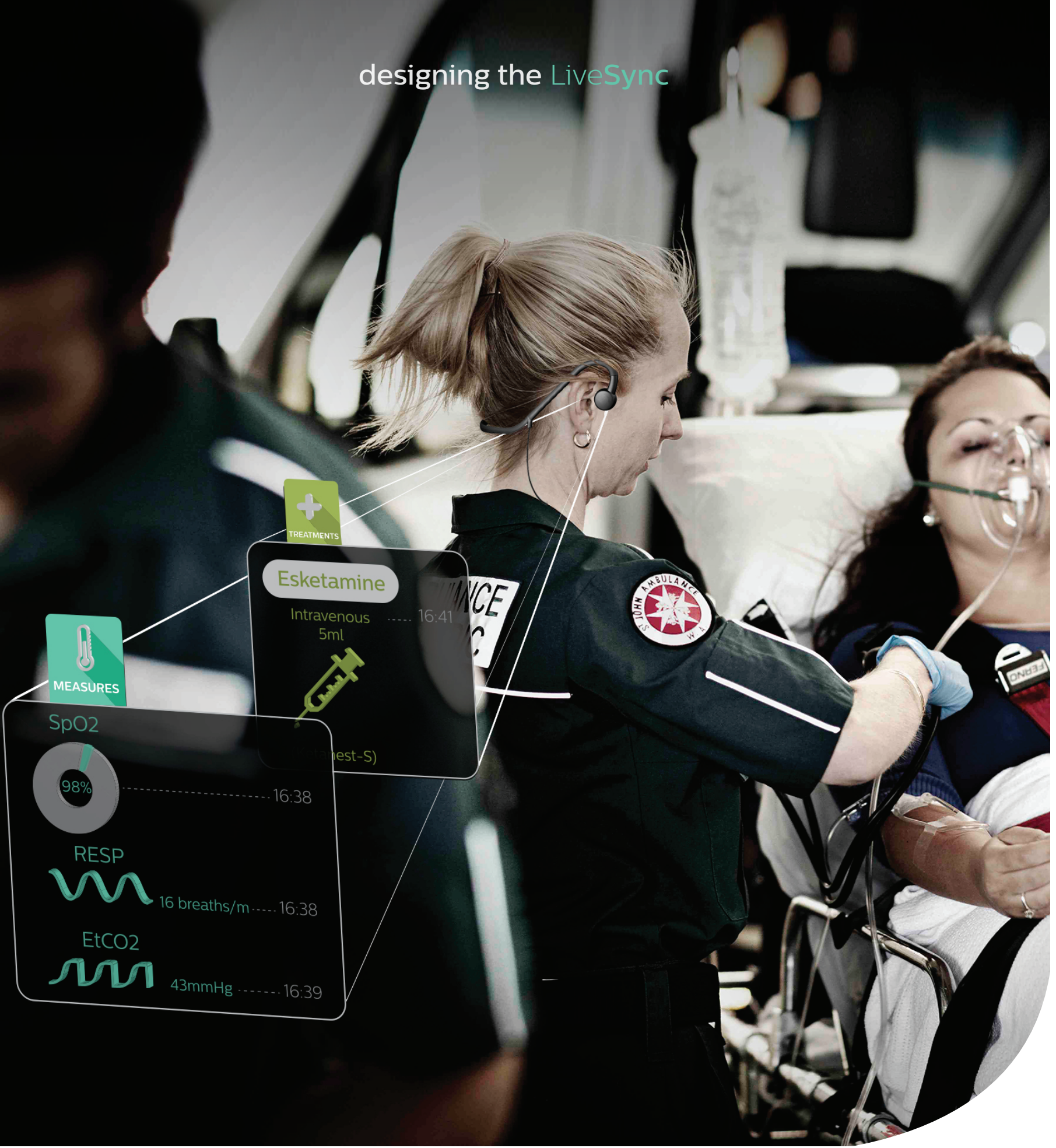




\title{
EFFORTLESS INTERACTIONS \\ FOR EMERGENCY CARE
}

\author{
Valeria Pannunzio \\ Master Thesis \\ Design for Interaction \\ Specialization Medisign \\ Valeria Pannunzio \\ Faculty of Industrial Design Engineering \\ Delft University of Technology \\ Company: Philips International B.V. \\ Company mentor: Michael Heesemans \\ Mentor: Quiel Beekman \\ Chair: Natalia Romero Herrera
}

September 2016 
"During the Industrial Age, we used machines to improve our lives by extending the capacity of our muscles During the Information Age, we are improving our lives by extending the capabilities of our minds."

McDonald, Health in the age of tele-cognition
"The practice of medicine is dominated by how we process information, how we record information, how we retrieve information, and how we communicate information " 


\title{
Thanks
}

\author{
To Natalia, Quiel and Michael for the careful, constant, and thoughtful supervision. \\ To Gavin, Jeroen, Jacco, Kiran, Christian, Igor, Joe, and the rest of the Philips Design \\ staff for the trust, the precious advices and the great atmosphere in the office. \\ To Anne-Marie, Wilma, Rob, and Rocklyn, for believing in the project and for being \\ so selflessly open: your help constituted the flesh and bones of this thesis. \\ To Nathan, Rosanne, Andreas, Hayagreev, Tanvi, for the acting, the Dutch, the \\ clothing and the business training. \\ To Bharuch, for my very psychophysical balance throughout graduation. \\ To all of the inhabitants of Heistraat 5 , for introducing me into Eindhoven's life and \\ for the late night talks. \\ To Anna, Giulia, Giuggiola, Masiello and Morera, for what Giuggiola said better than \\ me already
}

To my family, for all of the rest. 


\section{Abstract}

This thesis presents a research and The design process was characterized by a After an iterative conceptualization subsequent design intervention strong focus on analysis, and in particular phase, a final product idea was detailed developed in the context of a Design for on user research, which included intense and its interaction tested with real users
Interaction graduation assignment called

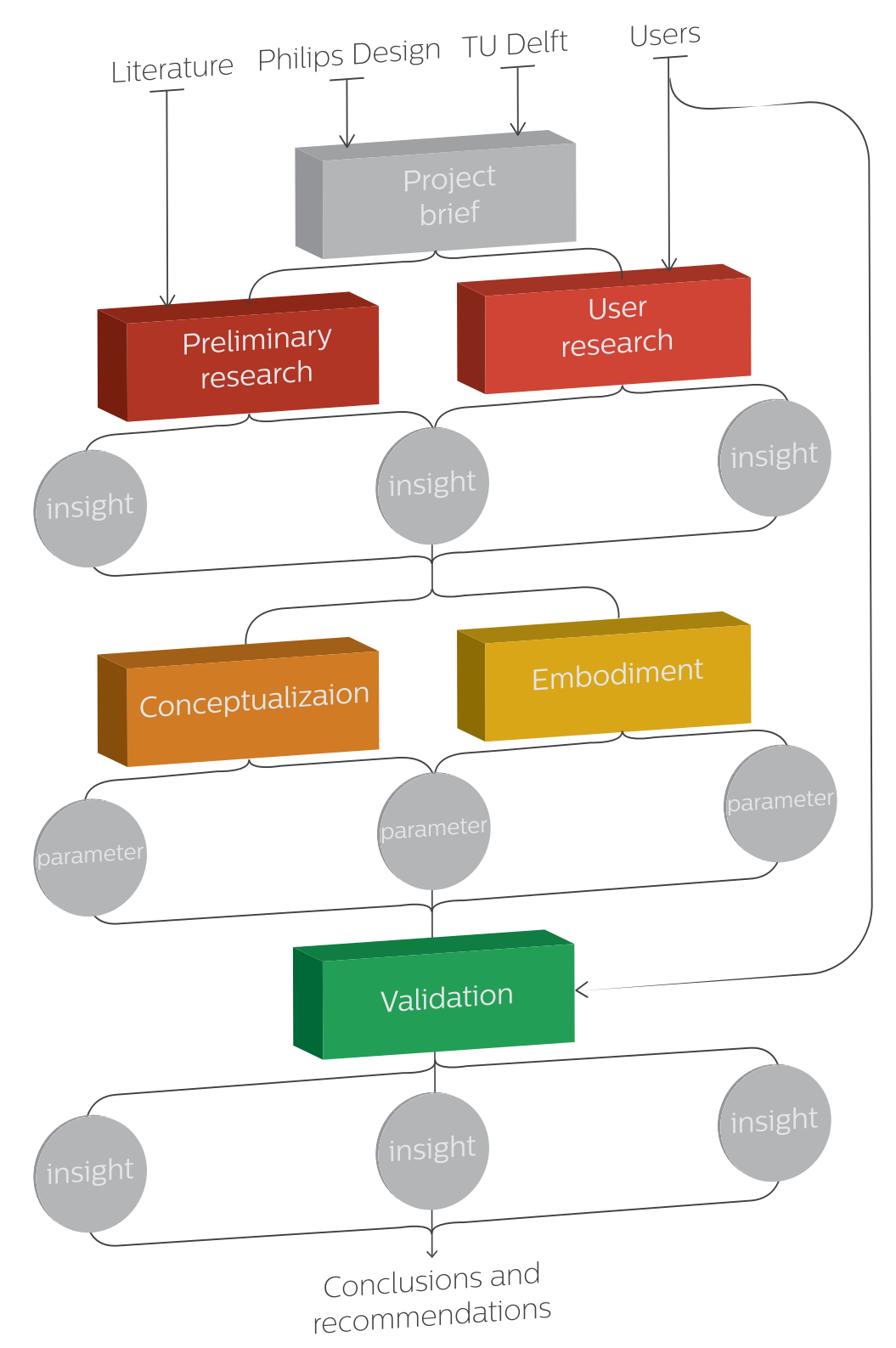




\section{Glossary and initials}

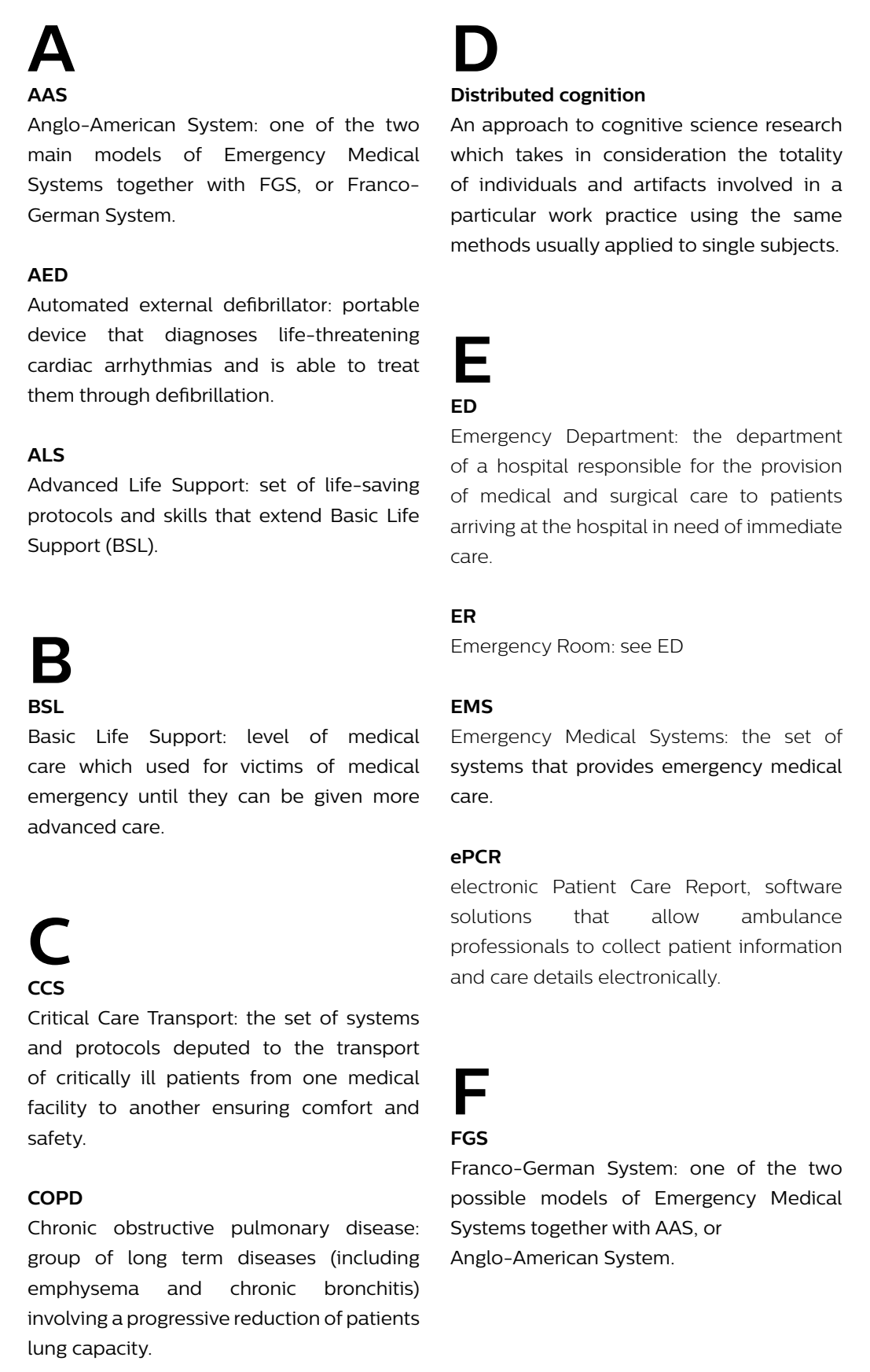

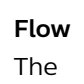
The term flow indicates, in positive dividual performing an activity is fully and did enjoyment in the accomplishment $\mathrm{T}$ Triage

The process of assessing the priority of a patient treatments based on the severity of his or her condition, for instance to determine the order and priority of patients
approaching the emergency department.

c

ent Care Report, software pofessionals to collect patient information

Chronic obstructive pulmonary disease

Franco-German System: one of the two

.

nvolving a progressive

lung capacity. 


\section{Contents}

\begin{tabular}{|c|c|c|c|}
\hline Acknowledgments & 5 & VALIDATION & 99 \\
\hline Glossary & 7 & Desirability validation & 100 \\
\hline Contents & 9 & Feasibility validation & 110 \\
\hline Introduction & 11 & Viability validation & 112 \\
\hline PRELIMINARY RESEARCH & 12 & Conclusions and recommendations & 116 \\
\hline Emergency medical services & 14 & REFLECTIONS & 118 \\
\hline EMS in the world & 18 & Reflections on research & 120 \\
\hline EMS in the Netherlands & 20 & Reflections on conceptualization & 124 \\
\hline Communication in EMS & 24 & Reflections on validation & 125 \\
\hline Teamwork in EMS & 26 & Reflections on overall process & 126 \\
\hline Trends and directions & 28 & & \\
\hline Seamless interactions & 30 & REFERENCES \& APPENDICES & 128 \\
\hline Preliminary observations & 32 & References & 130 \\
\hline USER RESEARCH & 36 & Appendix I & 132 \\
\hline Research set-up & 38 & Appendix II & 133 \\
\hline Research methodology & 40 & Appendix III & 138 \\
\hline Research results & 46 & Appendix IV & 140 \\
\hline & & Appendix V & 154 \\
\hline CONCEPTUALIZATION & 62 & Appendix VI & 156 \\
\hline Ideation process & 64 & Appendix VII & 158 \\
\hline Measures of quality & 66 & Appendix VIII & 160 \\
\hline Problem definition & 68 & Appendix IX & 162 \\
\hline Scope & 70 & Appendix X & 164 \\
\hline Creative session & 72 & Appendix XI & 166 \\
\hline Preliminary directions & 74 & Appendix XII & 168 \\
\hline Validation with users & 76 & Appendix XIII & 172 \\
\hline Direction choosing & 78 & Appendix XIV & 174 \\
\hline Future vision & 80 & Appendix XV & 182 \\
\hline EMBODIMENT & 84 & & \\
\hline Features selection & 86 & & \\
\hline Concept description & 88 & & \\
\hline Device interface & 90 & & \\
\hline Application interface & 92 & & \\
\hline Sound design & 94 & & \\
\hline Cycles of use & 96 & & \\
\hline Embodiment focus & 97 & & \\
\hline
\end{tabular}




\title{
Introduction
}

\author{
Assignment brief
}

Whenever a medical emergency eported to the 112 in Europe, a set

In case the 112 call center operato recognizes a medical emergency, ambulance will be sent to the patien

All together, the work of the ambulance operators represents an highly complex and, often, very chaotic job. This job is moreover, performed under time and possibly, emotive pressure (especially case of patients ple threat, or by nhence relatives or friends).

\section{patient conditions Estimate the need other interventions Provide the treatment or
emergency intervention Estimate the need for a transportation to a medical center communicate to the destination Emergency Department the patien conditions and personal detals conditions and if necessary kee delivering treatment.}

The last two operations, are, in particular, mostly happening simultaneously. cor words, the cractal moment information they need (to start gatherin the required professionals and equipmen in time) is expected to be executed while monitoring the patient conditions and, often, while executing essentia treatments.

This moments importance might look even more evident, considering that this constitute on the lon term a patient wil set for further medical decisions.

66

The assignment is to develop and test interaction concepts aimed at empowering emergency care staff to cope with the

high cognitive and interactional demands of their job. 
PRELIMINARY RESEARCH

This first section presents the results
of the introductory phase of the

design process. The goal of this

phase was to examine and famith
with the context of Emergency

solid theoretical framework on whic

to ground the user research. Next,

Medical Services and the main

concepts associated with it will be

provided, followed by a description
of the Dutch Emergency Medical

Service, its peculiarities, its involved developments After that a closer at the process of ambulance escuing will be given, with particular aspect. Finally, the results of a

preliminary observation conducted presented. In conclusion, a recap of the main insights contained of this section will be provided.

Emergency Medical Services
EMS in the Netherlands

Ambulance nursing
Preliminary observation 


\section{Emergency medical}

\section{services}

Context introduction
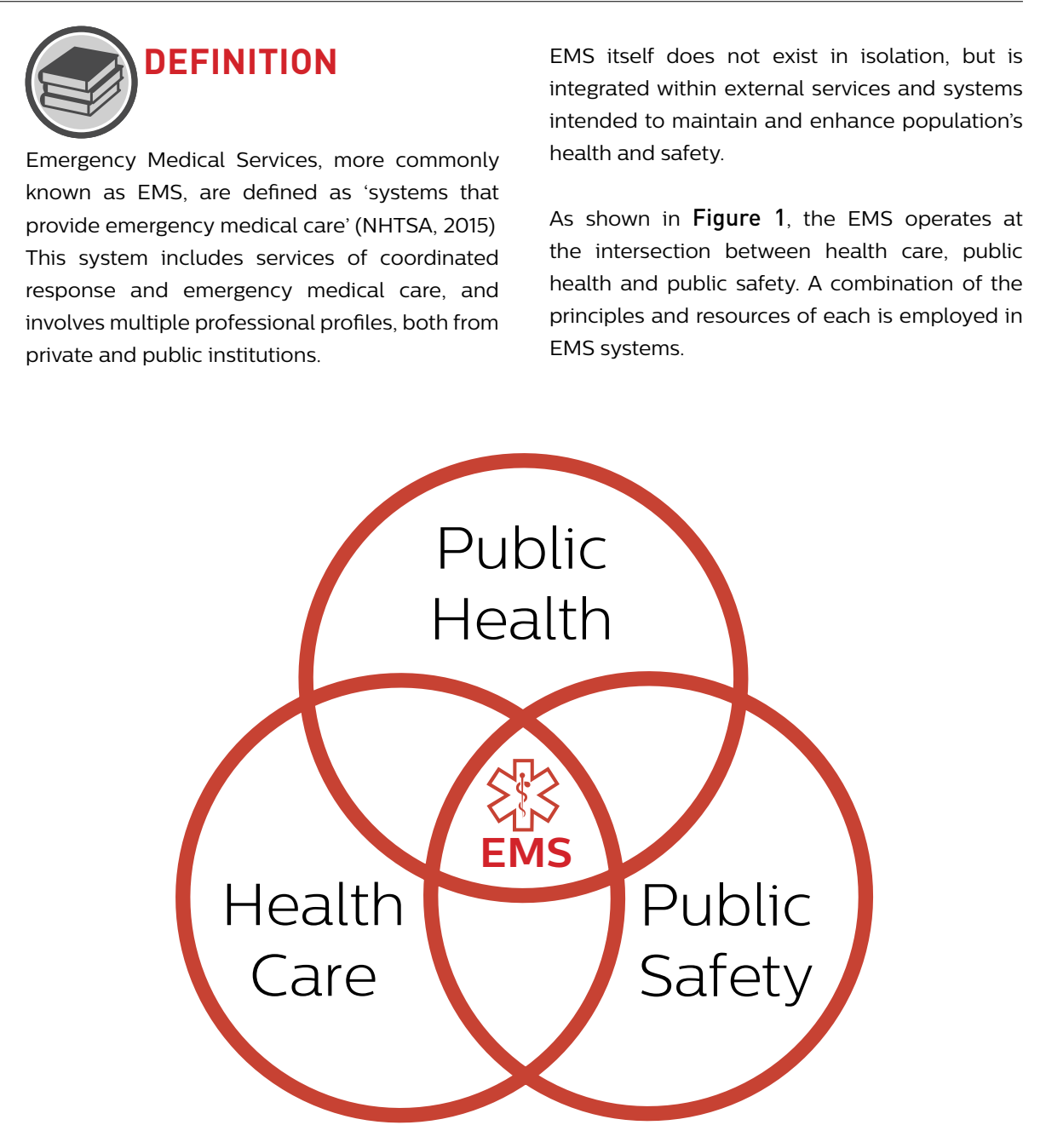

Figure 1 EMS domain
background (adapted from
NHTSA, 2015).
1. Early detection

6. Transfer to definitve care

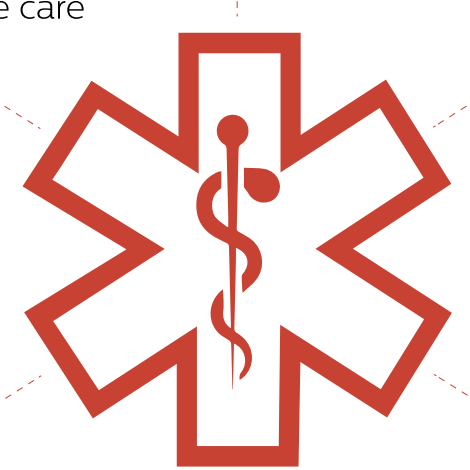

5. Care in transit

3. Early response
2. Early reporting

4. On-scene care

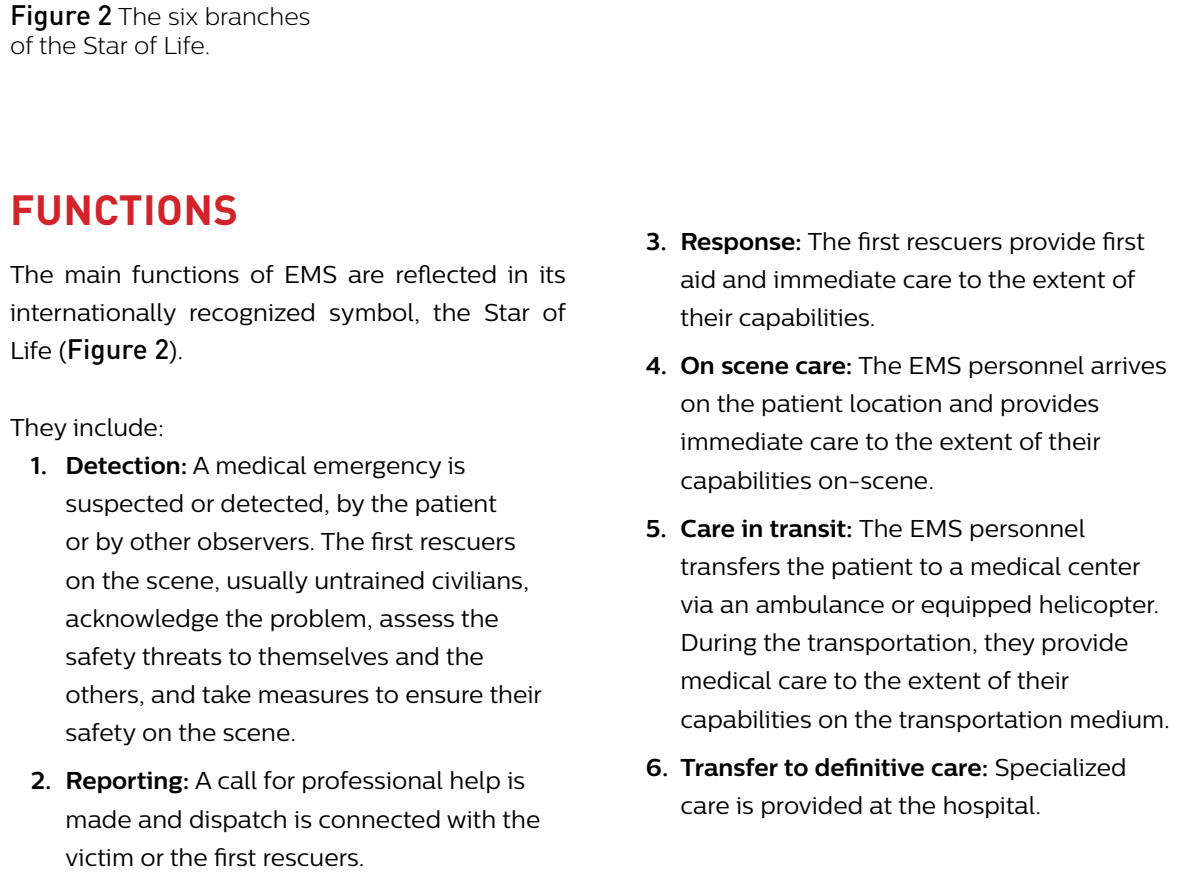

3. Response: The first rescuers provide first aid and immediate care to the extent of their capabilities.

4. On scene care: The EMS personnel arrives on the patient location and provides
immediate care to the extent of their capabilities on-scene.

5. Care in transit: The EMS personnel transfers the patient to a medical center via an ambulance or equipped helicopter During the transportation, they provide medical care to the extent of their 6. Transfer to definitive care: Specialized care is provided at the hospital. 


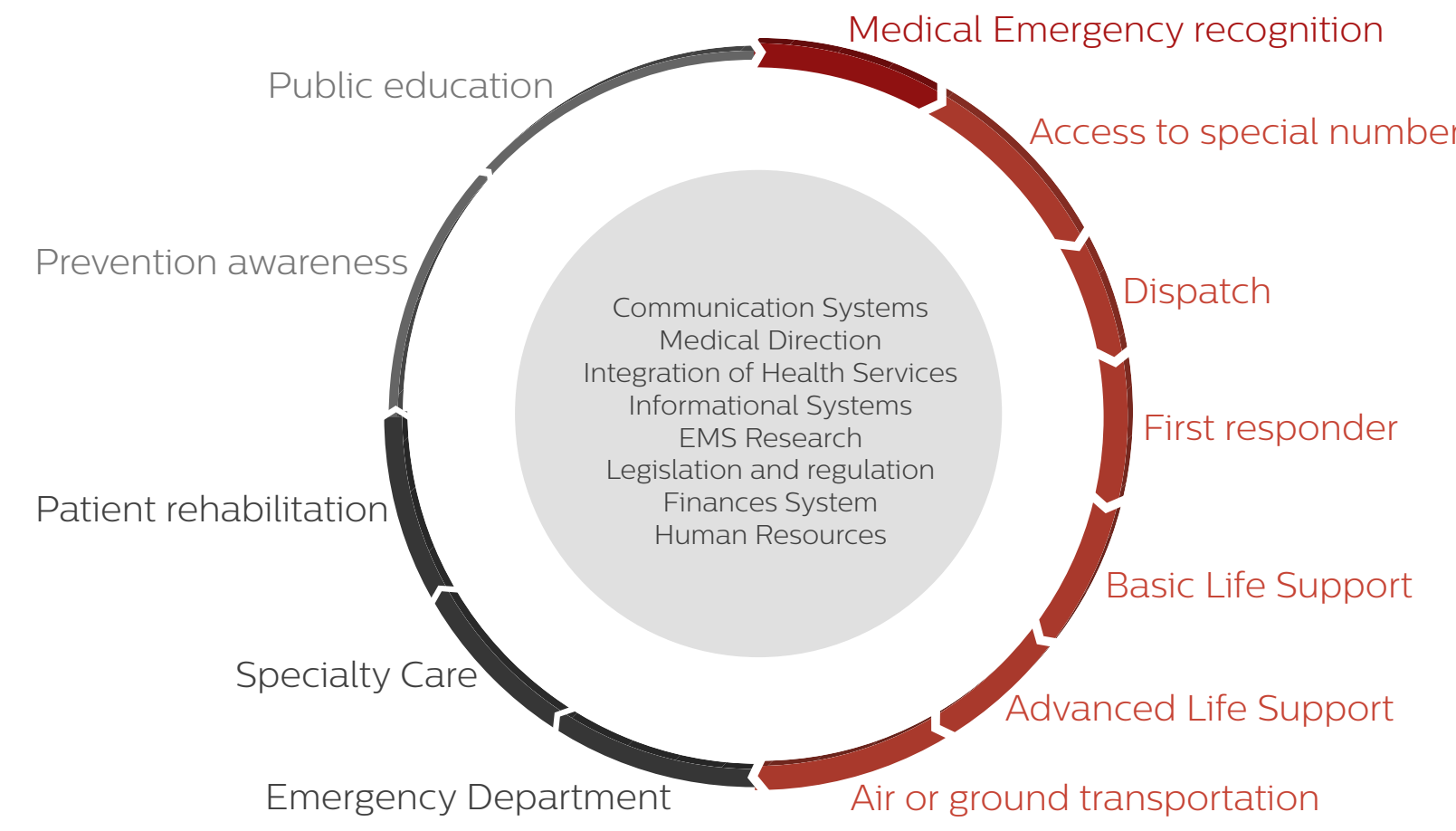

Figure 3 Protocols and

sub--systems connected to
EMS (adapted from NHTSA
2015).

\section{PROTOCOLS}

Even though EMSs organizational structures can vary significantly from country to country. common practices can be recognized above the

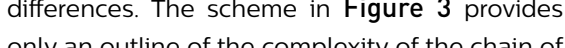
only an outtine of hie complexty of the chain of The outer circle represents

activated one after the other in res protocols medical emergency. In the center of the circle. are the sub-systems working 'behind the scene' to support the outer circle's elements. The red section represent the phases more closely pertaining emergency medical services.

\section{LEVELS OF CARE}

Emergency Meaical Services worldwide are provdedusing large spectrim a or menods

As a generalization, they can be categorized into (BIS) Advanced life Support Basic Life Suppor Transport (CCS).

A summary of the features of each care level is provided in Figure 4
WHO

\begin{tabular}{|c|c|c|c|}
\hline $\mathrm{WHO}$ & WHAT & WHAT NOT & HOW \\
\hline \multicolumn{4}{|c|}{ BASIC LIFE SUPPORT (BLS) } \\
\hline $\begin{array}{l}\text { Trained medical } \\
\text { personnel } \\
\text { Citizen volunteers } \\
\text { Often: Firefighters } \\
\text { and police }\end{array}$ & $\begin{array}{l}\text { Support the basic } \\
\text { functions of life- } \\
\text { threatened patients } \\
\text { until they can be } \\
\text { given full medical } \\
\text { care }\end{array}$ & $\begin{array}{l}\text { Drugs } \\
\text { administrations } \\
\text { Invasive procedures }\end{array}$ & $\begin{array}{l}\text { ABC (Airways, } \\
\text { Breathing, } \\
\text { Circulation) control } \\
\text { and support } \\
\text { CPR } \\
\text { (cardiopulmonary } \\
\text { resuscitation) } \\
\text { Artificial respiration } \\
\text { Bleeding Control } \\
\text { Use of AED } \\
\text { (Automated External } \\
\text { Defibrillator) }\end{array}$ \\
\hline \multicolumn{4}{|c|}{ ADVANCED LIFE SUPPORT (ALS) } \\
\hline $\begin{array}{l}\text { Physicians } \\
\text { Nurses } \\
\text { Often: Medical } \\
\text { Technicians }\end{array}$ & $\begin{array}{l}\text { Support the basic } \\
\text { functions of life- } \\
\text { threatened patients } \\
\text { including invasive } \\
\text { procedures } \\
\text { and specialized } \\
\text { equipment }\end{array}$ & $\begin{array}{l}\text { Diagnosis } \\
\text { Surgery } \\
\text { Use of ICU } \\
\text { equipment }\end{array}$ & $\begin{array}{l}\text { All of the BLS } \\
\text { protocols } \\
\text { Tracheal intubation } \\
\text { Rapid sequence } \\
\text { intubation } \\
\text { Cardiac monitoring } \\
\text { Cardiac defibrillation } \\
\text { Transcutaneous } \\
\text { pacing } \\
\text { Intravenous } \\
\text { cannulation } \\
\text { - Intraosseous } \\
\text { infusion } \\
\text { Surgical } \\
\text { cricothyrotomy } \\
\text { Needle } \\
\text { cricothyrotomy } \\
\text { Medication } \\
\text { administration } \\
\text { Cardiac Life Support }\end{array}$ \\
\hline \multicolumn{4}{|c|}{ CRITICAL CARE TRANSPORT (CCT) } \\
\hline $\begin{array}{l}\text { Trained Nurses } \\
\text { Often: Respiratory } \\
\text { Therapist (RT). }\end{array}$ & $\begin{array}{l}\text { Transport critically } \\
\text { ill patients from one } \\
\text { medical facility to } \\
\text { another ensuring } \\
\text { comfort and safety. }\end{array}$ & $\begin{array}{l}\text { Diagnosis } \\
\text { Surgery }\end{array}$ & $\begin{array}{l}\text { All of the ALS } \\
\text { protocols } \\
\text { Use of ICU } \\
\text { equipment (e.g. } \\
\text { Automatic Transport } \\
\text { Ventilators (ATV) for } \\
\text { ventilator dependent } \\
\text { patients). }\end{array}$ \\
\hline
\end{tabular}




\section{EMS in the world}

context introduction

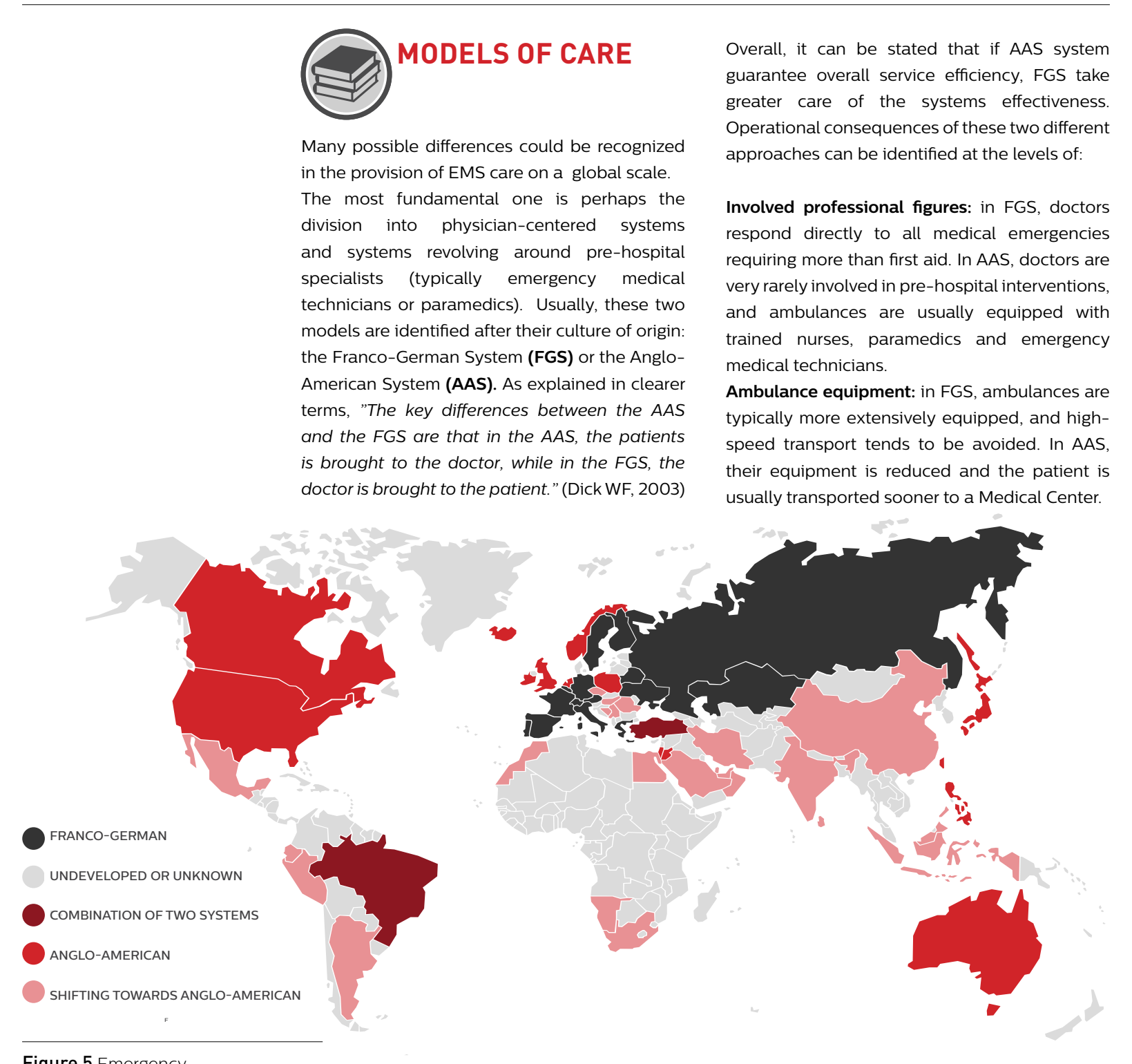

Figure 5 Emergency
Medical Systems mod
FRANCO-GERMAN SYSTEM

scoop and run

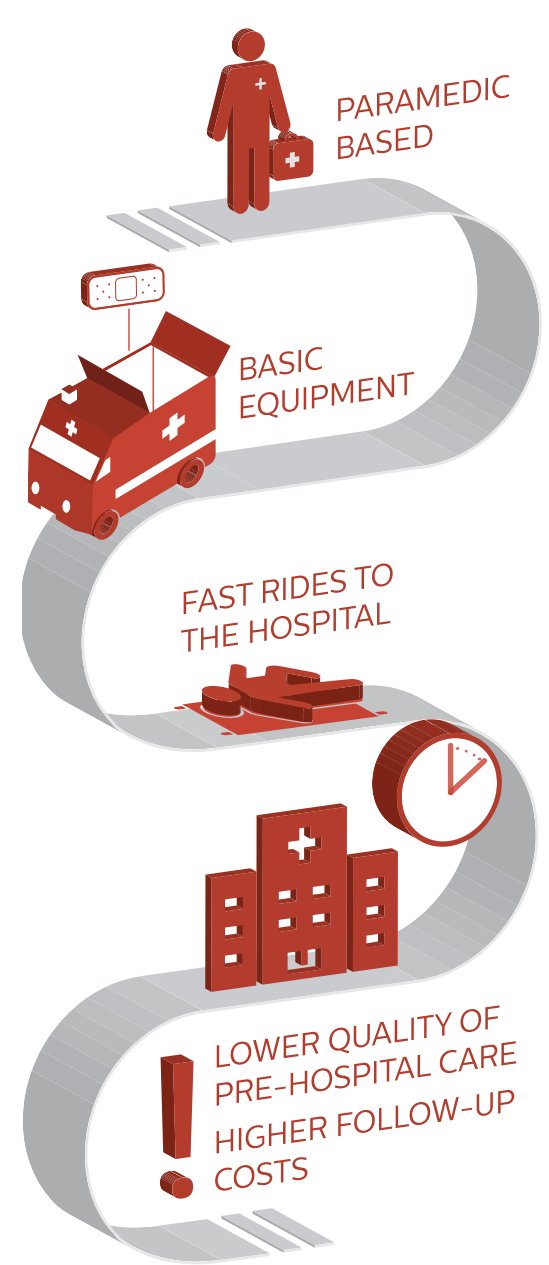

Clinical governance: In most FGSS, pre-hospita specialists are only authorzed to provide care in presence of a physician. In many parts of the world (e.g. France, Belglum, Luxembourg, italy Span, Barlical Su cavision on a permanent Cons

Cospital staff is given decisions. This authority is built on the basis of offline protocols and, only if needed, by voice consultations with online physicians. Both the protocols and the permissions given to the pre-hospital staff is provided by one or more physicians charged to establish the emergency cal directive for a specific region. stay and stabilize

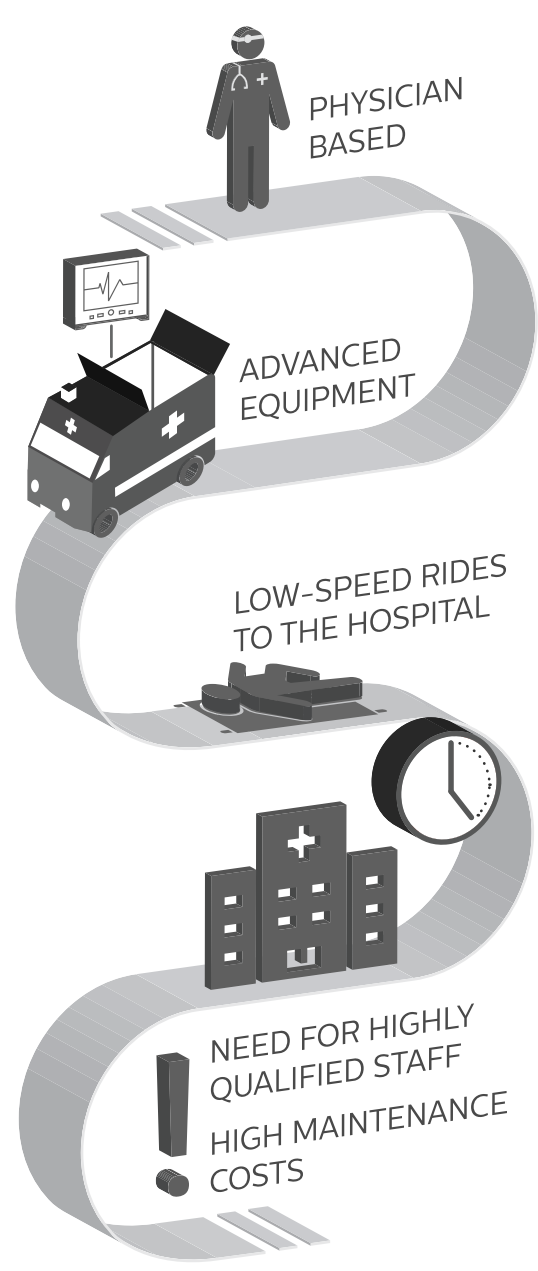

From this point on, the Anglo-American model will be addressed as the project target context, manty in consideration of large application Figure 5) Furthermobe, scale (as shown in basis of this poject will be condect in close collaboration with hospitals in the Netherlands the Dutch model will be analyzed and used as a

Nevertheless, the peculiarities of the different models will be kept in consideration during later project stages, and the possible validity of the generated solution in Franco-German systems 


\section{EMS in the Netherlands}

\author{
Mapping the stakeholders
}

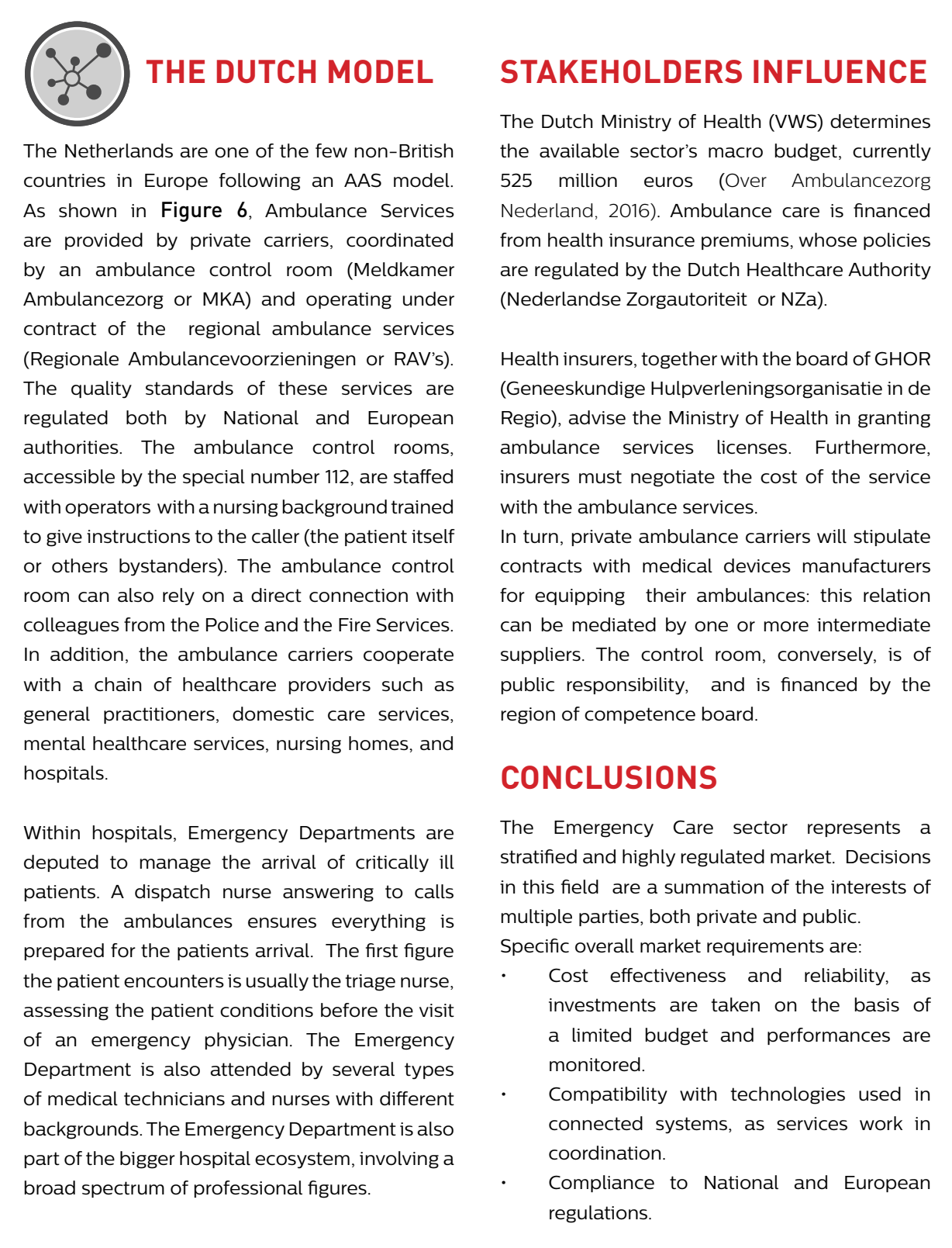

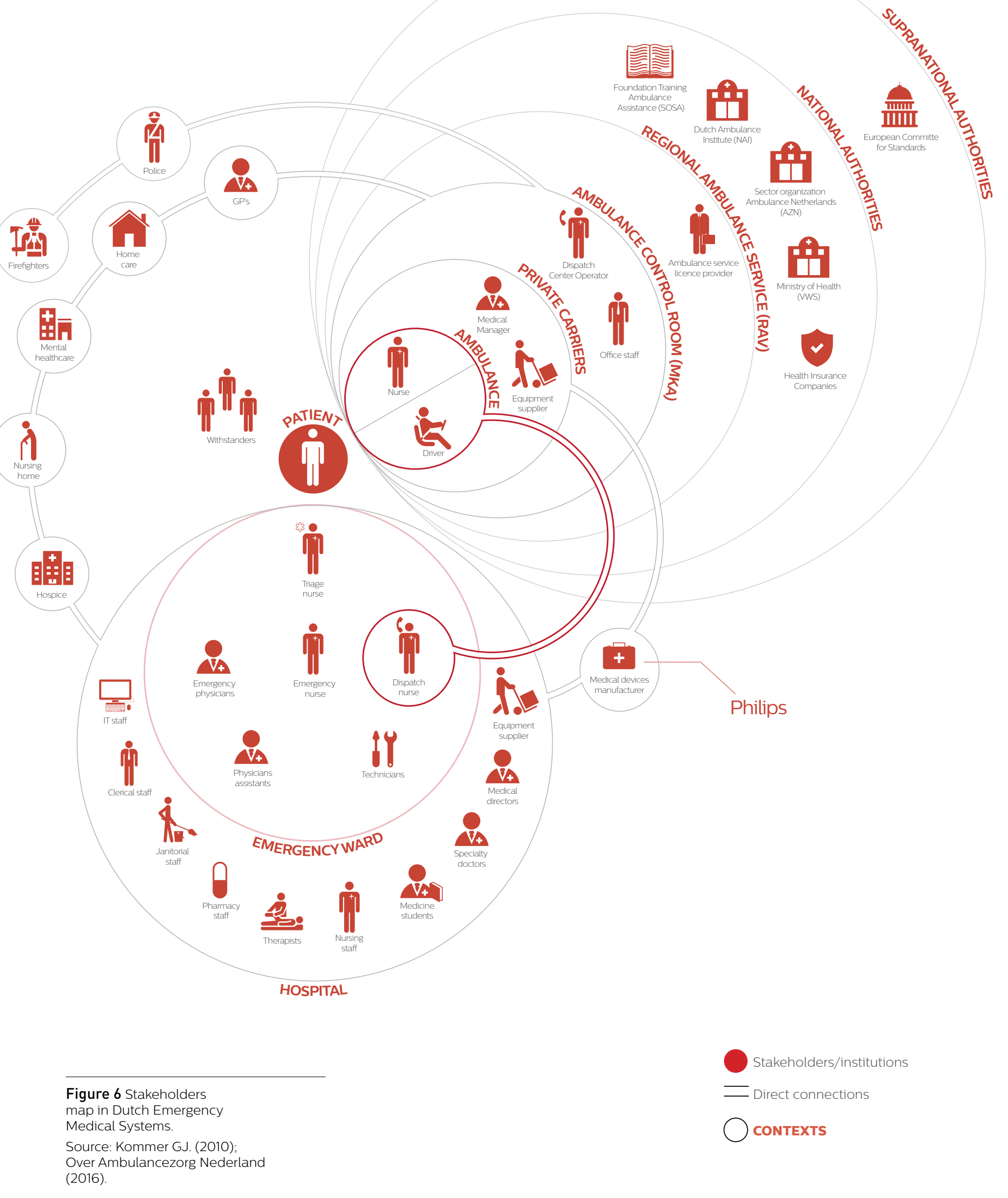




\section{Ambulance rescuing}

Not just driving patients

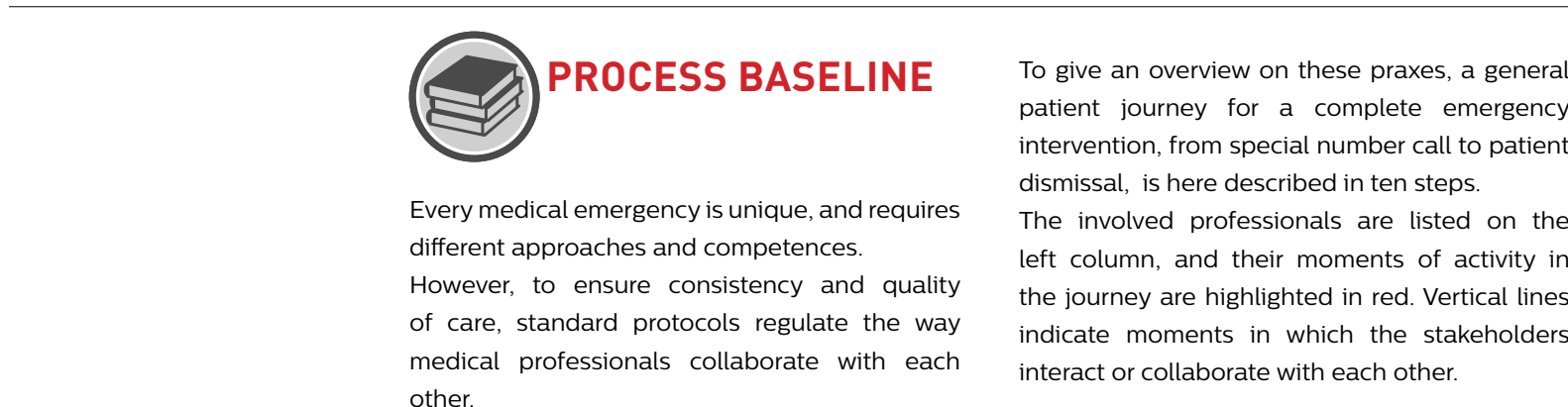

PROCESS FEATURES

Out of the outlined journey, some considerations can be taken regarding characteristics and peculiarities of the overall process tion is that all of the complex sets of protocols, taking place astuation of time pressure More in depth is to be noticed how each and every stage s chained and every time delayin one step has a cascade effect on the whole process.

To this end, the transitions between one stage and the other (steps $2,4,5,6,8$ ) appear as senstive moments whose flidity is " crucial to ensure optinal process speed . workers
This operational framework is, in traditional A final important consideration regarding EMS lierature (Arman et al, 1999), often - the fourney is that most of the interactions atculated on the three perspectives of:

Mutual awareness (every operator is aware of the role and competences of

(the segation assessment medical emergency is assessed in collaboration between several operators)

Co-ordinated use of technology (operators can rely on a common

Falures under any of these perspectives a en incidents, in every dymamic complex system relying on the efforts of multiple ndividuals.

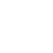
(n) constituted by exchanges of information (steps $1,2,4,5,6,7,9)$ ). Information, in fact, appears to be the for activaling and emergency management process.

To this end, is it beneficial to denote how effective and precise communication plays a delicate but pivotal role in ensuring patient's safety throughout the rescue process. According to Shelton and Sinclar (Shelton et al, 2016), communication fallues stil consthute the man cause for adverse events in healthcare.

To provide a more complete overview on the topic the next chapter has been reserve to the description of how information is generated and propagated within EMSs.

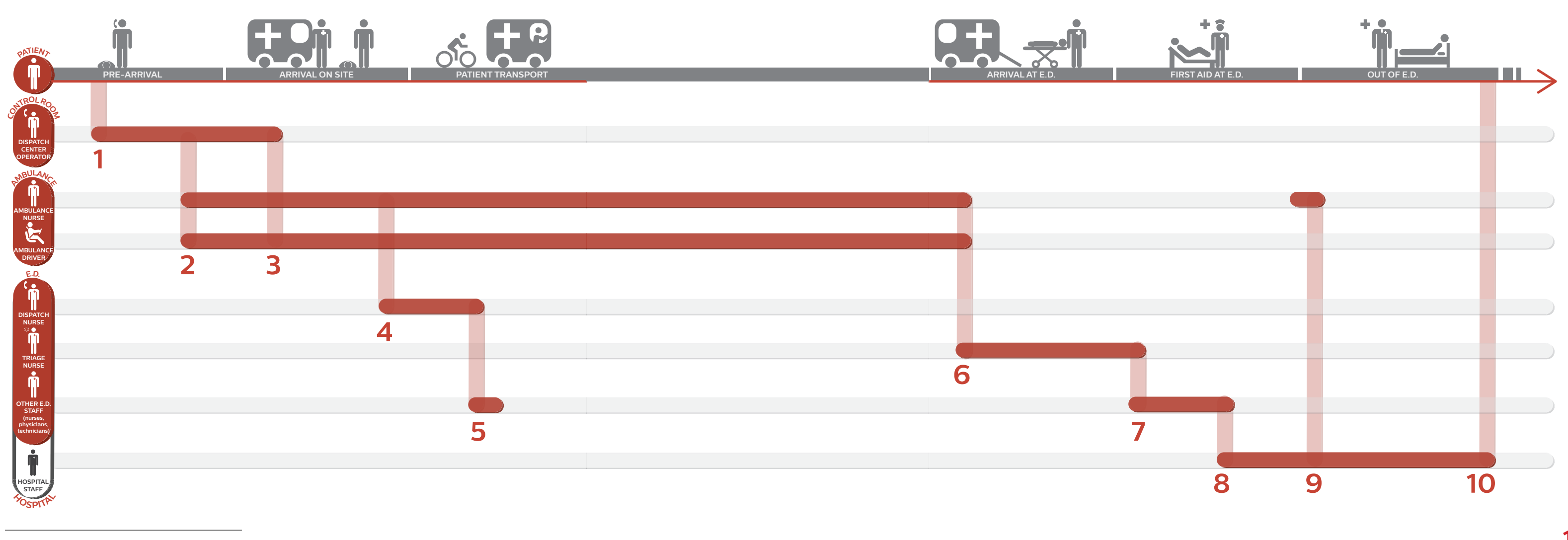

A medical emergency is reported to structions.

2 If necessary, the operator calls emergency details and location. 3 The ambulance crew assesses
the case, provides first aid and, if 4 The ambulance crew informs the dispatch nurse on the arrival and gives

5 The disper

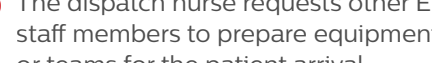

6 The ambulance nurse hands the

7 The triage nurse labels
with a priority code.

8 The patient's acute situation is moved to another department.

9 The ambulance nurse fills a report
with the details of the case and hands

10 The patient is given full medica 


\section{Communication in EMS}

The 'Chinese whispers'

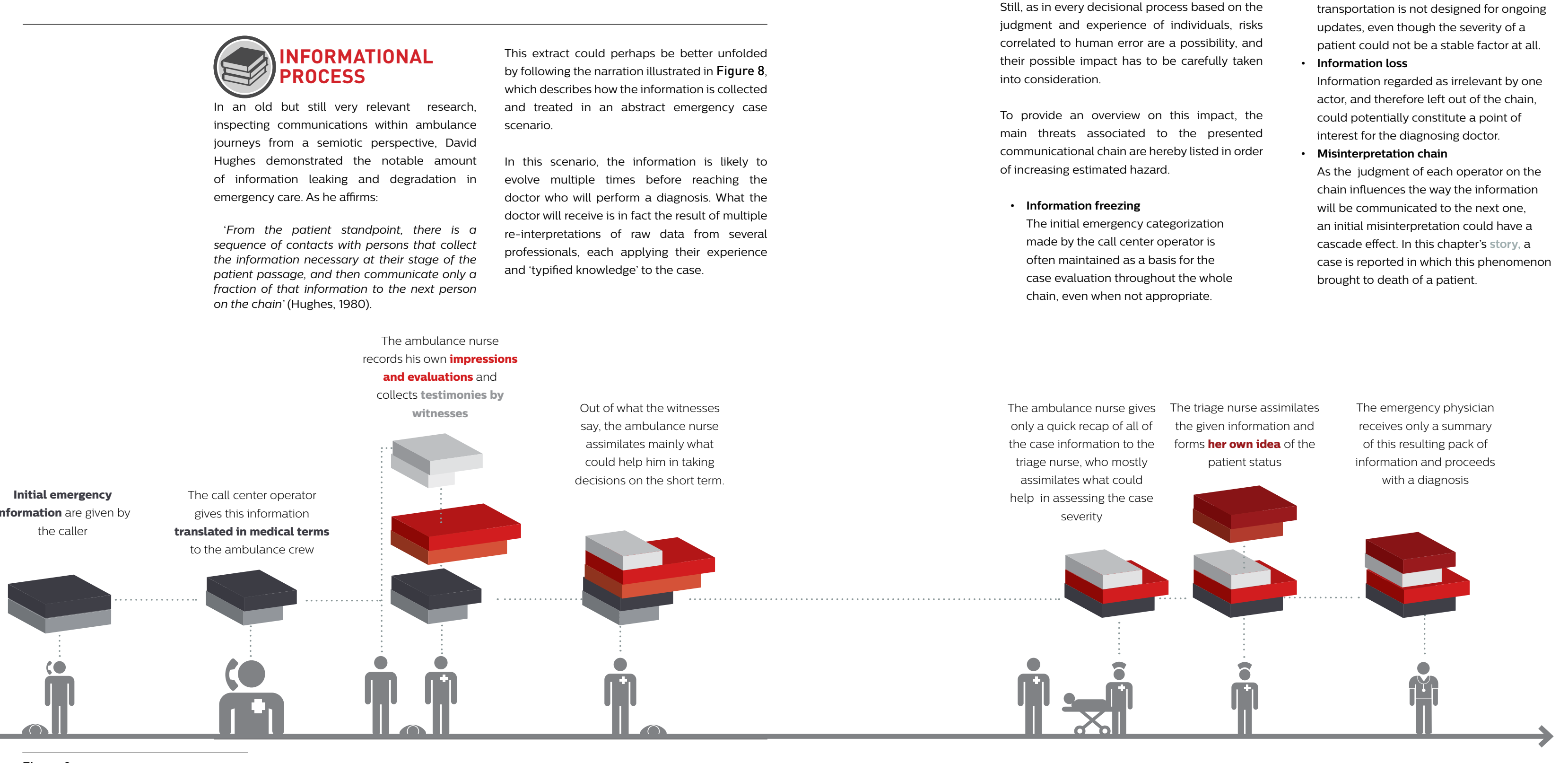

Figure 8 Data set evolution in
the first stages of emergency
PROCESS EFFECTIVENESS

The described multi-step passage of information, as a communicational method in EMS, presents undoubled advantages. In fact . constrains are extreme and fast decision has a fe-saving value.

till, as in every decisional process based on the

provide an overview on this impact, the owledge' to the case. main threats associated to the presented scenario, as no later inspection or hearing
Also, the information collected by the ever be collected about the emergency oftestimonies is performed. In the moment regarding the scene is said to be 'frozen' (Hughes, 1980) Finally the communication from the ambulance to the hospital during levant by on
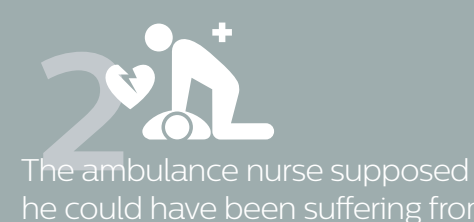
a heart attack

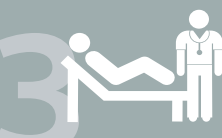

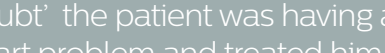
cart probiem and treated him an

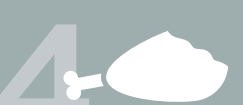




\section{Teamwork in EMS}

\begin{tabular}{|c|c|}
\hline $\begin{array}{l}\text { (i) INFORMATIONAL } \\
\text { DYNAMICS }\end{array}$ & $\begin{array}{l}\text { This model lies on the principle of analyzing } \\
\text { cognition as if it was not a phenomenon } \\
\text { 'bounded to the individual brain' (Resnick, 1991), } \\
\text { but rather a team activity, in which different }\end{array}$ \\
\hline $\begin{array}{l}\text { As we have seen, a typical EMS informational } \\
\text { chain encapsulates acts of conscious mental } \\
\text { activities, as the way individuals in the chain } \\
\text { think, understand, learn and remember has } \\
\text { certain effects on the information itself. In } \\
\text { cognitive ergonomics literature, a framework } \\
\text { often used to describe these type of systems is } \\
\text { the model of distributed cognition. }\end{array}$ & $\begin{array}{l}\text { elements (minds and artifacts) contribute to an } \\
\text { overall cognitive process. } \\
\text { Evaluating systems in terms of distributed } \\
\text { cognition or individual cognition could differ } \\
\text { drastically: for instance, 'an individual optimal } \\
\text { performance may be suboptimal, in relation } \\
\text { to the overall goal shared by the whole team' } \\
\text { (Artman, 1998). }\end{array}$ \\
\hline
\end{tabular}

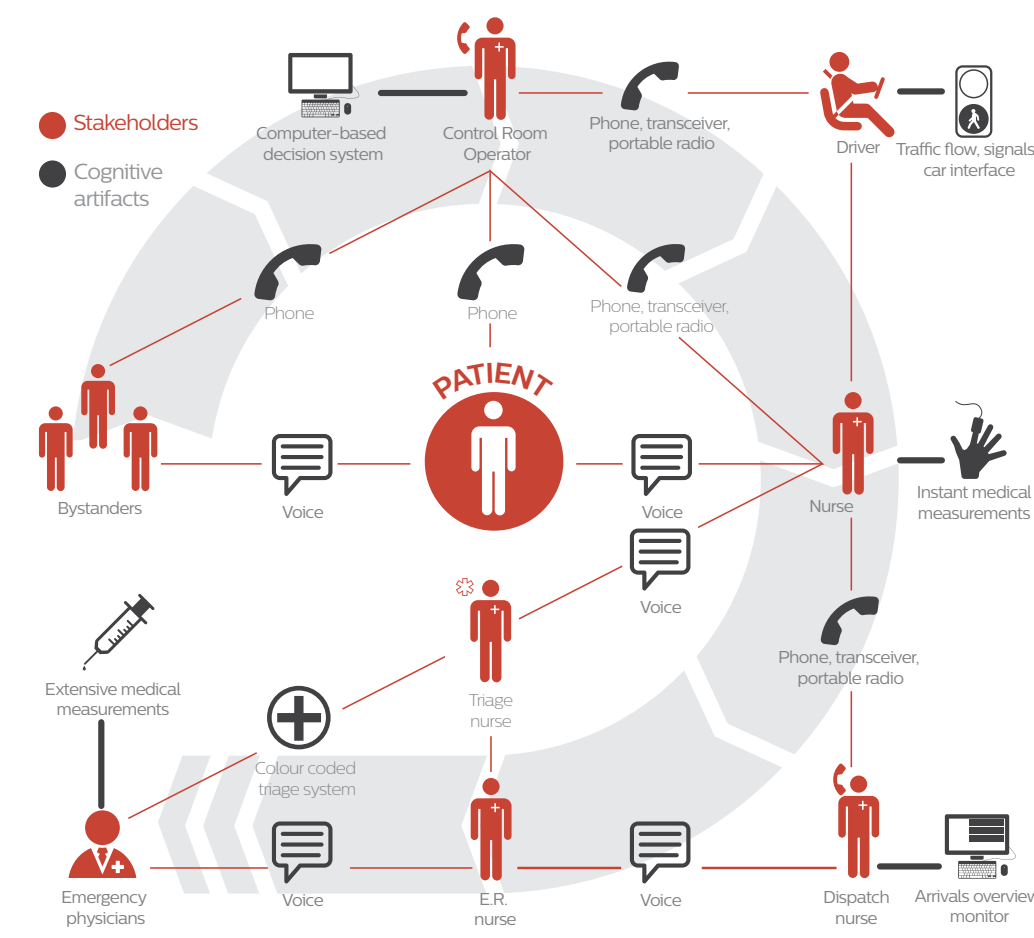

Figure 9 Cognitive channels in
ambulance nursing
Also, in distributed cognition terms, artifacts are not only examined for the efficiency they relation to their role as coordination tools.

Due to its appropriateness to describe and evaluate Emergency Medical System this context (Furniss, 2006). An overview of the collaborations among stakeholders contributing to distributed cognition in ambulance nursin including the main cognitive artifacts used, is offered in Figure 9

As it can be seen, an inefficiency in any part of the network would constitute a direct damage to

Reaching optimal awareness on the emergency case, and

While some artifacts are explicitly designed for coordinating the distributed team (e.g. phones) other constitute a cognitive source that needs to be consciously assimilated and transformed into meaning by team members (e.g. instant measuring instruments information has to be

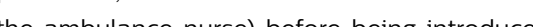
the cognitive network.

In this sense, shared cognition principles would suggest that enabling an automatic availability of these information to other team members would be a way to improve the coordination of the overall team. The potental value of such an intervention wil be examined further dumn desien opporturity INFORMATIONAL CHANNELS fter the information content and the dynamics have been described, a last perspective under which com dsente a last

Observing Figure 9 again, we can see how the exchanges of data in the system happen under a number of different forms, both verbal and nonverbal: phone calls, face-to-face, written tex color coding are only some of the examples
Notably, some of these forms are volatile and leave no trace, while others allow for long-term storage. This diversity is considered to result from an ongoing change in nursing common systems (Wison, 5 20 paper-based to digital practical neds s in par), and from oventing Under this perspective there is little surprise that the two main communication channels active in ambulance nursing are the visual and the auditory one.

In a recent study (Zaborowski, 2015) investigating how ambulance nurses prefer to receive information, $86 \%$ of the interviewed selected the auditory channel as their favorite medium, while same set of particisats freauent problems connected to the usage of the auditory channel to communicate in fact $72 \%$ of the overall group identified noise as a frequent cause of disturbance in long-distance voice communications. This might explain why, when asked which communicational systems were in their opinion the fastest and most unambiguous ones, the proportions changed. where $20 \%$ op ped forvsul

All in all, these results seem to outline the presence of a need for faster more unambignous and undisturbed channel than the ones currently available. This possible problem area will be kept
in consideration for further validation with users.

\section{CONCLUSIONS}

Mapping processes connected to ambulance rescuing brought to an acknowledgment of the crucial importance of communication.

Therefore, the features of communication in ambulance rescuing were analyzed under
several aspects. The main insight from these analyses are summarized as

The communications between

stakeholders in EMS happen under many

but they always rely on a same codifis,

language 


\section{Trends and directions}

\begin{tabular}{|c|c|}
\hline & \\
\hline (5) ) & SOCIETY AND \\
\hline $\begin{array}{l}\text { (E) component of planning a successful } \\
\text { innovation strategy. }\end{array}$ & $\begin{array}{l}\text { For what concerns the Netherlands, a shifting of } \\
\text { responsibilities in the medical field is expected } \\
\text { to happen on many layers. On the institutional }\end{array}$ \\
\hline xpected trends from the economical, & level, a rearrangement of hospital systems is \\
\hline ical and technological domain have & going to aim at centralizing clinical specialties in \\
\hline$d$ in Figure 10 that could have a & \\
\hline & On the professional level, roles are mutating: \\
\hline & $\begin{array}{l}\text { general practitioners will increasingly be } \\
\text { assigned tasks previously carried out in hospital }\end{array}$ \\
\hline TECHNOLOGY & environments. Also, policemen and firefighters \\
\hline Big innovations expected to stream the future & $\begin{array}{c}\text { crews will be more and more equipped with } \\
\text { automated external defibrillators }\end{array}$ \\
\hline $\begin{array}{l}\text { tech (such as digital health, data } \\
\text { wearable medical sensors and in }\end{array}$ & $\begin{array}{l}\text { and trained to intervene in certain medical } \\
\text { emergency situations. }\end{array}$ \\
\hline $\begin{array}{l}\text { dual health monitoring) all contain } \\
\text { ig value in the field of Emergency }\end{array}$ & On the organizational level, the increasing \\
\hline & $\begin{array}{l}\text { digitalization of protocols and communications } \\
\text { will expand into new areas, while the solutions }\end{array}$ \\
\hline & $\begin{array}{l}\text { that are already implemented will evolve and } \\
\text { start to merge with each other. }\end{array}$ \\
\hline & \\
\hline field, could find several possible applications & CONCLUSIONS \\
\hline $\begin{array}{l}\text { of monitoring remotely the operational status } \\
\text { of emergency medical equipment to prevent } \\
\text { unexpected failures. }\end{array}$ & $\begin{array}{l}\text { It is expectable to observe, in the near future, } \\
\text { a shift towards digitalization for many aspects } \\
\text { of Emergency Care: medical data collection, }\end{array}$ \\
\hline ECONOMY & $\begin{array}{l}\text { delivery and analysis, equipments performances } \\
\text { monitoring, health management. Moreover, }\end{array}$ \\
\hline the economical side, several challenge & $\begin{array}{l}\text { most of the upcoming interventions in } \\
\text { Emergency Care are likely to be primarily aimed }\end{array}$ \\
\hline $\begin{array}{l}\text { ted to innovators in the coming years, } \\
\text { growing labor and equipments costs }\end{array}$ & at reducing operations time, budget and medical \\
\hline & staff labor, rather than improving service quality. \\
\hline & Therefore, future solutions for this context will \\
\hline & 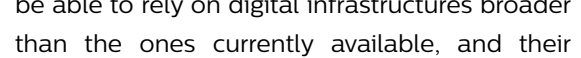 \\
\hline $\begin{array}{l}\text { migrating: risks costs, for instance, are } \\
\text { coming less and less a poblic concern and }\end{array}$ & adoption will be mainly determined by their \\
\hline & \\
\hline
\end{tabular}

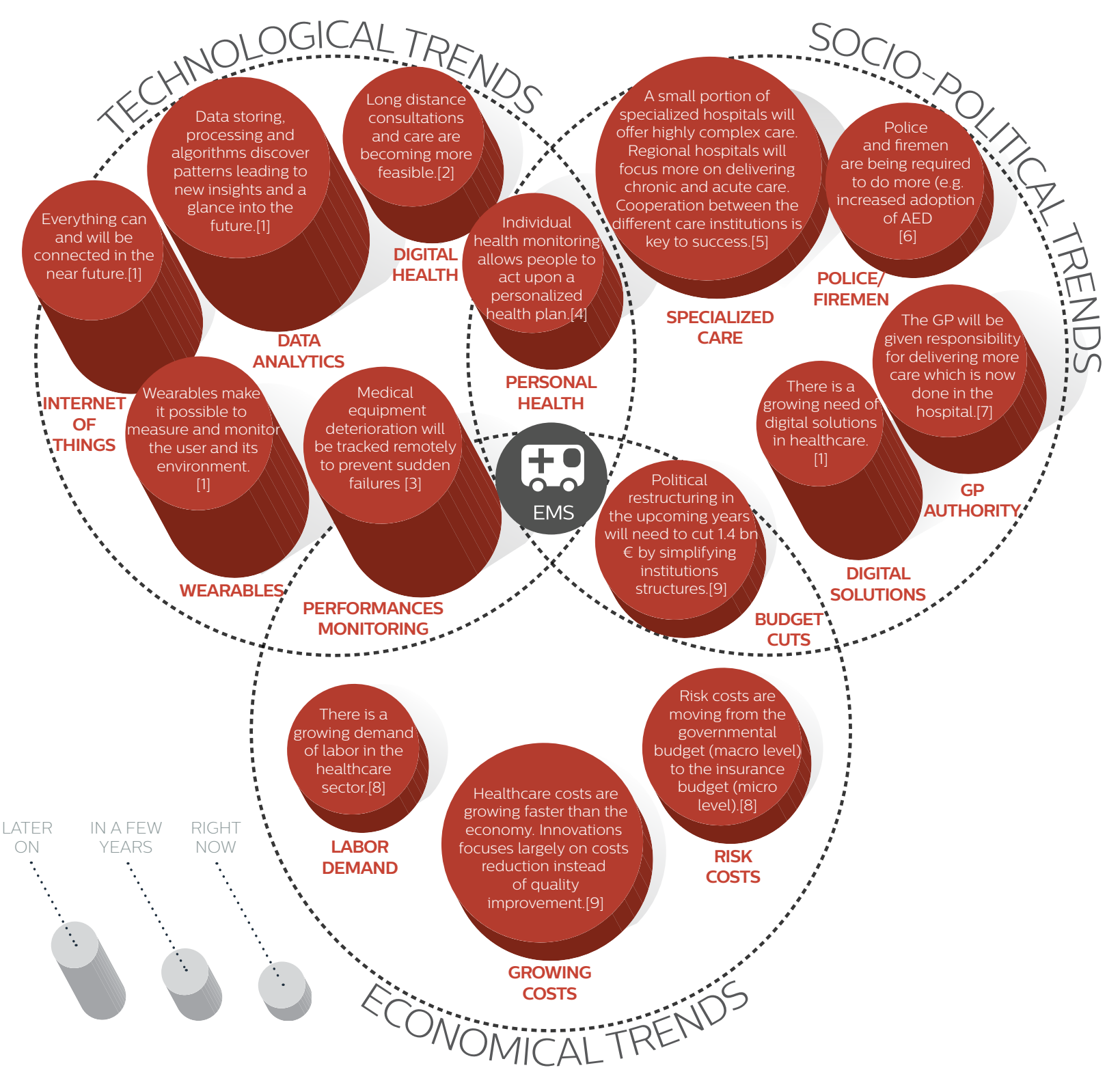

\section{Figure 10 Upcoming trends
in Dutch Emergency Medical}

in Dutch Emergency
Systems. Sources:

Brookman, D. (2014). Introducing experience domains. Connecting the Dots, (1)

3. Mwanza, B.G. and C.M Mbohwa (2015). "An Assessment of the Effectiveness of Equipment Maintenance Practices in Public Hospitals." Procedia
Manufacturing 4: 307-314.

4. Phelps, M. (2013, November 26). 10 megatrends shaping healthcare. Retrieved September 23, 2015

5. Dantuma, E. (2015, March 15). Verbeteren door te verbinden. Retrieved September 23,2015

7. Gevolgen milijonennnota 2016 vor zorg \& 8 welzijin. (nod.). Retrieved September 23,2015 ,

9. Rabobank ciffers en trends: Gezondheidszorg (n.d.). Retrieved September 23, 2015 


\section{Preliminary}

\section{observation}

Approaching the field

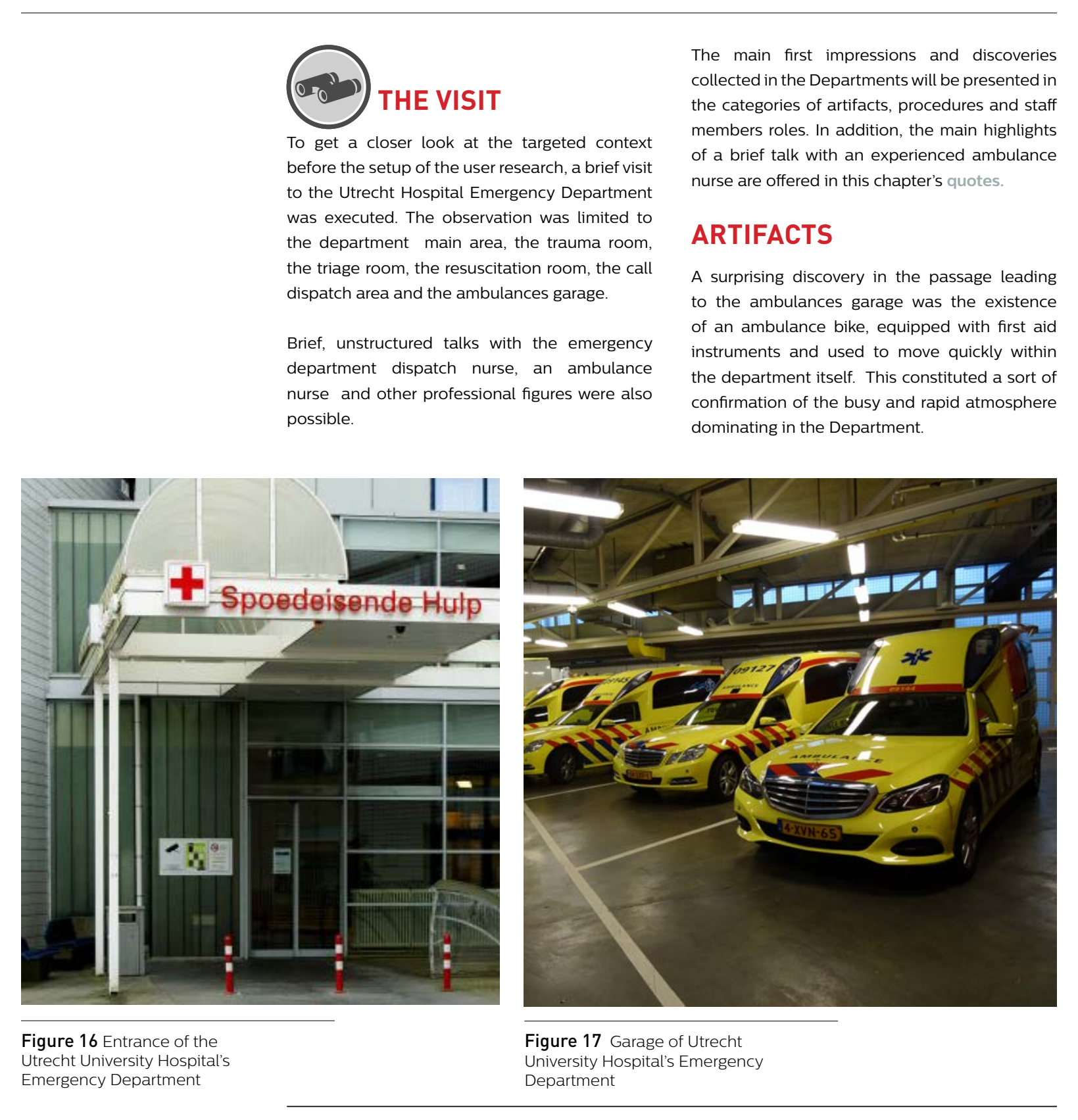

A less evident but as interesting discovery was ROLES

constituted by the number of paper-based decision alas avallable in afiserent a Among others, the following were spoted Emergency department timeout checklist patients.

Folders of laminated paper sheets,

政

equipment in pediatric cases (tables a size- suggested device).

A paper template hanging in the trauma room, filled in by the dispatch nurse, containing basic information about the upcoming case for when the trauma team is preparing. The data provided in mechanism of iniury blood type and a (n) All over the Department booklets of approved protocols and decisional flowcharts for different medical emergencies were available.

This abundance of hard copy material was explained with the urge for having the needed Information immediately avalable in case of served as a way to diffuse potentially crucial information in the Department environment to be as close as possible to the operators workin on the case if needed.

PROCEDURES

Astriking initial impression about the Departmen egarded the dynamism and variety of workin

As testified by the dispatch nurse, shifts of doctors are frequent, and the main continuity is given by the nurses. A consequence of this is that emergency teams could be formed of people who have never met each other before.

The same dispatch nurse, when asked abou the knds or information wanted when receiving data such as name and ase in order to stat the internal procedures The availability of this piece of data interestingly, was also mentioned as an issue by the ambulance nurse (see guntes)

\section{The}

A talk with the dispatch nurse pointed out a number of unforeseen tasks falling under her
responsibilities; for instance, she mentioned how many calls are coming from families and friends 政 or emergency control rooms.

Regarding the psychological attitude toward her works, she stressed out the difficulty of not letting empathy arise when receiving 'bad news' In her opinion, operators feel the impulse to react emotionally to accidents as everyone else. This will be kept in consideration, and investigated further in the user research.

Other discoveries about roles and responsibilities within the Emergency Department concern: The ambulance driver, being occasionally deputed to manage the call to the hospital The central role of triage nurses, their usually extensive backsround (often the everyone else in the Department)

CONCLUSIONS

The visit constituted an essential preparation for the user research. Topics suggested after for further exploration are:

(E) " as the ambunce bike might subest the need for increased mobility. Inspecting the frequency and modality of use of the paper checklists, reminders information sheets

Observe consequent shifts paying attention at teamwork aspects (e. are there any problems in finding - Drofessionals?)

Emergency Department workers

Map the main roles and responsibilities of each professional role

\section{quotes}

0

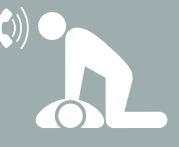

$+0$ 


\title{
Preliminary research
}

\author{
methods
}

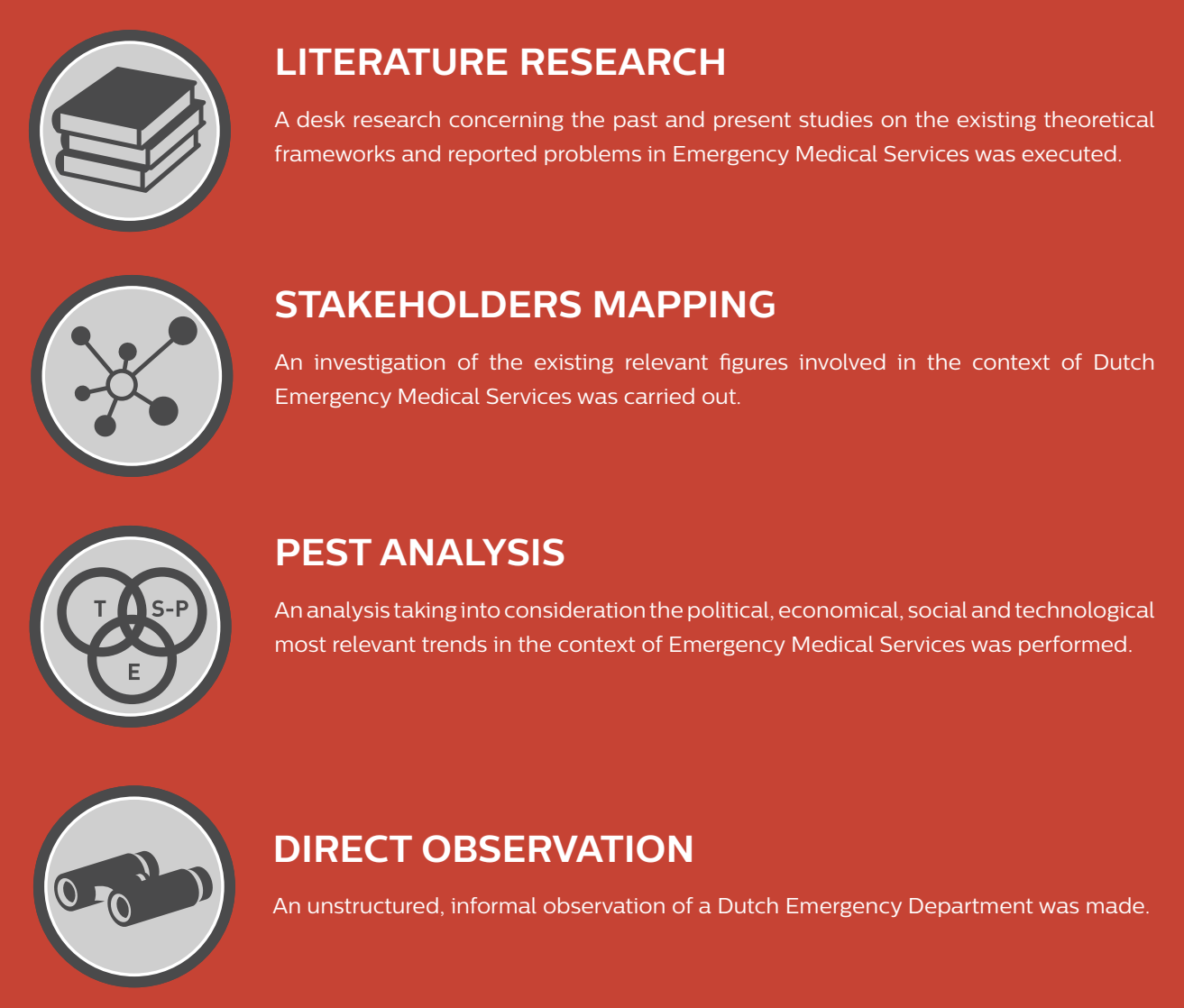

\section{Preliminary research} theoretical framework

DISTRIBUTED COGNITION

(1i) The framework of Distributed Cognition, defined in cognitive science as an approach
to cognitive analysis taking into consideration the totality of indivduals and

communicational artifiacts as a unitit was presesented and adopted for describing the
Emergency Medical Services system. 


\section{Research set-up}

\author{
Approach and framework
}

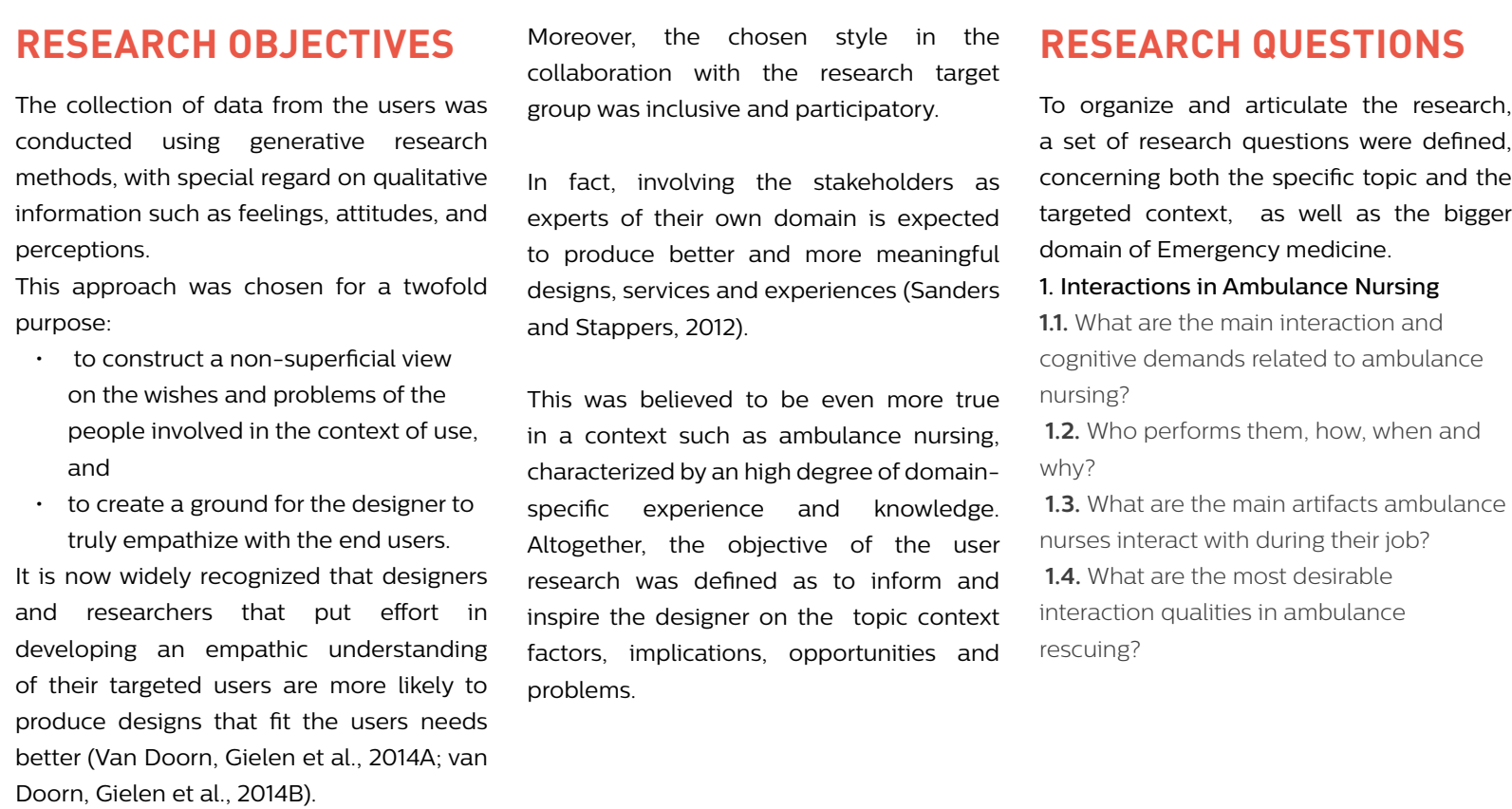
produce better and more meaninful domain of Emergency medicine. designs, services and experiences (Sanders 1 . Interactions in Ambulance Nursing and Stappers, 2012). This was believed to be even more true nursing? 12. Who performs them how when and

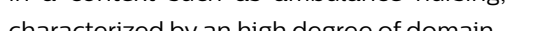
mecific experience and knowledge. 1.3. What are the main artifacts ambulance Atogether, the objective of the user nurses interact with during their iob? research was defined as to inform and 1.4. What are the most desirable inspire the designer on the topic context interaction qualities in ambulance factors, imm
problems.

interaction
rescuing?

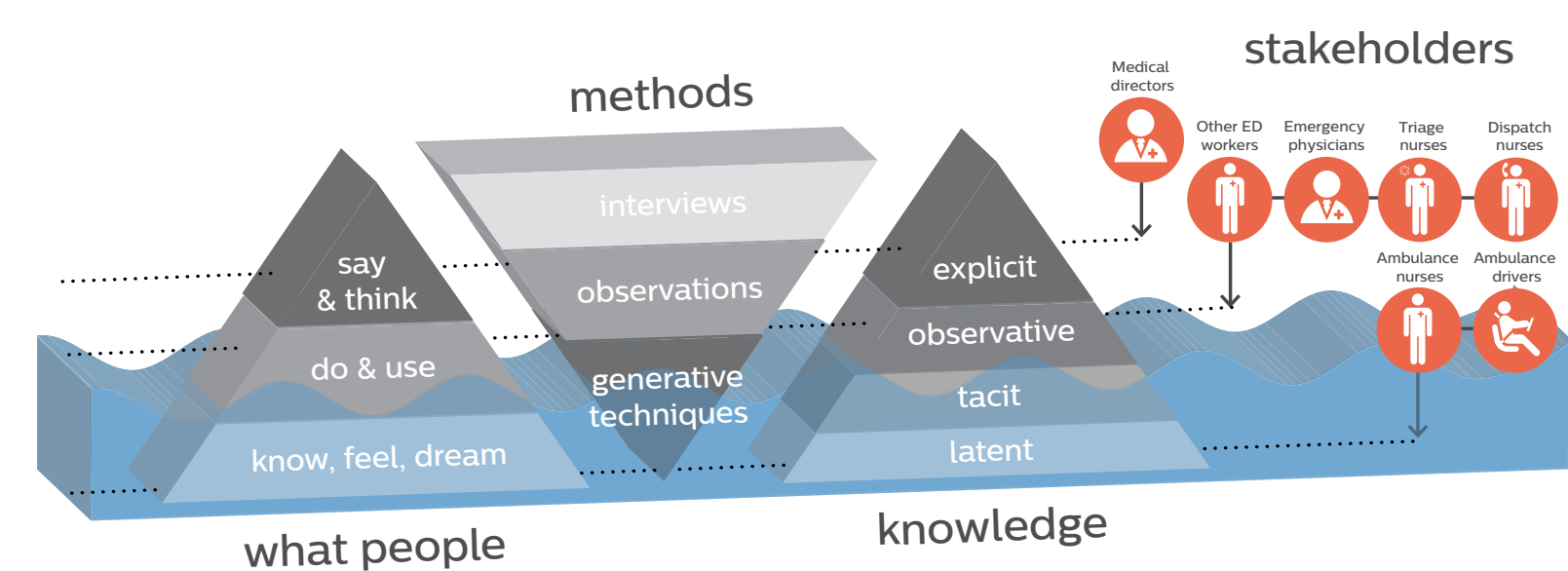

Figure 18 Research depth for
context stakeholders
2. Ambulance nursing context 21. What are the main tasks and activities performed by amber
time do they take?

. What are the main parties ambulance

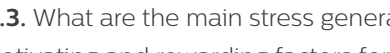
ambulance staff?

4 . What's on the top of ambulance mind while performing a rescue? 2.5. What does ambulance staff think vould make their job easier?

3. Emergency Medicine domain

3.1 What are the most improvable areas experts?

RESEARCH DEPTH

methods

upon the corpus of resers and Stappers, 2012). and resources, a deliberate selection was main focus of the research was maintain on the ambulance staff, identified mbulance nurse and driver. available, a classification was introduced in techniques revealing what people 'say and think, what people do and use and what

As the methods for accessing deeper Asvels of knowledge coincide with the more challenging ones in terms of time made on what types of stakeholders to analyze more in depth (see Figure 18). The

Other stakeholders, such as the Emergency Out of eight hospitals contacted to Department staff, were included in the gain access to Emergency Department research main scope but excluded from workers, only three ever reacted: two in-depth generative research methods. of these reactions brought to an actual Finally, stakeholders involved in decision collaboration, while the third response makng but far from the product-semice anved too late to be included in the on specific subjects.

RESEARCH BREADTH

Out of the twelve organizations contacted to get access to Ambulance Services employees, only five reacted Out of As stated in the preliminary research this five, two organization decided not chapter, the Anglo-American model of to participate in the research, while the emergency care consthutes the project remaining three demonstrated interest in mann targeted context. Consequenty, the topic and confirmed heir willingness in countries following this system (see Figure 5 for an overview) was maintained

In particular, all of the research off-line the Netherlands, and the results obtained the seeking of participants in the medical werentegrated wh on-line questionnares field has been drafted for future reference directed to USA and Canadian ambulance in Appendix 1.

PARTICIPANT

RECRUITMENT

attempted using both formal and informal media, such as e-mail phone call, socral media (racebook,

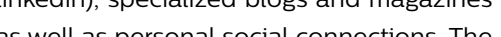
compant
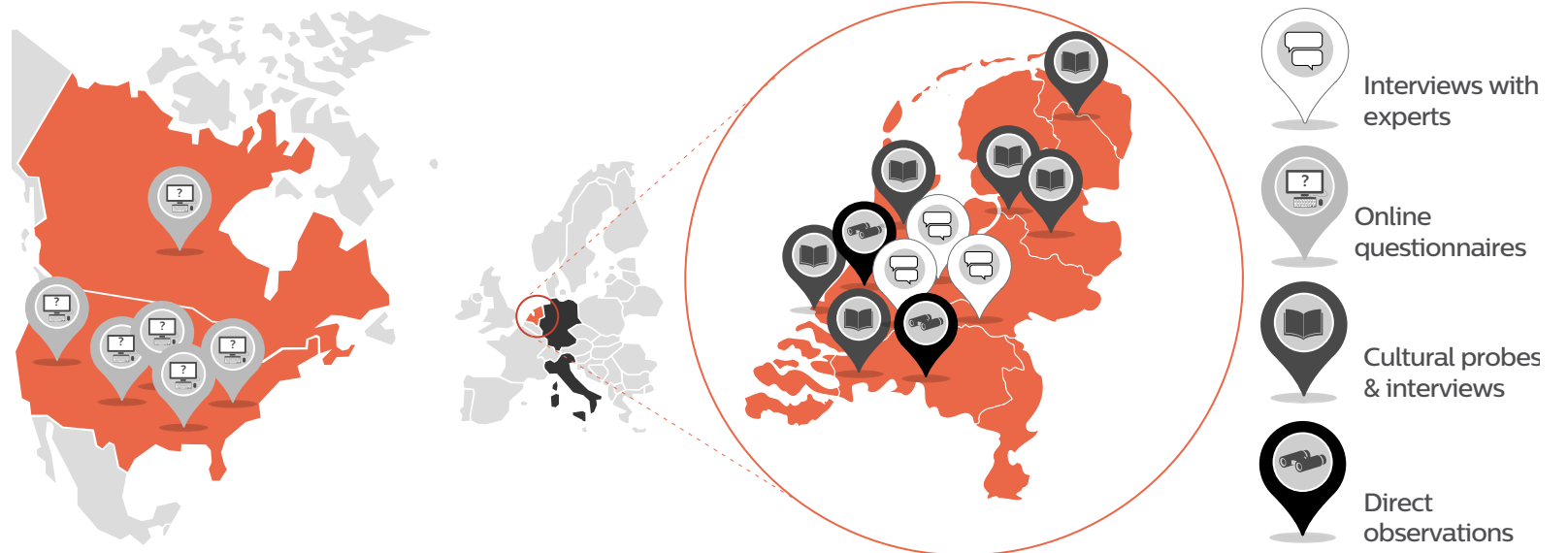


\section{Research methodology}
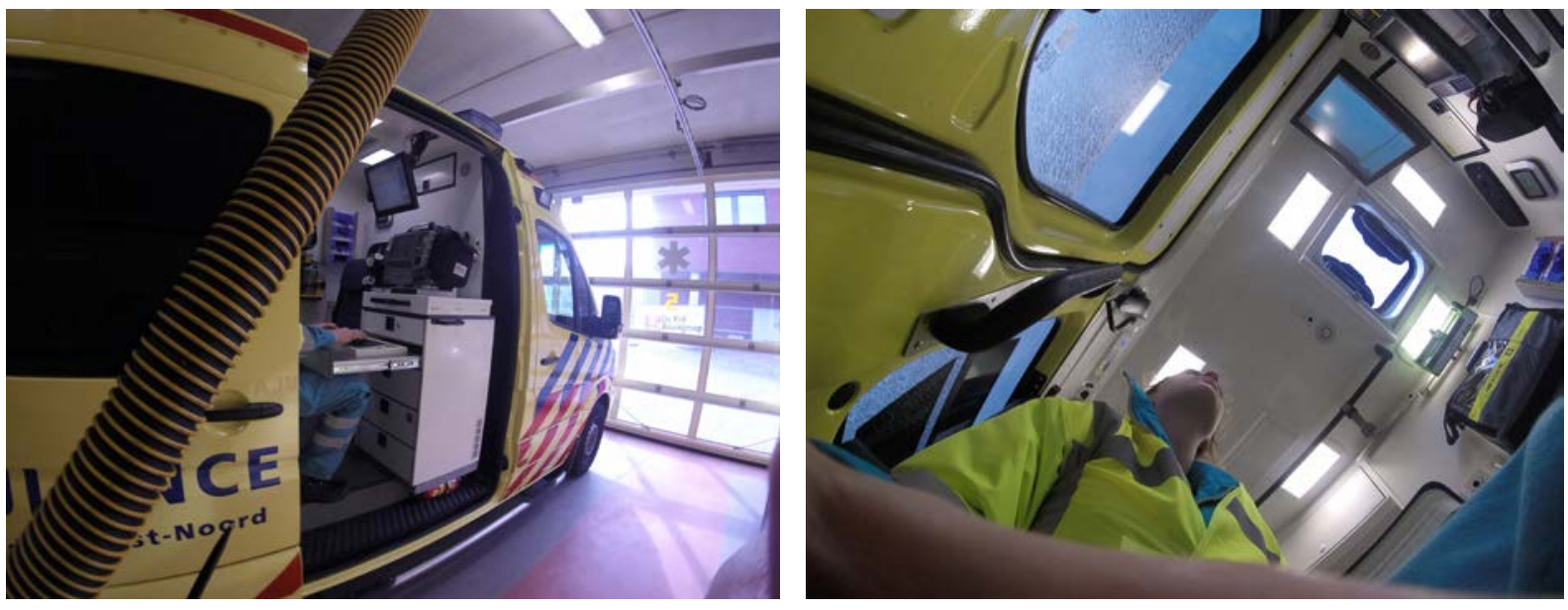
DATA COLLECTION TOOLS
To fit the research specific needs (such
as type of stakeholders, kind of desired
data, geographical positioning, time
constrains) several data gathering methods
were generated and applied. To better
understand the research outcomes
reliability and relevance, an overview on
theircharacteristics and usage is here given.

main resources, being.

main resources, being:
1. The scanning of EMS professional fora and blogs, from which to gather a taste of the professional life of

ambulance workers. In particular

ems1.com, a leading website serving

the Emergency Medical Services

community worldwide and coun

more than 130,000 registered

A set of questions was released

on Quoracom, a website in which

questions were formulated both for

gathering information and for testing

questions formulations, checking the

best ways of receiving interesting

answers. For instance, the question

What is a superpower that could help ambulance paramedics to do their job aenter reved pors creativity and was re-used in the cultural probes

The set of questions and the received

answers are available in Appendix II.

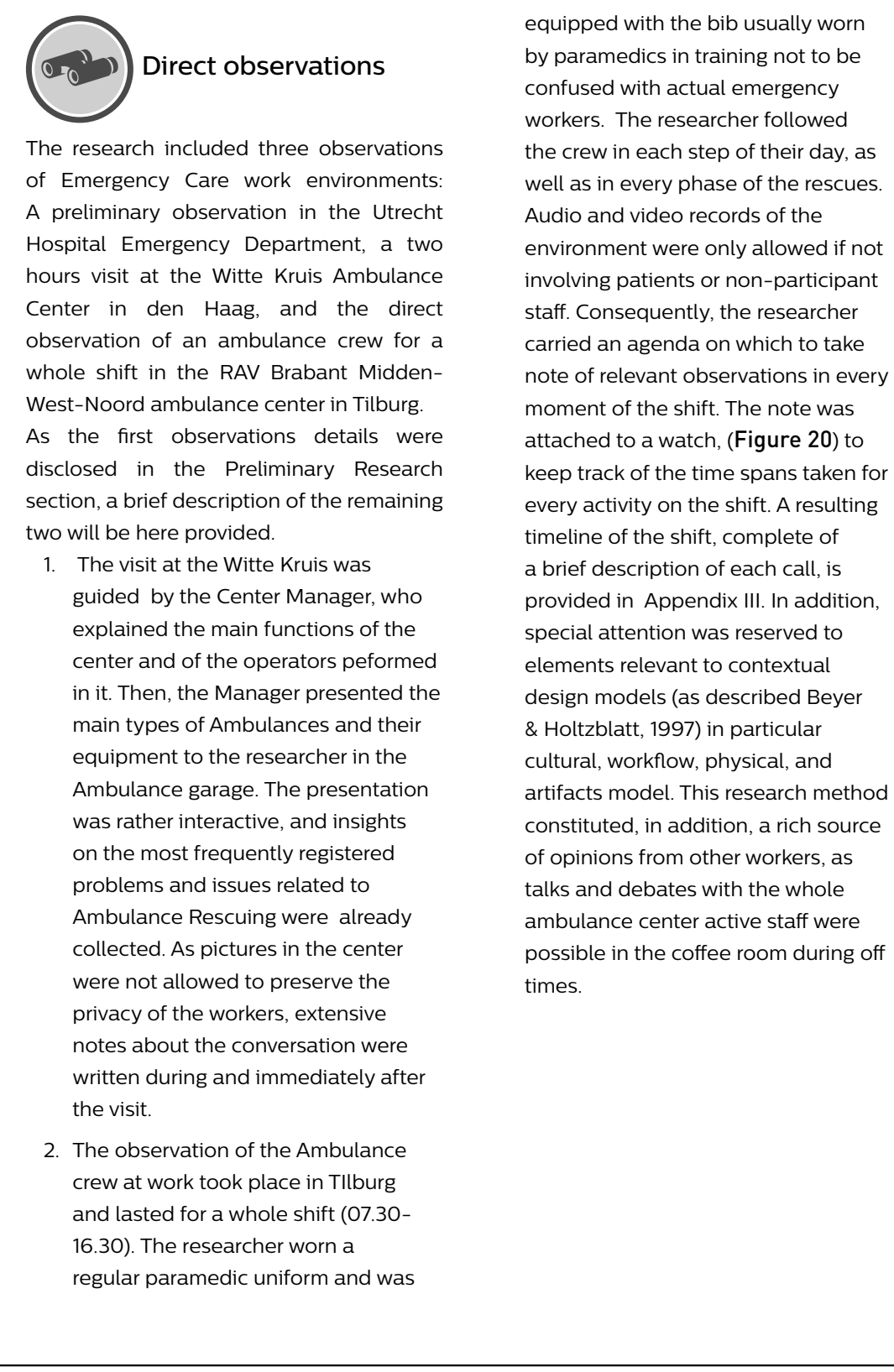

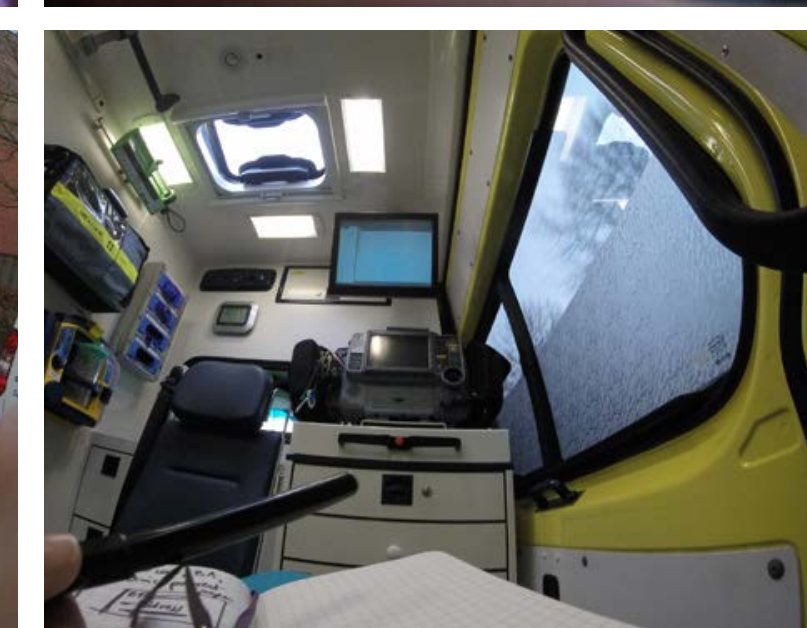

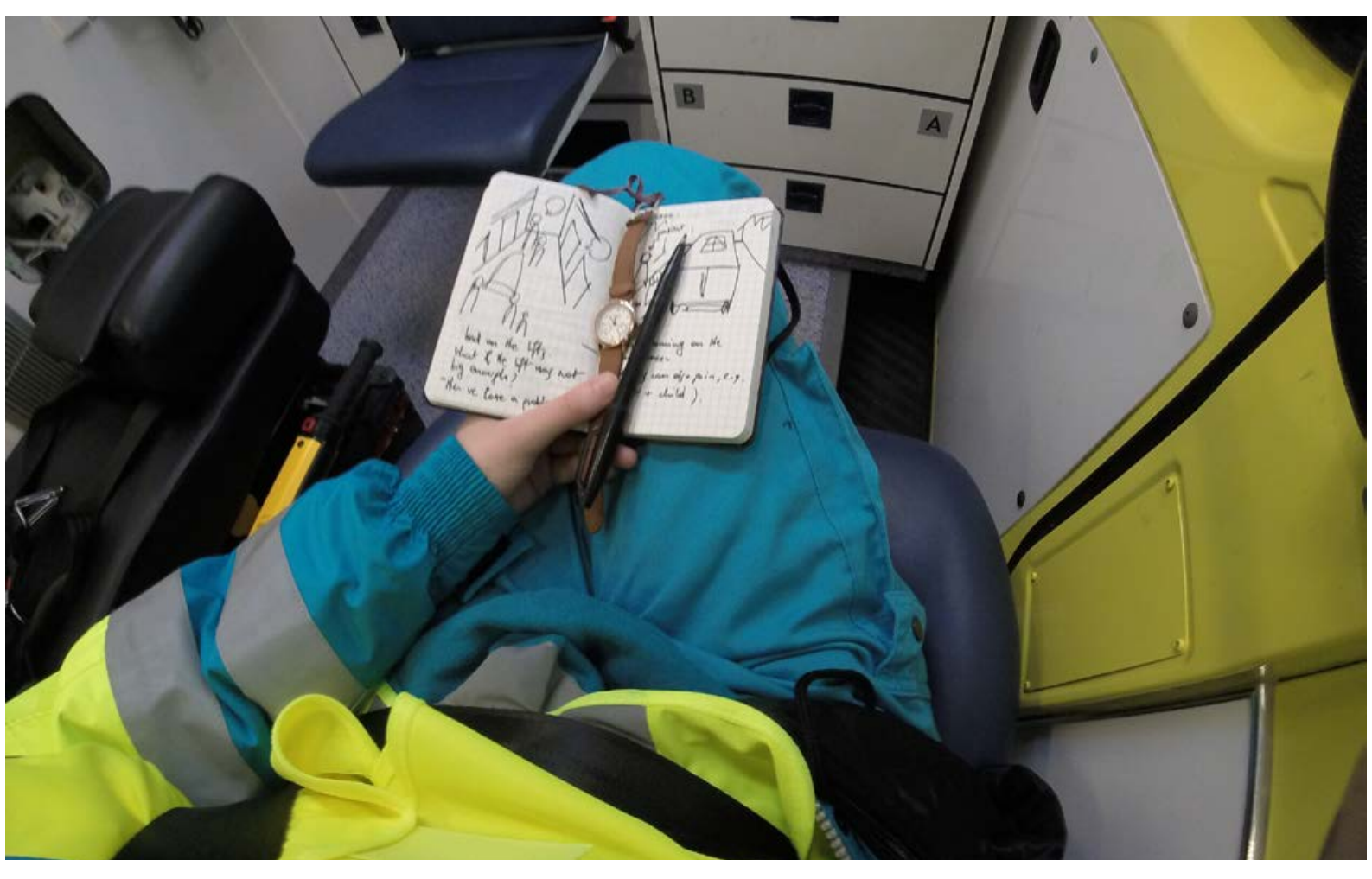

Figure 20 Direct observation of
an ambulance shift 
(D) Cultural probes

Cultural probes, as first described by Gaver (1999), are a user centered research approach reveal users' personal perspectives to enrich design and support empathy.

This method was chosen in consideration of the muttiple advantages for the hereby presented research, as

The adequacy to unveil personal issues as well as tacit and latent $k$ row

$$
\text { The advantages }
$$

participants to

topic, united to optimal adaptability to

the participant schedule - a particularly delicate factor in researching with

ambulance workers.

The convenience for the participants of being conducted in the original language whe parkipans (Dutch), factorthat - interviews no be possh rem

To elicit personal involvement, the formulation of the probe exercises was always made in first
person. For instance, the booklet title was ' $m y$ life as an ambulance nurse', and the five 'exercises (one per day) structured in thematic areas, were named, respectively.
1. What do I do?

2. How do I work?

3 . How do I like it?

4. does it take?

5. What would I want better?

The exercises were translated as much as possible into visual and tangible artifacts stickers enclosed in the bookte. (Figure 21) In total. five booklets were sent by resular mail to participants located in various locations in the Netherlands (Figure 19). Apart from the bookle itself, the envelope included instructions for the use of the probe, researcher contact details, and available in Appendix IV.

The recollection of the probes, which the occasion to perform direct interviews to each participants. The interviews were used as a follow-up on the probe completion as well as a confirmation of the main insights generated by the probes. Also, the interviews could benefit from the application of the event reconstruction schroe, previously recalled by the participant aimed defining the kind of experience felt in that very moment. a pen. The content of these booklets in English method (ERM), a tool introduced by Grube, 2008) and heccupational psychology (Gribe,

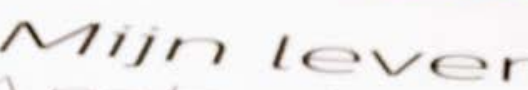

Verpleegkundise
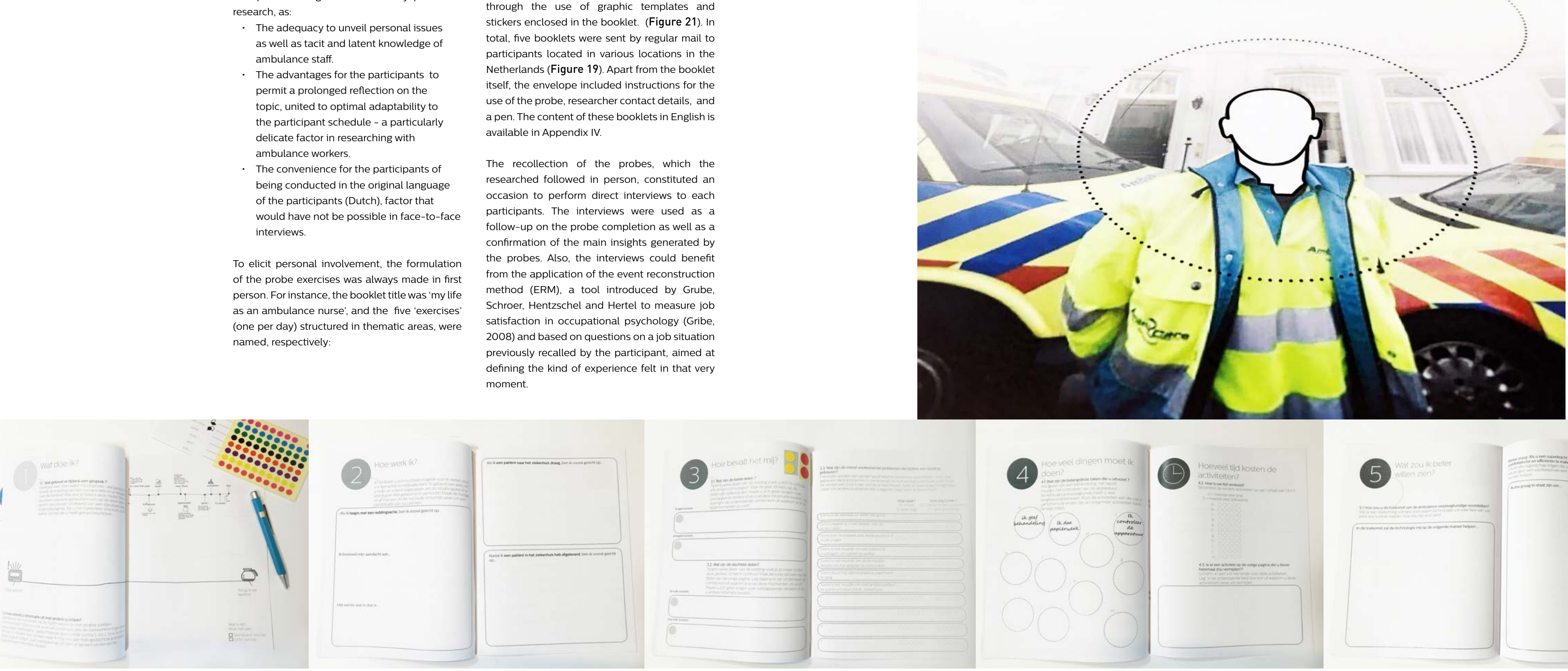

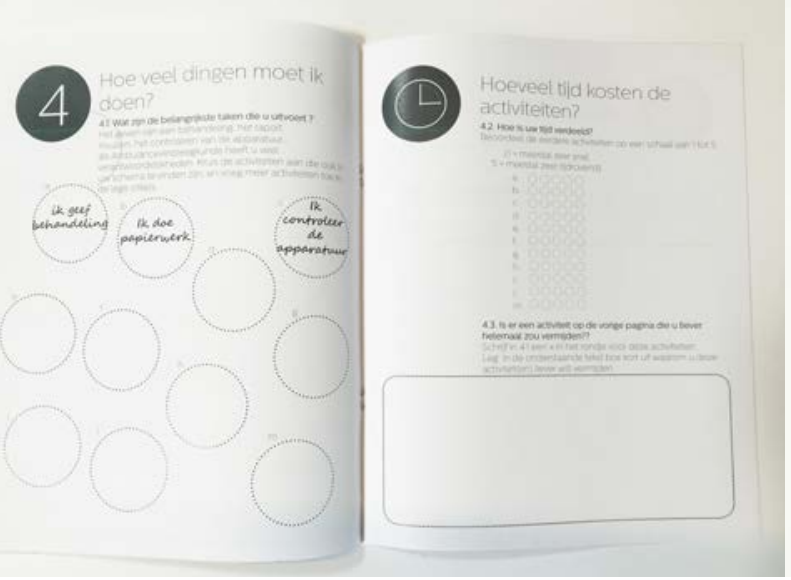

(5) 


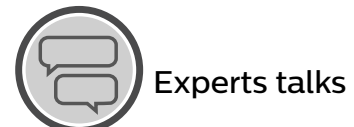

Three talks with Emergency Department expert workers and/or researchers were conducted as a conclusive context analysis. These talks, coming after the field research actives, could benth firm some eanly list of problems) upon which the experts cold provide their view.

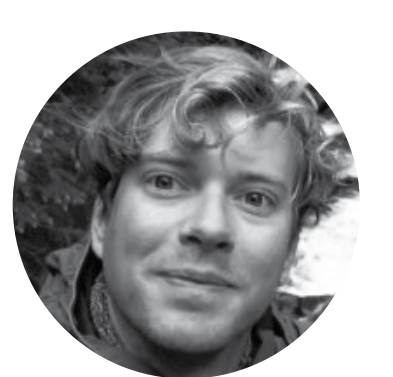

Frank Goethals Healthncave ar iU HCealth \&

Talk highlights

IT systems of Ambulances and Emergency Wards should be able to efficiently.

A direct connection from ambulances to response teams in the hospital could constitute a value from the Ward staff. than providing specific answers to the fescearch with a much 作 minutes of thesese nects. Ex

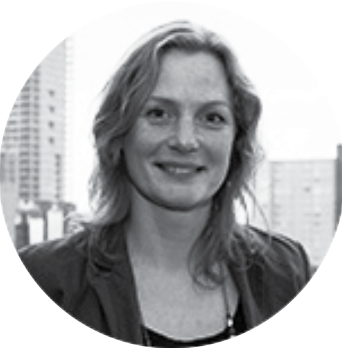

Juanita Haagsma

Researcher $a$ at Erasianmus MC
Hospital in Rotterdam

Talk highlights

Emergency Departments in which Emergency Physicians are usually working face less problems in arranging and managins response teams.

Much overcrowding of the Emergency Ward is caused by dificituties in reariecting stabilized patients to deffnitive care. stable pathents inght have to be transported back home or to other hospitials, but the Melakamer would not allow for Ambulances to be used nnon-emergency cases

"If you want to become millionaire, invent a unified information system that covers all of the emergency patient journey".

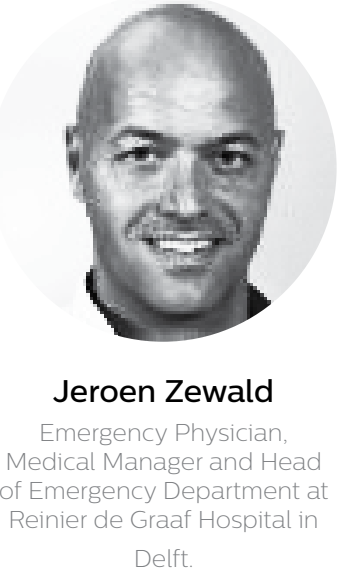

Talk highlights

Face-to-face

communication about the patient state is always

preferable

Pictures of patients ID's if sent in advance to the Emergency Department could speed up administrative procedures percase

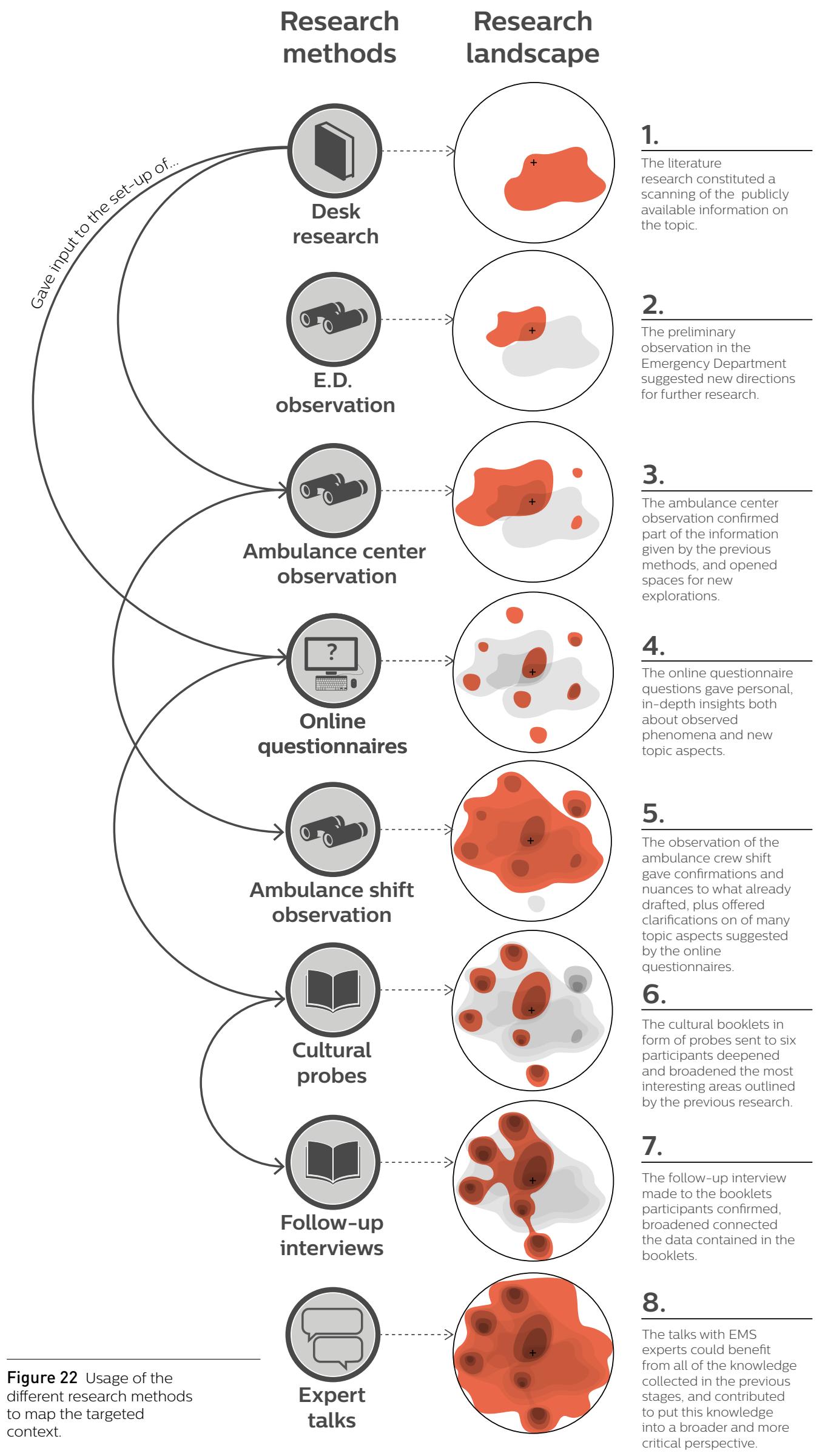




\section{Research results}

Data analysis and outcomes

DATA ANALYSIS

METHODOLOGY

(घ9) Analysis on the wall

Analysis on the wall (as described in Sanders and Stappers, 2012) was selected as the preferred

data analysis method

This was chosen in consideration of the capacity of the technique to be compelling and involving The analysis was divided into three stages:

Preparation

From the previously presented research tools, the following results were printed and kept visible on a wall:

Labeled quotes from the online user research - Labeled pictures and videos from the observations.

- Translated booklets (including drawings and time-lines from the originals)

Labeled franschins from the direct observations. and from the interviews with experts.

In addition, empty templates for characters and rescue workflow were prepared. These templates sheets for 'problems', 'ideas' and 'experience factors'.

Execution

The researcher scanned and reviewed all of the research results. The appropriate pieces of information were placed into the empty experience factors problems ides) In andion the rescue workflow included special insights concening 'data points' or moments whose importance was regarded as particularly mportant for the exchange of information in the rescue workflow.

The whole analysis was video recorded, in orde 政

Elaboration

An additional synthesis process was executed was worked out integrated and refined reaching the form of a research deliverable.

This stage constituted, for the first time in the project, an opportunity to go beyond the descrntive stage and deepenthe research focus. taking into consideration the implicit meanings of the collected data.

in particular, the interpretation and confrontation of quotes concerning positive or negative to the identification of a number of contextual experience factors

All of the deliverables resulting from the analysis on the wall are presented in the next paragraphs
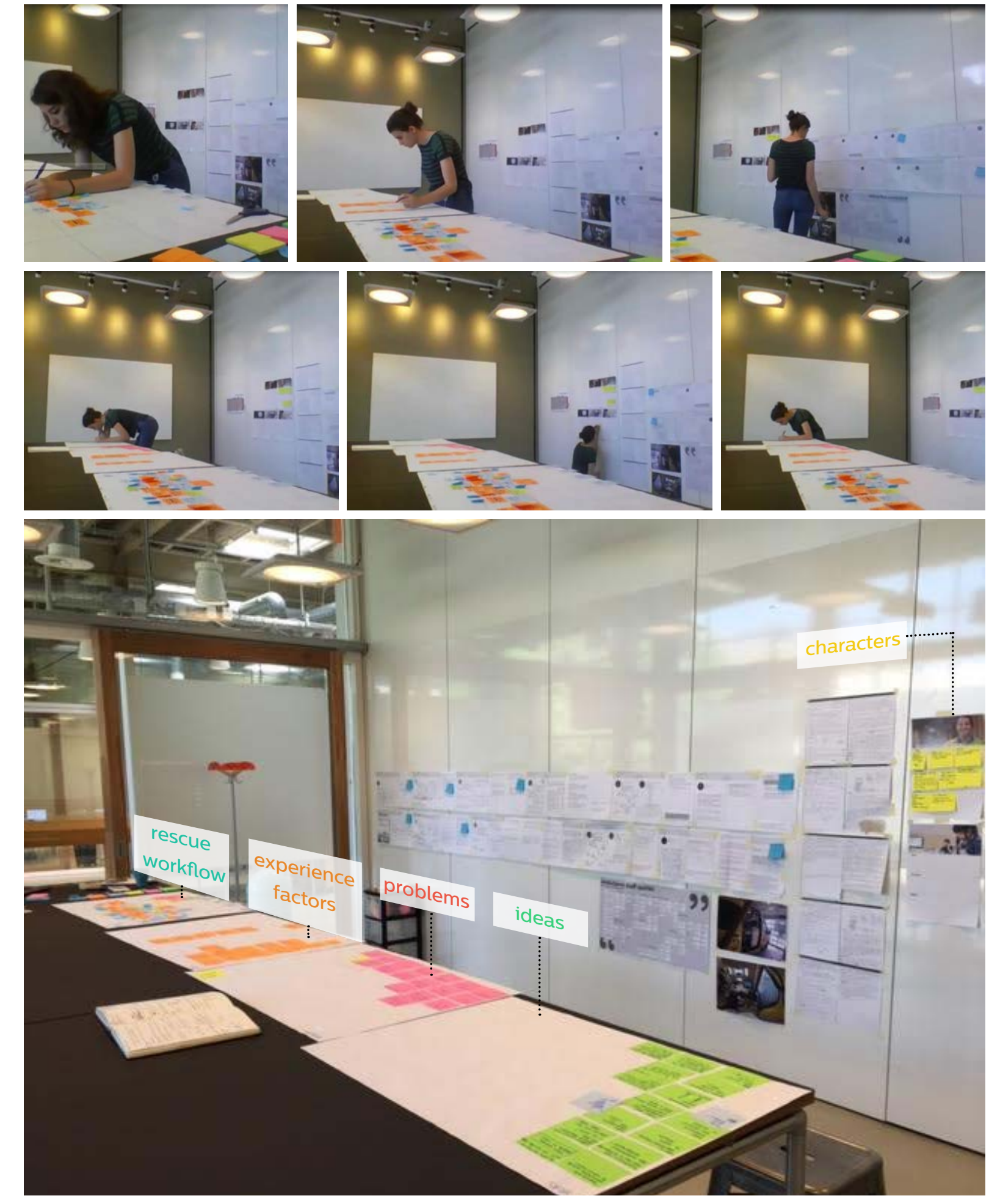

Figure 23 Analysis on
the wall 
RESCUE
WORKFLOW

Model description

Part of the user research results were processed into a model narrating the main phases of an average ambulance rescue
process. The process was described on Several levels, advancing from a simple description of the performed actions to a

The levels of description are:

\section{Stakeholders involvement} Mental landscape

This model is given the operative name 'rescue workflow', due to its feature of describing not a real case, but rather a equence of operations usually performed sequence in ambulance rescues,
disregarding from the type of medical emergency.

With 'average ambulance rescue process' is are excluded from the journey As thes shouldn't be neglected, they are hereby listed in order to be taken into account he further design process

- events are:

Decision of the ambulance nurse to not bring the patient to the Emergency Department (often in practitioner).

Patient refusal to be treated or

transported.

detailed explanation of the graph The last level, describing the nurses sest negalive expentence for the nurses

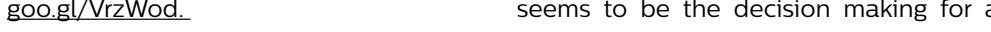
In alternative, the full model can also be visualized by scanning the QR code in this

Model relevance

The goal of the rescue workflow consists in gaining both a rich and broad overview to its narrative structure, it is regard as a suitable support for illustrating the basic functioning
inexpert listeners

In addition, the model is meant to highlight

Main insights

An overview of the overall rescue workflow could bring to a number of contextual corvation

The first ones concern the organization the teamwork among the parties, uncertain case.

Overall, the trend of negative and positive ext mo that same situations could bring to opposite reactions for different individuals and different emergency types. The ride to the sement and adrenaline rush (positive) by some participants, and of the destination scenario (negative) by others. This suggested the need for the good or bad rescue experience of ambulance nurses, which was therefore reserved a separate model (presented late in the chapter).

As an exception for this ambiguity haw the nis tos tesponses, ilisto notice how the tho to the hosplat represents anbulance nurses both for negative and positive experiences.

action, the hospital personnel as a whole operation. The emergency patient transfers, each characterized by a differen

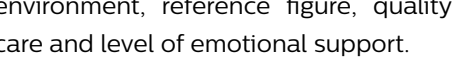

On the second level of the model, it is relevant to denote the large spectrum of communication devices used by the partes

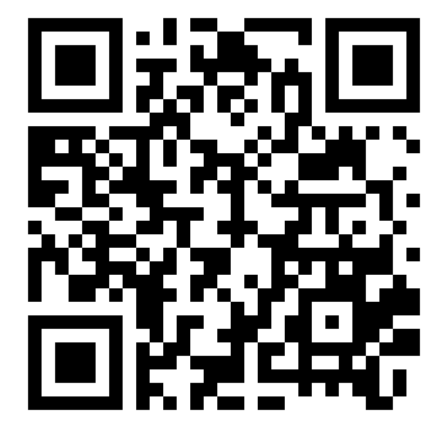

In the level of mental processes, which was escue, while ambulance services ordinantly that nurses are constantly updating ther perform planned transportation (e.g. knowledge on the case and anticipating the next steps.

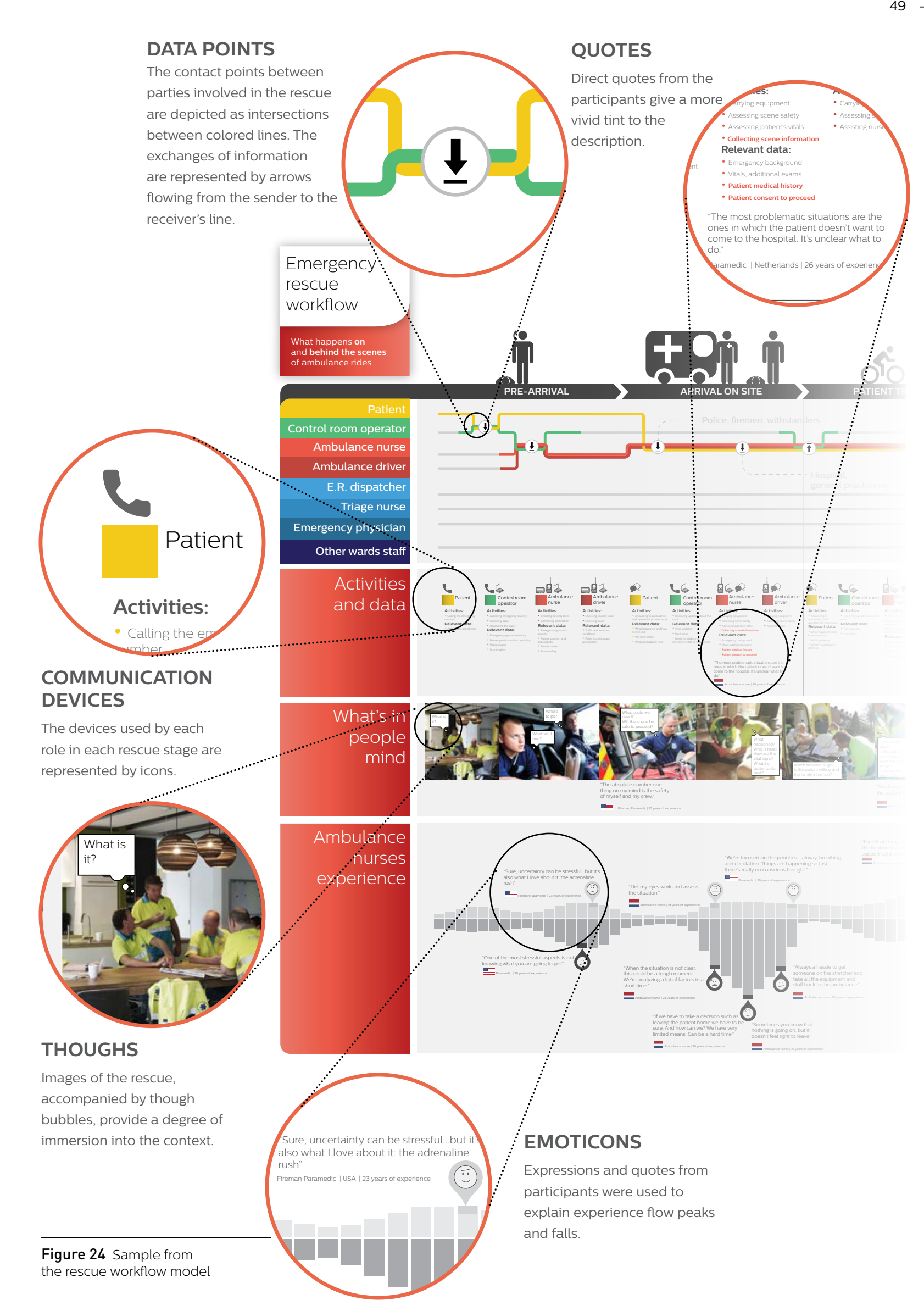




\section{(8) characters}

\section{Model description}

A separate model was chosen to describe the features and peculanties of the ambulance rescues. hsights generated from several research methods, in particular direct observations of the driver-nurse partnership and from direct interviews with experts

\section{Model relevance}

The characters descriptions include the professional role's key responsibilities, (n)

character with others is provided, to give a in particular, a breakdown of influence is emergency care domain

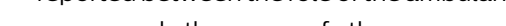
purse and the one of the emergency physican or the trage numses, especially Qut the from the participas are intain the in the hospital, or when the expectation of reported fundings This model will be the paramedic of being carefully listened thes choices for is not fulfilled Not unequivocally positive instance regarding possible desirable side are also the feelings of the ambulance $\begin{array}{ll}\begin{array}{l}\text { The five characters outlined with this } \\ \text { method are presented for in the next pages. } \\ \text { nurses. }\end{array} & \begin{array}{l}\text { nurse towards different from ambulantrol room operator } \\ \text { sometimes defined as 'distant from the }\end{array}\end{array}$

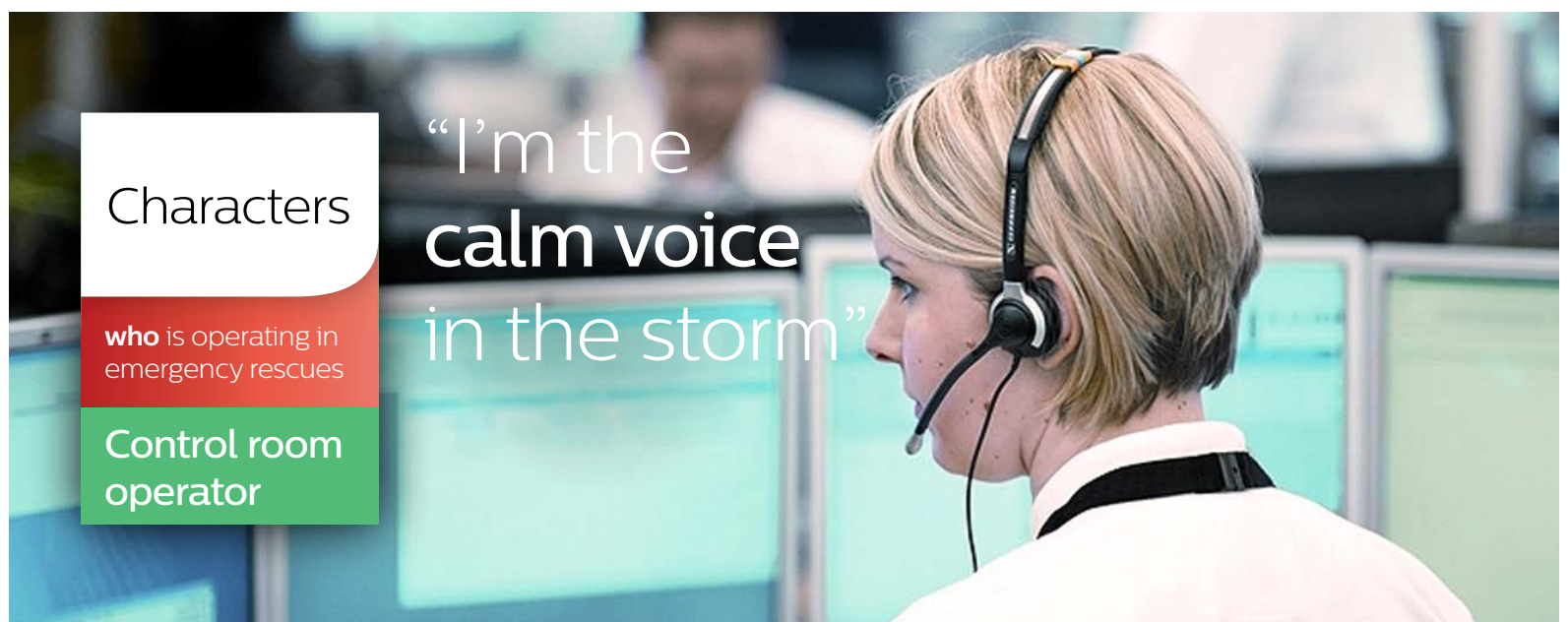

\section{Key responsibilities Responding to the emergency calls
and directing the rescue operations. Collecting all of the initial patient location and accessibility personal details \\ Keeping track of the ambulances}

\section{Key qualities} Fast thinking Emotional ș
Multitasking
Goals and motivations Adrenaline, sense of responsibility highly complex systemag, backgroun

Wishes

\section{- Automatic identification of mobile}

phones position Automatic translation from every
Stress sources

- Unclear patient location or emergen

type
- Language barriers
- 'Frequent fliers'

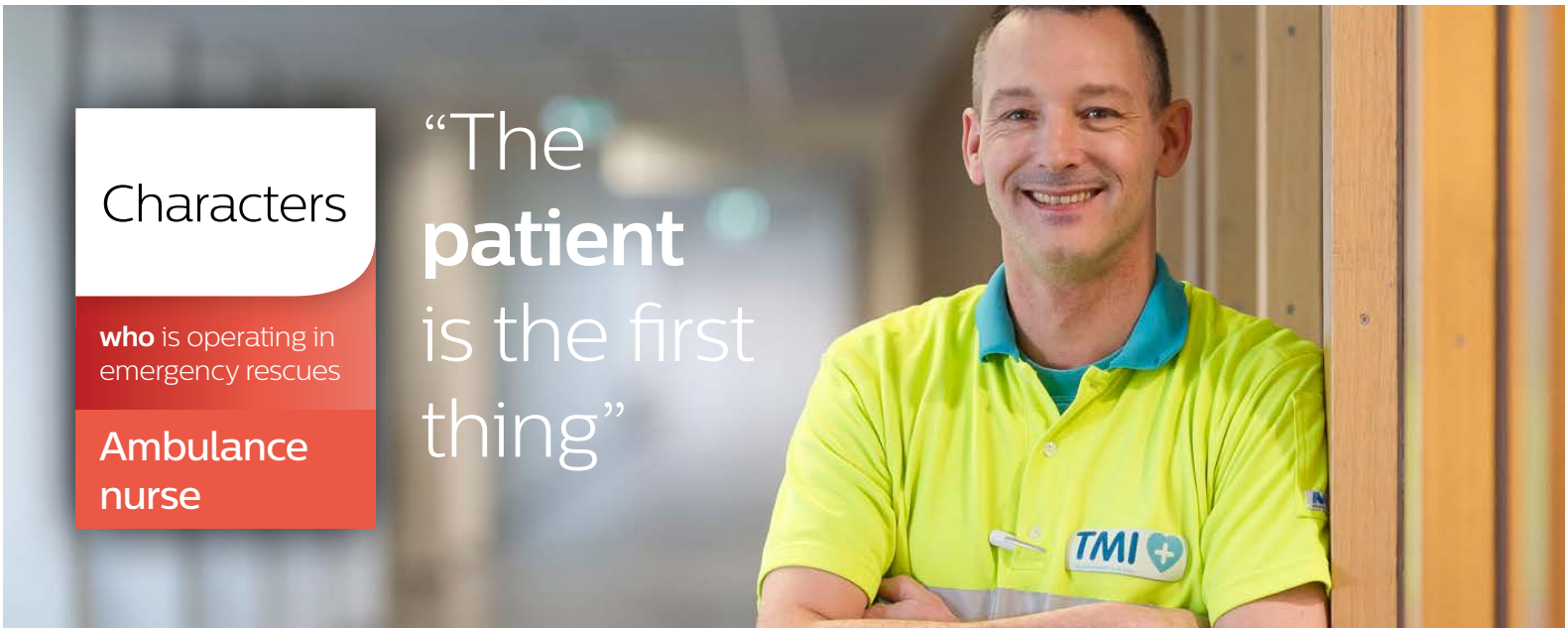

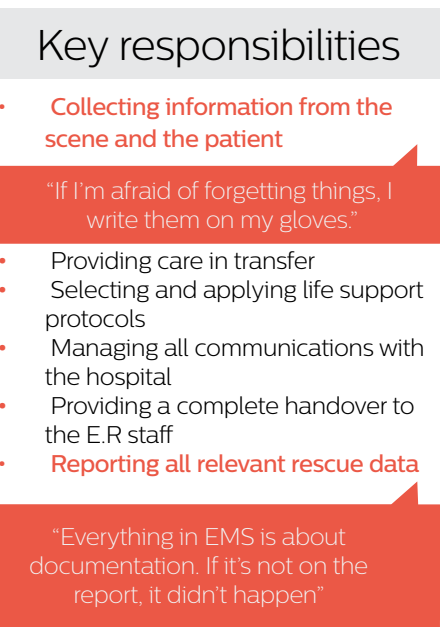

Key qualities

Problem solving mindset

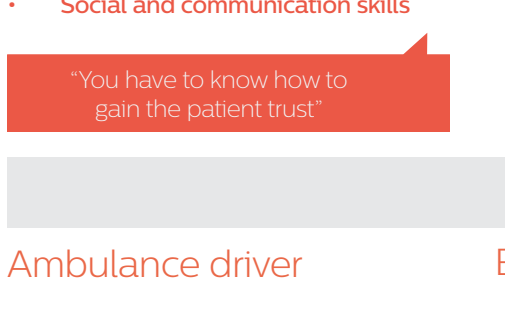

Ambulance driver

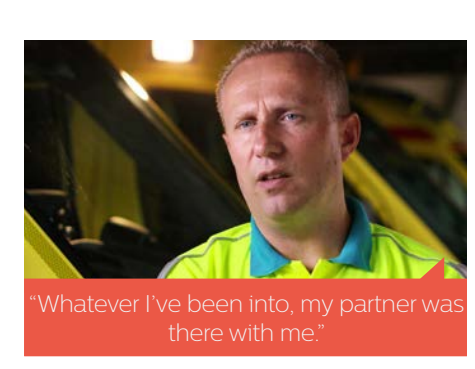

Goals and motivations

+ Freedom, adrenaline, working with the

people
+ Special relationship with colleagues

+ Offering need it the most

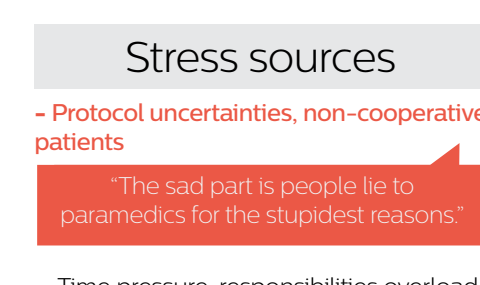

- Time pressure, responsibilities overload
- Time wasting inididents lensthy procedures
Traumatic events, physical distress - Traumatic events, physical distress,
mental burnout

About others Emergency physician - Arelief from responst
source of concerm

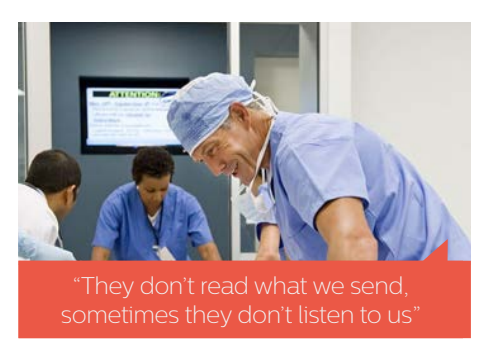

Wishes

- Faster, more flexible bureaucracy

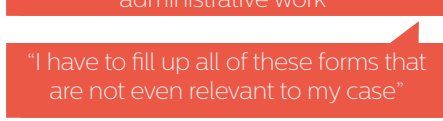

- Reliable, real time data collection Easier access to patien information

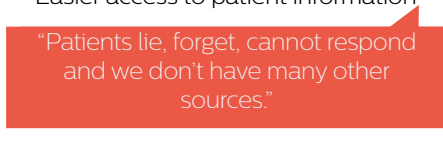

- Easier decision making communication with hospital and control - More advanced and complete diagnostic equipment Clear tools for assessing scene safety - Improved legal protection

\section{E.R. dispatch nurse}

- Usually a flawless cooperation
occasionally a reason of overload

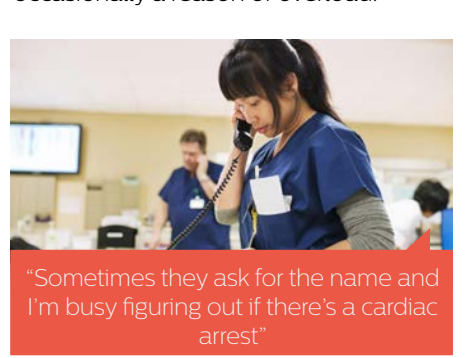



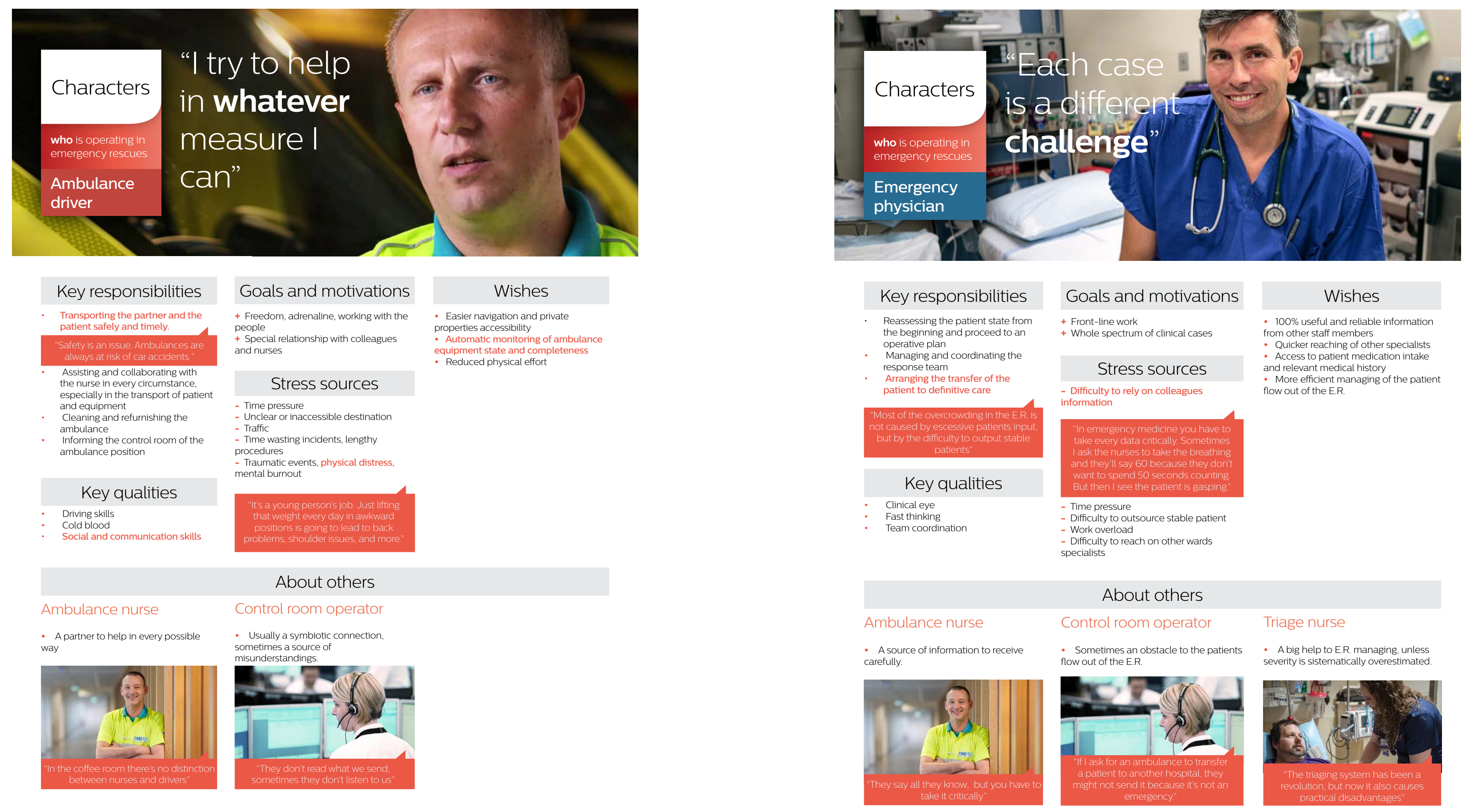


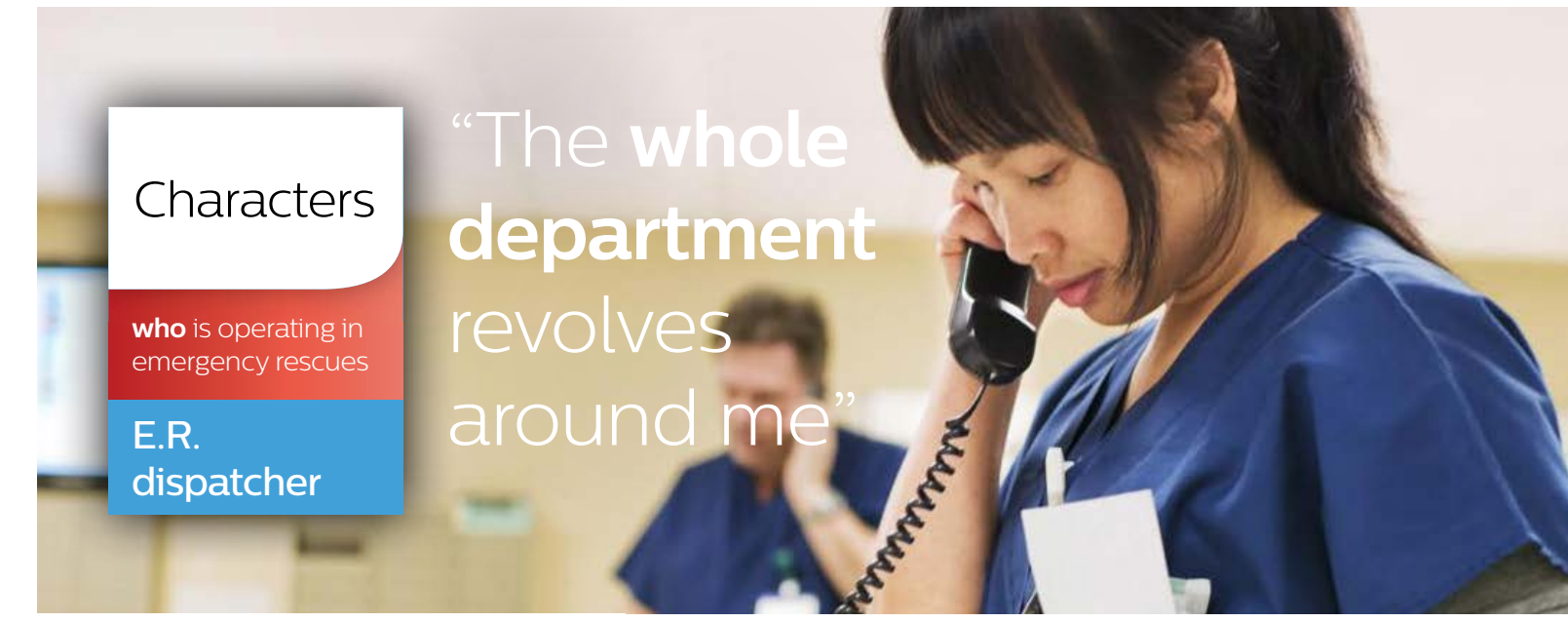

\section{Key responsibilities Receiving ambulance call and
managing the patients arrival in the
$E R$.

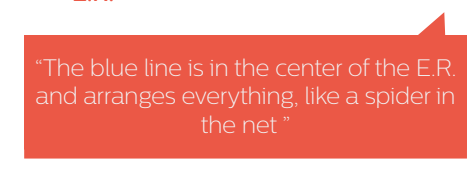

Receiving calls from family of
transported patients or missing people.
Starting patients administrative
procedures

Key qualities

Multitasking
Organination and planning
Social and communication skills

\section{Goals and motivations}

+ Front-line work
+ Exploiting nursing experience on a big
scale

\section{Stress sources}

- Time pressure

- Infermational overload
- Unclear or incomplete information from

the ambulances
- Traumatic events, physical distress,

\section{Wishes}

- Clearer and faster information from

the ambulances
memicker reaching of E.R. staff
members

\section{(C)}

Model descriptio

The different tasks performed by ambulance nurses were examined from the point of was mostly buit after an exercise in the

waltura probe asking participants to rate

their tasks in regard to time consumption

and to select the one tasks they would have liked to shorten or avoid. Thus, more than reflecting a reliable quantitative data set, this model is meant to highlight the nurses percelved tasks duration, and their

attiude towards some of hem. For this

eason, the travel the (to the center or

being practically independent from the

Taking the

$$
\times
$$

perceiv

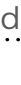

Providing treatment

Monitoring

reatment's effect

Providing care in

transfer

Cleaning and

refurnishing the

ambulance

\section{odel relevance}

Main insights Time management constitutes one of the The first impression given from the model
most crucial priorities of emergency care as is presumably the prominence of the a system. reporting time as the most time consuming

As outlined in the "Trends and directions"

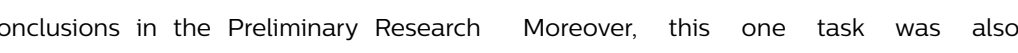
systematically recognized from the 列 Close to it the activity of checketsend to nurses view on relevantly assessed as excessively long and different tasks. This will be used to displeasing. apropriate activities to target for efficiency provements.

Conversely, activities such as communicating with the patient, or providing treatment on scene and in as and genthy regarded as gratifying.

Communicate (with patient, partner,
control room)

Completing the report

Updating

wledge and skills

Figure 25 Time consumption of

ambulance nursing activities as
perceived by involved participants 


\section{두}

COLD BLOOD

I like to be able to think clearly

Not being able to focus is paralyzing when no one else can

"What l love is the problem solving mindset" great feeling" great feeling
"When something bad happens, I feel a sense of
urgency to work hard and fast to get them back, but

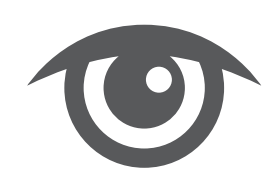

"A superpower that would really help paramedics is

RESPONSIBILITY

Being personally responsible for
someone's safety feels great.

"It's nice to have real stakes, real risks in your life"

"A person without struggle, without a reason to strive to
perform their best is a depressed person."

"Taking care of a truly sick or hurt person can be very
rewarding."

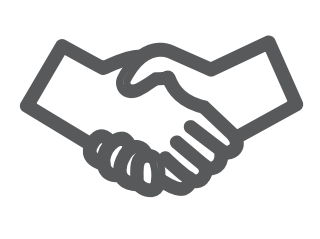

consequences.

"W're personally accountable for all that happens"

gaainsta lawyer who's questioning your methods" "It's a sad truth, but most of us are taught to document
to defend ourselves in court more than to plainly state
patient condition and care"

EVALUATION

Seeing how good I am at doing my job makes me very proud.
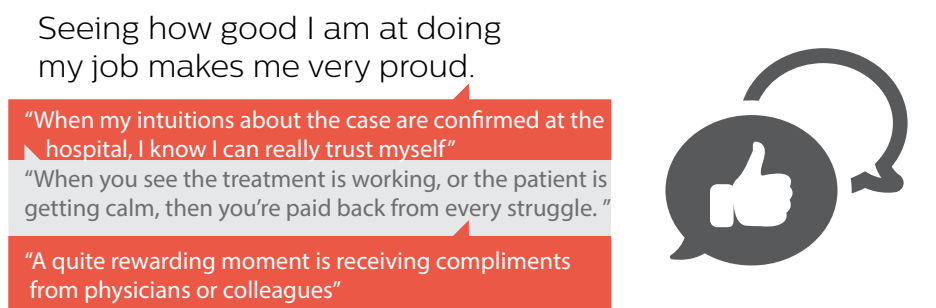

We don't always know if we did the rig thing

"Physicians are busy and often not open to tell us more "lf something goes wrong, we begin to doubt ourself.
Did we respond fast enough? Could have we done "Sometimes we rerelect about recent rides with

PERSONAL RELATIONSHIPS

I love the contact with the people.

\section{"The relationship with the patient is one golden
thing about this job" "There is a huge amount of trust and understanding in

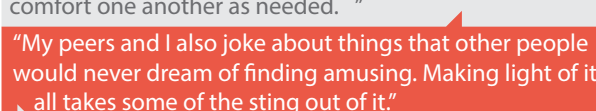 all takes some of the sting out of it." 'colleagues' they ar more ny fonily than mine ativs"}

\section{hen I have a clear plan,}

everything feels ok.

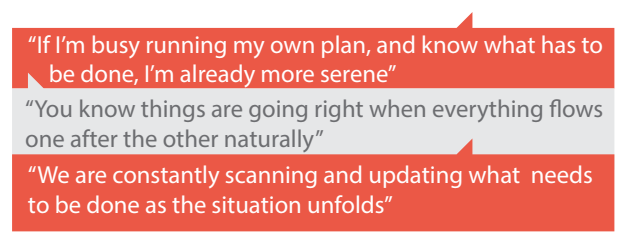

Dealing with the others can be a big issue.

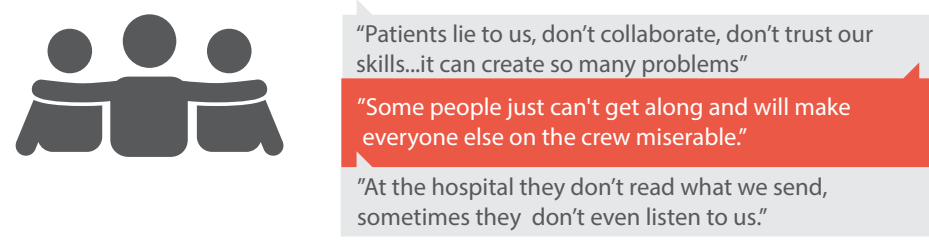

FORESIGHT

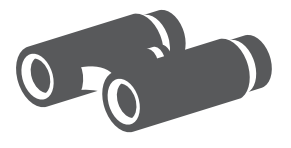

Not knowing what's coming is draining.

"One of the most trtessful aspects is not knowing what
you are going to get.". you are going to get."
"So many times it looks like it's safe to leave the patient
home but then we don't know what could happen in home, but the
half an hour

"You know in fifteen minutes everything could change"
(2) Experience FACTORS

\section{Model descriptio}

As anticipated in the Rescue workflow paragraph, a separate model was reserved to positive and negative experiences of ambulance nurses while accomplishing their job.

As described in the data analysis methodology all of the relevant data concerning nurses expeniences from the various research sources were grouped on a same support and analyzed

This led to a first division into positive and

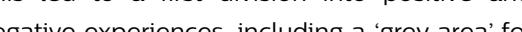
ambiguous or ambivalent ones

This main division was further worked out into an implicit interpretation of the elements determining the quality of nurses expenience during their job. These factors were grouped enamed and recombined until five fundamenta areas emerged, collectively forming a positive or negative experiences

\section{Model relevance}

The model was built in order to get a less The mode a less hurses most desirable experience goals.

These insights will be later used to evaluate future concepts expected desirability from the point of view of ambulance nurses, an will constitute the ground on which to build a

\section{Main insights}

The map of the main discriminating factors describes a complex and somehow ambivalent mindset.
Some elements, such as 'cold boold', 'evaluation', or 'foresight', are essentially organized along of these qualities seems to result in a good and satisfying nursing experience, while their absence could lead to unpleasant, stressing or inadequate outcomes.

Other factors, such as responsibility and personal relationship, don't have an inherent postive sign, and here ferect on nurses mindset seems to follow a more complex set of rules. noted to be one of the most recurring themes in personal interviews and online user research. this complexity is particularly evident.

The direct, personal responsibility felt by the nurses over the patient's wellbeing state was reported to be a thriling, motivating and empowenng element constituing one of the greatest reasons for the nurses to love and consecuences of this responsibility being the personal and legal accountability for the actions performed while on charge (see this chapter's story), was identified from the nurses as their first source of fear, stress, and anxiety

The seemingly unresolved conflict contained in this element will be carefully kept into

\section{The story}

if it's not in the

report it didn't

happen"

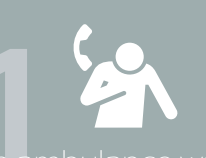

אחי

$-42$

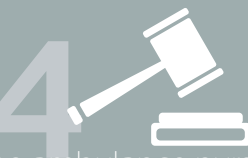




\section{The}

\section{quotes}

things come

one after another

naturally

(

$6 G \square \rightarrow 0$

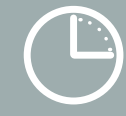

"Q (r) FLOW IN NURSING

Premise

The concept of 'llow' describes, in the realm of human-computer interactions, a particular subjective state observed in users durng the of flow was first theorized by Csikszentmihalyi in the late 80's, (Csikszentminalyi, 1989), but the interest and consensus of the scientific community towards this framework permitted an evolution of the researcher and psychologist work until our very days.

In essence, the state of 'flow' while accomplishing a task is described as a feeling of being in control of our own actions, masters of state of 'flow' is also often corlated with an optimat experience, or the ideal emotional state overcoming both anxiety and boredom.

This theoretical framework is believed to promisingly fit and expand the theme of desired interactions in ambulance nursing, a domain in which the anxiety-boredom balance, and the impontance of control and mindrulness, has already been emphasized. Therefore, an designed for the context of ambulance nursing and keeping into consideration the relevant experience factors outlined in the previous chapter, is proposed

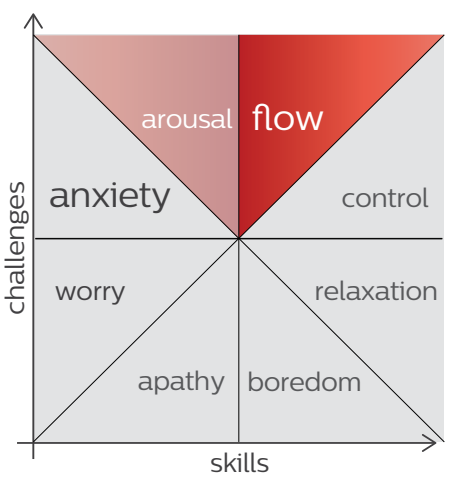

\section{Model description}

intense concentration was in fact ascertained by the research of the direct observations. After a preliminary ent's house, and a consequen decision to hospitalize a patient, both the nurse and the patient were observed to evolve coop way or acthn into a very fuid, seamless accorticts simultaneousty (providing medict set of task (a) and more), while maintaining a visible serenity and apparent effortlessness that brought him to the point of whistling.

In more indirect sources (booklets and online quests), the ideal stuation of focus was often described in terms comparable to the traditiona now framework (see this chapters quotes). From his set of sources, a model was bullt to identify deal mental state as well as the fictise that can render this mindset impossible and conduct to anxiety In Csikszentmihalyi the six characteristic of flow are listed as focus, ecstasy, inner clarity adequate skills, serenity, timelessness, intrinsic motivation (TED.com. 2016). Some of these factors do find an equivalent in the realm of EMS, although declined in different shades For instance, in aplaceable enablers of this pe experience and self confence (- seem skills), intrinsic motivation (as in desire to help or nursing vocation). and clarity of mind (related to the cold blood experience factors an resembling Csikszentmihalyi's serenity)

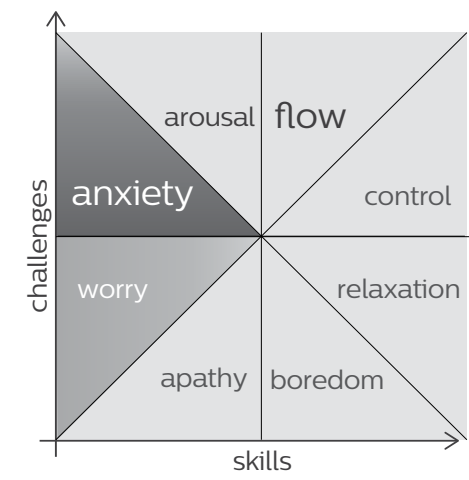

Figure 26 Flow and arousal can be accessed when challenging situations
adequate users skills.
Figure 27 Anxiety and worry are both possibilities when
users skills are consciously
Next to these enablers, a number of Especially regarding this point, the Together with the set of experience factors, contributors have been isolated stimulating observations collected in this research it will contribute to define and steer the (n) design now. In particular, these elements have contribution on, the classic flow theory been defined as the good relation and A deeper treatise of the relation of the
communication with the partner, the present research with Csikszentmihalyis communication with the partner, the present research with Csikszentm patent and he ohnering

in the patient recovery from the medicat emergency.

\section{Model relevance}

Finally, a description has been provided of The model will be used to shape the future vision of the nurse interaction with the and in the communicative and behavioral style.

\section{Main insights}

The presented observations highlight the motance of training, self trust, motivation outcome in emergency rescue practice Additionally, the model confirms and casts new light on the prominent benefits of efficient communication within the practice of ambulance nursing.
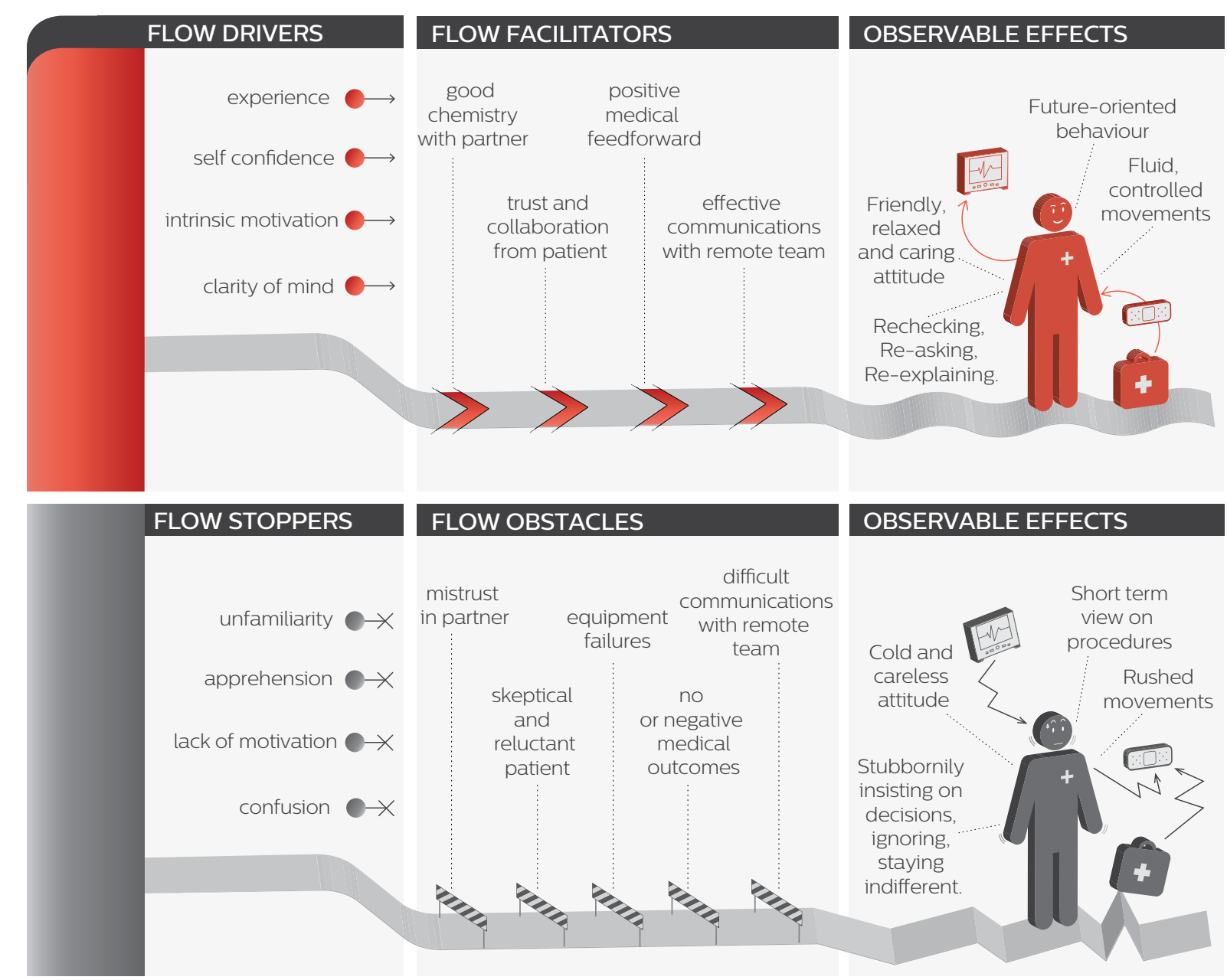

Figure 28 Factors

determining the reach of $\mathrm{a}$
state of flow in ambulance 


\section{User research methods}

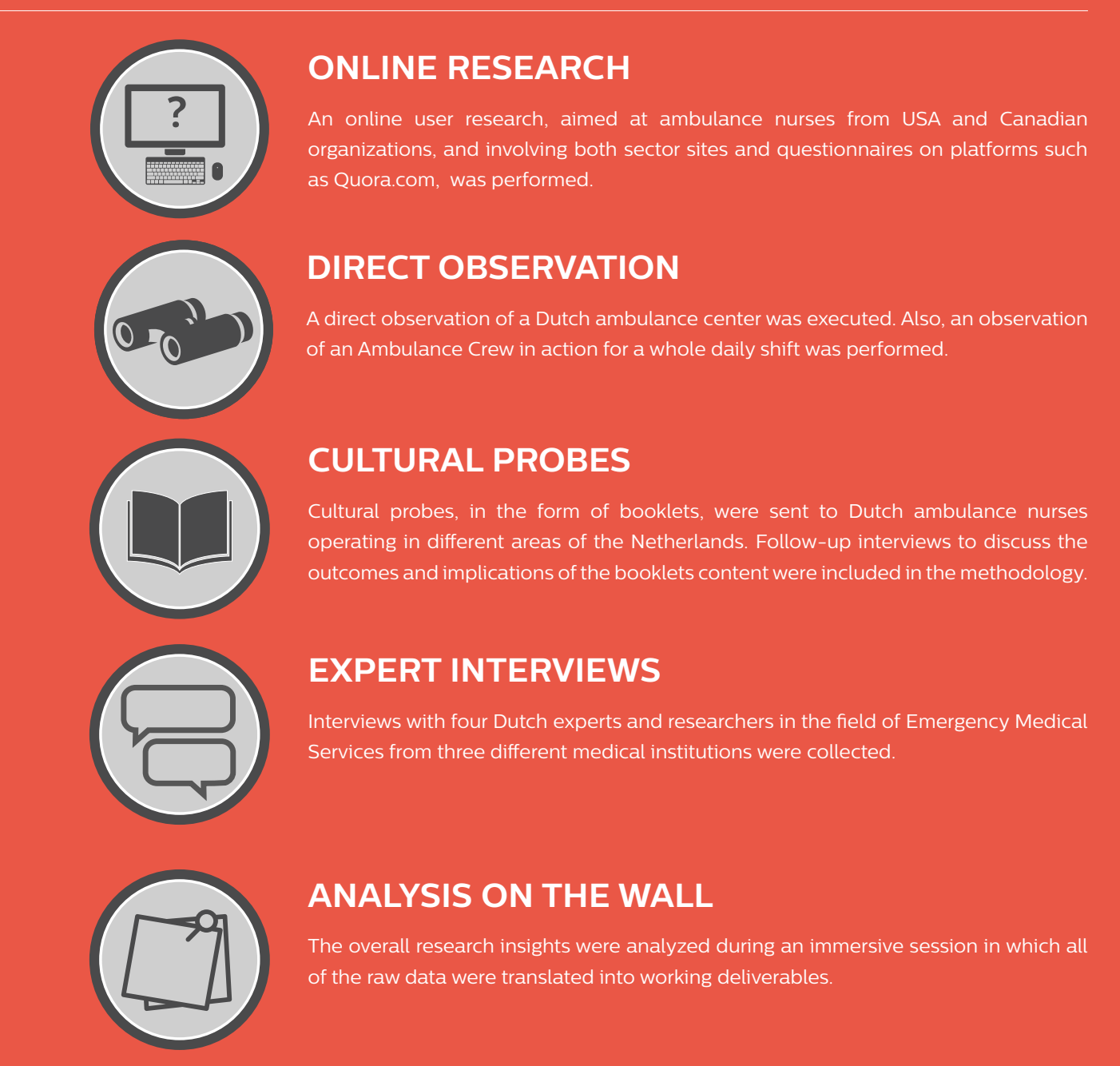

User research theoretical framework

FLOW

The concept of flow, defined in human-computer interactions as a particular
subjective state observed in users during the accomplishment of certain activities
was adopted as a relevant framework to shape the desired future interaction. Was adopted as a relevant framework to shape the desired future interaction.
A customized flow model, describing the ideal state of focus of ambulance nurses in the accomplishment of their duties, was built. 


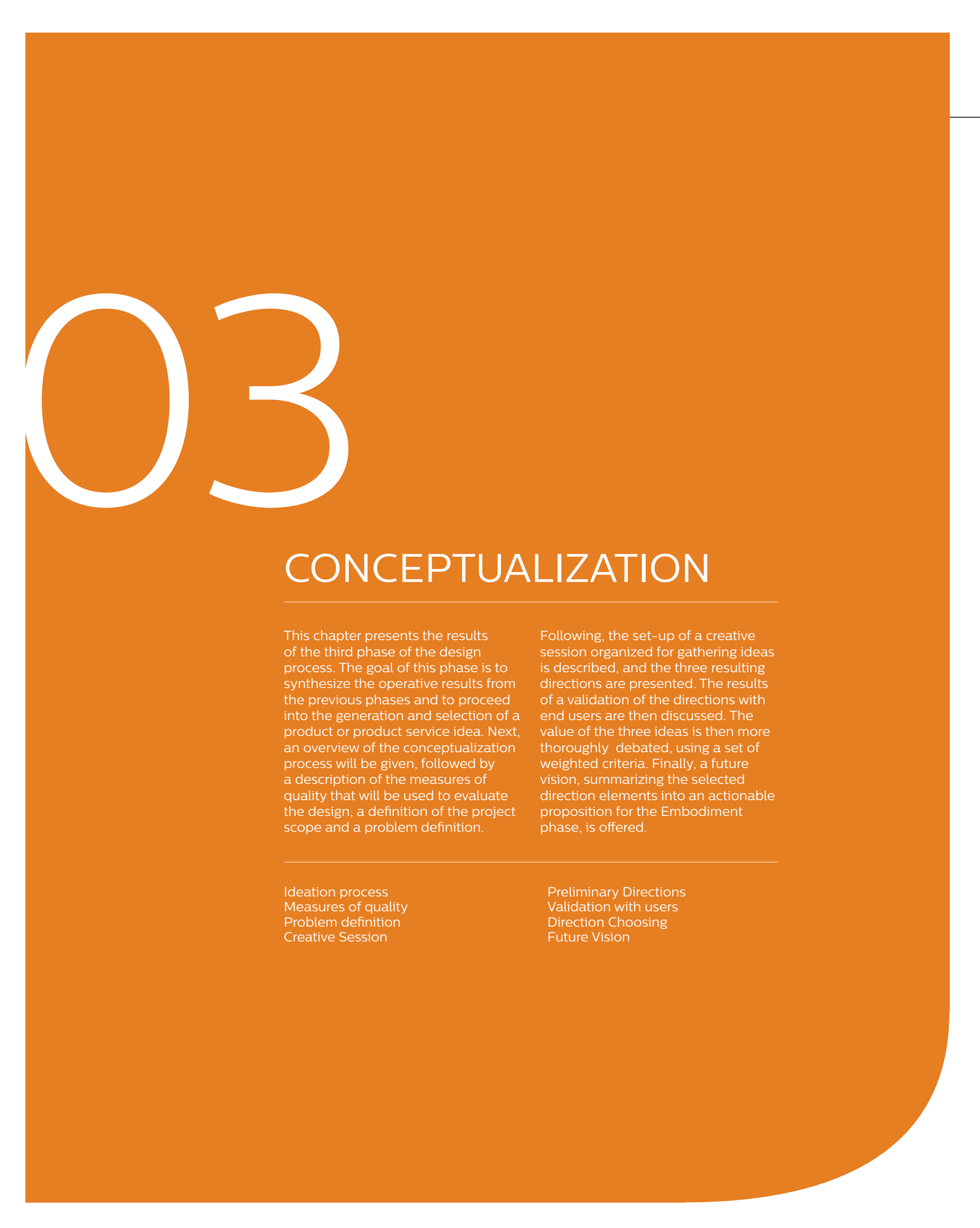




\section{Ideation process}

Guiding creativity

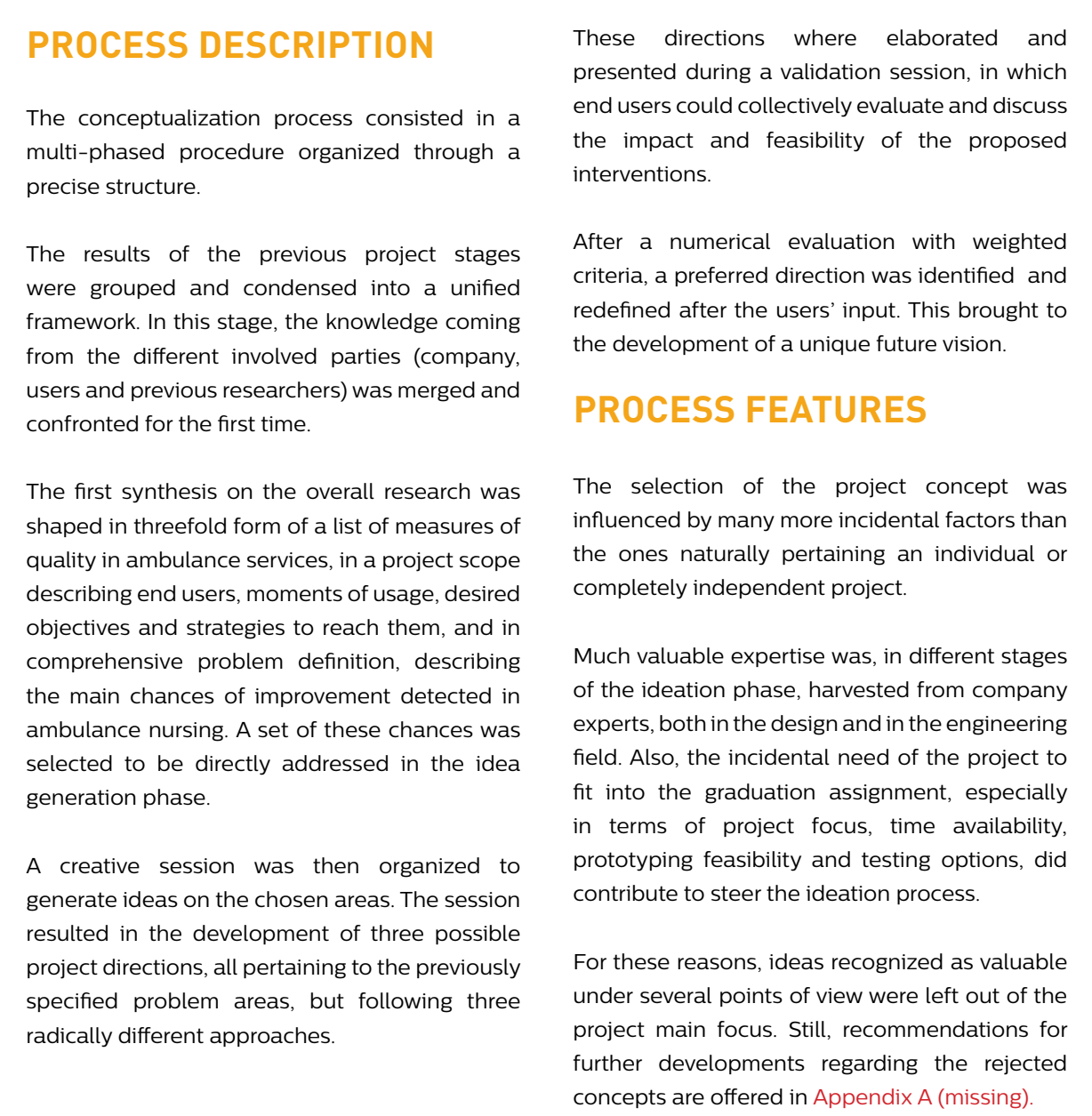

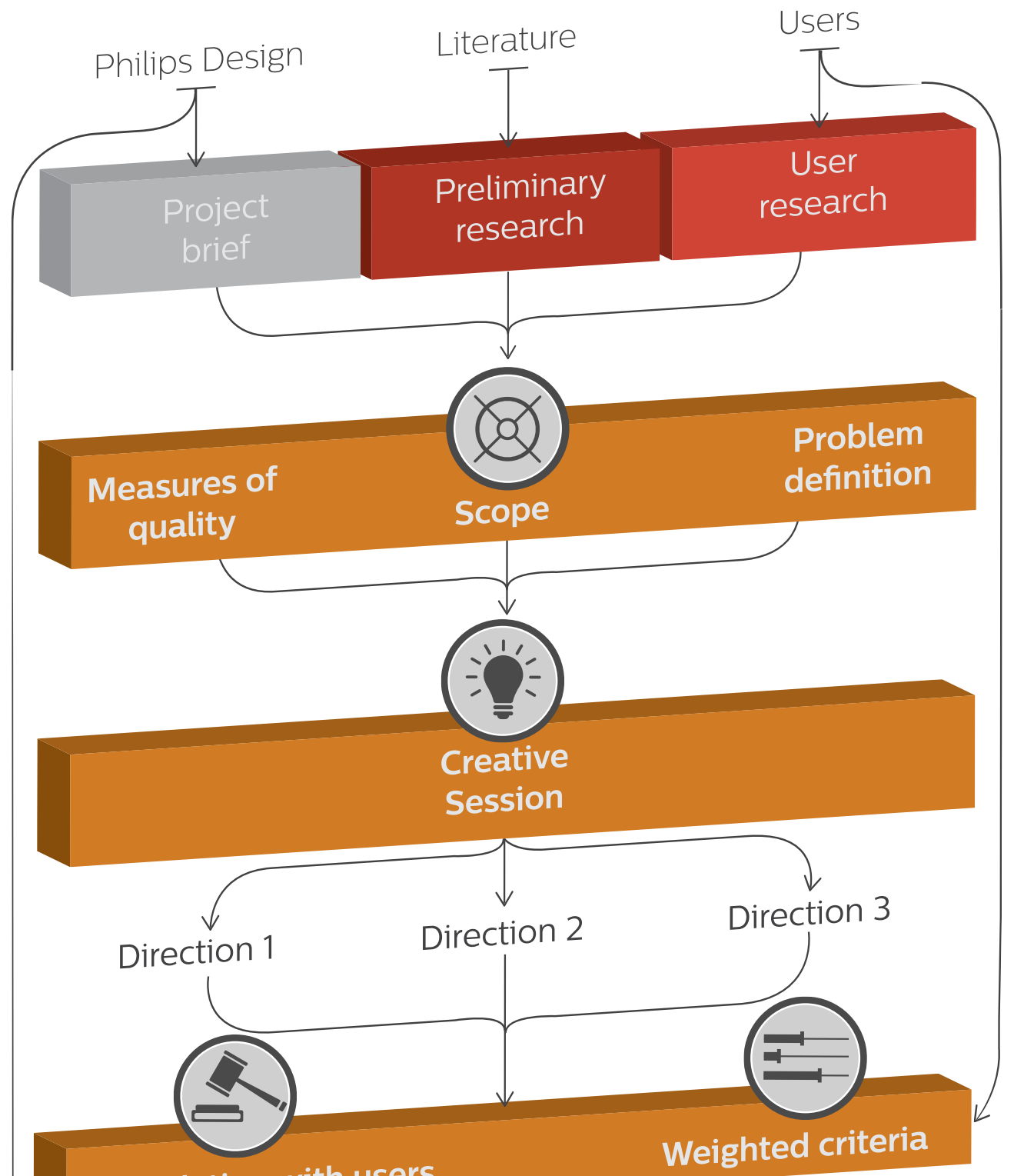

Validation with users

Weighted criteria

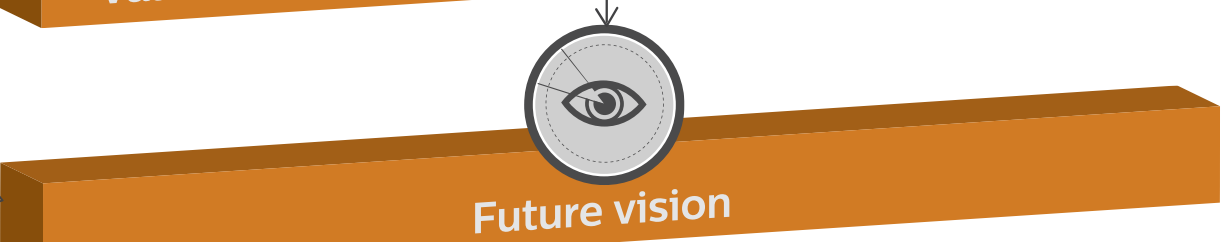

Figure 29 Structure of 


\section{Measures of quality}

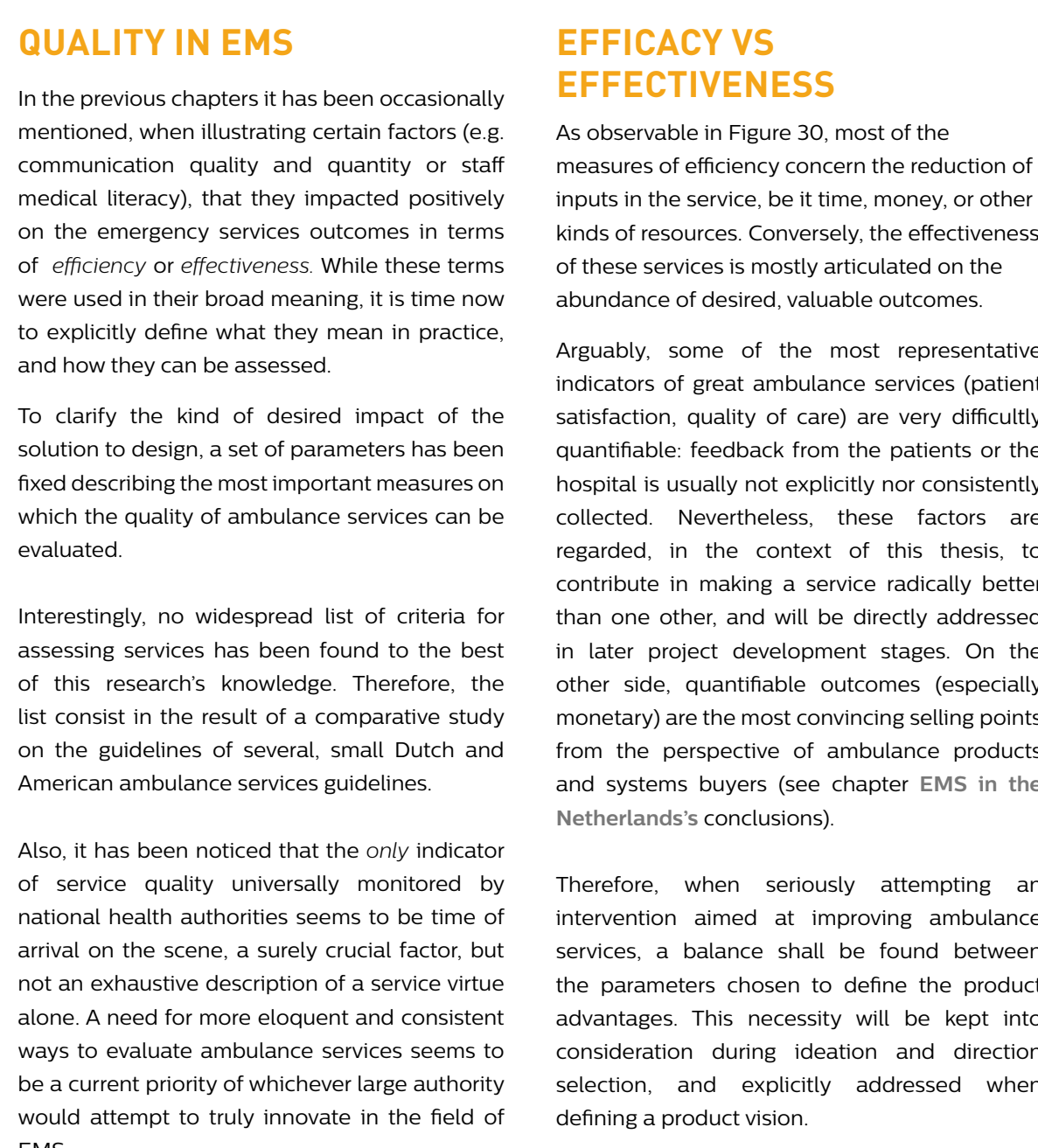

\section{QUALITY IN EMS}

In the previous chapters it has been occasionally en illustrating certain factors (e.g. medical literacy), that they impacted positively on the emergency services outcomes in terms of efficiency or effectiveness. While these terms were used in their broad meaning, it is time now to explictly define what they mean in practice,

To clarify the kind of desired impact of the solution to design, a set of parameters has been which the quality of ambulance services can be evaluated.

Interestingly, no widespread list of criteria for of this research's knowledge. Therefore, the list consist in the result of a comparative study on the guidelines of several, small Dutc
American ambulance services guidelines.

Also, it has been noticed that the only indicator of service qually unversally monitored by arrival on the scene, a surely crucial factor but not an exhaustive description of a service virtue alone. A need for more eloquent and consistent ways to evaluate ambulance services seems to would attempt to truly innovate in the field of

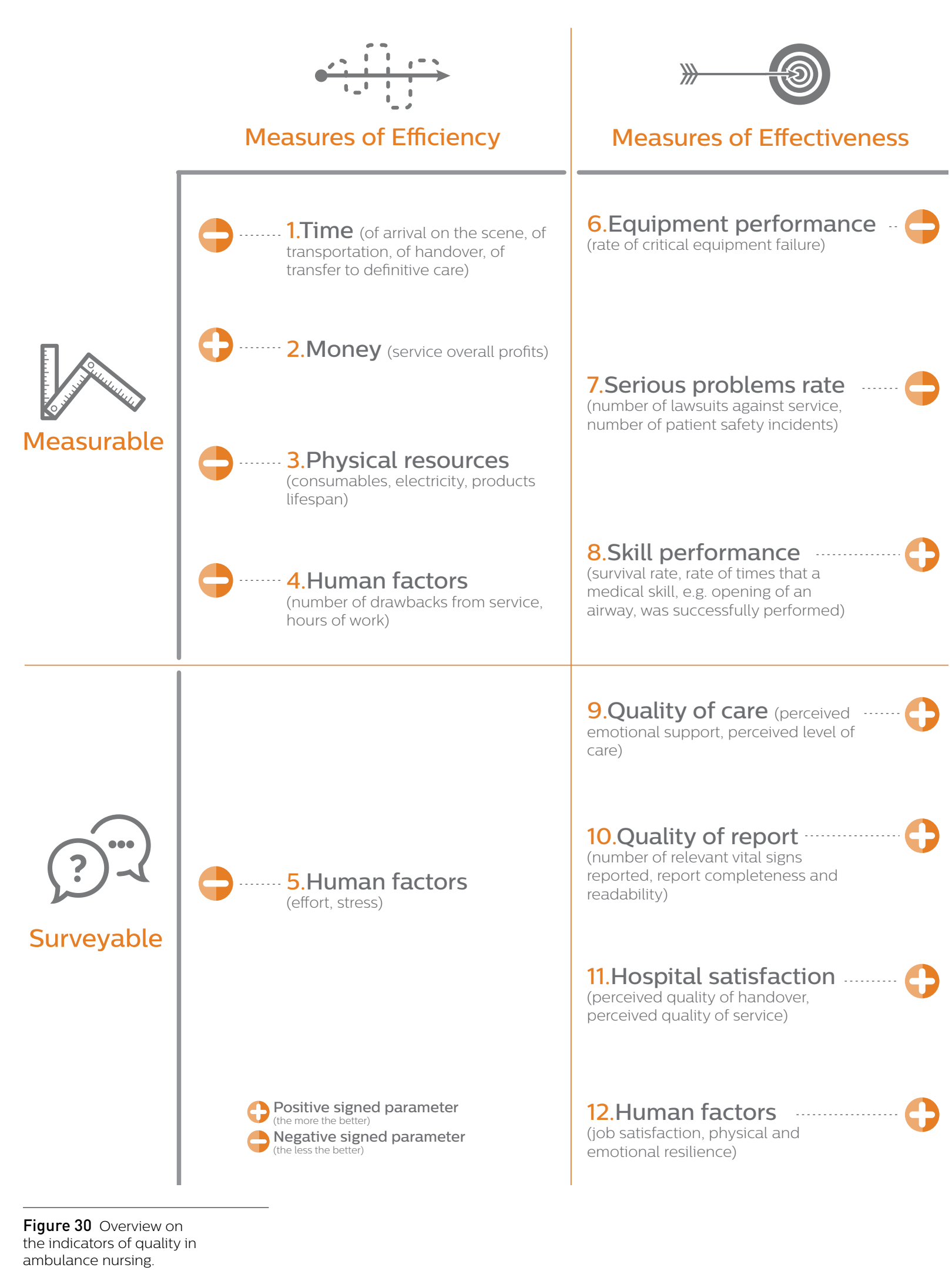

7




\section{Problem definition}

\author{
Implementing the analysis results
}

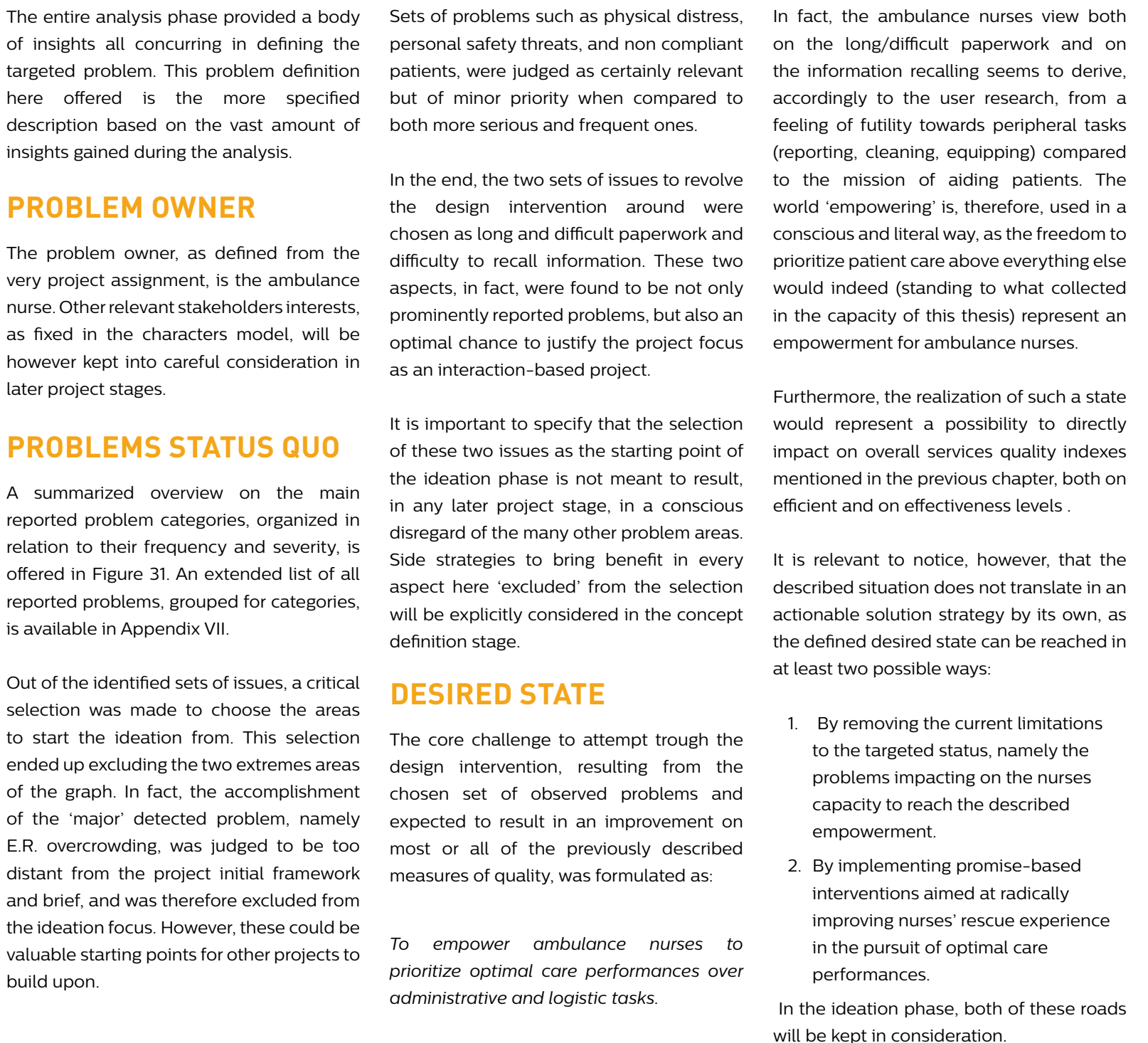

POSITIVE SIDE EFFECTS

Possible positive side effects connected to desired state

Improvement of the quality of patient care and of overall patient experience (measures of qually $7,0,9,11)$. The to primarily focus on patients could unlock their full 'care potential', and result in an improvement both on the medical and on the emotional support sides

Improvement of ambulance nurses overall salistaction at work (measures nurses to do what they like could bring to a greater professional fulfilment and to a decrease in workrelated stress.
Reduction of tasks completion time (measure of quality 1.). The opportunity to reduce the nurse efforts on 'unwanted' or 'disliked tasks may result in saving time otherwise spent on strugg

SIDE EFFECTS TO AVOID

\section{Possible side effects to avoid in the pursu}

of the described desired state are: The de-responsabilization of
ambulance nurses towards their administrative or logistic duties, causing possible adverse effects on (measures of quality 6 and 10 )
CONSTRAINTS

The main constraints in the pursuing of the desired state are recognized as

The need to fit the current EMS procedures

pressure staites to different time

environments

The need for healthcare providers validate the design

The willingness of ambulance nurses or other stakeholders to adapt to a

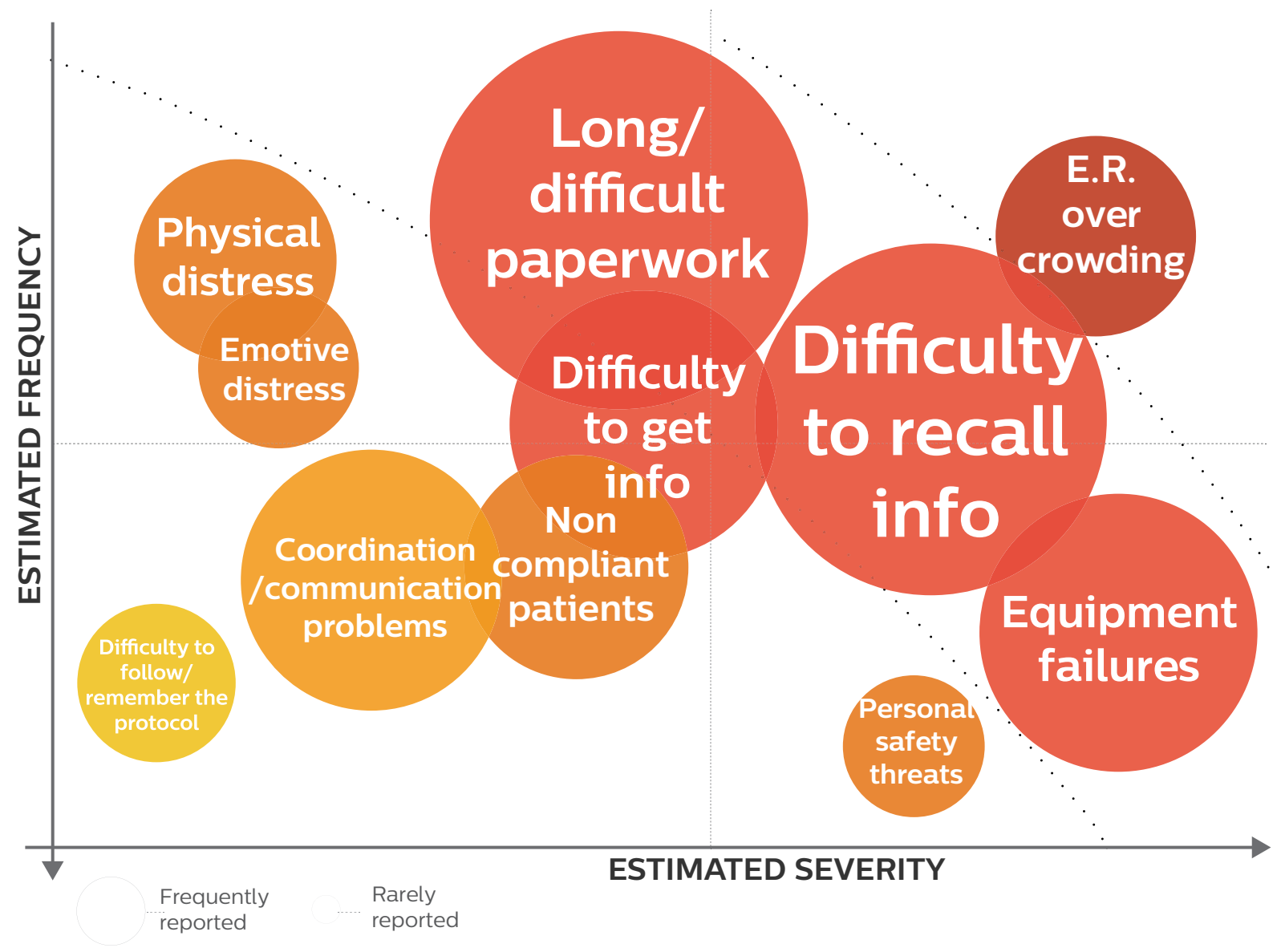

Figure 31 Problems 


\section{Scope}

Defining the design space

\begin{tabular}{|c|c|}
\hline $\begin{array}{l}\text { To specify what parameters are included into the } \\
\text { design and to outline the solution space, a scope } \\
\text { of the project was defined. Defining a scope was } \\
\text { assessed as a profitable inventory method to } \\
\text { spark inspiration towards ideation. }\end{array}$ & $\begin{array}{l}\text { The main desired effect of the intervention is } \\
\text { therefore formulated as providing an effortless } \\
\text { interaction on a product level, and facilitating } \\
\text { administrative tasks on a system level. On } \\
\text { the product level, the desired effect of the } \\
\text { intervention on the user will be to empower, } \\
\text { facilitate and speed-up the synchronic } \\
\text { work; while doing this, on the system level, } \\
\text { the diachronic work (information flow and } \\
\text { management) will benefit from being digitalized }\end{array}$ \\
\hline $\begin{array}{l}\text { The scope is visualized in Figure } 32 \text {. As can be } \\
\text { seen, the design will include one device and } \\
\text { a related digital environment, whose impact } \\
\text { is further specifed in the four quadrants. This } \\
\text { duality was decided in consideration of the } \\
\text { prominent importance data and communication } \\
\text { have in the field of EMS. Because of this } \\
\text { importance, a separate (digital) space in which } \\
\text { to manage information was assessed to be } \\
\text { possibly relevant to the topic. }\end{array}$ & $\begin{array}{l}\text { Accordingly, the situations in which to apply } \\
\text { the product intervention are defined to be } \\
\text { the ones in which this conflict between care } \\
\text { and administration is more evident, such as } \\
\text { the moments of collecting information from } \\
\text { the patient and the moments of reporting. } \\
\text { Still, the ambition of the system intervention is } \\
\text { larger scaled, as it is expected to have effects } \\
\text { throughout whole rescue chain. }\end{array}$ \\
\hline $\begin{array}{l}\text { As these two factors are in some moments } \\
\text { conflicting, the envisioned solution should } \\
\text { provide an easy, undemanding way to keep the } \\
\text { administrative part under control while focusing } \\
\text { on the patient. }\end{array}$ & $\begin{array}{l}\text { Under this point of view, it can be seen as } \\
\text { the indirect stakeholders of such a scoped } \\
\text { intervention are not only the ones actively } \\
\text { operating in the rescue team together with } \\
\text { ambulance nurses (drivers, physicians, } \\
\text { dispatchers), but also ambulance center } \\
\text { managers, GPs, and even researchers, insurance } \\
\text { and pharmaceutical companies, under the point } \\
\text { of view of the accessibility of structured rescue } \\
\text { data. }\end{array}$ \\
\hline
\end{tabular}

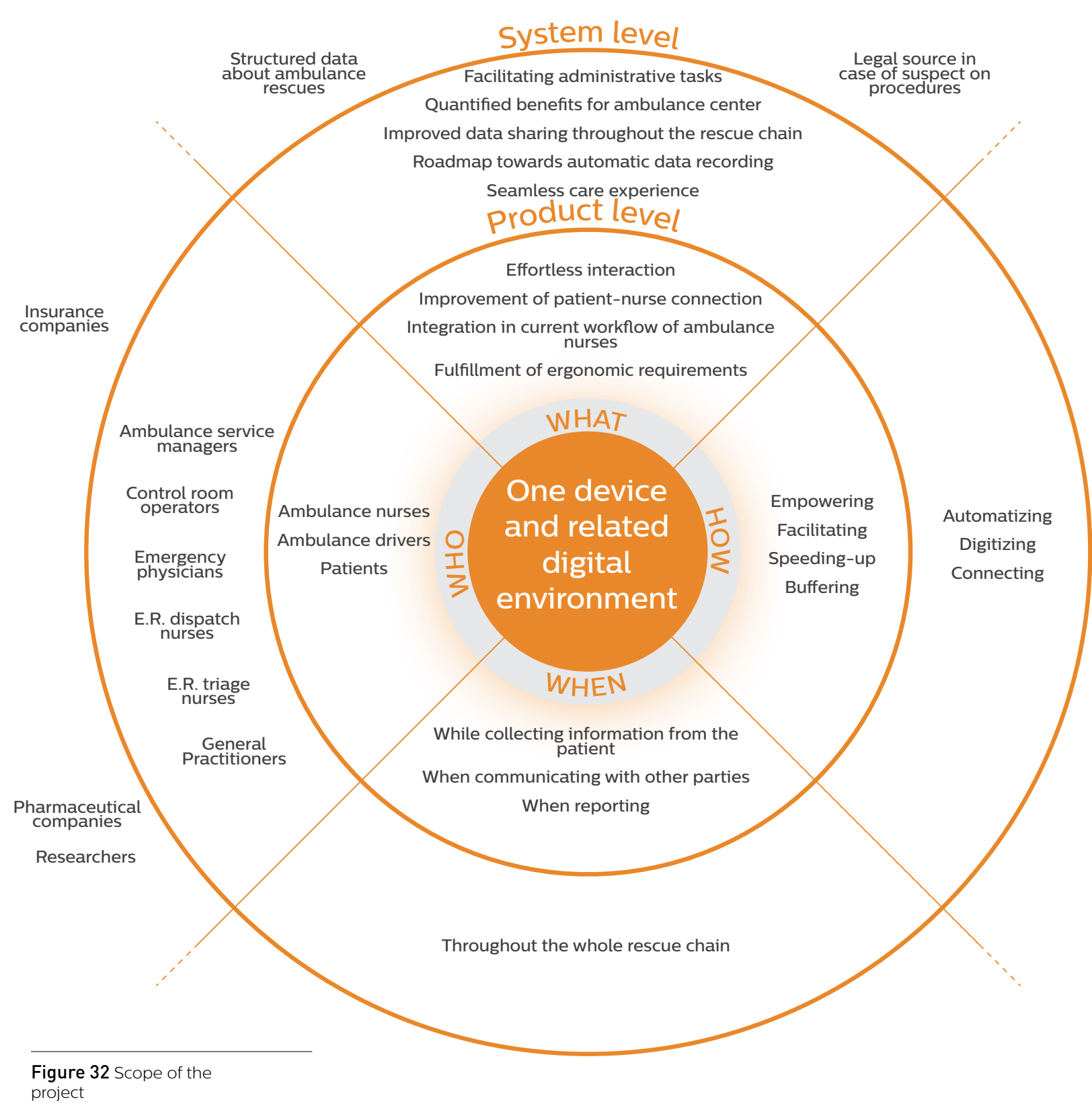




\section{Creative session}
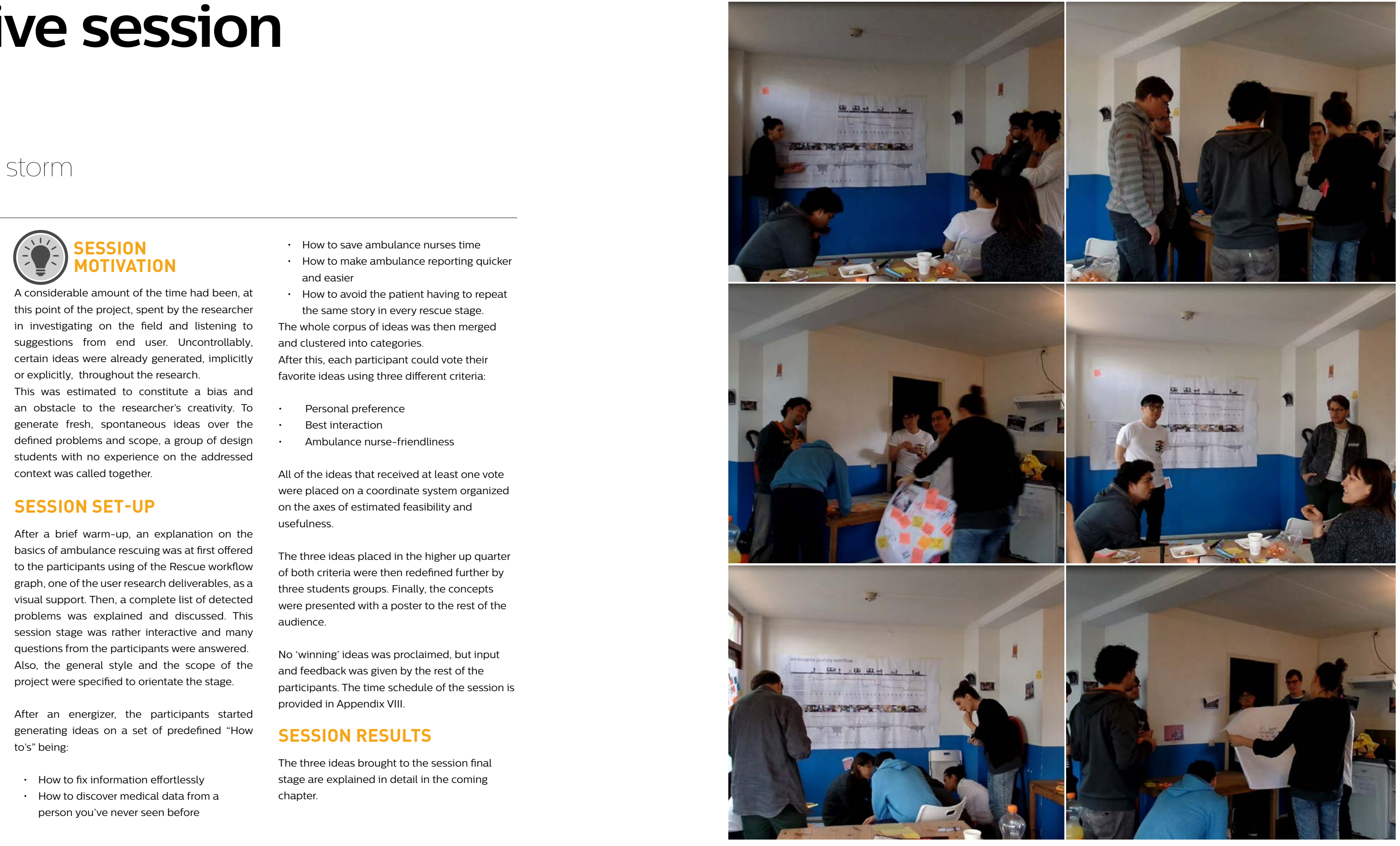


\section{Preliminary directions}

\section{Divergent solutions}

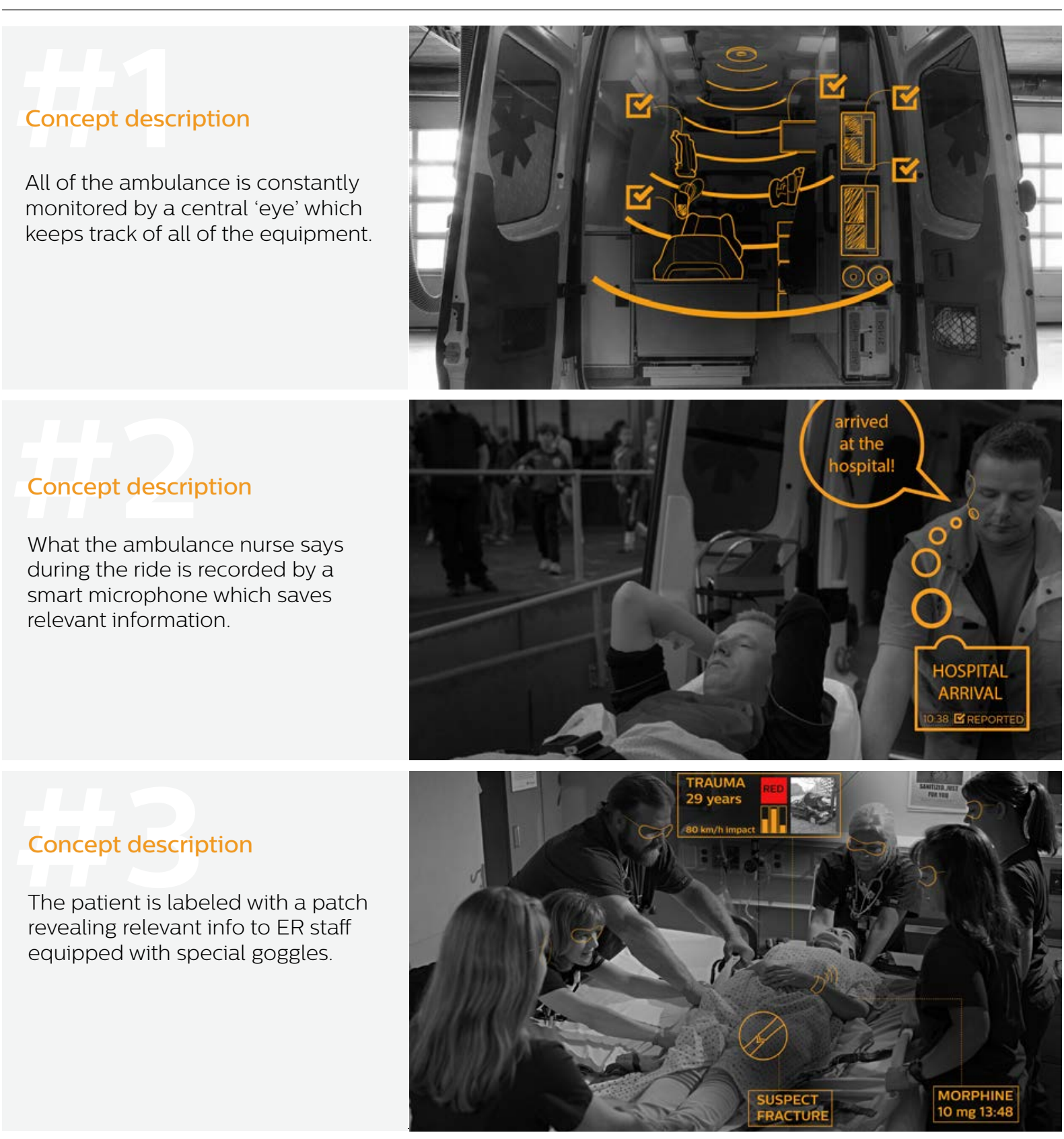

KEEP AN EYE ON THE EQUIPMENT

Advantages

Cross checks equipmen

before every shift

- Speeds up paperwork

- Permits to create long-

efficiency and patterns of use

Impact on workflow
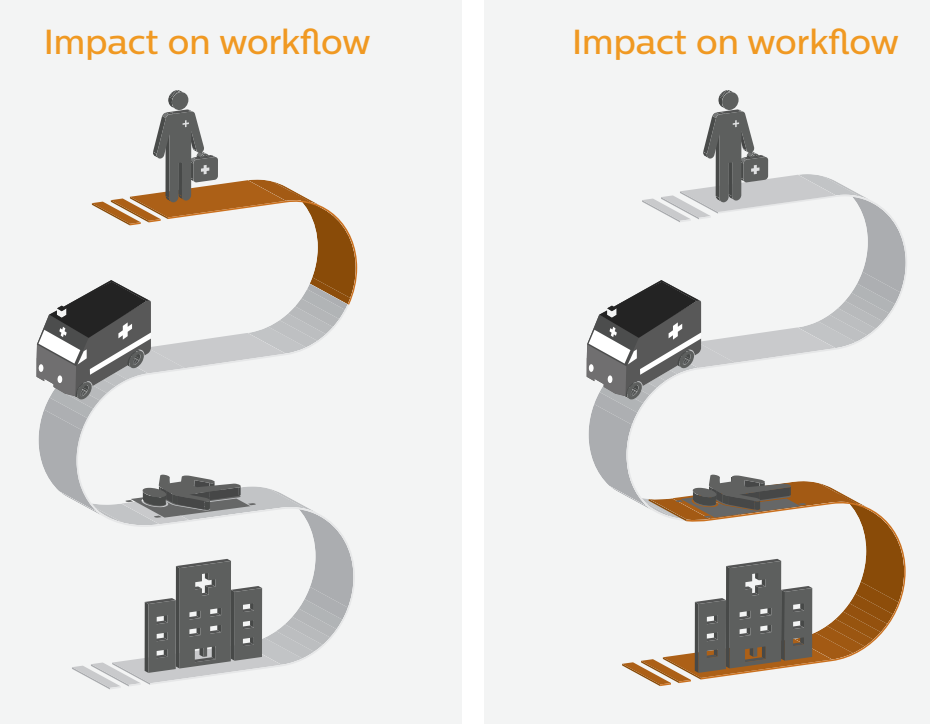

or writing on gloves

- Hands and eyes free

- Noise reduced

Faster reporting

Faster reporting

Quality measures addressed

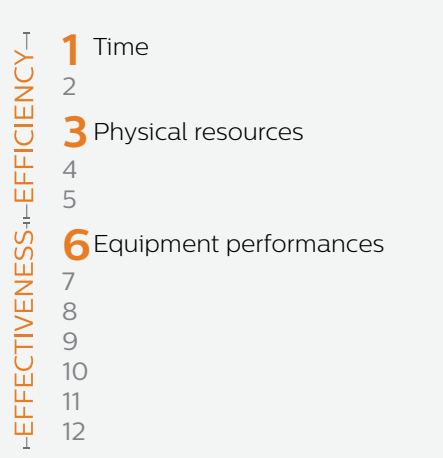

Expected nurses likability Straight-forward solution solving strong problem from user research,
not requiring big protocols changes.

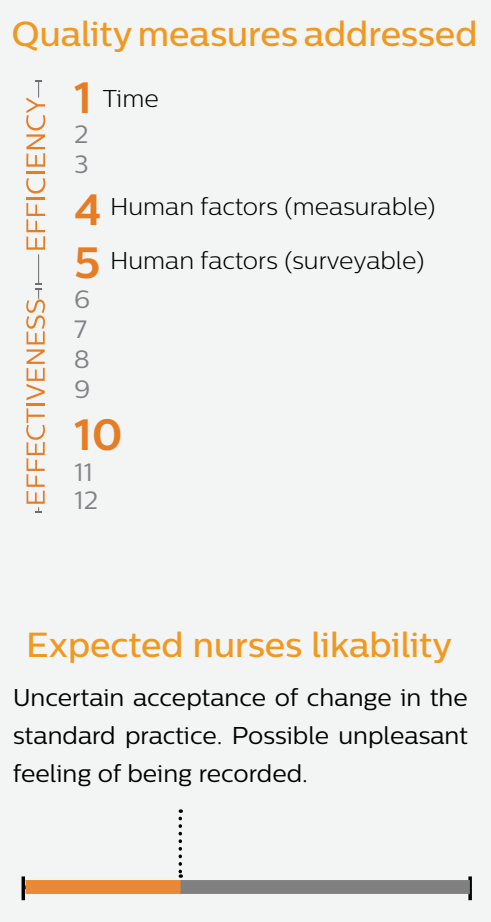

KNOW EVERYTHING IN ONE LOOK

Advantages

- Faster, more effective

handover

- Efficient patients

management in the SEH

- The patient privacy is safe

Impact on workflow

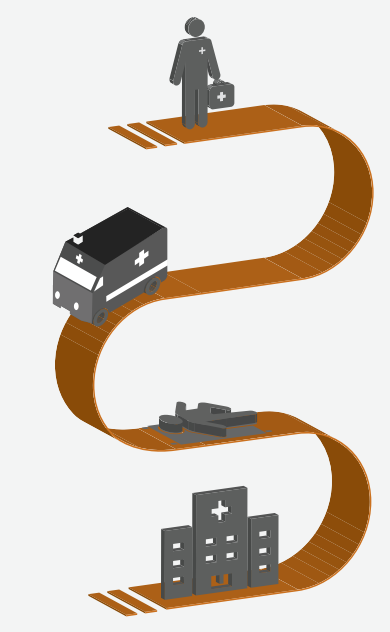

Quality measures addressed

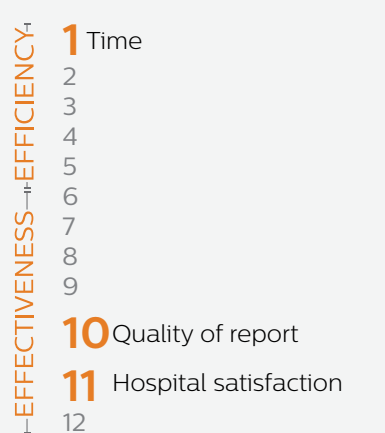

Expected nurses likability Large margin of uncertainty in fardirect benefits to ambulance nurses.

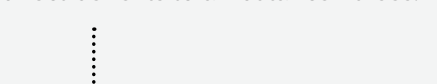




\section{Validation with users}

\section{(ㄱ)}

An exhibition displaying some of the main

research deliverables and demonstrating posters

garage of a big ambulance center in den Haag

for the duration of two shifts. Workers going and coming from rescue could stop by, observe, and
informally give their view on the three directions.

MAIN RESULTS

The reactions from the nurses were partly aligned to the expectations, partly surprising. As expected, the first direction caught the attention and the favor of a vast number of attendants.

Not as predictably, the second option got very warm reactions and triggered interesting possible side uses of the concept (particulatys)

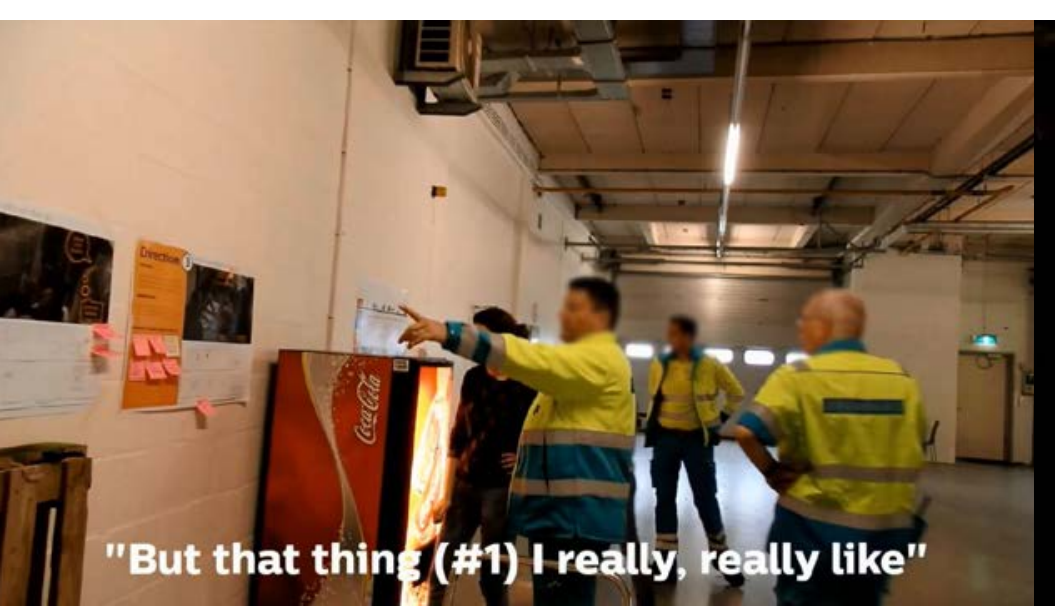

The third direction, conversely, was understood
but overall not preferred by a large number but overall not preferred by a large number of it as their favorite one). A summarizing video of the live reactions of the nurses to the three directions is available at the link https://vimeo this password Effortlesslinteractions.

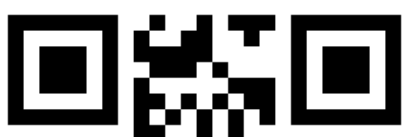 rytras

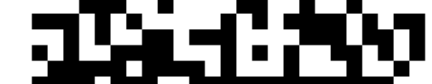 回部要}

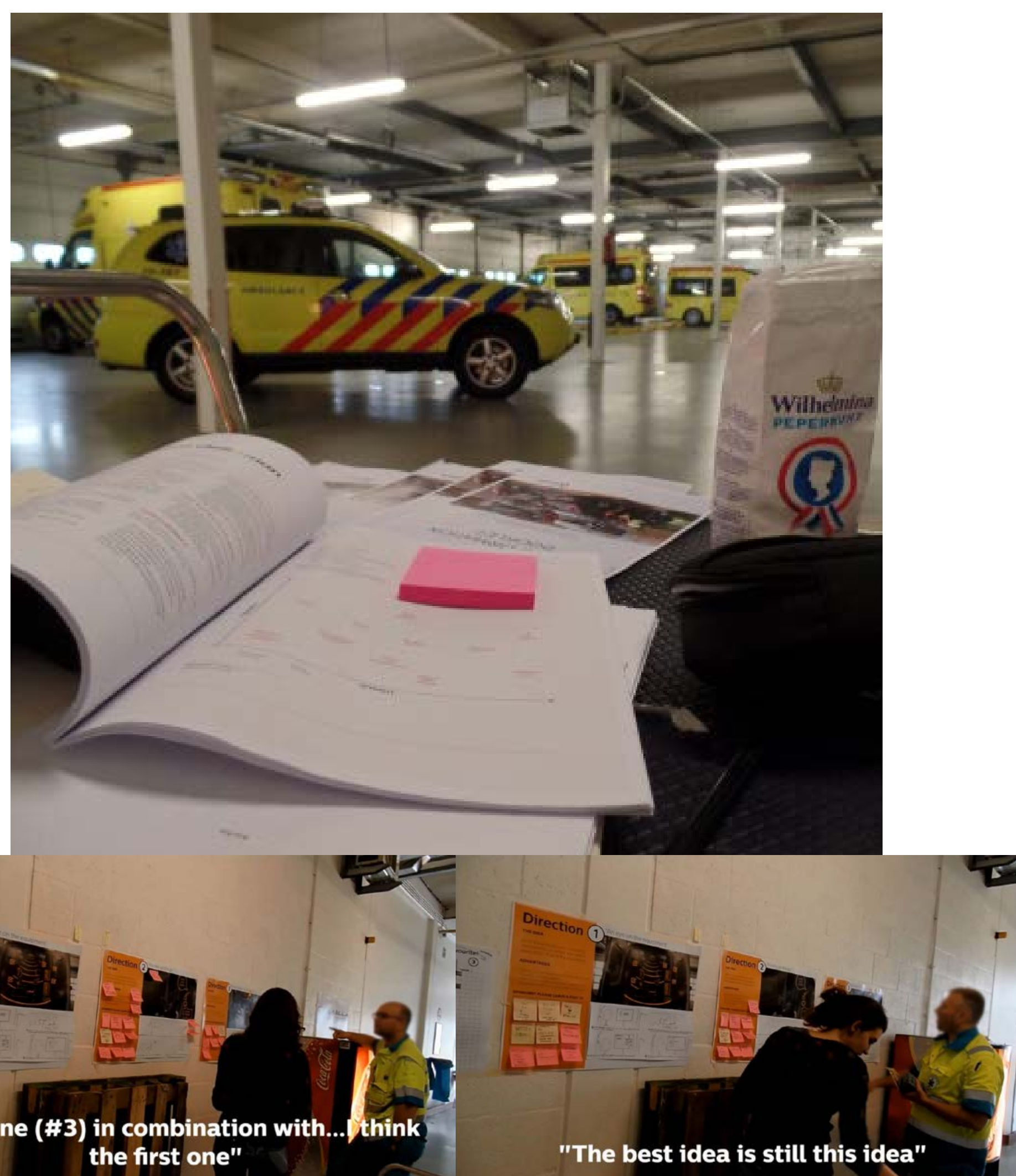

"I think it's both nice (\#2 and \#3)"

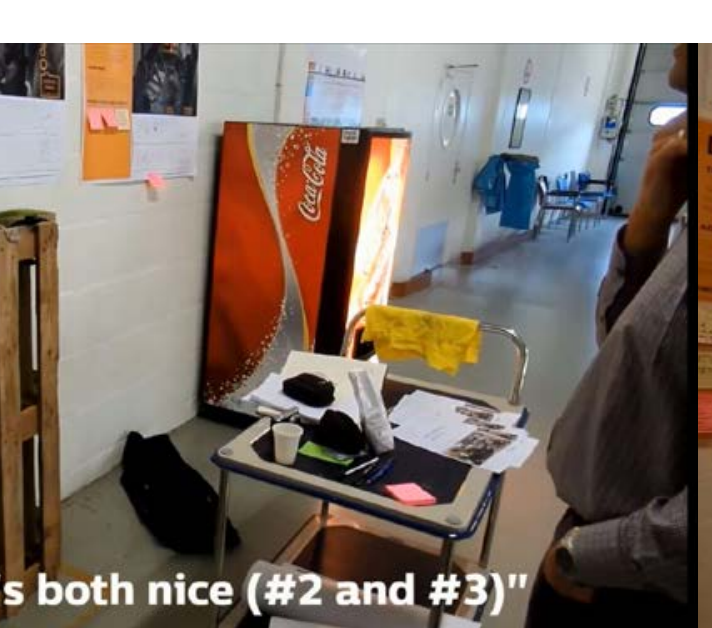

"That one (\#3) in combination
the first one"

Figure 34 Participants
opinions and preferences 


\section{Direction choosing}

Selecting the most promising option
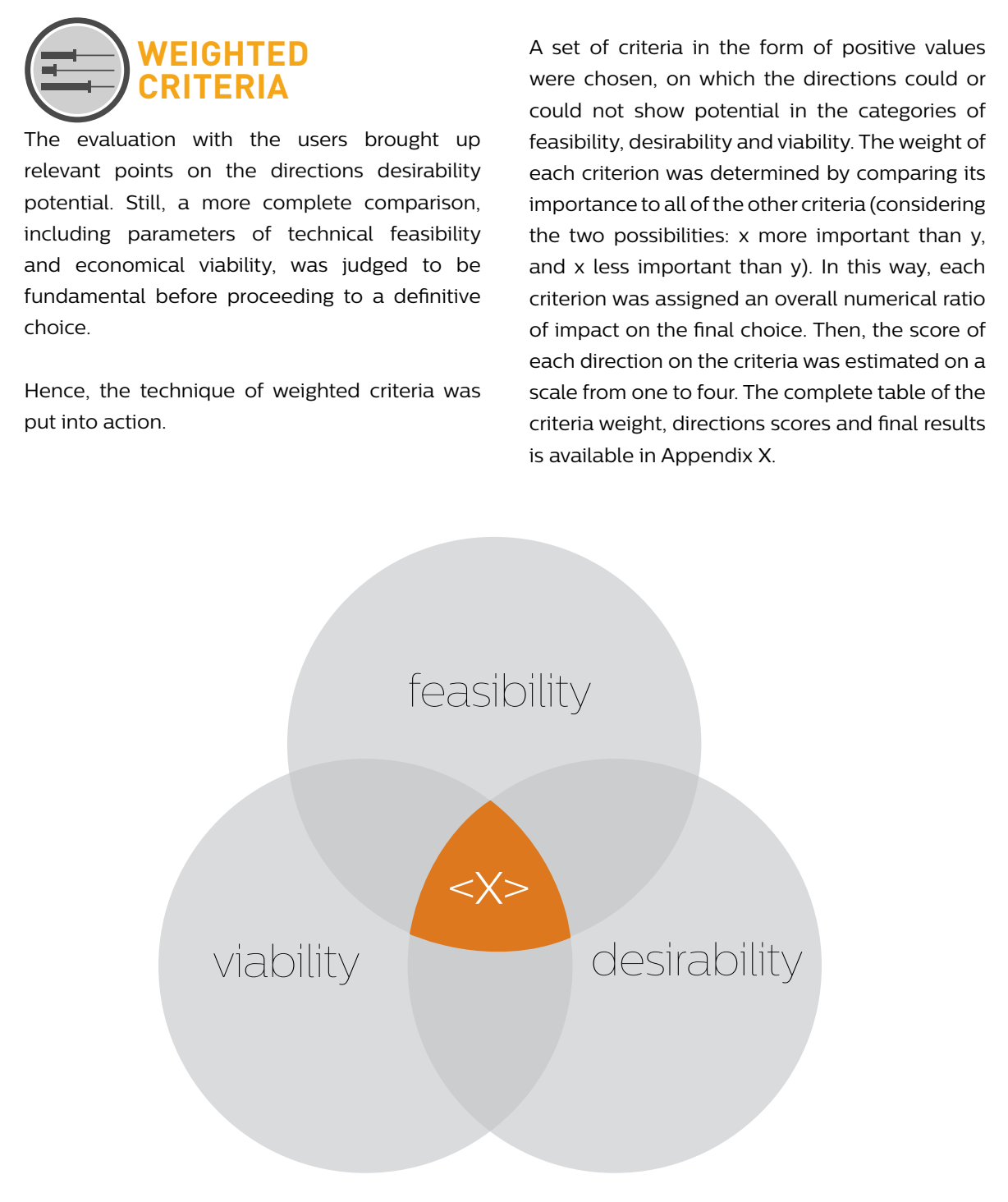

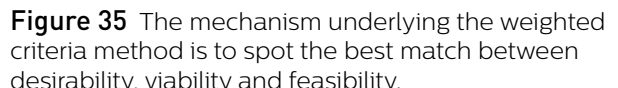

CHOSEN DIRECTION

The weighted criteria method brought to a decisive victory of the second direction, followed respectively by the thed and the inst one. This the users during the validation, as shown by the senerally warm wetcome reserved to the solution envisioned in direction two.

Conversely, a strong discrepancy is observable etween the results of the weighted criteria and the enthuslasm shown by the ambulance nurses for the first direction. Overall, it is relevant to notice that the absolute value of equipments beneft in ambulance nursing

Still, this direction scored relatively low in several criteria judged as important for the present project. From the point of view of economical viability, in particular, this direction was evaluated to be not particularly cony terms of fit with the Philips brand

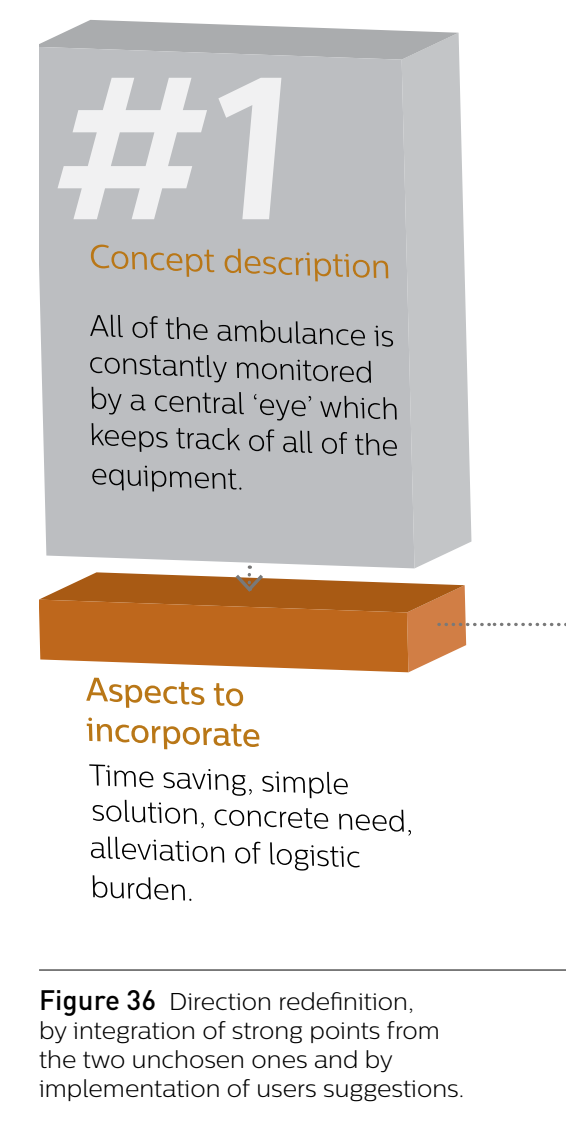

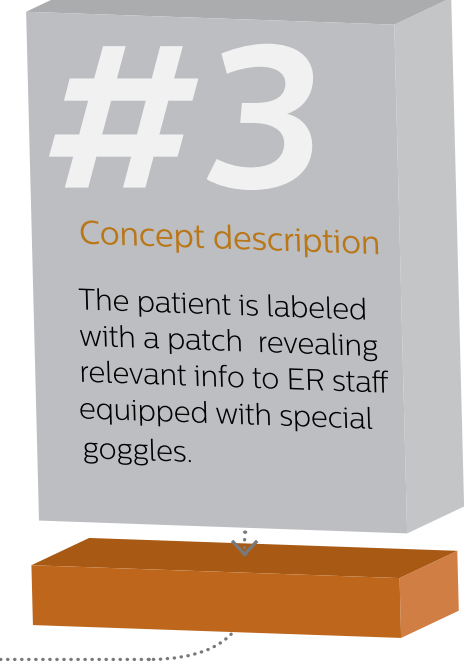

Aspects to incorporate Easier data transfer agging, possibility to $\begin{array}{ll}\text { Aspects to enhance } & \text { save more and richer } \\ \text { data from the scene }\end{array}$

decisional aid,

training and research plications.

Nevertheless, different companies or institutions the other two possibilities were chosen not to be dislegarded. The motivation of the positive abandoned directions were broke down into could realistcally be int, have value pornts hit

Also, suggestions and ideas from the users about ways to maximize the impact of direction
number two were selected and fixed, to be taken ins consideration for further detailing

An overview on this
available in Figure 36 . 


\section{Future vision}

Developing a meaning

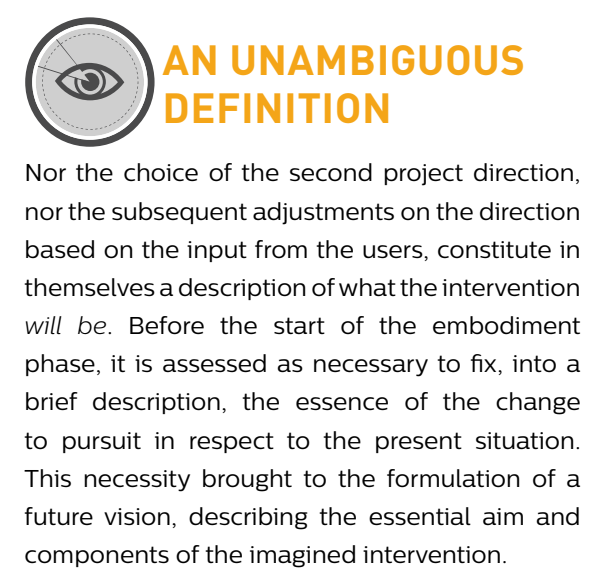

THE ENVISIONED IMPACT

The primary desired effect of the intervention, and its reason to exist, is defined as the empowerment of the ambulance nurse to
leserve his or her complete focus to the care of the patient from the whole duration of the rescue.

This empowerment is envisioned to be obtaine through a relief from the burden of reporting quality of rescue reports.
A condition to the realization of this vision is thus, the implementation of a system enabling the reporting responsibilities while assisting the

Fom the user research phase, the kind of burden impacting on the nurses capacity to te dentified as the need to fix data to be successively eported. Therefore, the envisioned solution is imagned to include a physical component is of patient care Specifcally it is envisioned for this physical support the characteristic of bein operable hands- and eyes-free

In order to saturate and enhance the advantages of such a physical component, it is envisioned for the intervention to include a digital environmen in which the inputs from the device (in the form of unstructured, real-time data) are organized and accessible information.
FROM A DESIRED STATE TO A FUTURE VISION

It is relevant to take note of the narrowing in the project focus obtained with the passage from problem definition chanter) to the more specific future vision here described

While in the desired state the ultimate effect of patient care prontizing was already stated, the way to reach tils effect was left open. In the future vision, conversely, a rather specific description or how tor

Precisely in this difference lies the work of refinement of ideas and meanings which constituted the core of the conceptualization section. From the formulation of the future vision, it is possible to proceed into operative decisions regarding the system embodiment.

A system that empowers ambulance nurses to prioritize patient care by permitting seamless fulfilling of the administrative responsibility. The system is composed by a device, permitting continuous, effortless input of administrative data, and a digital environment organizing and structuring the inputs from the device.
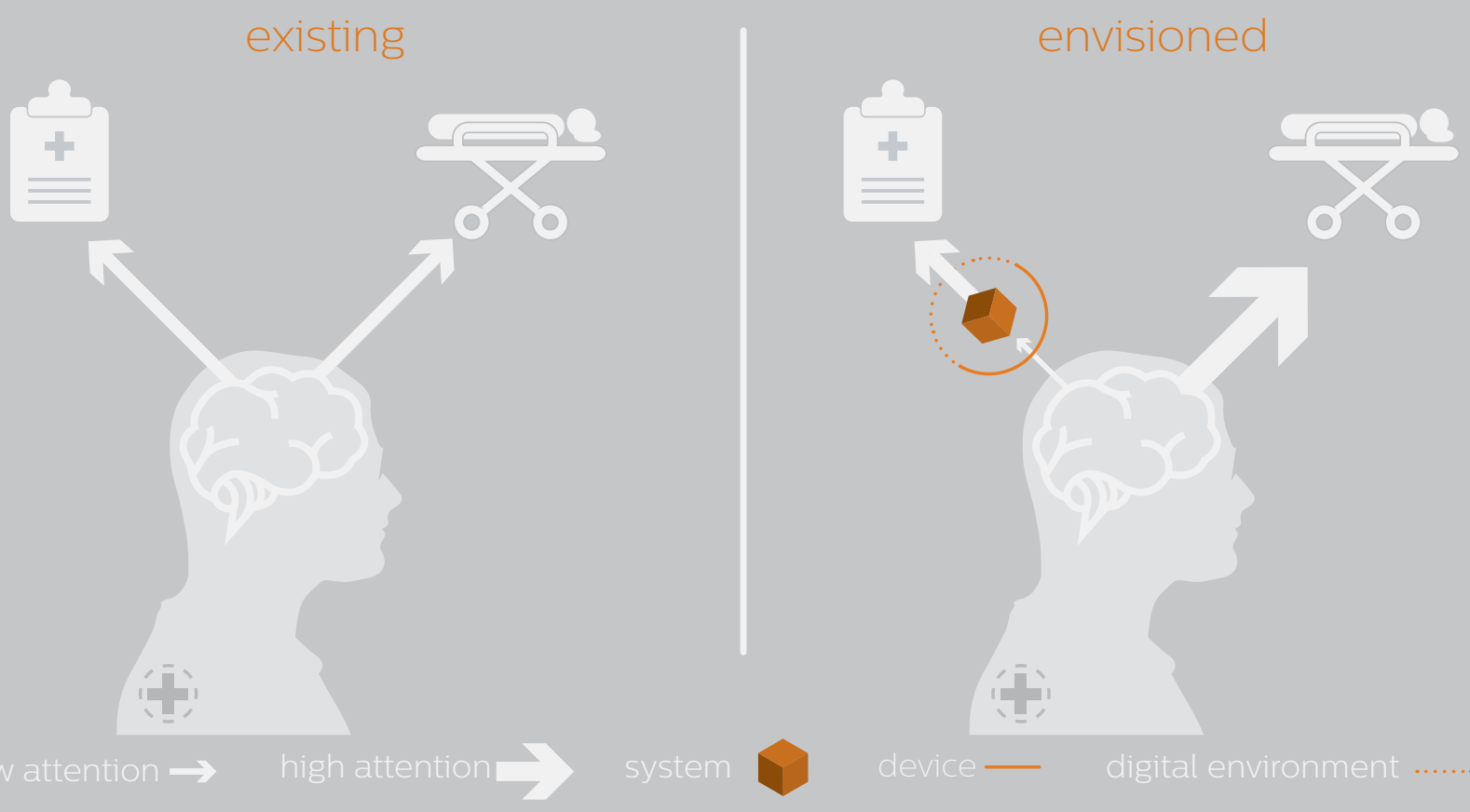


\section{Conceptualization}

methods

(a) SCOPE

To specify and define the solution space of the design intervention, a scope of the
project, including the planned impact of both the imagined product and system was project, including the planned impact of both the imagined product and system, was
defined.

\section{(1)}

To gather fresh, unbiased ideas on the design problem, a creative sessions with
several design students was organized. The session brought to the generation of three
design directions.

\section{VALIDATION WITH USERS}

To better assess the potential desirability of the three design directions, they were
presented to end users who were given the chance to vote for their favorite one.

WEIGHTED CRITERIA
In addition, a set of criteria was developed to assess the overall advantages of each
direction. Together with the validation with the users, this method brought to the
selection of one design direction to follow.

FUTURE VISION
The selected direction was then redefined, and its core meaning clarified by creating
a future vision able to describe the desired effect of the intervention on the overall
practice of ambulance nursing. 


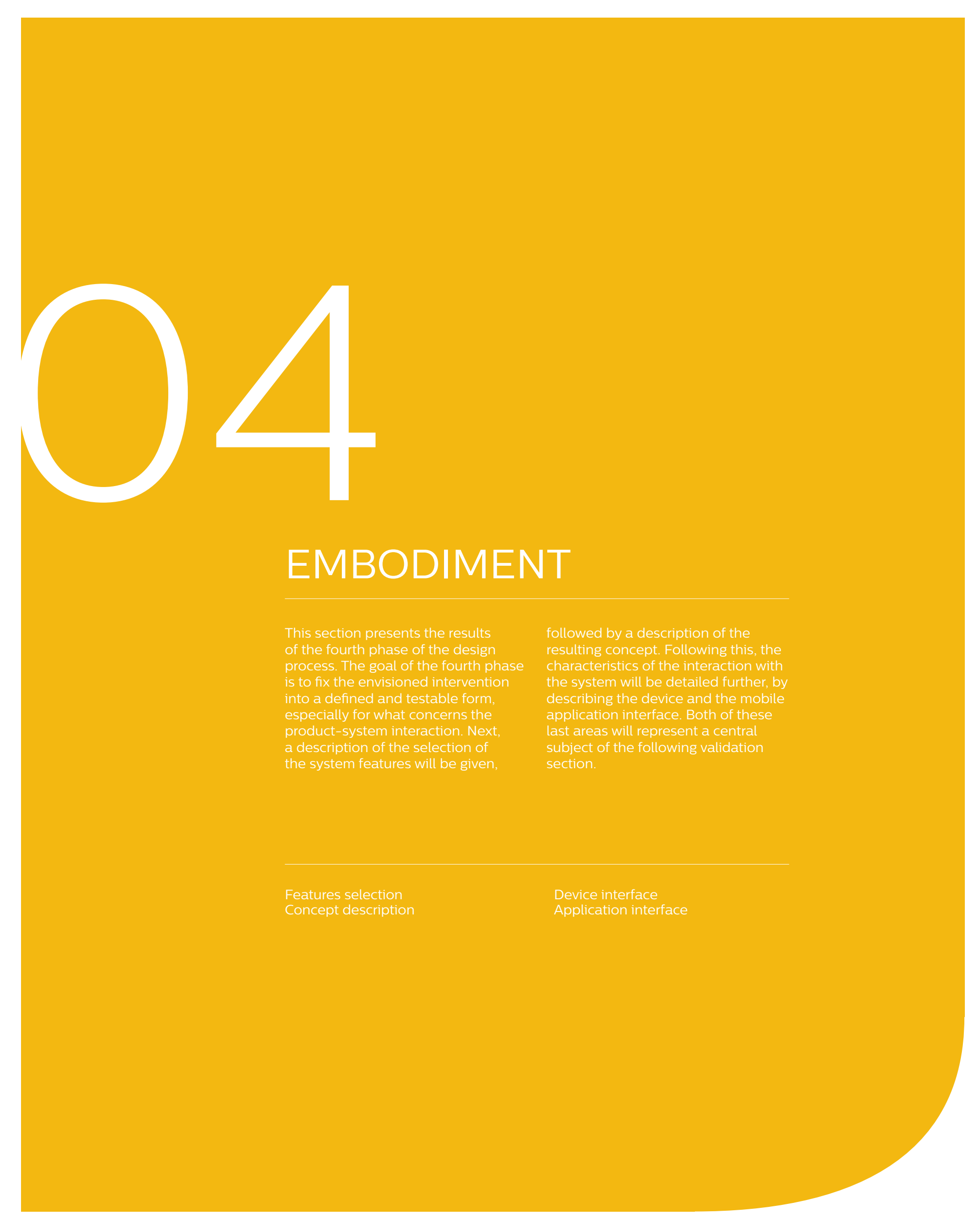




\section{Features selection}

\author{
Materializing the empowerment
}

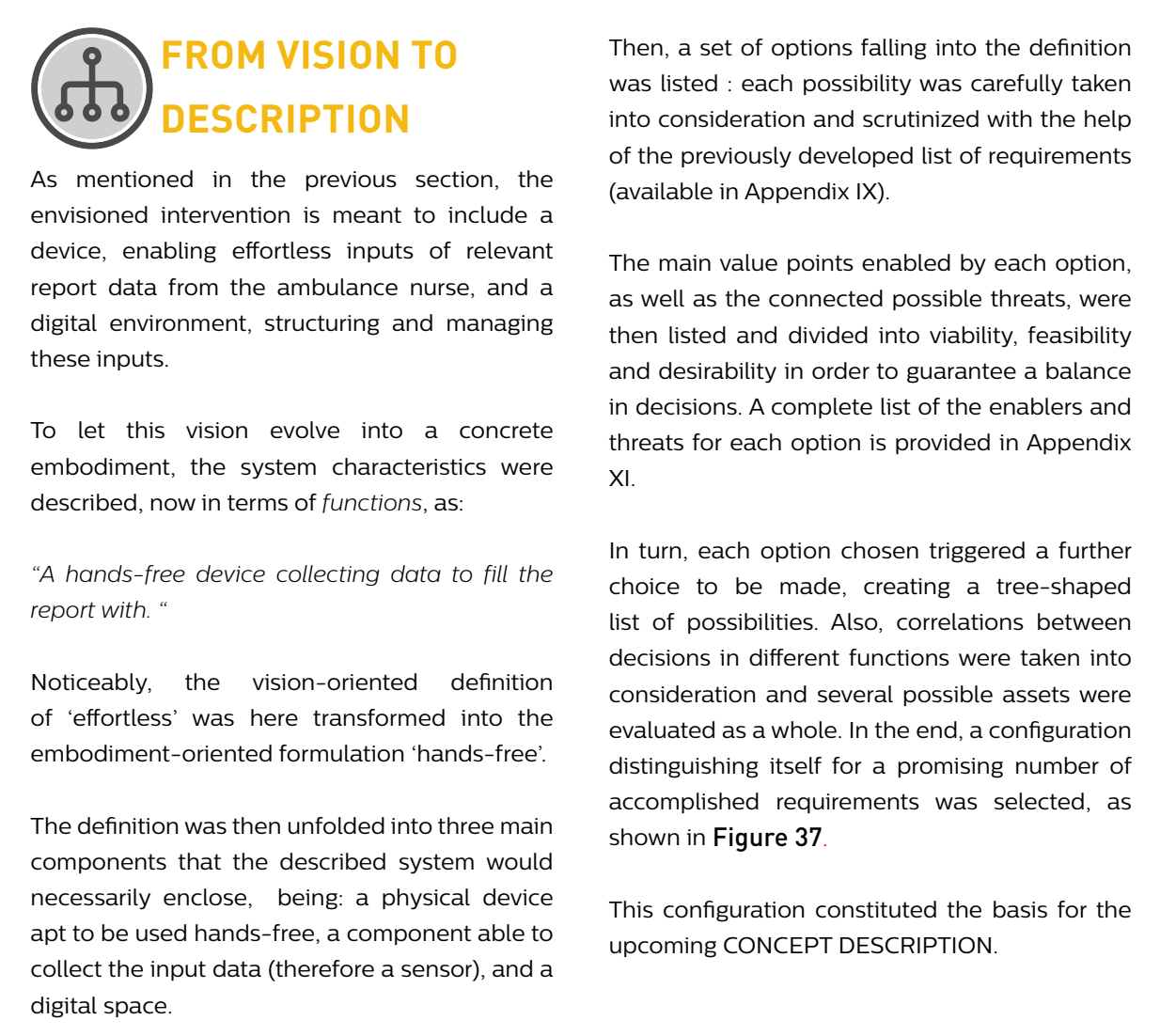

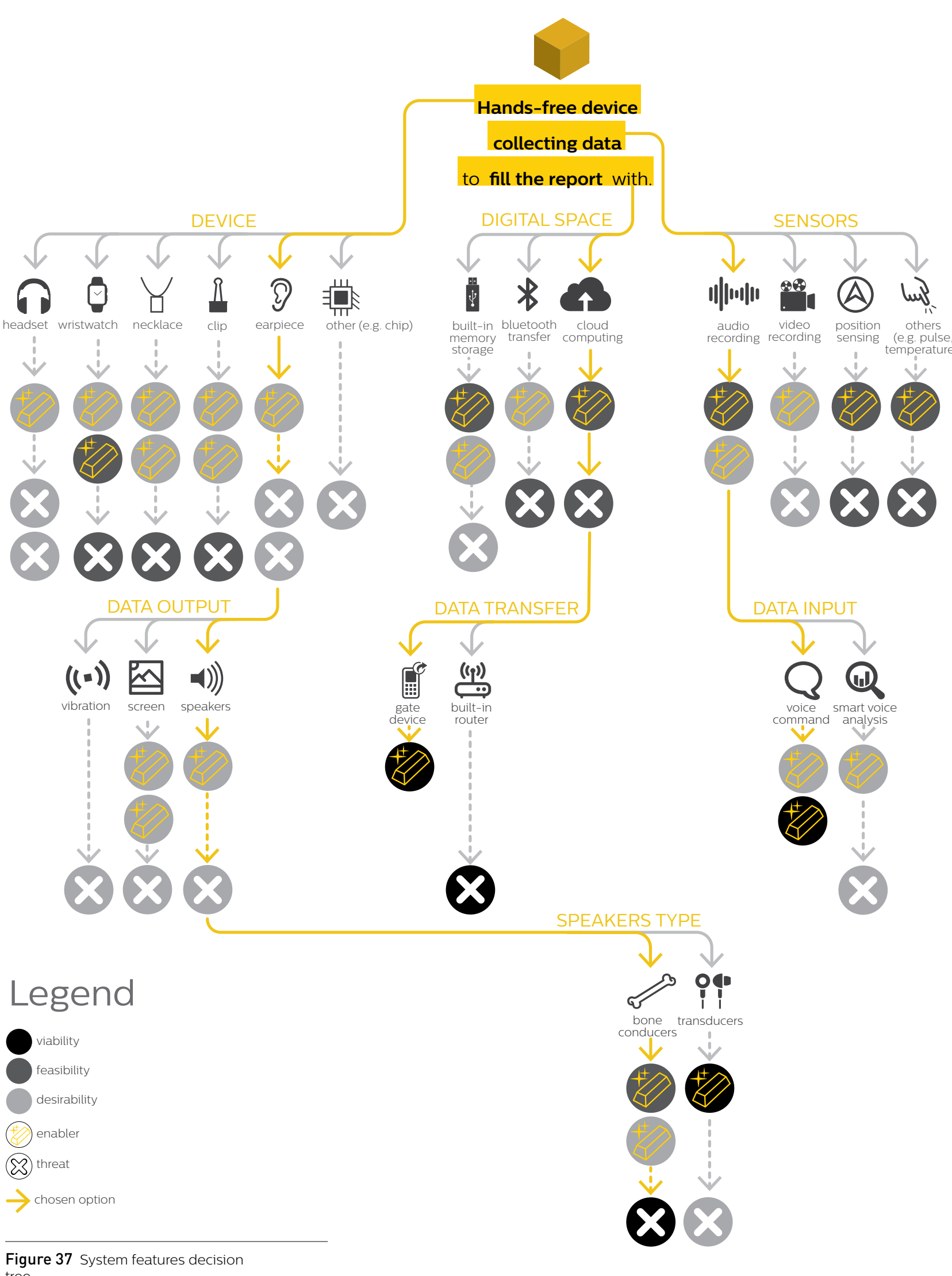




\section{Concept description}

\section{What does the product do?}

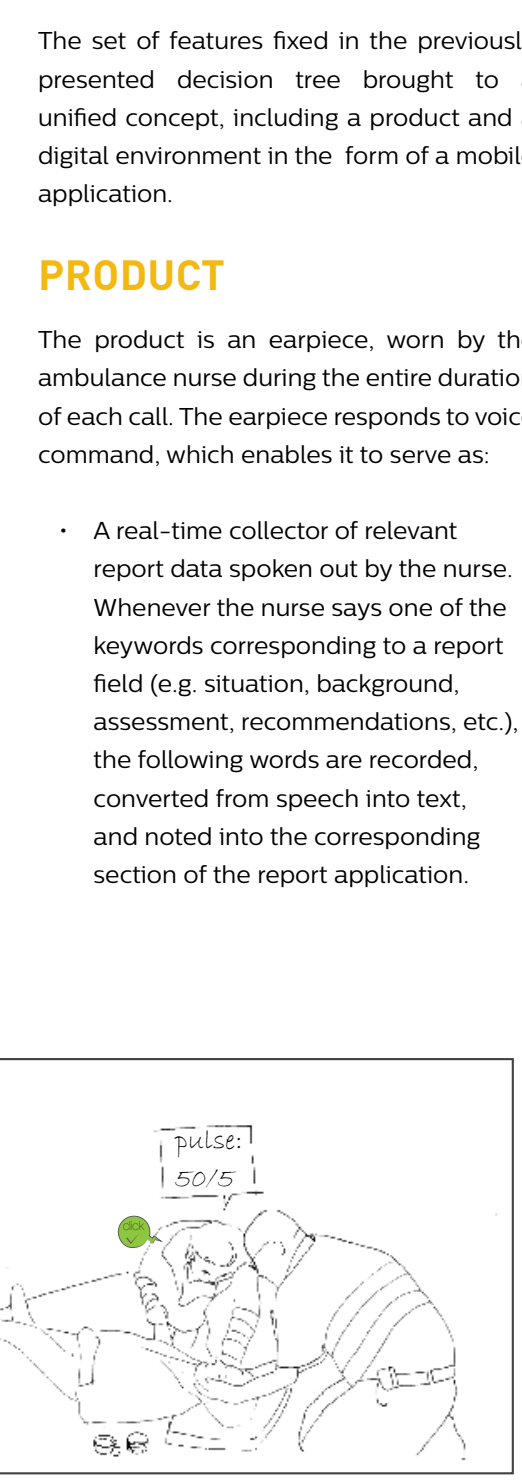

Data relevant to the report are captured from nurses voice inputs.

\section{For instance, would the nurses say:} prompted to fill with the value ' 50 ', space corresponding to 'pulse' in the report application.

Ahands-free interface for telephone calls, as the nurse can both respond to and initiate calls by voice

A hands-free cor

Ahands-free controller for the would the nurse say the keywords 'Activate screen' followed by a medical protocol name (e. COPD) the monitor will be prompted to display the decisional algorithm corresponding to the official protoco regarding COPD cases

MOBILE APPLICATION

The digital environment connected to the physical product inputs is accessible by mean of a digital application compatible
with smartphones, tablets and laptops.

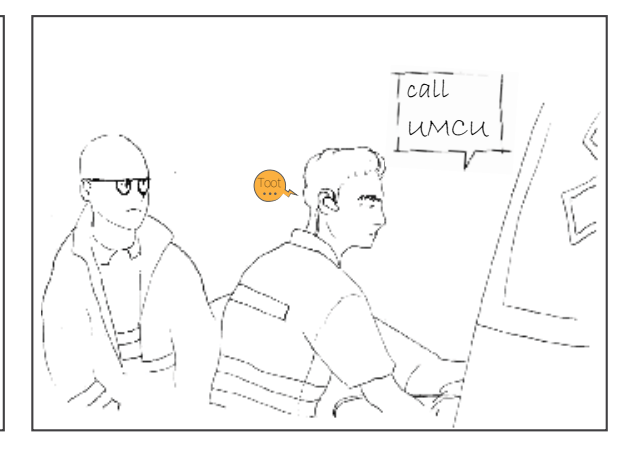

Phone and radio communications are funnelled in the earplug.
The application's primary use is administrative reporting. After the ride, the already filled in with the inputs collected by the physical product. The task of the nurse is, therefore, reduced to a check of the accuracy and completeness of the collected information

Furthermore, other potential users of the hospitat and the control ro destination the possibility to consult in real time the emergency case development.

NAME

The concept was given the name of Philips LiveSync. This was decided in effortlessly fix onging case details in ral time metaphorically synchronizing the ambulance nurse situational awareness with the rest of the distributed team.

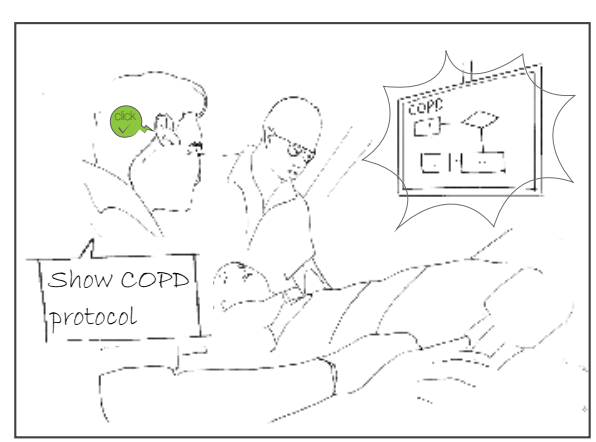

Reminders and information are shown in the ambulance monitor.

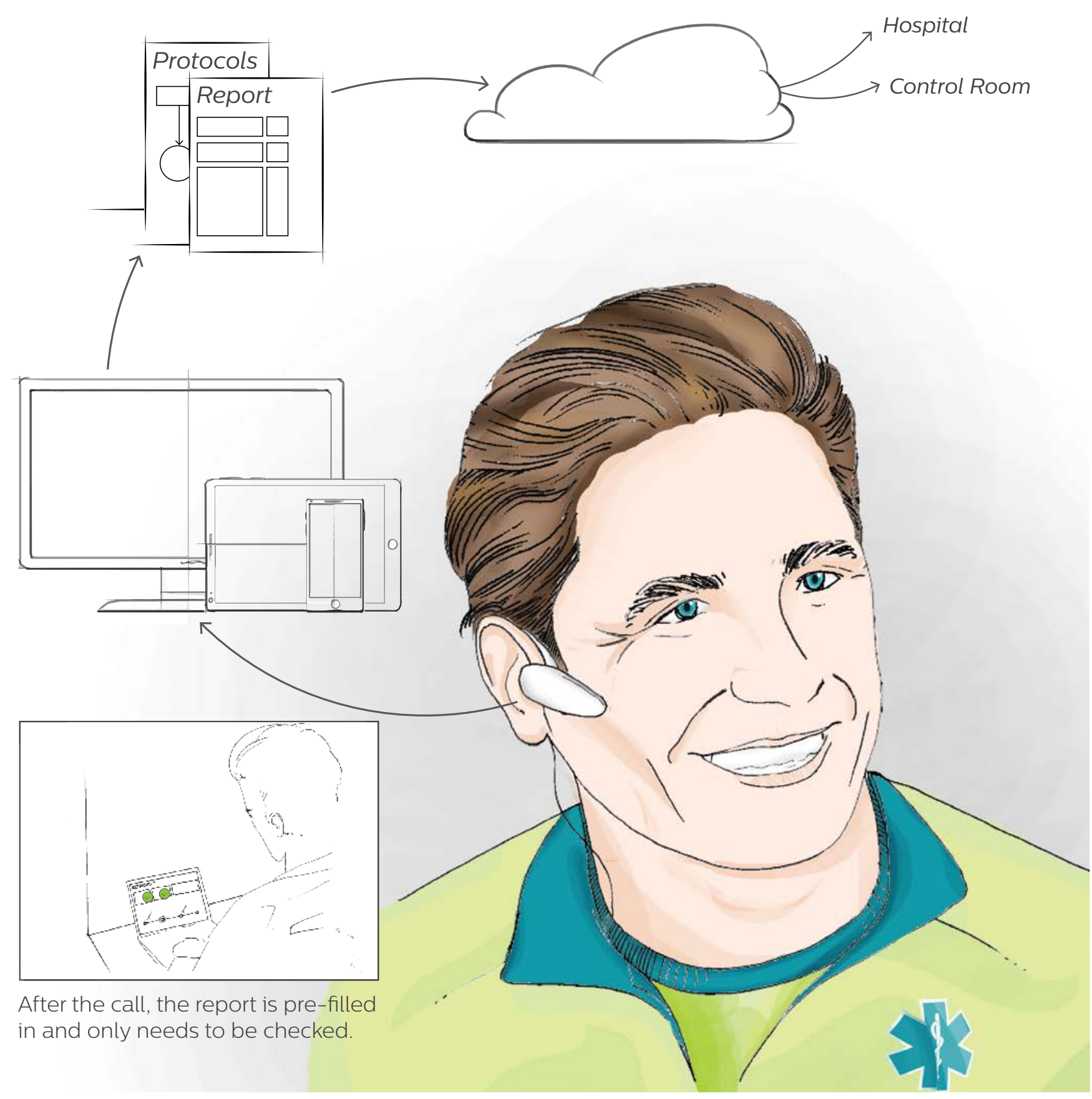




\section{Device interface}

Enabling the flow

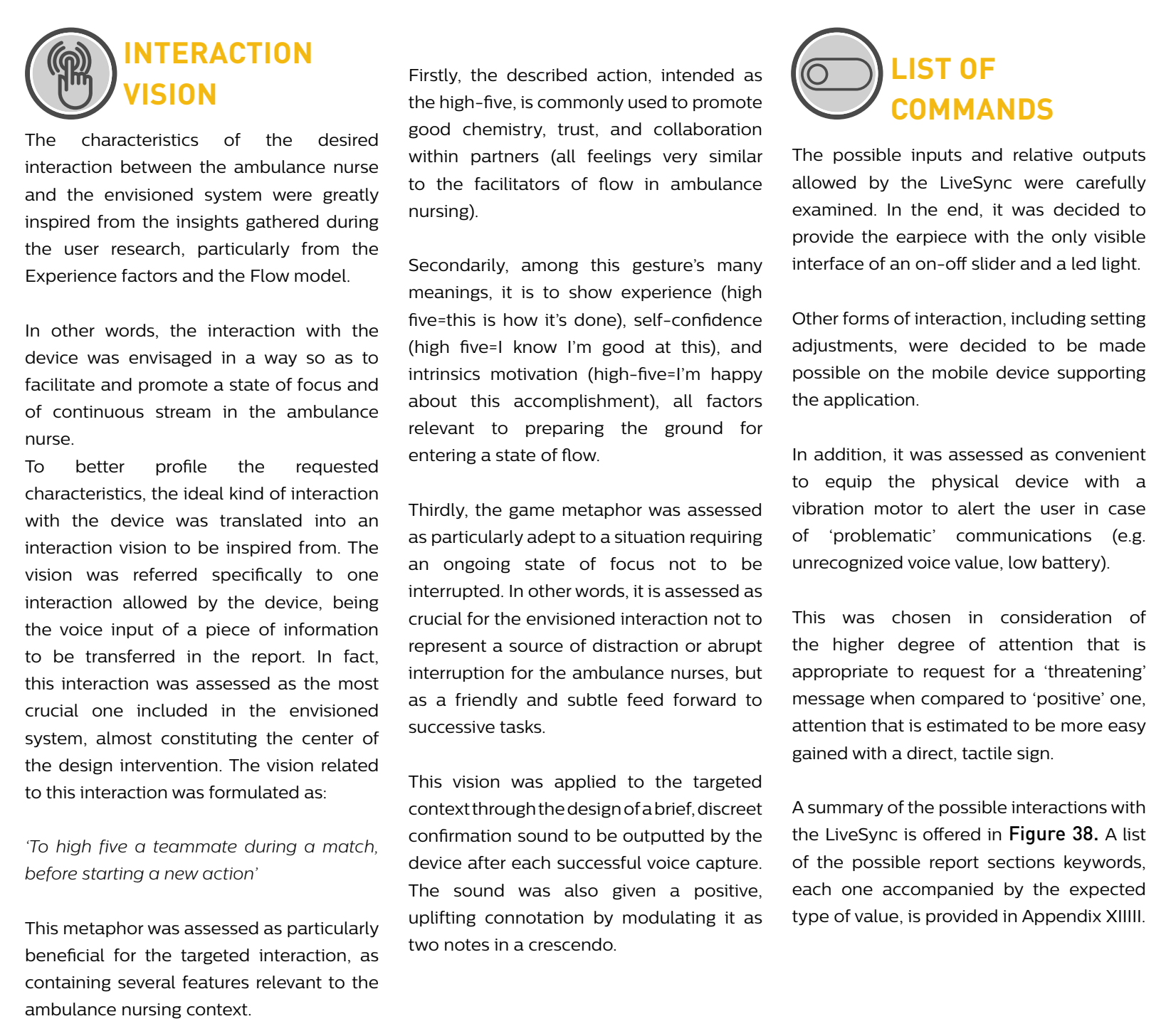

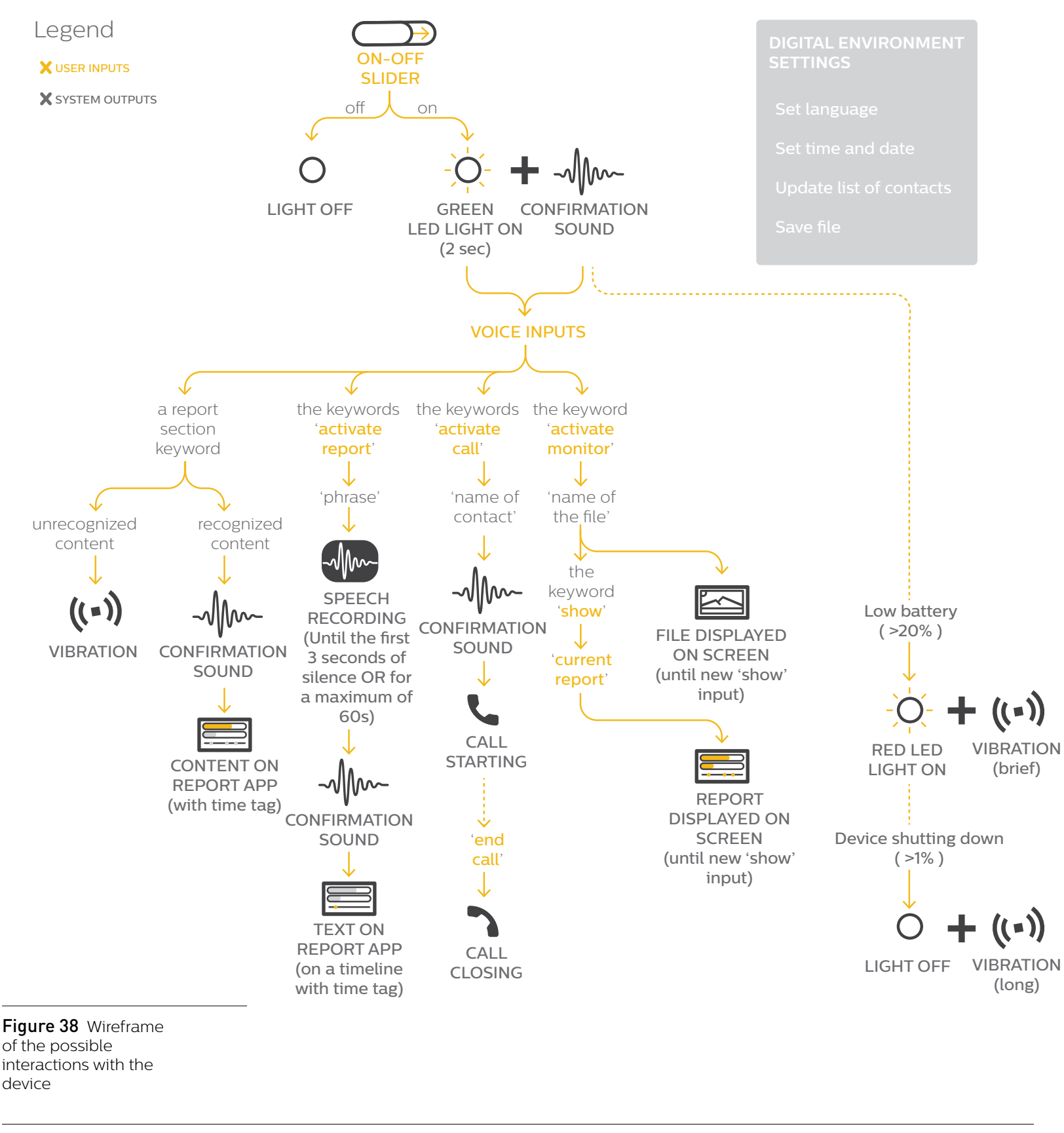




\section{Application interface}

Making administration easier

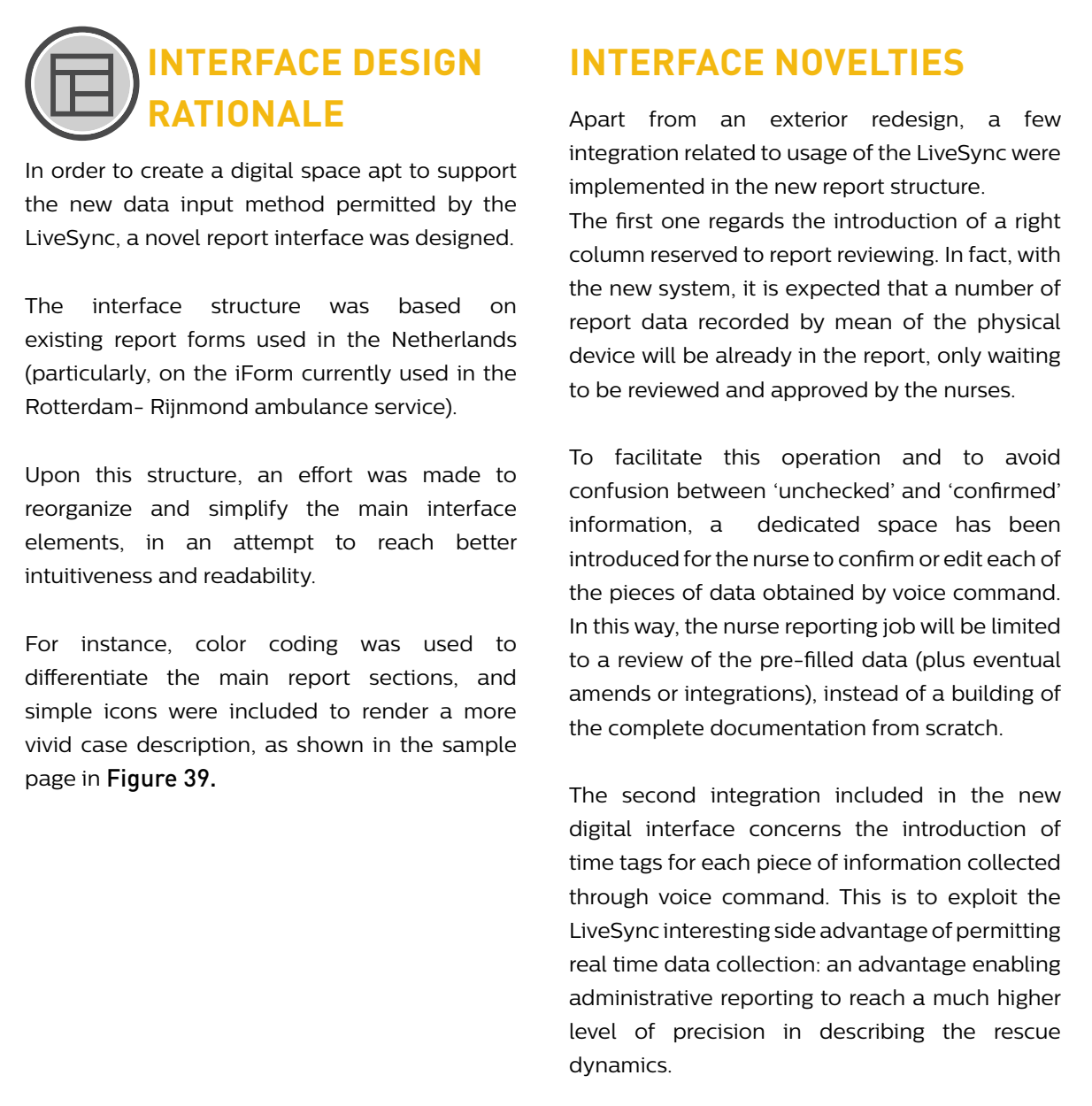

Airway

Circulation

Disability

Exposure

Primary Survey

Confirm

Report review
Report sections

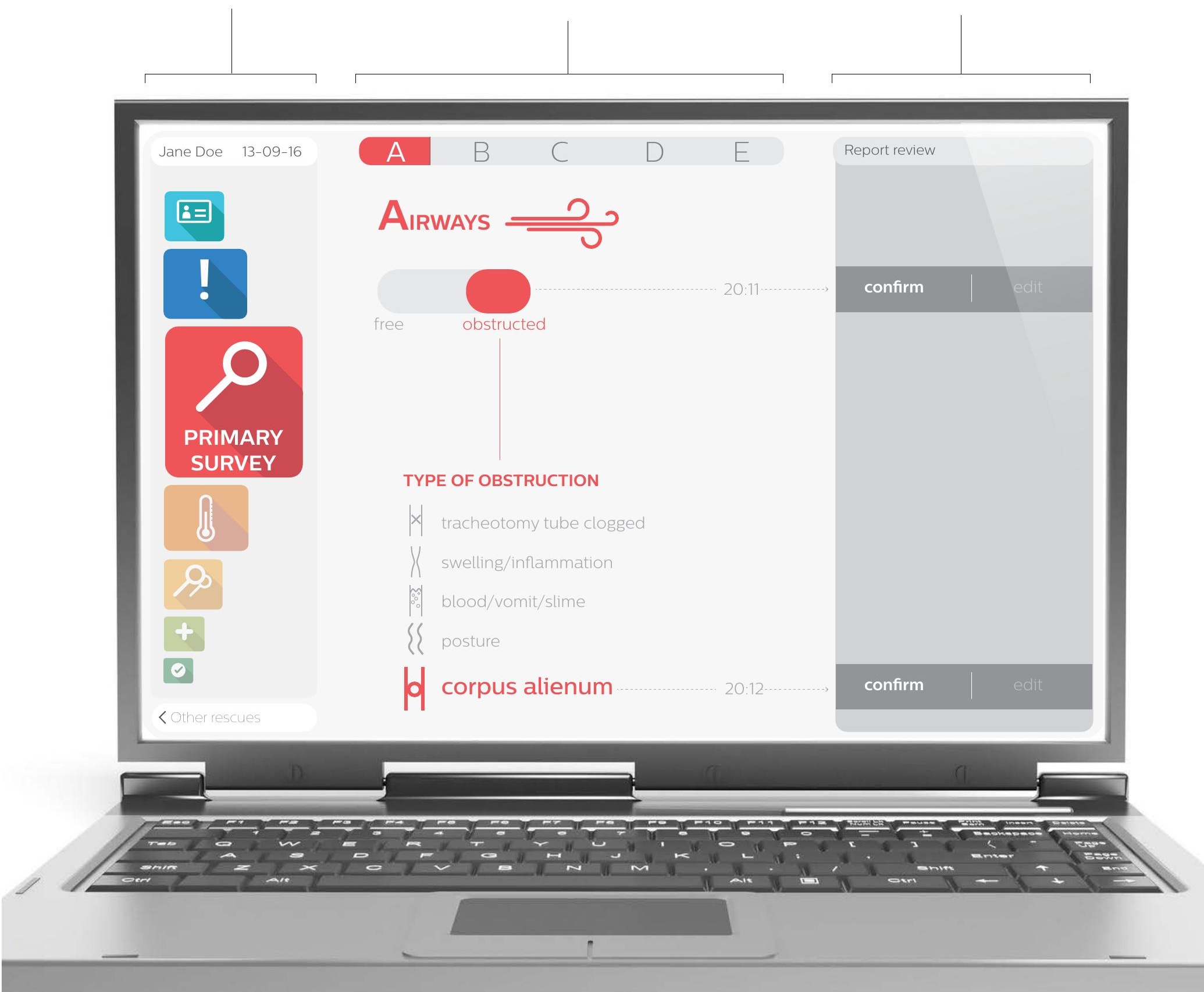




\section{Sound design}

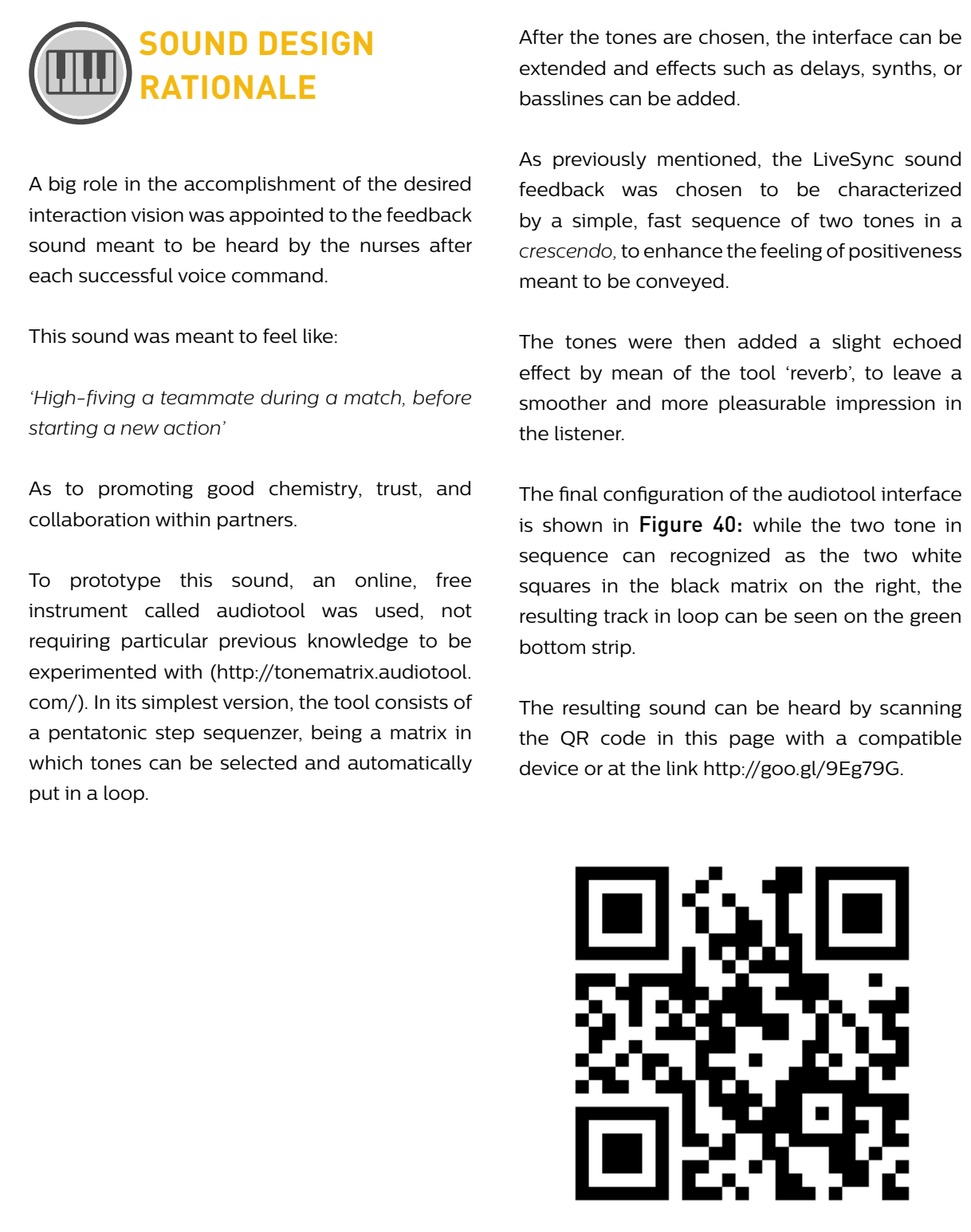

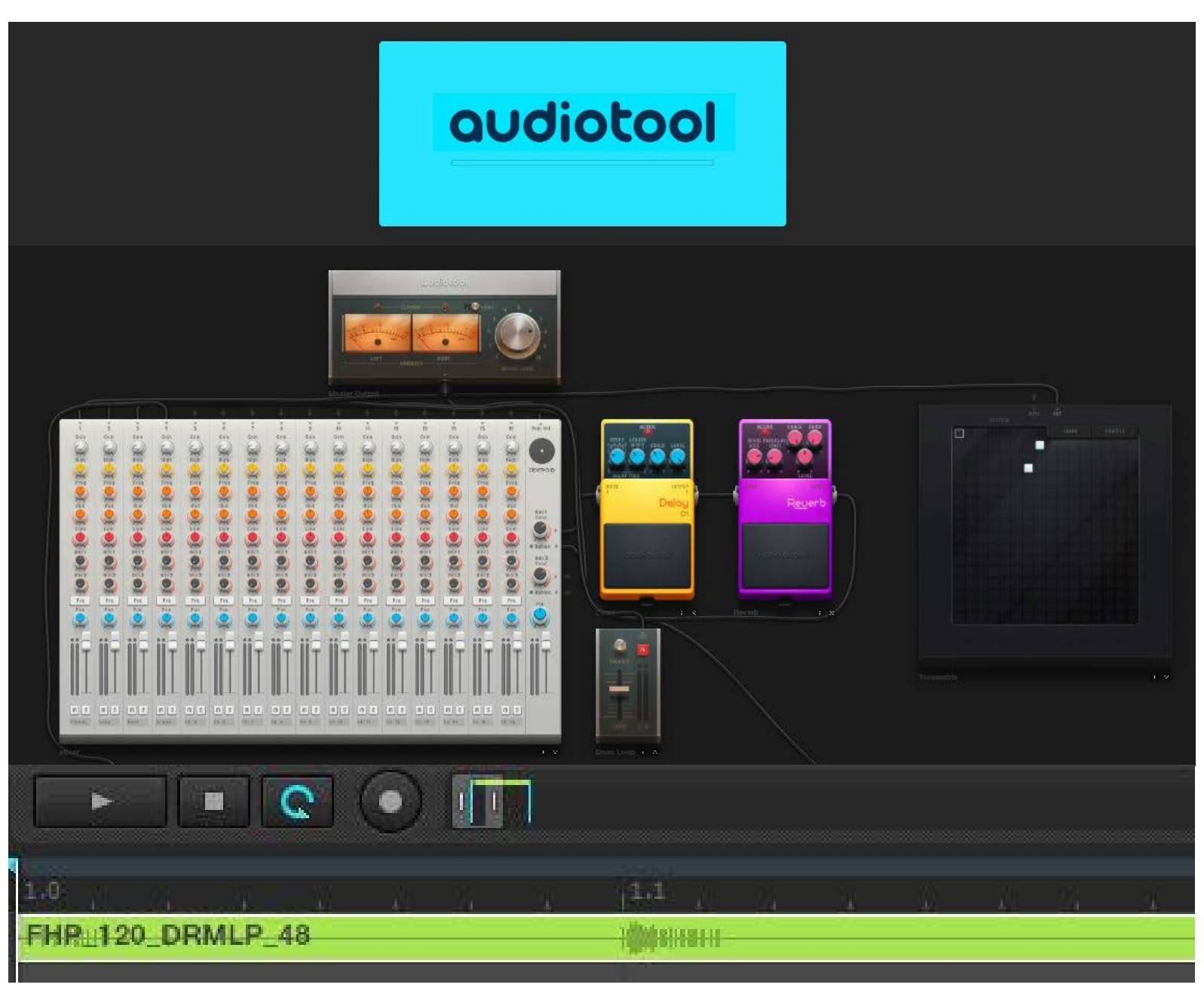

Figure 40 The making of the feedback sound
in Audiotool. 


\section{Cycles of use}

Introducing the Livesync in the work flow

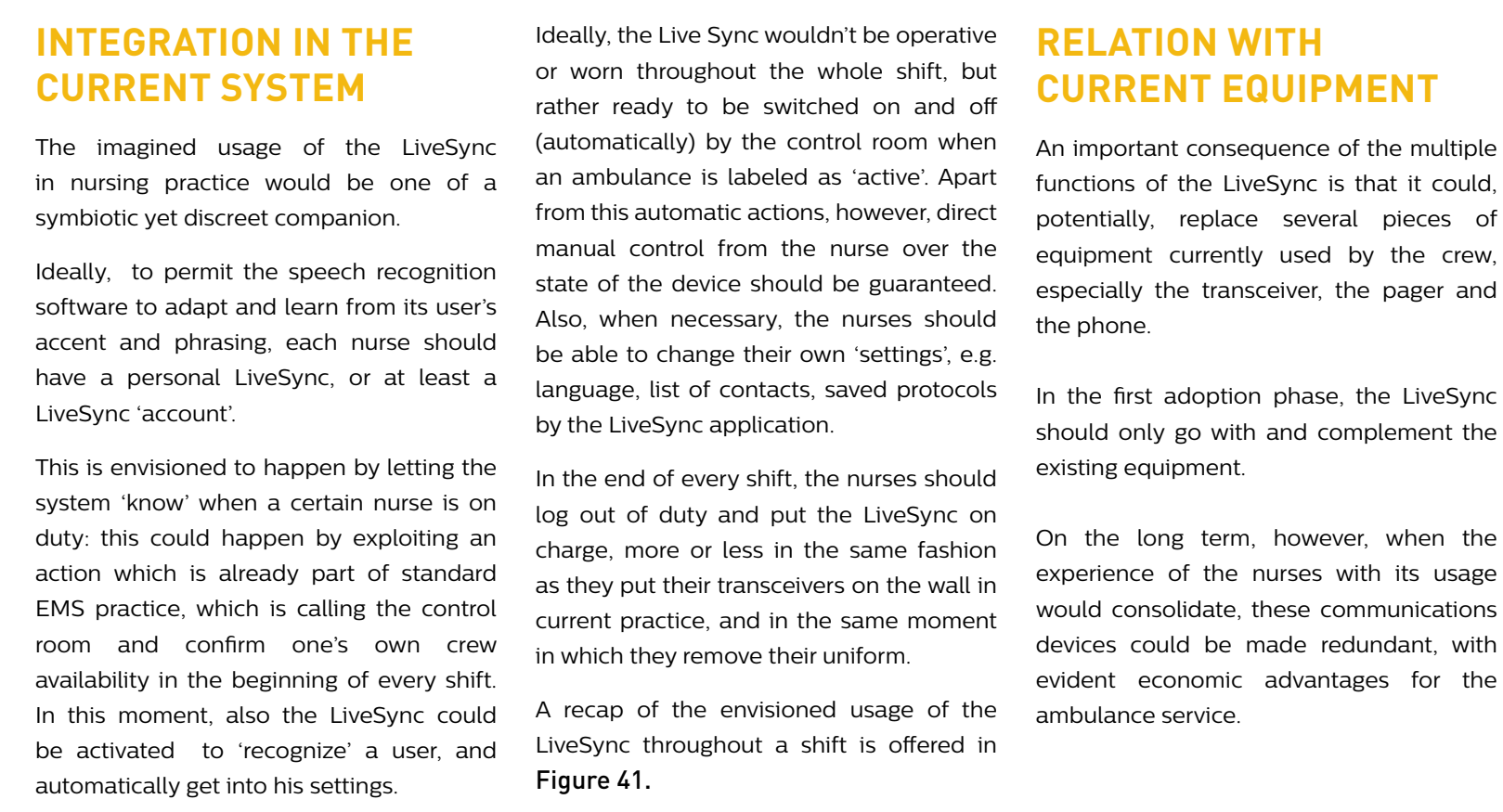

automatically get into his setich

SHIFT TIMELINE

Figure 41.

\section{Embodiment focus}

So what did you design?
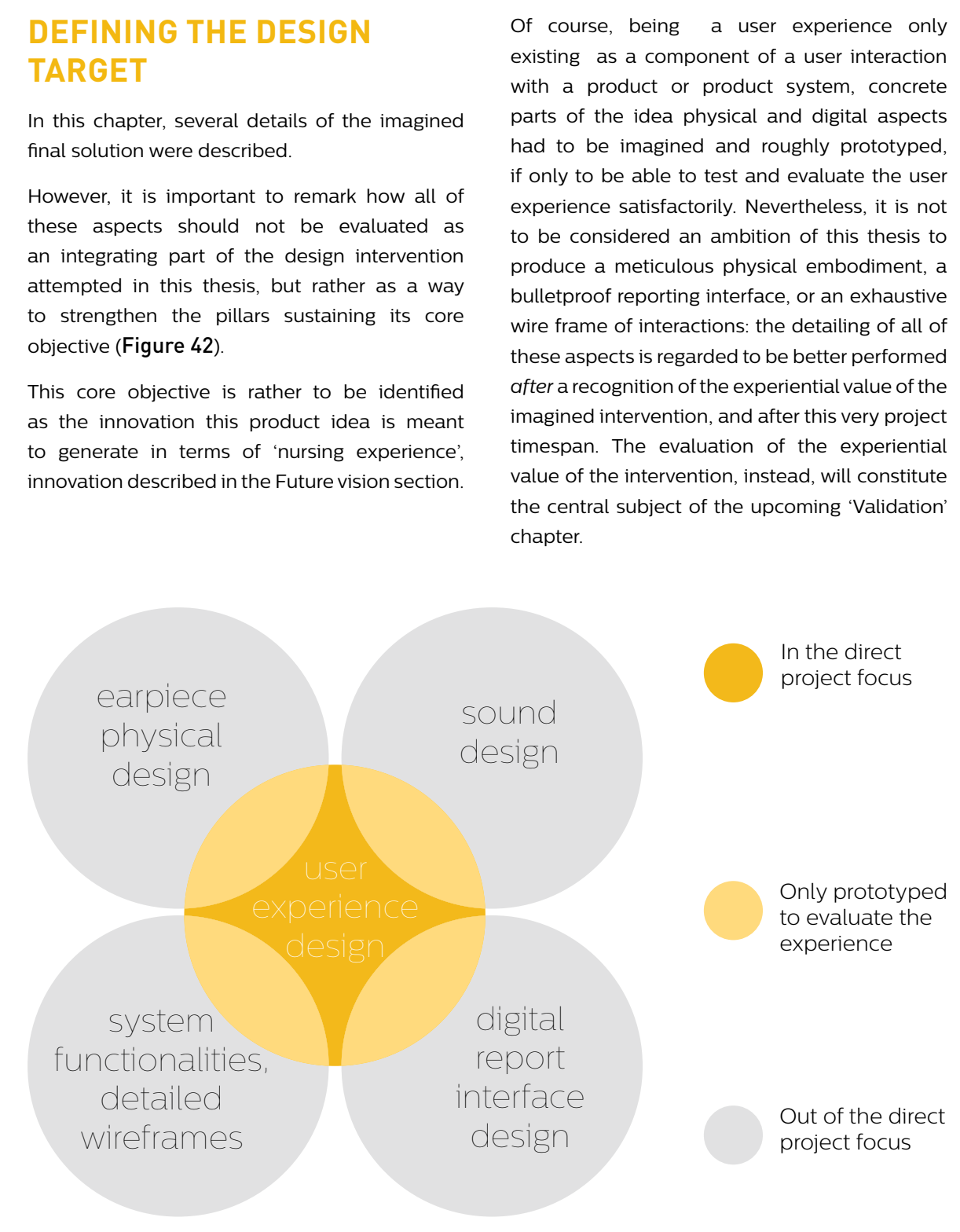


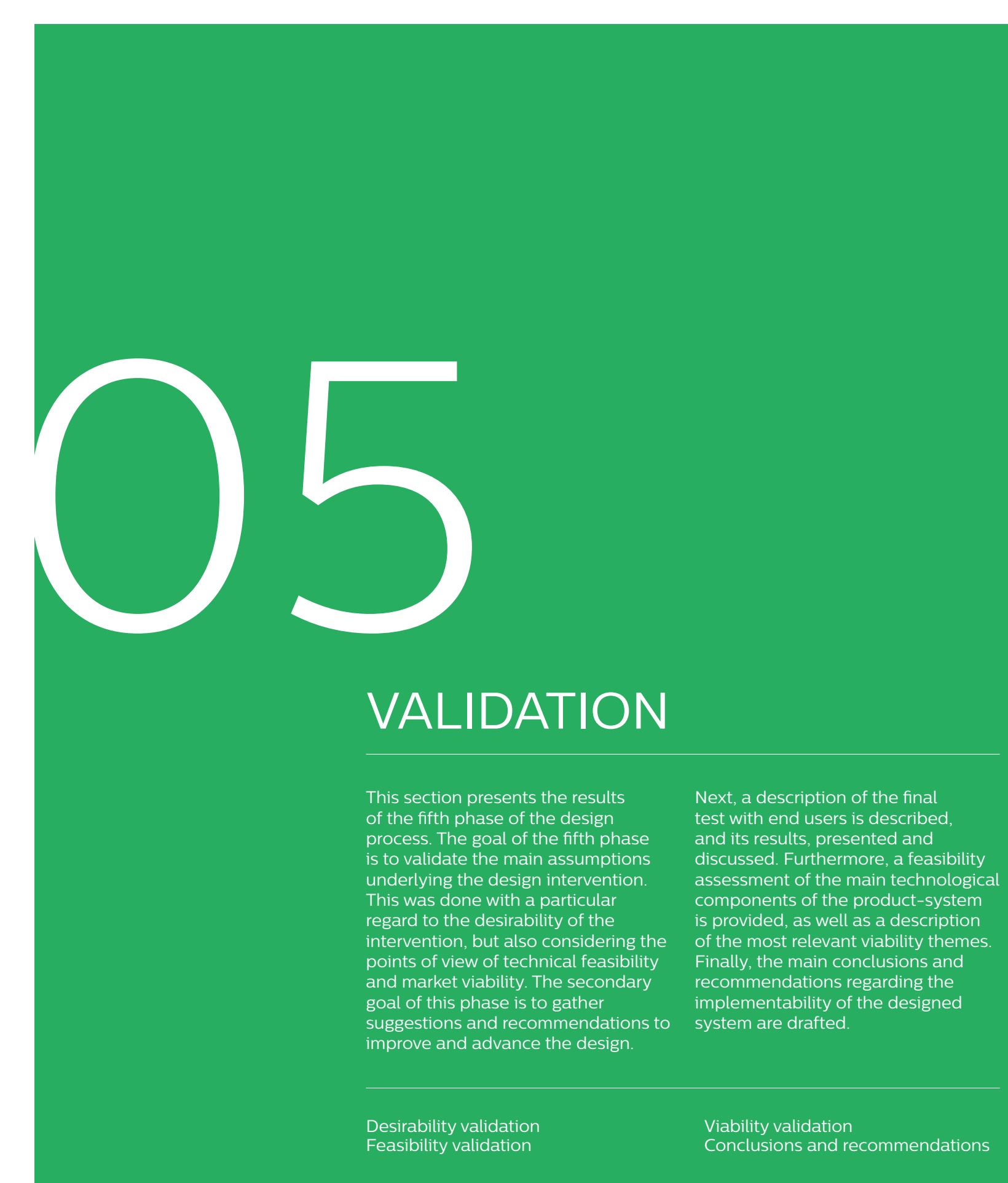




\section{Desirability validation}

\author{
Does this work for ambulance nurses?
}

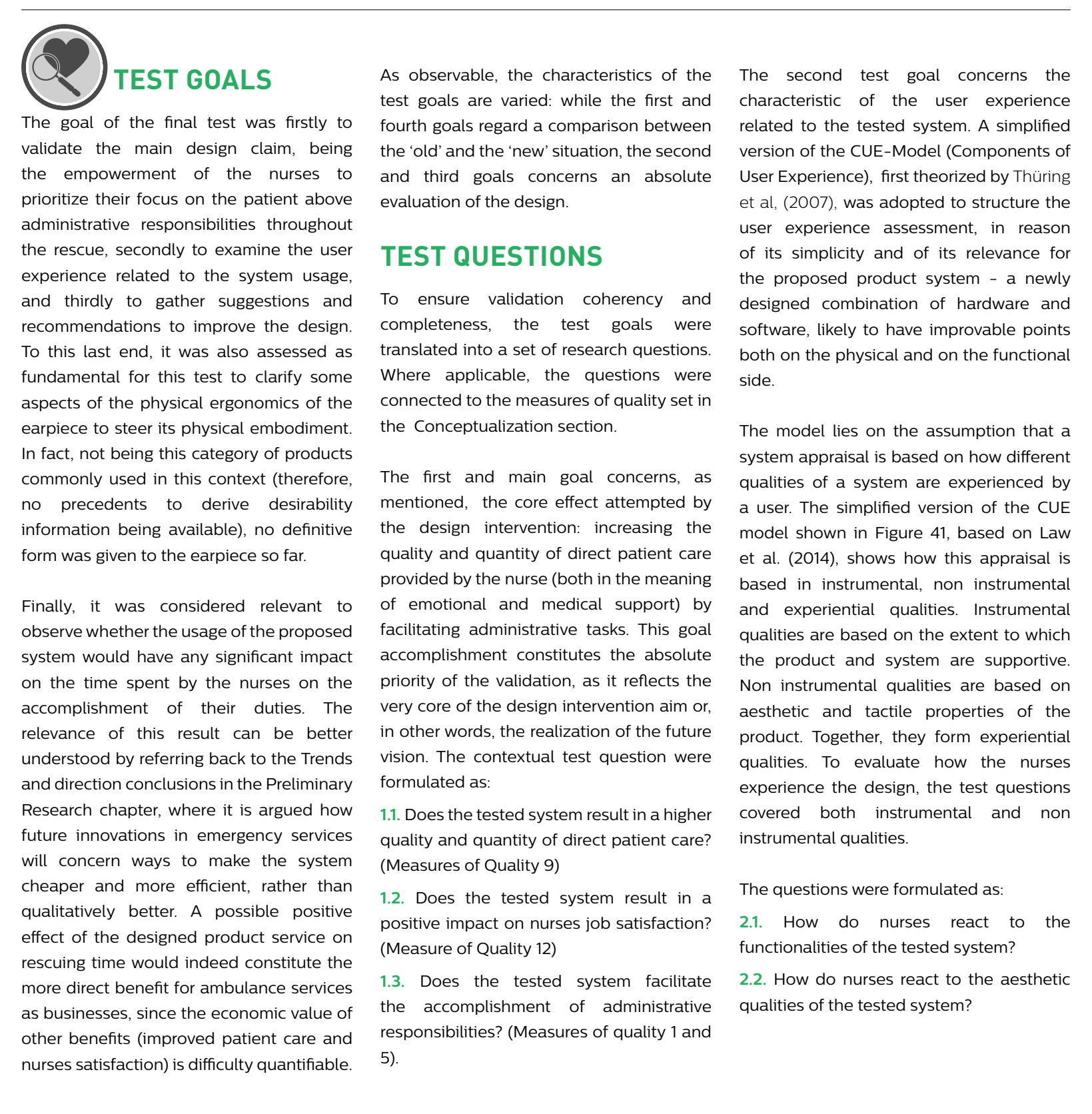

In addition to this, it was assessed as fundamental to evaluate the impact of the possible concept desirability threats already identified in the features selection phase. Each question, including:

Is weang an earpece perceved as 2.3. Is wearin

perceived as distracting

2.5. Is conmanding through voice input

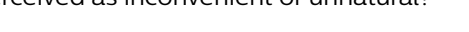

Furthermore, an open space to receive feedback of the derice, as well as sums related to the use the current design, was created by including the questions.

3.1. What are the main problems related to the use of the tested system?

3.2. Are there any suggestion about ways to

3. Which of the given earpiece forms would nurses prefer to wear on rescues? (see options Appendix XII)

4. Is the adoption of the device into the current

practice perceived as difficult?

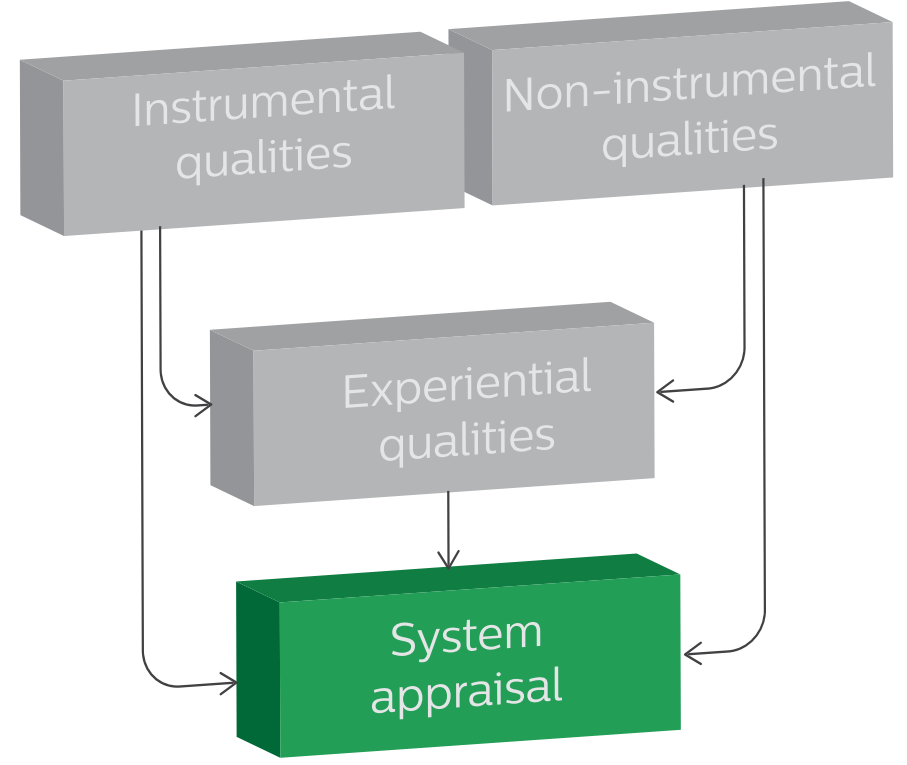

Figure 43 Model for system appraisal
(adapted from Law et al., 2014)
Finally, the impact of the system on the time of accomplshmment of nurses' tasks was observed

4.1. Does the usage of the designed system result in any difference in the accomplishment time of

PARTICIPANTS AND

CONTEXT

The test involved six ambulance nurses as participants. Only one of them was previously pholved in the user research, but did not know in order nof to bias the test. this was preferred of participants previous opinions of the concept

The testing was conducted as a sequence of simulations of a same emergency case. to this end, it is very relevant to note the high familiarity of ambulance nurses, compared to other users categones, to behave realistically in simulated stuations. In fact, their routine professional 
The nurses were individually asked to

Type of emergency The task of activating the sound feedback The kind of medical mergency to simulate was assigned to a Dutch-speaking test was decided to be a traumatic iniury and observer, instructed about the possibe specifically an ankle displacement. This keywords to react upon

anbulance center(now den wag), whereform leam were present. one acting as a patient, vo as modrators, and one managng the Theo recording and other testing supples. each participant upfront together with the functionalities and expected interaction of the tested device.

\section{MATERIALS AND}

EQUIPMENT

The material needed for the test consisted of an existing wireless earpiece, a smart phone connected to the earpiece, a application, and test documentation devices such as cameras and tripods Additionally copies of the after-test questionnaire and of the detailed protocol were kept available for continuous reference.

\section{PROCEDURE}

The test was built as a video-recorded simulation of an emergency case, in which
participants were asked to try and apoly the main functionalities permitted by the LiveSync.

To ensure comparability across the results of the different tests, it was attempted to equalize all of the factors not directly much as possible. In particular, the team member acting as a patient was instructed to adhere to the same symptoms during every test.

This decided, several specific details of the set-up had to be pondered. An overview
of the main variables choices and their of the main variables
motivations is offered nex cision was based on the several points:

Pre-filled report check simulation

In the system functioning as designed, the information collected by the earpiece would be automatically sent nto the digital report difficult to prototype effectively within the time and resources limits of this project. was decided to render this effect through 'Wizard-of-Oz' techniques. This was made by using a pre-filled report and providing it to the nurse after the rescue, as if it was automatically completed. The nurses were then asked to check and confirm the prefilled informations, as it would happen

The detailed protocol of the test is available at Appendix XII. In Figure 42, a summarizing visual of the complete test set-up is provided. exact case, constituting a great he for the researcher in forecasting the test's most probable of measurements taken, kind of questions asked to the patient, etc.)

\section{Test Language}

The interview following the rescue simulation was conducted coherently wit The operative parts of the test were test questions was covered by at least on decided to be conducted in the nurses after-test survey question. A complete

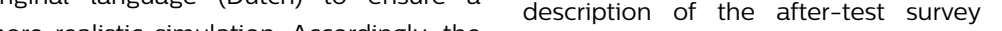
avallable in Appendix XII

Sperer conversely the after-simulation ANALYSIS PLAN

questionnaire was designed and conducted English, to guarantee the possibility from he test moderator (a non-Dutch speaker)

\section{oice input simulation}

Questionnaire results and video recordings of all of the tests were reviewed, whil takng note of every plece of fromatio

These results were in turn worked out to To simulate the system discovered potentialities the earpiece, it was decided to resort to an pagaph nurse, and connected to a sort phone reproducing the designed 'crescendo' sound feedback whenever recognized appropriate
FOLLOW-UP INTERVIEW test

phases
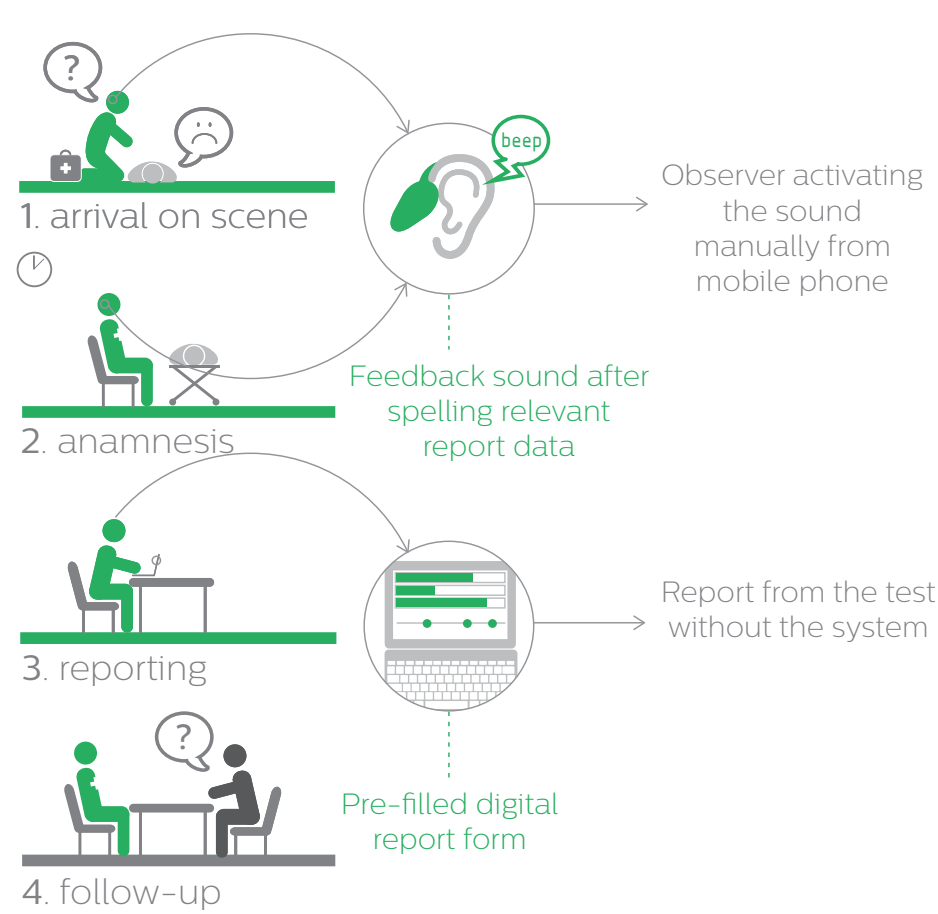

interview

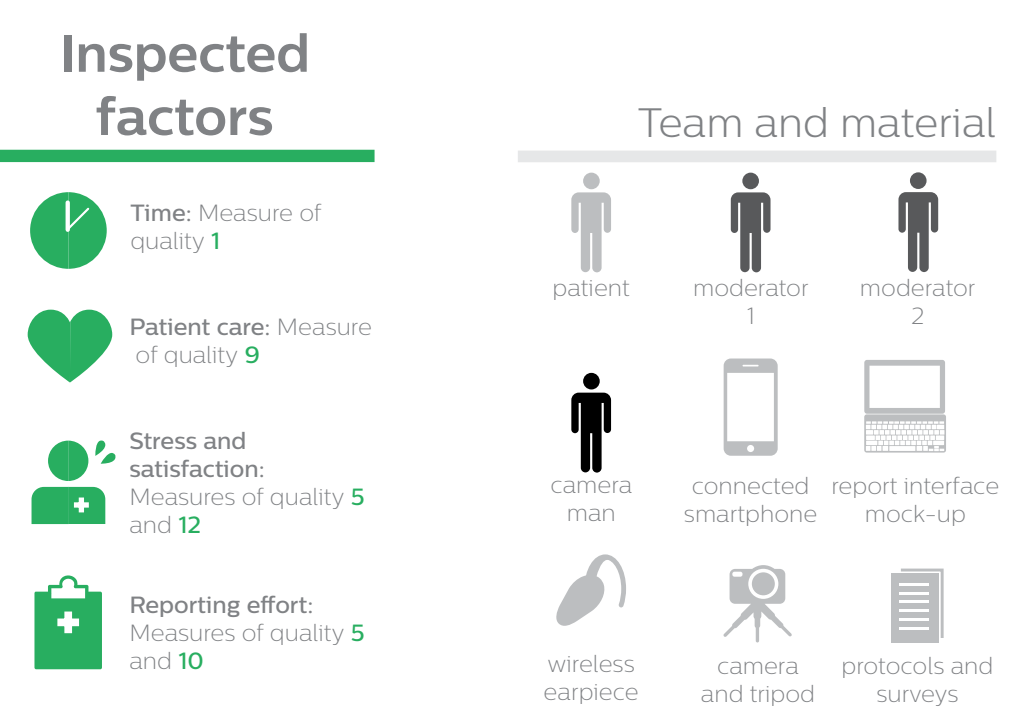

Figure 44 Test set-up summary 
TEST RESULTS

The test execution didn't present particular difficulties, as the participants understood
the intended functionalities of the product system without major resistance and could simulate the intended product unctionalities rather naturally.

The preliminarily observed tesyt results participants welcomed the expected advantages brought by product, and could drawbacks of it in a real scenario

Additionally, each research question result is discussed separately.

11. Does the tested system result in a higher rate of direct patient care? (Measures of Quality 9)

The opinions of the involved participants on this point were articulated around the variable of attention reserved to the patient.
Most nurses saw a strong point for patient care in the fact that the device allows them to focus with their full capacity on the
live case as it unfolds. Two other nurses, live case as it unfolds. Two other nurses,
conversely, argued that regardless of how easier it would be to acomplish the eport the own capability of the nurses in their job would not be affected, and bad' nurses would still deliver insufficient patient care. This argument, although very relevant, is not surprising: as the approach of the product user experience was never to replace or interfere with the personal capabitres and expertse or the nurses, but

During the test, other unexpected ways in which the use of the device could impact on patient care were brought up. One of continued use of the product system cout ealistically have a long term effect of augmented compliance to protocols and checkilsts, due to an increased awareness spoken out instead of kept in mind.
To give a concrete example, the nurse This kind of considerations brought two case, in which the first of the interviewed nurses to evaluate the expected assessment is the ABC (airway, impact of the product on this factor as

the patients vital signs. In the interviewed 1.3. Does the tested system facilitate

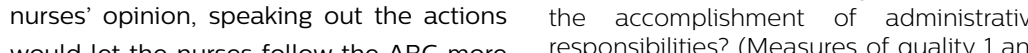

This last observations marks a very deep could determine on the long term of the the nurses who didn't find this difference . the system as envisioned, different report remarked that, in his opinion, the report strctres would eventually generate itself would however be presumably better different nurses approaches to cases. This while maintaining the same effort, and specifically more complete and accurate,

$\begin{array}{ll} & 2.1 . \text { How do nurses react to the LiveSync } \\ \text { regarding thation in a scenario of real productionalities of the tested }\end{array}$ implementation

1.2. Does the tested system result in a The interviewed nurses, although having job satisfaction? been illustrated the concept in all details, (Measure of Quality 12) The main arguments nurses found to one of the functionaltities of the designed ent affect their overall iob satisfaction can be focus on this aspect dring the following mmarized on these lines: interviews

I would do less reporting, therefore do my job

things I don't like in However, a definite interest for the and faster, so comilities enabled by the device on the

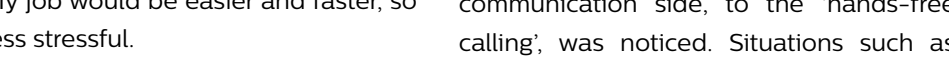
I would get the chance to spend more mass casualties, in which many ambulance quality time with my patents, which have to cooperate in a joint effort, were whe tangible difference.

enthusiasm and fewer comments were a reassuring nurses observations, give Less the expectable reported on the "instant protocol showing impact of the designed product-system functionality, although the reasonin on ambulance nursing as a profession, it behind the function was never objected. is worthy to briefly look back at the stress' $\quad$ 2.2. How do nurses react to the aesthetic sources identined in ambulance nurses as qualities of the tested system? Characters in the User Research section It is possible to summarize the participants not impacted unon by the device Non- the physical product as not to make it tor cooperative patients, traumatic events, evident nor to mate it look live a hearin physical distress, mental burnout, wou still make a very challenging profession out of ambulance nursing.

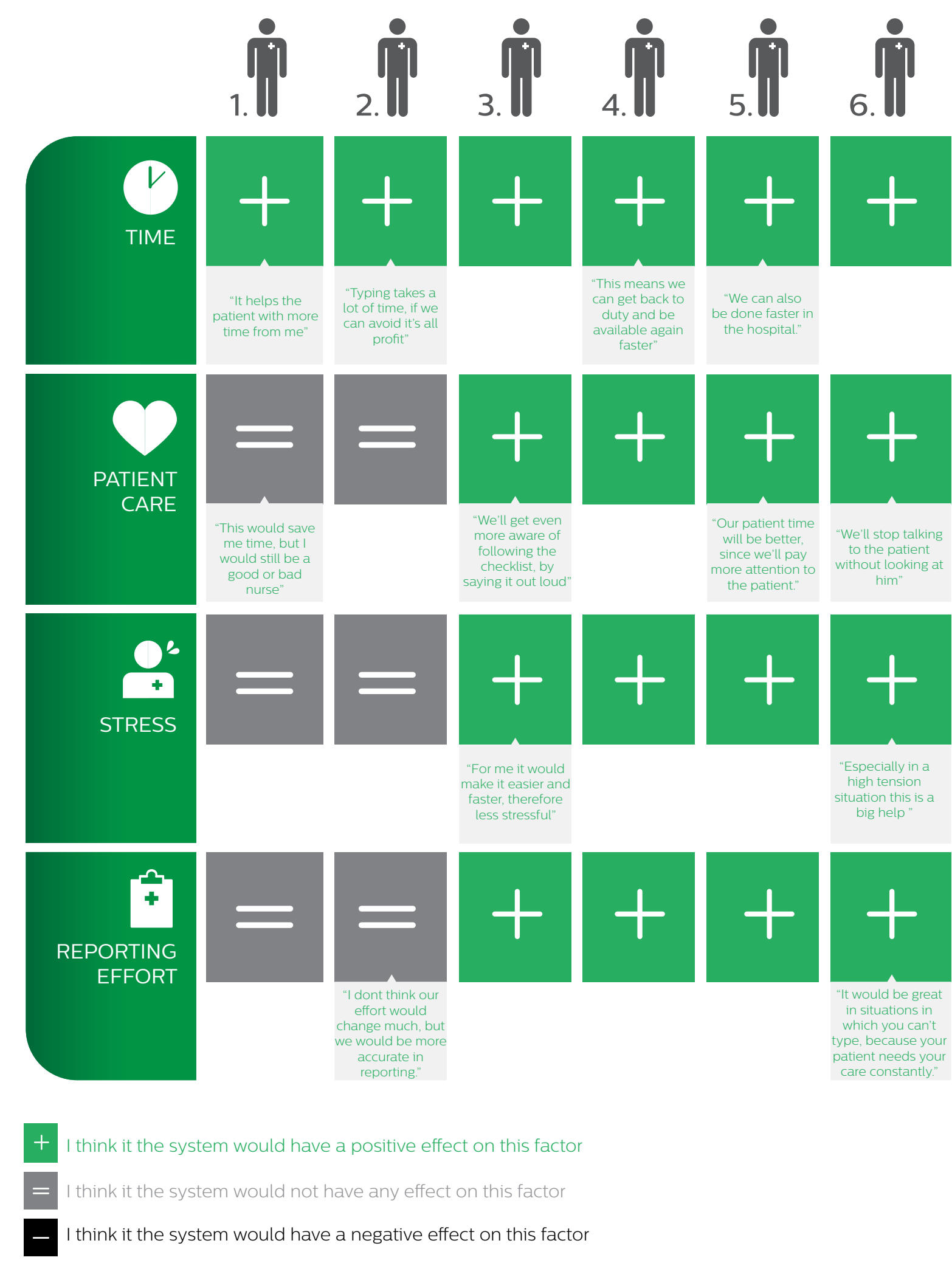

Figure 45 Test results summary 
2.3. Is wearing an earpiece perceived as
uncomfortable?

The idea of wearing an earpiece when on duty didn't seem to bother the participants in itself, although many recommendations were expressed on the fact that the physical most of all, stable

2.4. Is receiving audio $\mathrm{p}$
perceived as distracting?

The interviewed nuses could guse, on this point, quite informed opinions, as this simulated in the test Surprisingly for the researcher the participants seemed to familiarize with the feedback sounds mechanism, and denied an effect of distraction of this input on their mental processes, right from the first testing experience itself. An interesting metaphor of the experience resulting from the test was given by one the participants, who from the earpiece as one of a clock ticking: subtle familiar and totally non-influent on the listener's focus unless desired

2.5. Is commanding through voice input
perceived as inconvenient or unnatural? perceived as inconvenient or unnatural?

The possible social discomfort, resulting from the action of 'speaking into a device' lopic in several interviews. Many nurses minimized this possibility, mainly in
virtue of the fact that repeating the most virtue of the fact that repeating the most elevant information to their partner is already a part of ther everyday practice which the atron would insteal appears evidently artifcial (es not in presence of the partner, or when articulating detailed sets of keywords such as: patient vehicle: (car'). However, when needed, none of the nurses saw a problem in explaining the
motivations of their behavior to the patient. 3.1. What are the main problems you identily
as related to the use of the tested system? Several possible usability drawbacks of the syed me main ones were expressed as:
The possible limits of the voice reporting function in complex emergency situations, in which many patients or many ambulances nurses would be present on a same case. results and the possible difficulty of manaing possibu cally measurement first spoken in the system and then corrected).

The issue of the volume of the soun feedback, being possibly insufficien in loud environments (e.g. concerts)

.2. Do you have any suggestion abou
ways to improve the current design of the

Making it easier to wear

. to avoid the risk of losing it.

Leave the ear free, to permit the use of the stethoscope (e.g. by using bon conduction earpieces) mplement a self-adjustment of the

3.3. Which of the given earpiece
would you prefer to wear on rescues?

All of the participants chose the third option

This choice was mostly motivated by the 列 discrete and comfortable appearance. 4. Would the adoption of the device

warmly recommended To this end, a very pertinent observation a gradual adoption of the system, and made by a test participant is that the an adeatrate rang of and support in the the saving effect would vanate largely the seses, depending on in the systom insteme a major problem their complextly and their data density. the fist). the effect of the device on reducing not contrast with any current practice and the reporting time would be very evident its adoption can be as gradual as the nurse when compared with simple cases such wants (e.g only entering the medications at as the simulated ankle displacement. A first, then the measurements, then including recommendation for these possible furthe ther functions such as the hands-free quantitative tests would be, therefore, to calling or the monitor control)

quantitative tests would be, therefore, to a comparative evaluation of the effect of oad spectrum of (n)

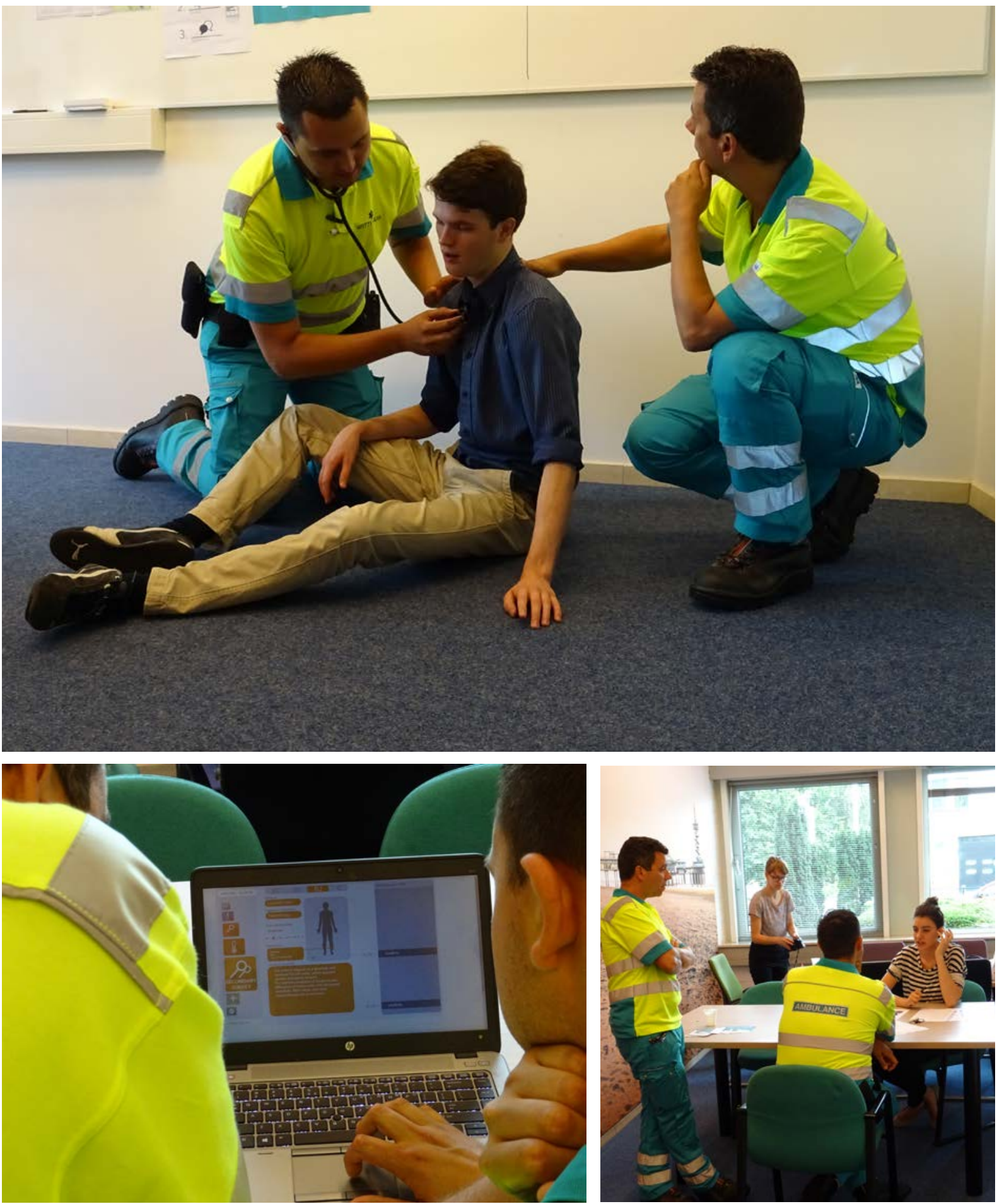

Figure 46 Test phases 


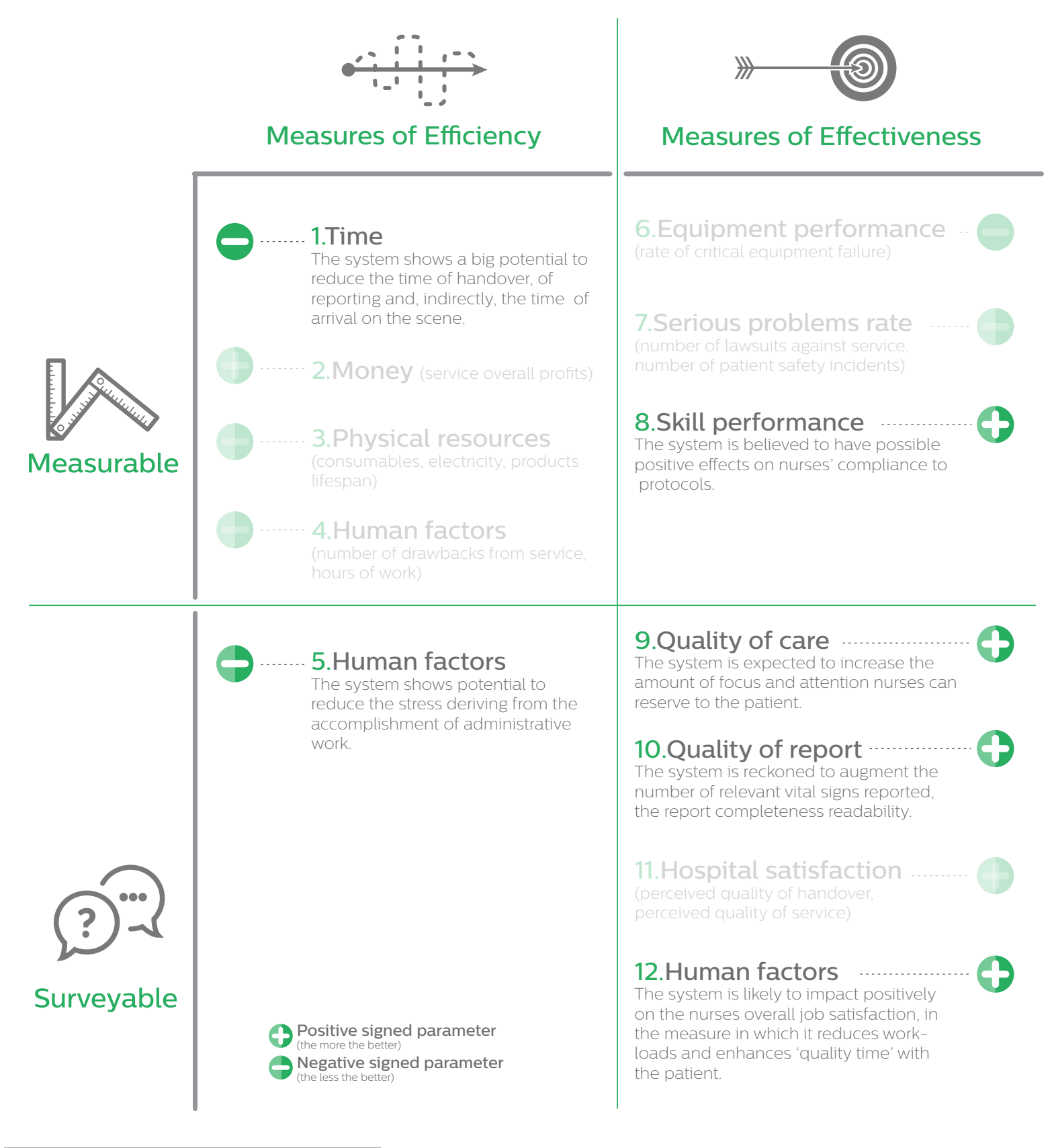

Figure 47 Measures of quality on
which the system is estimated to
impact positively
TEST CONCLUSIONS

The test suggested the designed systen to be overall desirable for the nurses and presenting a potential to improve the quality of ambulance services on several
levels. The measures of quality on which levels. The measures of quality on which
the adoption of the system would impact the adoption of the system would impact
upon, and the nature of this impact, is summarized in Figure 47 .

Out of these recognized points of value, a few (shown in Figure 48) were welcomed by the participants with particular warmth. Nevertheless, several some problem areas these advantages, as listed in Figure 49.

Further cycles of design iterations and test, Further cycles of design terations and test, areas are recommended as a next step in the project. One immediate decision that can be taken after this first test, due to the clarity and reasonableness of the results, is the usage of bone conduction earpieces for

further embodiments, to leave nurses' ears
free to use the stethoscope and to listen to

free to use the stethoscop environmental sounds

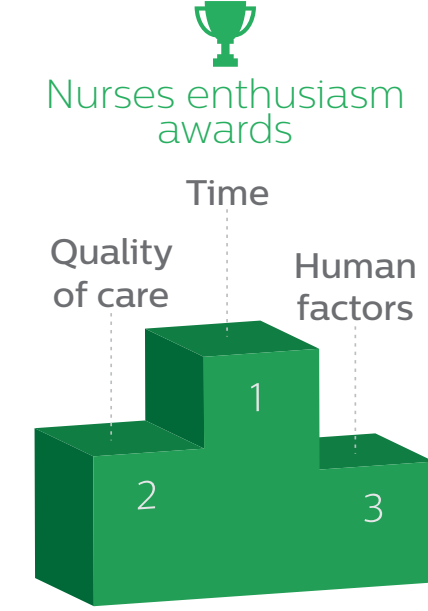

Figure 48 Potential areas on which the interviewed nurses
strongest appreciation
TEST LIMITATIONS

insights would probably of unexpected tes meaningful within the boundaries of the recognition function is integrating part of 列, tested technology.

semp the encouraging results

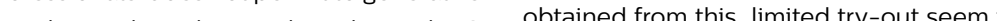
further, more thorough user evaluation. inions, feelings and preferences. Furthermore, the very setting of the test The main recommendations here offered for such a testing are summarized as: In not to be considered representational
of reality: what happened in a controlled environment, in a visibly acted-ou stuation and under the sight of several elike situation.

Fnally, it has to be remarked how the est was conducted using a heavily experience was only roughly resemblin he one imagined.

\section{Design a test set-up allowing for} current nursing practices.

Using full-functional prototypes of

the earpiece and of the reporting software

\section{Main problem areas}

3 Social discomfort of speaking to a device

Input conflicts (e.s. same report field being filled twice.

Physical embodiment resulting too cumbersome. too

t Aesthetic appearance resulting too evident or too

Figure 49 Main problem areas
emerged from the test. 


\section{Feasibility validation}

Is the described technology available?
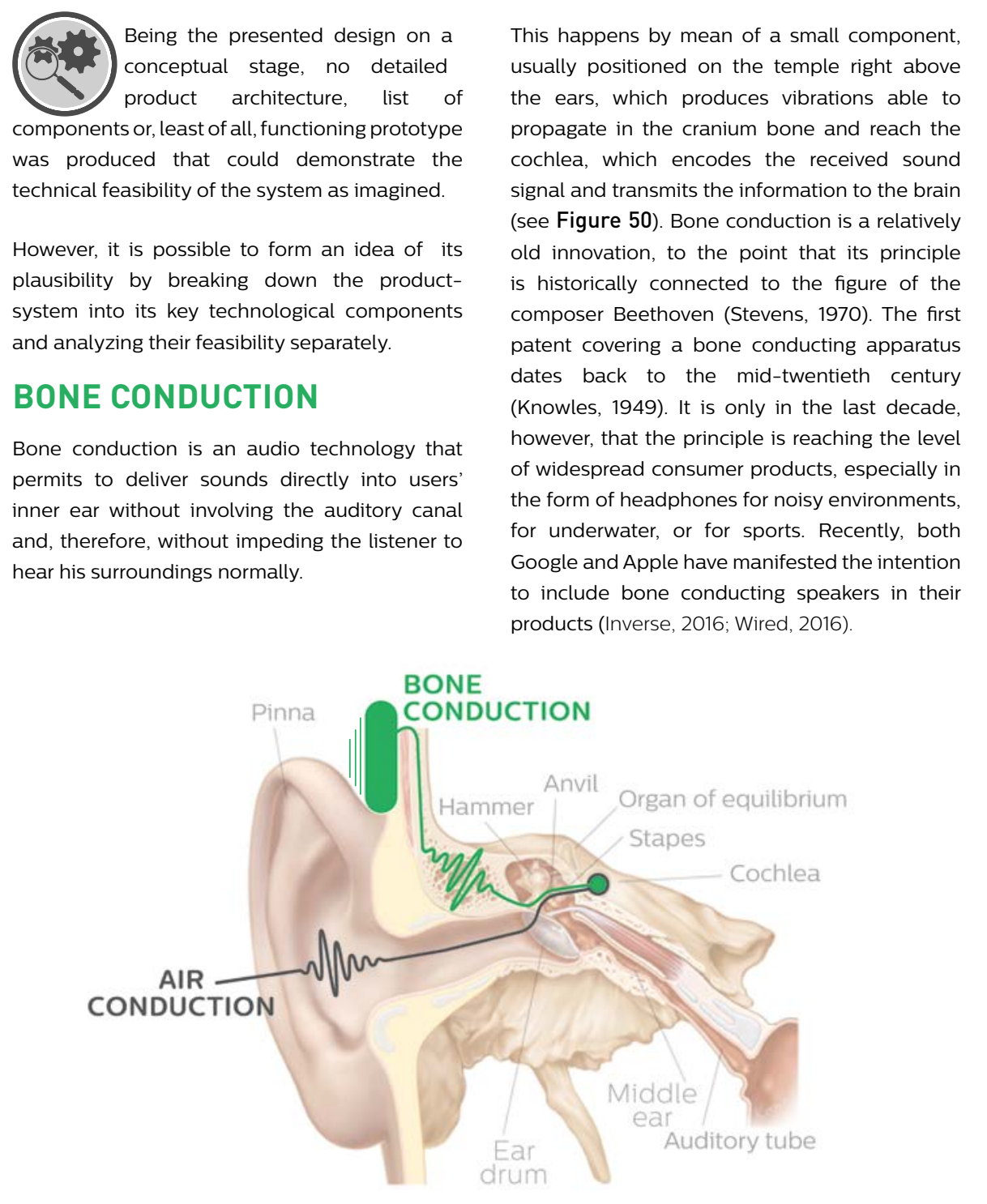

Figure 50 Bone conduction's
'alternate pathway' to reach the

alternate
cochlea.
SPEECH RECOGNITION

A voice recognition software, being system able to sort automatically words out of sounds, Consist essentaally or wwo parts.

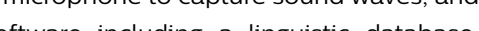
The software able to analyze the sounds is. arguably the core of the technology. Its principle is to break down the sound from the microphone into small speech factions called phonemes: the English language counts 44 of them. The order and succession 4 which he phonemes are found permits the sophisticaled speech admissible approximation the ext wh an being pronounced, For homophone words, such as 'cell' and 'sell' the software sorts the context and syntax of the phrase, to calculate the best probability to recognize the spoken term-just as we humans do.

Modern speech recognition softwares can

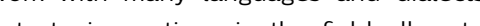
Latest innovations in the field allow to percent (Consunese

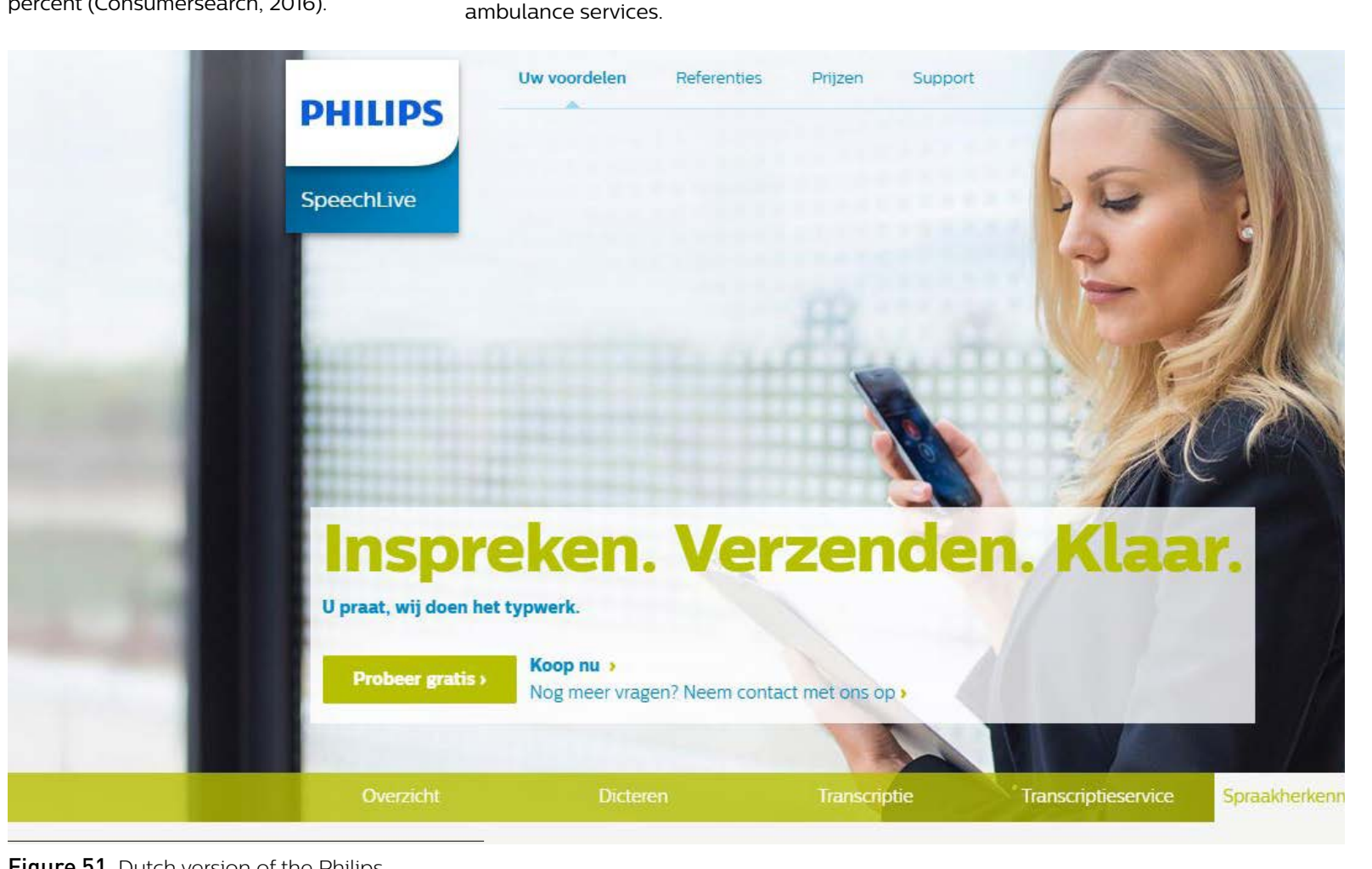

Figure 51 Dutch version of the Philips
Speechlive landing page
This is made possible by machine learning However, it seems realistic to expect, in thes, th has to be reminded how, while as anticipated in the Trends and Direction by by in primarily be able to Netherlands (Dutch Ambulance service, (ecognize reporting -relevant terms which 2016) and in the us (Hospitals 2016) uld be already 'prioritized' in its linguistc database. elongs to Philips' RECOMMENDATIONS

DIGITAL CONNECTIVITY

he system to perform as imagined, all However, a detailed engineering appraisal of the different technological components is recommended to evaluate crucial
previously mentioned (the earpiece factors such as the dimensioning of the microphone and speaker the speech fars recognition software and the report resistance to the extreme conditions faced application) should rely on a continuous in an emergency care environment. stream of reciprocal connection. This possibiry seems not to bewidelyavalable at the present state of advancement, in Altogether, the core principles permittin the imagined functioning of the Livesync seem not to be realistically unattainable

$$
-
$$




\section{Viability validation}

Can this be put in the market?

A reliable assessment of the market viability

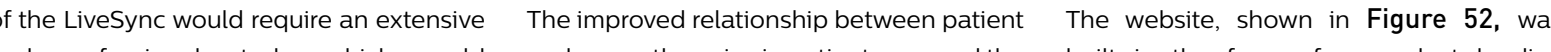

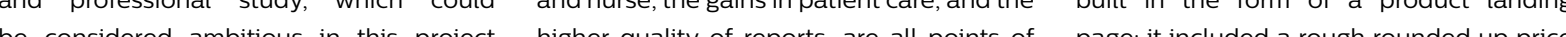
cospan and ultimatey out of its scope. value somehow proven to represent strong indication sugested by Philips experts $(50)$ Consequently no detailed business plan or advantages for the users but whose overall cents per ride) and a small guestionnaire definite conclusions will be here expressed financial weight can only be calculated on addressing the propensity of visitors to for the convenience of Philips to take the long term. purchase the product -or to suggest its actions towards implementation. However, to add a more concrete perspective to the overall value of the idea, a few business considerations are hereby provided which
would be relevant for a hypothetical market would be relevant for a hypothetical market These reflections can benefit from insights constitute a last exploitation of the close perspective on the EMS world that was built in the making of the project.

\section{(1011) PRICE}

combination of hardware product and software service as the one proposed in this thesis poses a plethora of difficulties The first and more evident dilemma derives
from the novelty of this kind of solution from the novelty of this kind of solution In the context of application. No direct
competitors being present in the current market, it is possible for the LiveSync to move across an extremely wide range of pricing discretion, a typical phenomenon resulting from maximal produc differentiation.

To cope with such a challenge, a linear The 46 responses from the website, after The rather favorable. All of the ared to be (d) a this steps to be taken for he prang or do Estimation of demand

2. Decision on market targets.

A small-scaled example of a possible ivesync is offered next, followed by som considerations on the preferable mark

STIMATION OF DEMAND To have a first exploratory estimation of the market interest towards the kind of designed solution, a website was developed (see the UX vision and the specific functions of of advantages offered by the LiveSync for the purchaser, when we intend this as the try-out of the device in their amena a service. When inquired about the main ay saw in the idea the esponders showed a sensible difference of focus depending on their professional role. Generally, ambulance nurses saw the greatest advantage in the time saving effect of the device (especially regarding activities While and down long medcalons lists a good solution to improve reports quality this might suggest that a multifaceted value points of view of many stakeholders, should e proposed solution, also by mean of a be integrated in the product business plan demonstrative video
LiveSync

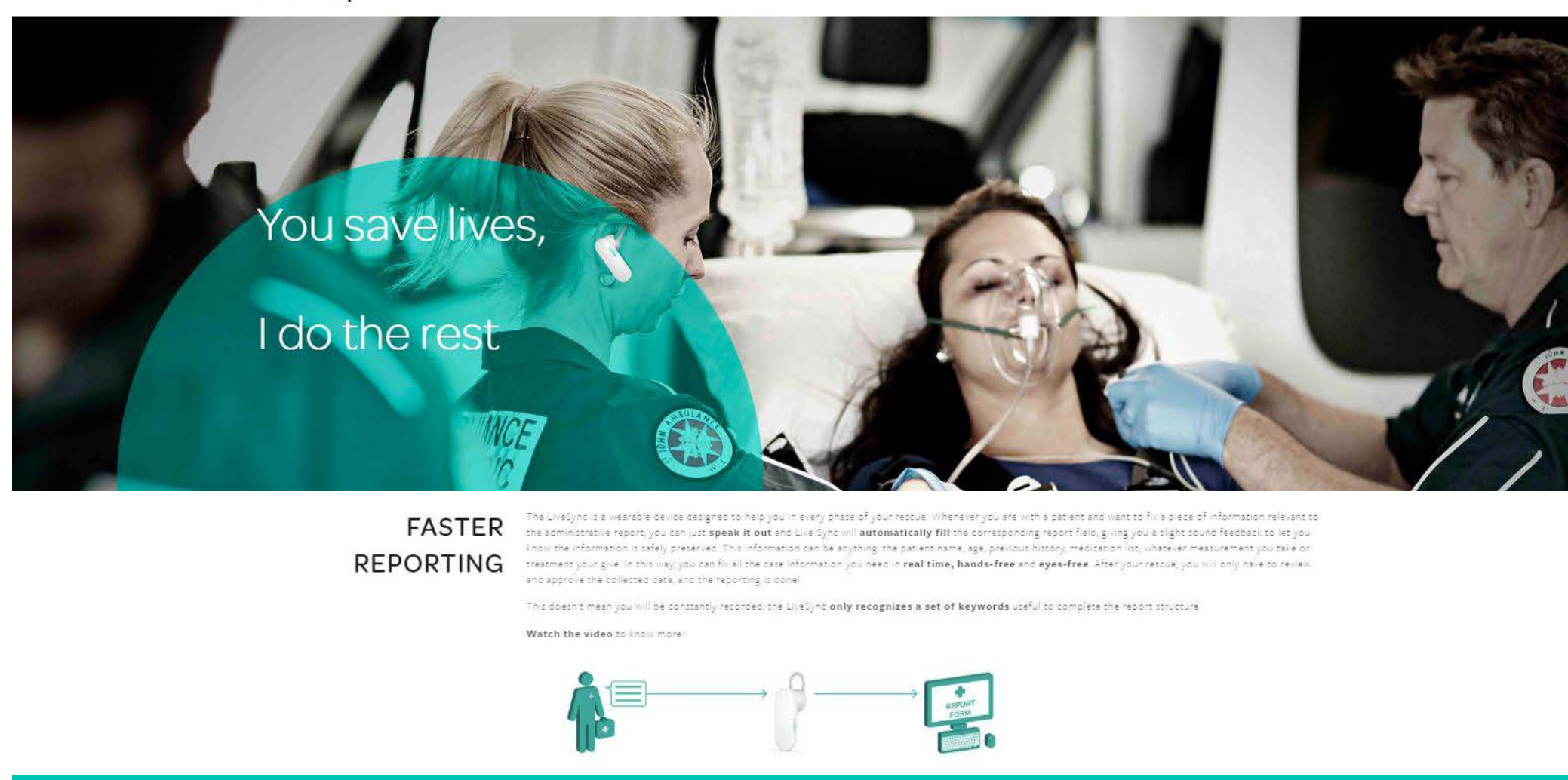

Livesync

(

EASIER COMMUNICATION

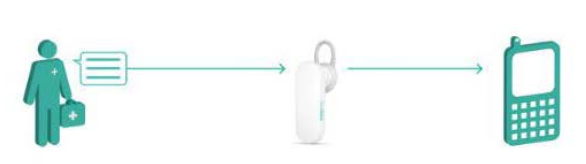

.all for 50 cents per case*

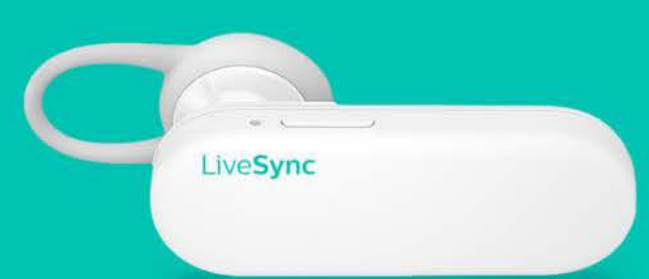


MARKET TARGETS

The decision of the market sectors to
address with the designed solution is an address with the designed solution is an
operation which should be prompted by considering Philips future ambitions, as well as its current positioning in the market.

From a theoretical point of view, Philips as a brand might have several good reasons to strengthen its position in the EMS sector: of the 'health continuum' (Philips, 2016) can make us imagine the potential importance
for the company to expand into such an important transition point in the healthcare system such as EMS. To give one example the possible strategic advantages especially in terms of data management and telehealth, we could consider how positively this could impact on the support given to chronic patients already involved
in the Philips hospital to home business (Philips, 2014). In fact, chronic patients belong to a category which is frequently
involved in ambulance rides, and that is now monitored and reached by Philips

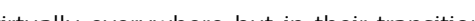
moments.

From a perspective of existing market coverage, however, it is noteworthy to remind that the current involvement of
Philips in the EMS market is mostly limited Philips in the EMS market is mostly limited
to the provision of stand-alone products such the provision of stand-alone products
such the Heart defibrillator or the Suresigns vital signs monitor. Moreover, would not seem to reach a market quota large enough to render the idea of an ggressive new product launch in the sector convincins

possible strategy to bypass this obstacle would be to get involved with other, intermediary parties already providing services. deally, these parties would be the existing CONCLUSIONS AND RECOMMENDATIONS

reports) to ambulance services. To them, To sum up, it is advised, to evaluate the
Philips could offer the Livesync (made actual viability of the proposed product chilps could offer the LiveSync (made actual viability of the proposed produc services

these companies in the US ld be Zoll (https://www.zolldata.com or Physio control (http://www.physiocontrol.com/), both firms with extensive experience and diffusion in the ePCR field would be, in this way, dramatically easier for Philips, as an agreement with a few big ePCR companies would result in a large number of users -as opposed to the realistically slow product placement imaginable in case Philips was to sell the Livesync, one to one, to the myriad of small

This strategy, linked to the concept of open business model, (Osterwalder, 2010) would under certain aspers, con as it benefit for all of the involved parties: A ther business aspects are finally given. o its data management business, the PCR companies acquiring an advantage fom their competitors they would not have he know-how to reach alone, and the oxprting experience without the need to hange their IT infrastructure.

This 'triple win' configuration would appea even more evident in case a reven sharing approach was chosen. This would mean that, out of the additional cost the PCR companies would charge ambulance sericesfor he Livesync provision, a certan percentage would be retained by Philips. A summanzing scheme of such a system actual viability of
service-system, to:

Conduct an extensive research cerce for a dance sevices a a Carefully evaluate the possible existing retailers of $\mathrm{EPCR}$ solutions for

After this, a cost estimation, taking into account hardware production and software

The comparison between the so estimated selling prices, the market dimensions and the development costs, togethe with broader considerations in terms of with a well-informed ide should provide profitability of the idea presented in this Ideally, the product should be covered protecting it from 'me-too' products coming to compete on the long term The production of the earpiec hardware could be outsourced, and the software part could be executed in-house

In case of actions in the Netherlands UMCU hospitat in Urechl, and the as they played a major role in the esting of the idea and they declared their strong interest in participating in possible product ativities.

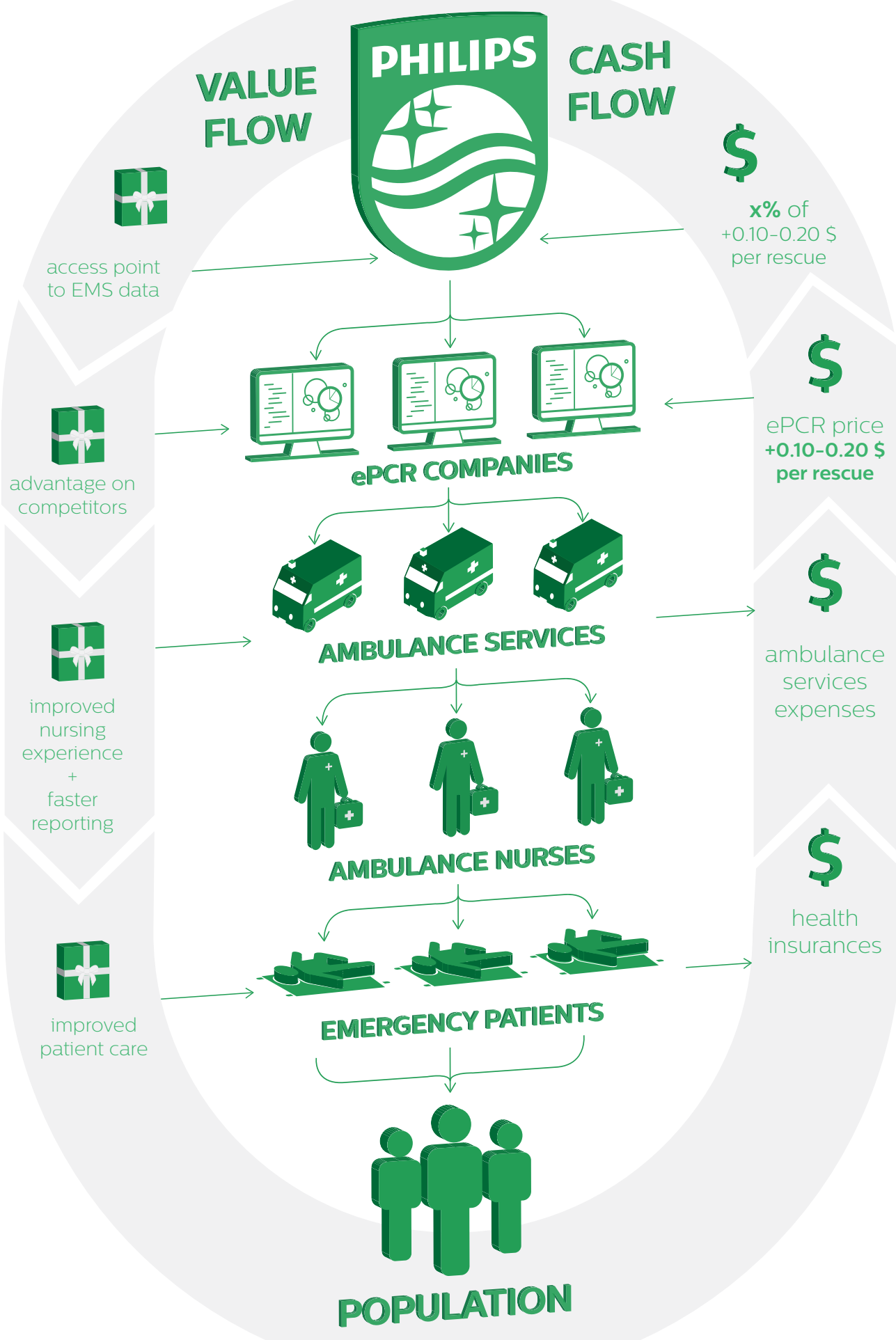




\section{Conclusions and}

\section{recommendations}

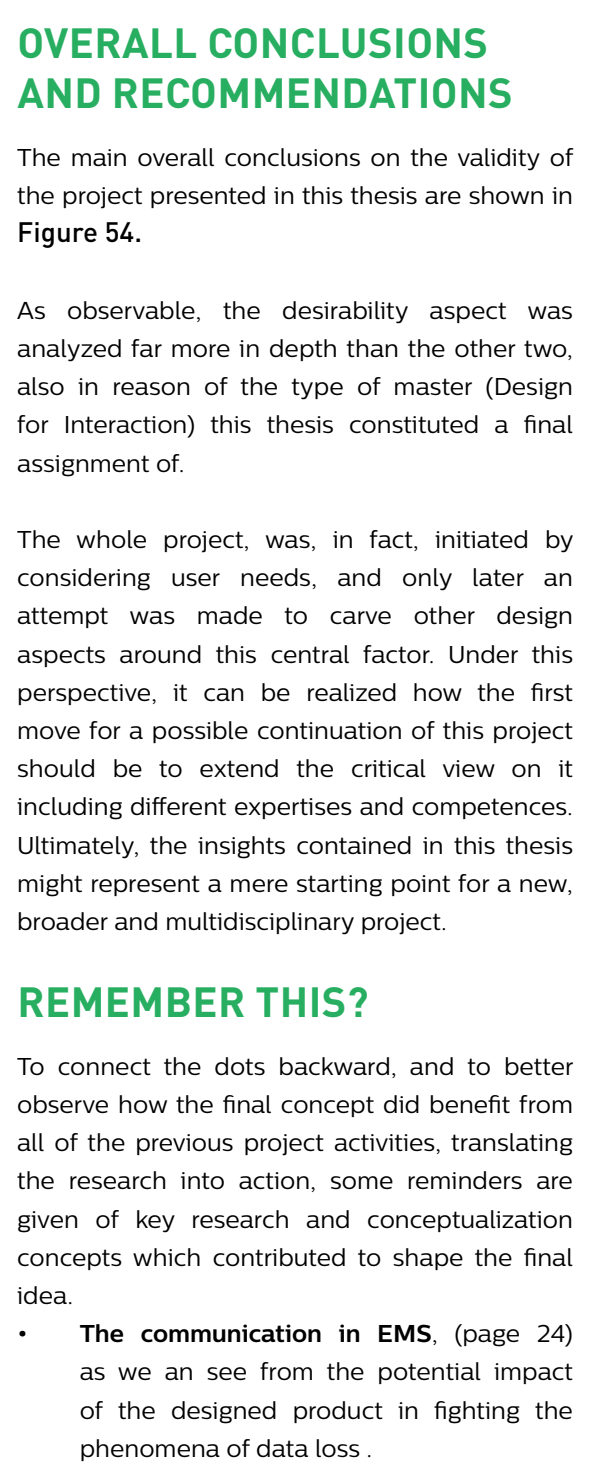

The teamwork in EMS, (page 26) as we can see by how the final idea was designed strategies

The trends and directions, (page 28) tha were deeply reflected in the final viability strategy.

The nurse character, (page 51) as we can reaching character goals such as 'offering support to the ones who need it the most wishes such as faster and more flexible bureaucracy', or defeating stress sources such as "protocol uncertainties.

The time analysis, (page 55) which indicated the road of cutting on reportin time as a chance for speeding up ambulance rescues

The expenence factors, (page 56) as reshapes the nursing experience on dimensions of responsibility persona relationships and foresight.

The flow model, (page 58) as we can se by the way the final idea constitutes possibility to improve flow drivers such as 'positive feed forward," and to flow facilitators such as 'trust and collaboration The direction

the diretion choosing, (page 78) from collected that heavily infuenced the final concept such as to strive for time saving, simple solutions'"alleviating logistic burdens', to enhance 'smart report fillins decisional aid', and 'easier data transfer'.

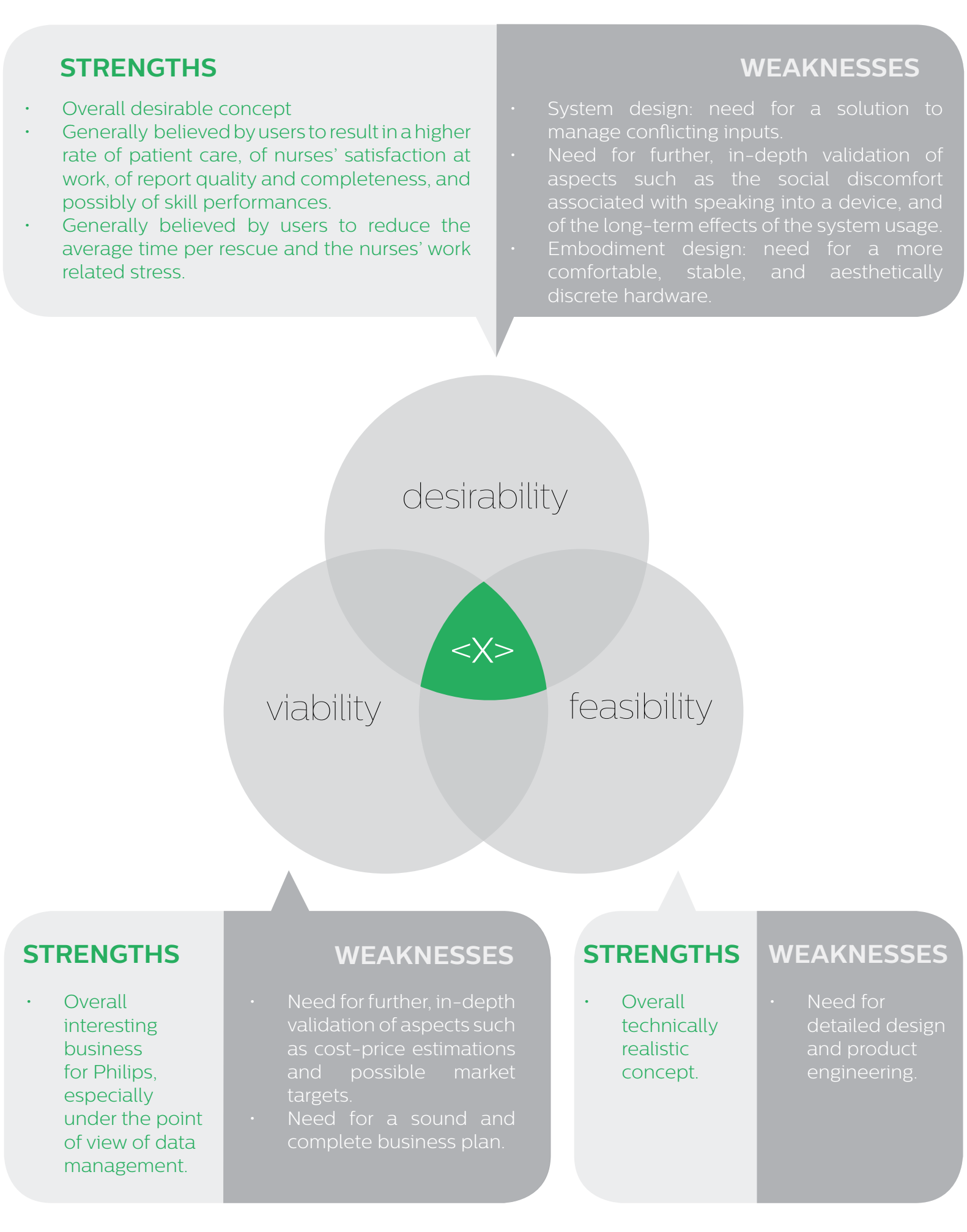




\section{Reflections on research}

could this have been studied better?

RESEARCH STRENGTHS AND LIMITATIONS

In order to better interpret and evaluate the research presented in this project and its results, some points of reflection are provided

Strengths

Overall, points of value of this project's initial phase are to be find in its structure. The different theoretical frameworks (distributed cognition, How) used to shape the researcher view on the
topic before the field research, contributed in creating a multidisciplinary, versatile research landscape. Also, the use of several methods of inquiry (direct observation, online user research, cultural probes, intervews) and the terative and adaptive style given to these very methods heterogeneous but still structured database on the topic Arguably this kind of 'moving target' context exploration style does not perform optimally in terms of time efficiency: in fact, the time spent in conducting the overall research constituted approximately half of the overall project duration.

The usefulness of such a research style, though, was revealed more and more as the project interest kept shifting and deliverables were in preparation, it was easy to 'reshuffe' the content and pick pieces of information orisinally collected for different purposes. A less flexible research style would have rendered this kind of operations difficult, making it impossible for the project to evolve freely
In order for this to happen, however, a rigorous ongoing work of data analysis, documentatio The time rives necessary.

ade this kind of research posstble was, overall, paid back simply wouldn't have allowed for getting to essential parts of the ambulance nursing 'puzzle'

The special relation between nurses and patients (which mainly came from the mos personal and introspective parts of long personal interviews)

The visible effects of a state of high concentration on ambulance nurses observation of a shift).

To this end it is also necessary to mention as a strong point of this project first phase the value of the early involvement of users in the process. Apart from the initial brief, whic provided a starting point, the whole research was directed and shaped by the direct input of the participants, and only a background work of eft to the reseacher. and

\section{Limitations}

Still, many limitations have to be mentioned The most prominch quality and reliability being this a graduation project, it was entirely conducted by a single researcher, with all of the bias and partiality that this can entail. This might have heavily restricted the understanding of the collected data and the translation into structured results, as these actions were all dependent from researchers previous knowledge, mindset and
preconceptions. other words, throughout the whole exploratory phase the researcher could have only be seeing what she expected or was ready to see, without The dimensions of this limitation can perhaps be better understood using two key examples which this constituted a limiting factor. Derng of the researcher attentions or not have been given enough importance to be remembered later.

During the analysis on the wall, pieces of information were inevitably interpreted with a meaning close to the researcher own view. In group projects, conversely this moment ton constives a gredy interpretations and by dong the discover

A second, heavy limitation on the research quality was given by the language factor.

Not being the researcher a Dutch speaker, all of the live interactions with participants from the Netherlands had to be conducted in a language they did not use in their dally life. Although

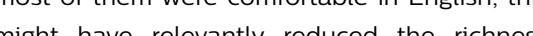
and completeness of the given information Furthermore many direct exchanges on the scene of direct observations -including very elevant ones between nurses and patients, nurses and drivers and generic coffee room talks- were simply missed all together.

Overall, the language barrier made it particularly difficult to reach the more intimate and diminishing the quality for the arswers responding to the research question: "what's on the top of ambulance staff's mind while performing a rescue?

Thirdly, the overall context coverage of the esearch is far from being complete. Especiallly the whole dispatch center system -a crucial part of emergency rescues- was only superficially explored, with no direct observation or specific users involvement.

(estriction - especially be found in its geographical distribution, which could only be physically carried on in the Dutch territory.
Conclusive limitations can be seen in the project's time constraints, in the initial brief sometow restreting the possible scope of the design intervention, and in the expertise focus thesis to be the academic purpose of the Interaction masterpiece.

SUGGESTIONS FOR FURTHER STUDIES

Some recommendations can be provided abou ways to overcome the discussed limitations and
in case of future similar projects.

not in the collection of first-hand data a multidisciplinary group perspective recommendable to minimize the effects of personal bias and specialized knowledge. Native language speakers should be preferably employed to conduct the faceo-face phases of the research. troader and longer coverage should be

inally, if the USA is to be considered a real market target, direct method of research the many possible procedural, legal,

\section{LEARNING POINTS}

The conduction of the research constituted a training field and a source of precious experience learning points taken from this work, divided into research phases, is here fixed for future use

Use of existing theory

Whatever is there to research about, there is very little chance that it was not examined in detail by experts. Spenaing time in discovering and be a priority in the earliest stages of every serious study At the same time, however, the existing theory should serve to the researcher as a starting point for the inquiry, and not as the 
For instance, in this project, the frameworks of distributed cognition shed new the teamwork aspect of the ambulance nursing context, and inspired the researcher
into analyzing collected data under a new into analyzing collected data under a new

Still, it would have been a mistake to blindly keep focusing on distibuted cogntron as the answer to all questions in emergency rescuing since the only aspect covered by distributed cognition is the cognitive one

Many more aspects of the project (the technical, the economical, the interaction, the logistic, etc.) had to be clanfied before coming to a satisfyingly clear picture of the context. A danger faced by many design projects is to transforma other sciences) into tangible products without blending the theory into the vivid complexity of the targeted context.

\section{Research planning}

When defining the research aim and specifically when formulating research goals, diverging and expanding the questions set is useful until a certain limit. Too many questions covering all of the possible context implications could end up not meaning much in ther togeh could ess. Even if context it is not use to schedre how to everything (which is formulating a deailed research plan) from the beginning.

Creating a flexible set of research questions which allows for expansions and unexpected discoveries seem to be, all in all, both an easier and a more efficient way to prompt the exploration. of course, his choce must be also accompanied by surifient practire

\section{Research execution}

The main learning points concerning the recruitment and management of participants are available in Appendix I.

\section{Research finalization}

Fnally, a hard-learned lesson gained during

his project is to know when to stop the

of the scheduled activities were performed

expected, or when new interesting discoveries come up which could prompt new research activites. In this project, six booklets were sent

Collecting all of them back in a reasonable ime before moving on with the data analyss time before moving on with the data analys
was soon discovered to be impossible, mainly because of participants different schedules and obligations or because of pla unresponsiveness

\section{A deliberate choice of the researcher was then} to proceed with the analysis and to try and
integrate the 'new' booklets in the projects at whatever stage they would have finally come in

This not only enabled a more efficient project management, but permitted relevant user input
to be collected in the early conceptualization phase as well-in other words the contact with the users was not stopped after the end of the research phase, but kept flowing in as the project advanced, providing valuable insights and ongoing confirmations.

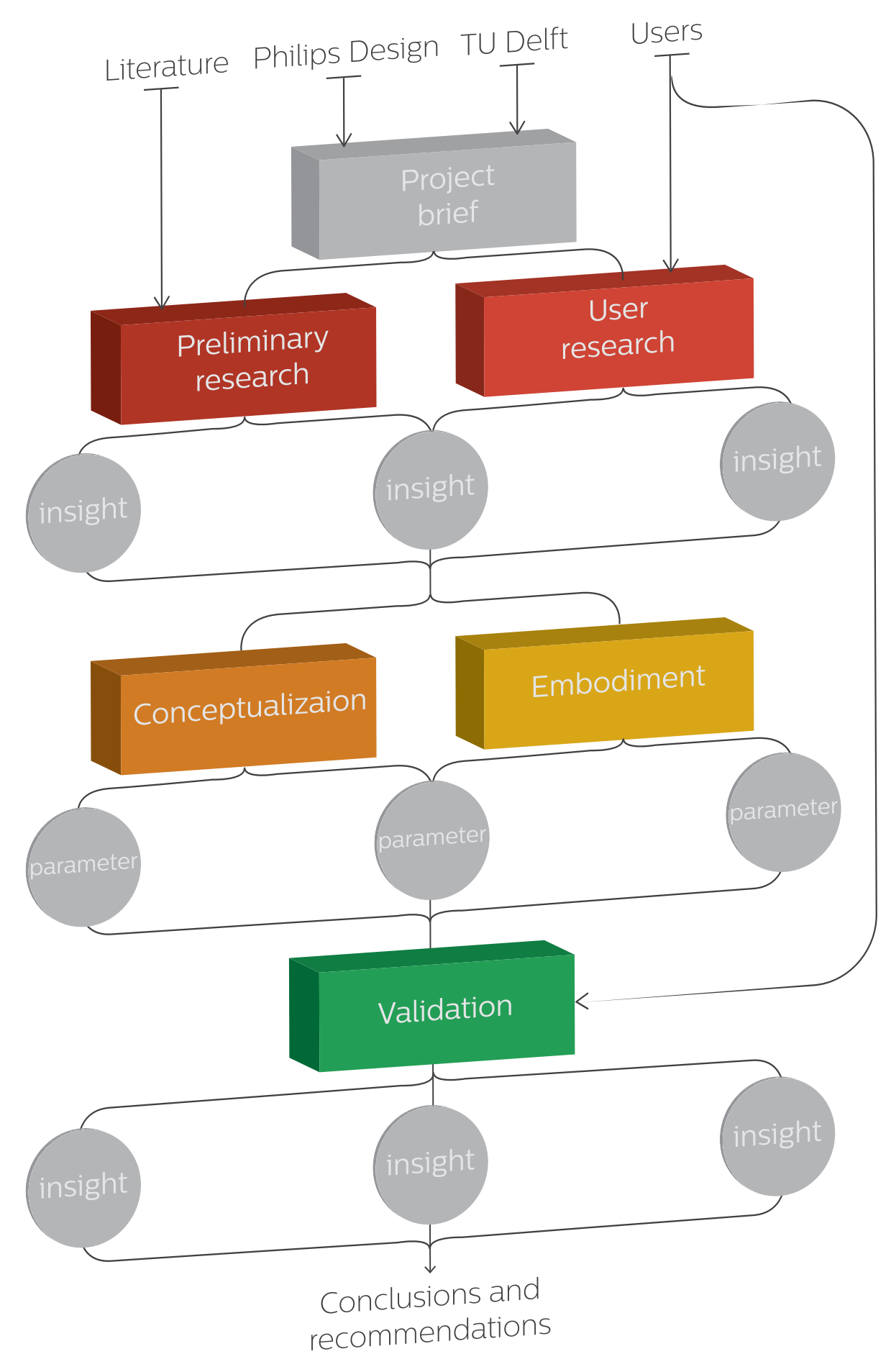

Figure 55 Recap of the overall
design process 
Reflections on

\section{conceptualization}

could this have been imagined better?

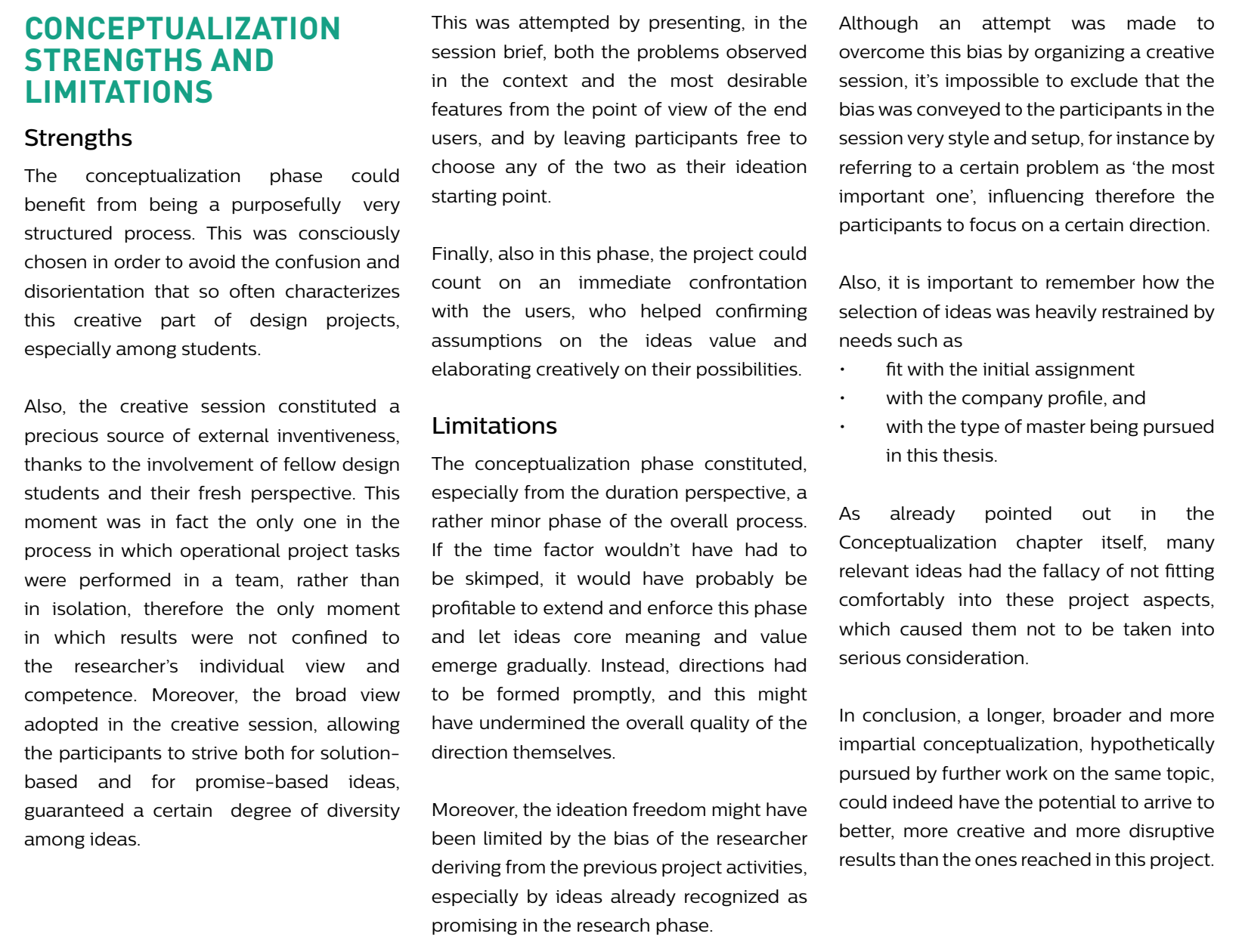

\section{Reflections on validation}

Could this have been demonstrated better?

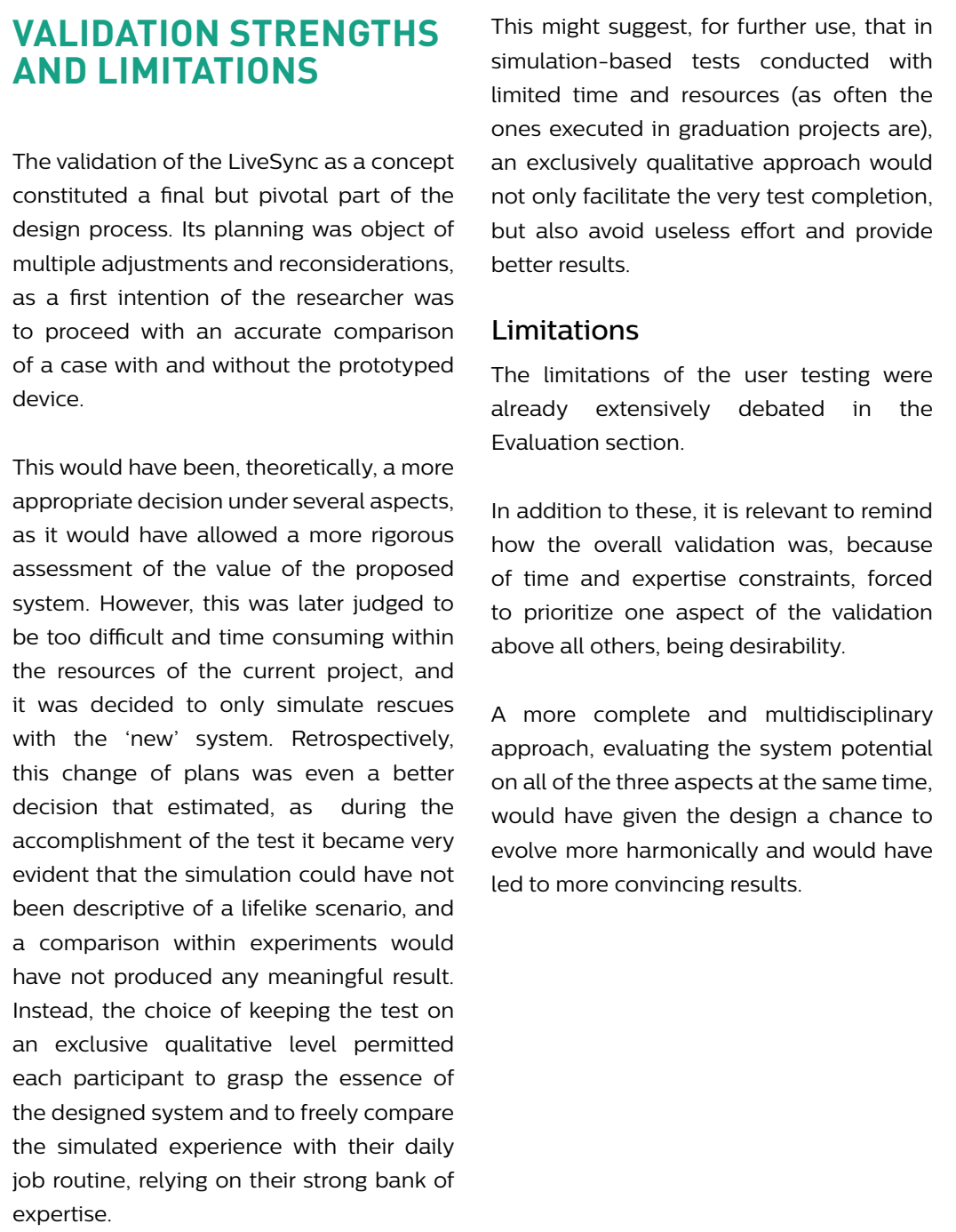

VALIDATION STRENGTHS

The validation of the LiveSync as a concept

multiple adiustments and reconsiderations

as a first intmion or he researcher whs

device

testing were Evaluation section.

expertise 


\section{Reflections on overall process}

Stepping back to see the big picture

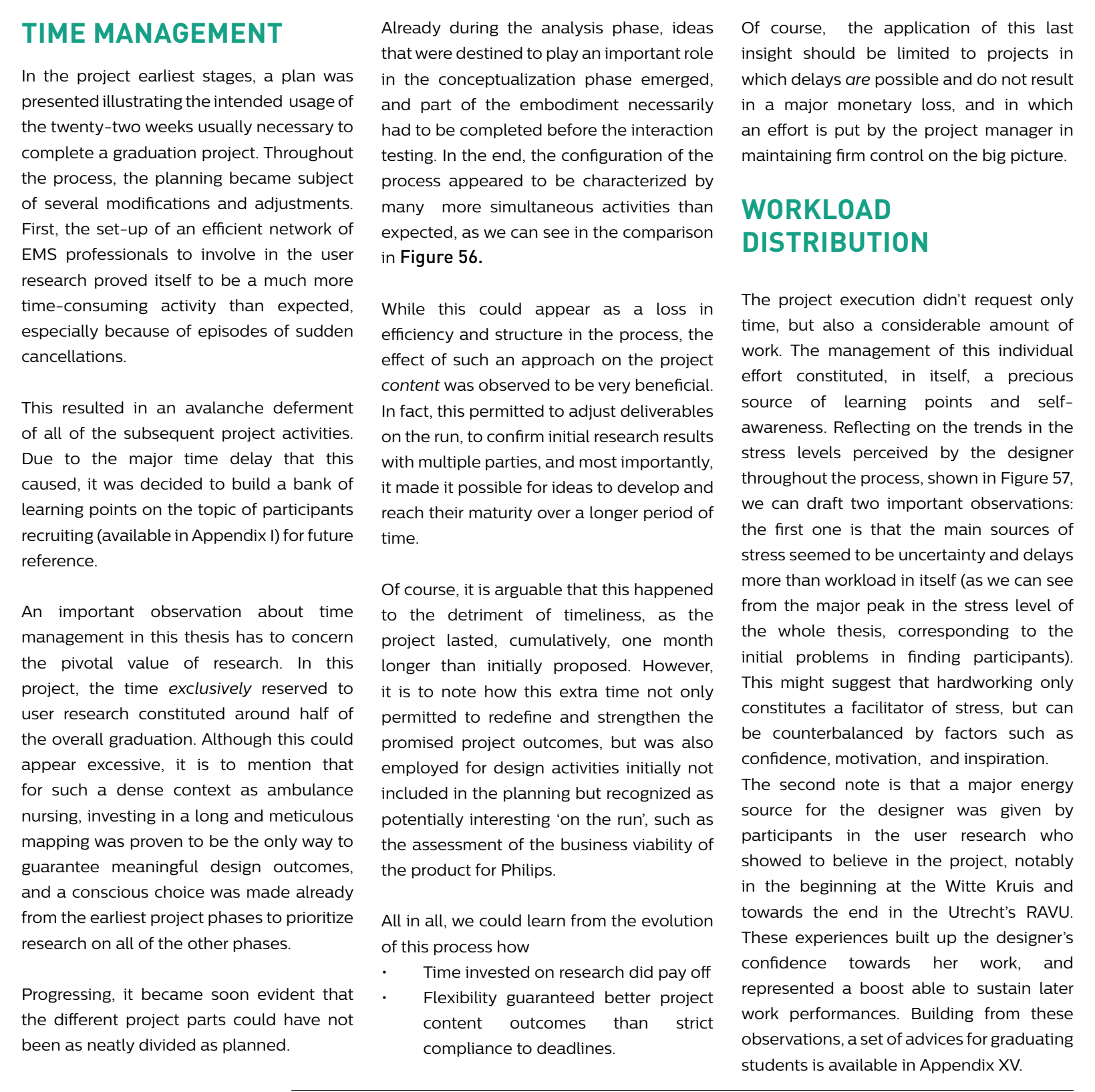

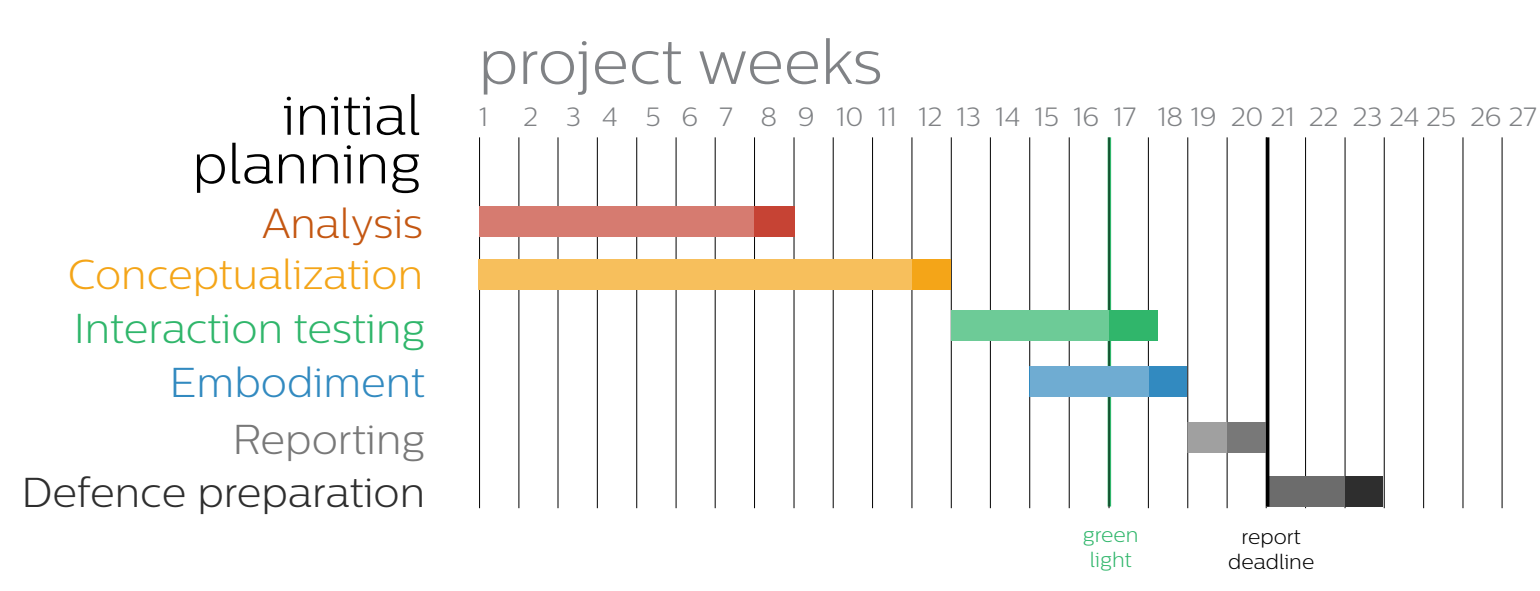
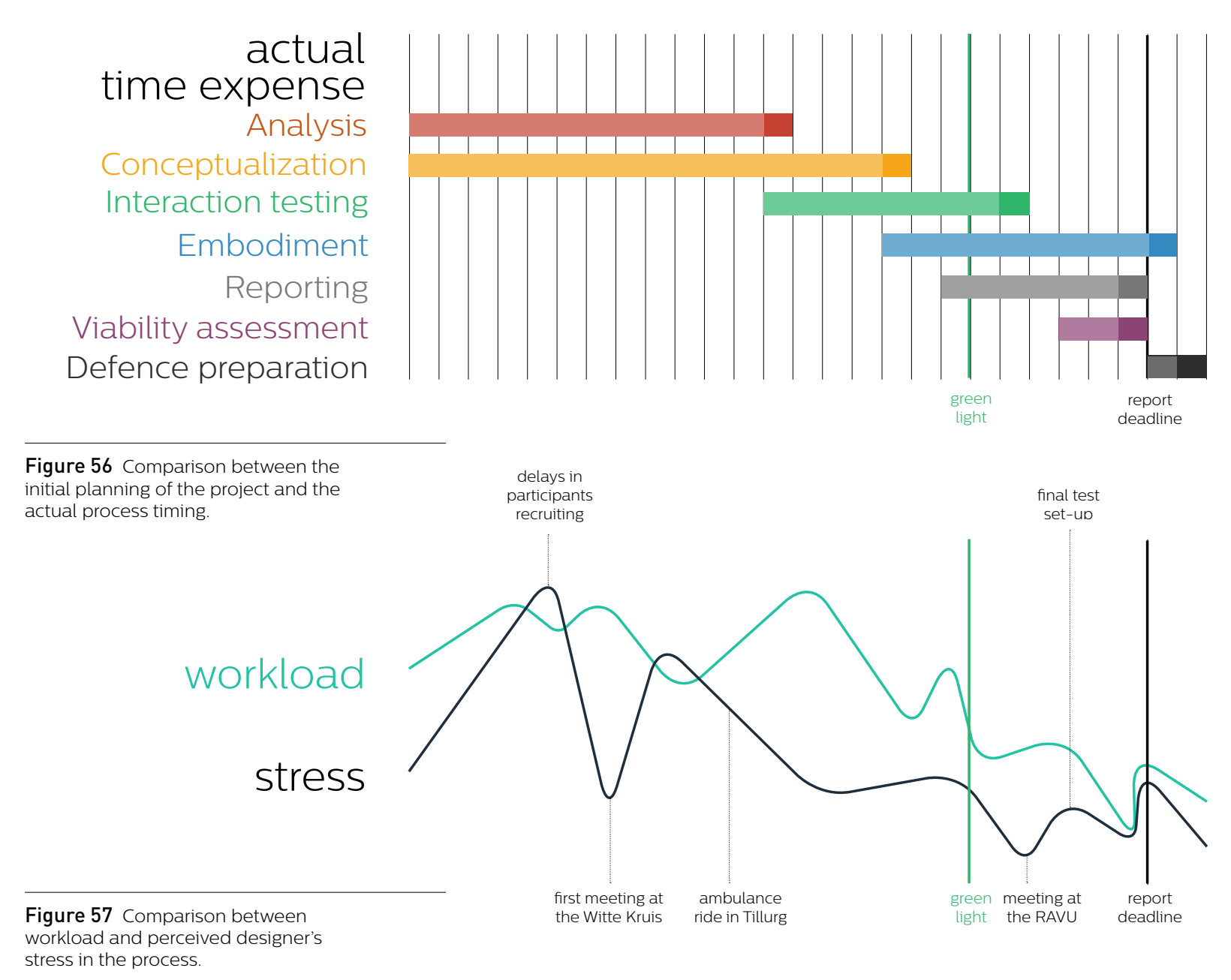


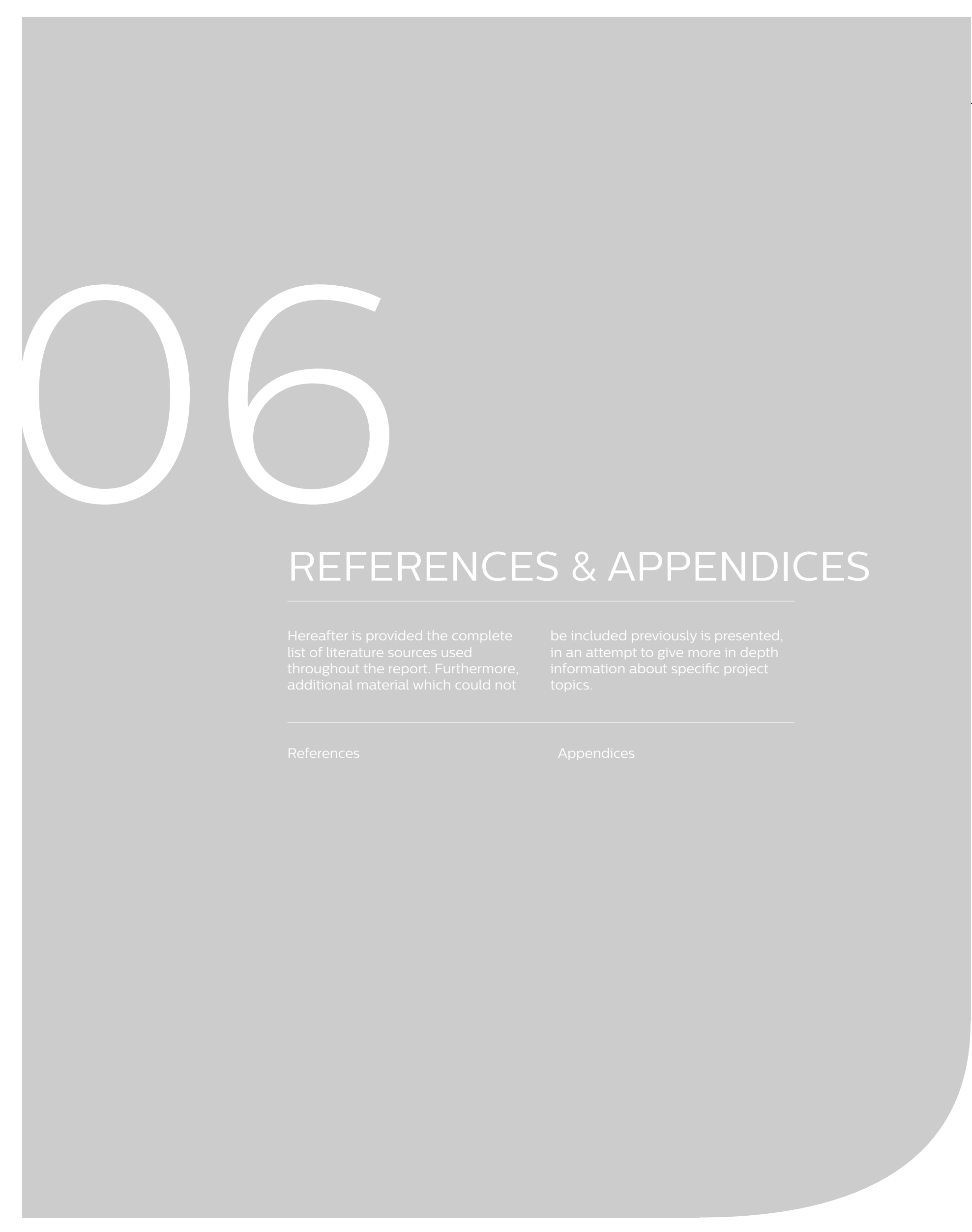




\section{References}

Artman, H., \& Garbis, C. (1998). Team communication and coordination as Cognitive Ergonomics (pp. 151-156)
Contrited conition. In 9 th Conf

Artman, Y. W. (1999). "Distributed Cognition in an Emergency Co-ordination Center." Cognition, Technology \& Work

Bernstein, J. A. (2012). 2012 Invention awards: augmented reality contact lenses Popular Science.

Csikszentmihalyi, M. (1990) The psychology of op
York: Harper \& Row.

sikszentminalyi, M., \& LeFevre, (1989). Optimal expentence in work and pisure. Journal of person

Consumersearch. 2016. Dragon NaturallySpeaking 12 Prem htro./Mww consumersearch.com/voice-recognitionsoftware/dragon-naturallyspeaking- $2-$
premium. [Accessed 26 August 2016]. premium. [Accessed 26 August 2016]

Dean, Joel. "Pricing policies for new products. Hanvard Business Review 54.6

Dick WF (2003). "Anglo-American vs. Franco-German emergency medical services system". Prehospital and Disaster
Medicine 18 (1): 29-35: discussion 35-7 dol:10.1017//1049023×00000650. PMID 14694898.

Dominick Shelton, P. S. (2016) Avallability of ambulance patient care Quality improvement reports 5.
Dutch Ambulance service UMCG Csikszentminalyi, M. (2014) Ambulancezorg leads the introduction The concept of flow. In Flow and the RDTs Tempus Pro | Remote Diagnostic Foundations of Positive Psychology (pp Technologies Limited. 2016. Dutch 239-263). Springer Netherlands

Ulance service UMCG Ambulancezor NHTSA (2015). What Is EMS Remote Diagnostic Technologies Limited. [ONLINE] Available at. http://Www.ems.gov/
ONLINE] Available at: htto:/ wwwrdttld. whatisEMS.htm. [Accessed 09 March 16].

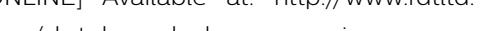
com/dutch-ambulance-service-umc-

Furniss, D., \& Blandford, A. (2006) Understanding emergency medica aspatch in terms of distributed cognition

Grube, A., Schroer, J., Hentzschel, C., \& method: An efficient measure of experience-based job satisfaction. Journal sychology, 81(4), 669-689.

Inverse. 2016. Future Apple eadphones May Use Bone Conduction IOMmprove iPhone Call Quality I Inverse hversecom/article/16640-future-appleeadphones-may-use-bone-conductiono-improve-iphone-call-quality. [Access 25 August 2016

Knowles, H. S. (1949). Bone conduction unit: Google Patents.

Law, E. L.-C., van Schaik, P., \& Roto (2014). Attudes towards user experien uman-Computer Studies, 72(6), 526-541
Osterwalder, A., \& Pigneur, Y. (2010) Business model generation: a handbook for visionaries, game changers, and

Over Ambulancezorg Nederland 2016. Introductie. [ONLINE] Available at https://www.ambulancezorg.nl/. [Accessed 17 March 2016

Philips. 2016. Innovating across the health continuum. [ONLINE] Available innovationmatters/blog/Innovating[Accessed 22 August 2016].

Philips Hospital to Home: Redefining 2014

Kommer GJ. (2010). Hoe is de ambulancezorg georganiseerd? In Nationaal Kompas Volksezondhe Bilthoven: RIVM, - sittp.//wmw nationaalkompas nl> Nationaal Kompas Volkssezondheid ZorglAcute zors Ambulancezorg, 8 december 2010.

Resnick, Lauren B. (Ed); Levine, John M. (Ed); Teasley, Stephanie D. (Ed), (1991) (Dp 1-20) Washinton DC uS Amerion Psychological Association, US: American http///dx doi org/101037/10096-018
Sanders, L., \& Stappers, P. J. (2012). Convivial design toolbox: generative research for the front end of design: BIS.

Stevens, K. M., \& Hemenway, W. G.

(1970). Beethoven's deafness. JAMA, 213(3).

434-437.

Taylor, R. H., Menciassi, A., Fichtinger, G., \& Dario, P. (2008). Medical robotics and handbook of robotics (pp. 1199-1222). Springer Berlin Heidelbers

TED.com $2016 \quad$ Minaty Csikszentmihalyi: Flow, the secret to happiness I TED Talk | TED.com.
ONLINE] Available at: http://www.ted [ONLINE] Available at: http://www.ted.
com/talks/mihaly_csikszentmihalyi com/talks/mihaly_csikszentmihalyi_-
on_flow?utm_campaign=social\&utm_ medium =referral\&utm_source=facebook comsutm_content=talk\&utm_term=socialscience\#t-850497. [Accessed 27 June 2016].

Thüring, M., \& Mahlke, S. (2007). Usability, aesthetics and emotions in human-technology interaction. International Journal of Psychology,42, 253-264

Van Doorn, F., Gielen, M., \& Stappers, P. J. (2014A). Children as co-researchers: More than just a roleplay. Paper presented at the IDC'14: Proceedings of the 2014 conference on interaction design and children, 17-20 juni 2014, Aarhus, Denmark.

van Doorn, F., Gielen, M., \& Stappers, P.J. (2014B). Involving children and elderly the development of new design concepts

Wang, Henry E., et al. "National Services responses in the United States. Prehospital emergency care 17.1 (2013): 8-14,
Webster, J., Trevino, L. K. \& Ryan . L (193). The dimensionality and correlates Computers in human behavior, 9(4), 411(applications of humanSchere Sciences, 92(22), 10011-10016.

Wi-Fi Platform Sends Lifesaving Data Hospitals. [ONLINE] http://www.govtech.com/ Dealth/Wi-FI-Platform-Sends-LifesavingAugust 2016].

Wilson, S., Randell, R. Galliers J. \& Woodward, P. (2009, September) (2) In European Conference on Cognitive Ergonomics (Vol. 30).

WIRED UK. 2016. Google Glass to une vibration instead of traditional headphones I WIRED UK. [ONLINE] (a)

Zaborowski M Kopański, Z \& Leszczyński, P. (2015). Ergonomics at work in he ambulance-the opinion of paramedics. JOURNAL OF PUBLIC HEALTH, NURSING AND MEDICAL RESCUE, 158(2015_3), 27-32. 


\section{Appendix I}

Decalogue for participants recruitment in the medical field.

\section{Appendix II}

List of questions and answers from Quora.com

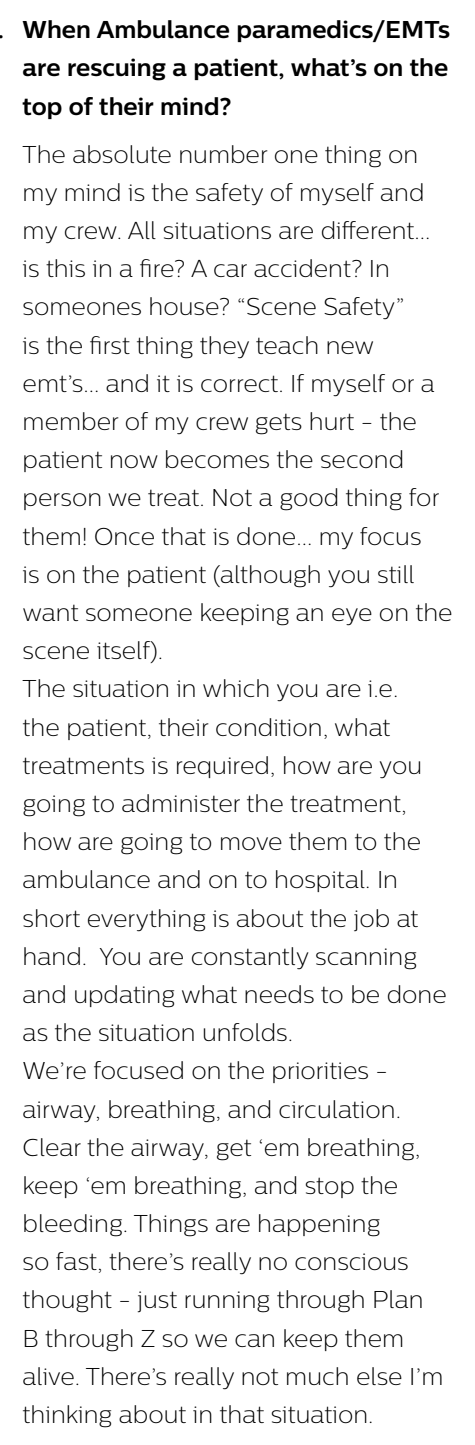

9. Don't expect participants to decide their favorite method of research. had busy schedules, tried to be very flexible and open about the data gathering methods. I proposed different options (booklets asked them choose. This ended up confusing them. Being flexible is important, but selecting the research tools is part of the researchers job. 10. When contacting hospitals: if you have any other option, don't talk to che person usually managing the is to select medicine and nursing school students for internships and stages, and they'll be misled by any other request. Mostly, they'll end up refusing your 'application' (even though it was not one) and you will at. Hype us avoid that if your state interviews,focus groups) and have to put a cross on that hospital.

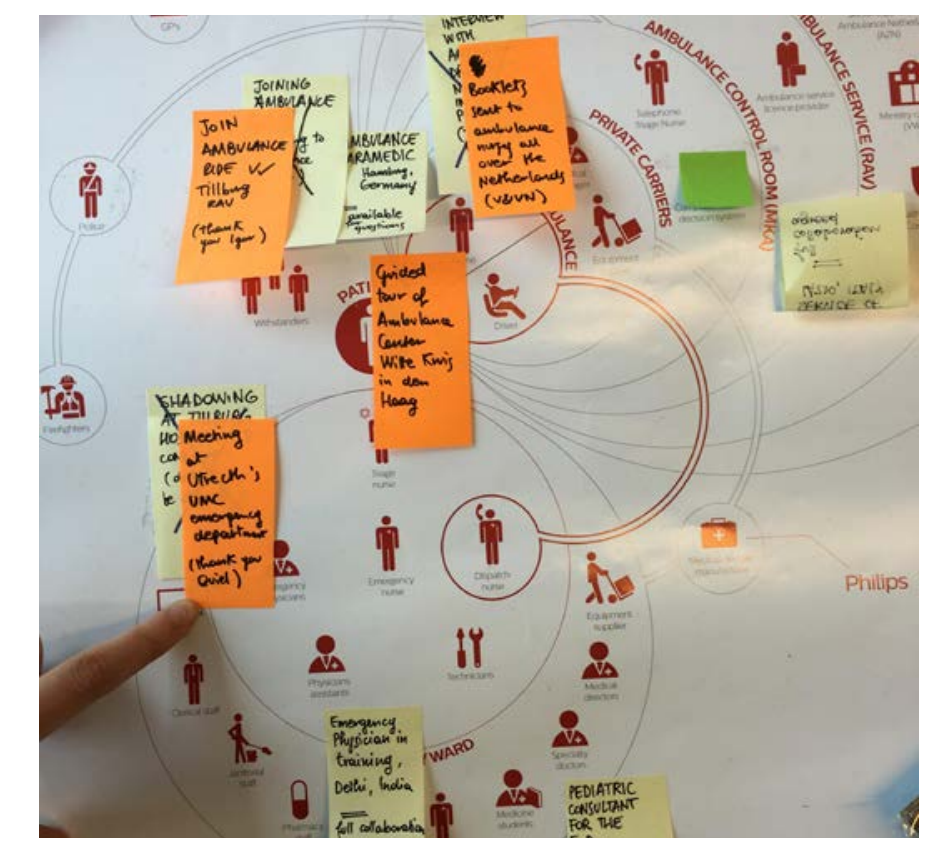
wholeheartedly. Other big fears may
2. What's the main fear of Ambulance $\quad$ 3. How long does it take for paramedics/EMTs when on a

rescue?

First would be finding your family s your patient. Second would be inding your patient is a child. include: Getting stuck with a dirty needle, being exposed to a really virulent contagion, or, my own fear, job.

patient The most coming the next happens is that someone crashes. into the accident we are working does not have a "move over law" ( essentially a traffic law that says when you come upon a scene with flashing reduce your speed by 20 mpht then advocate for one or at least do it yourself: The $\# 1$ cause of line of duty deaths for first responders is traffic accidents and the number 1 type of those accidents is people crashing into existing emergencies.

To add a bit of humor to the answers here: That I will rip the crotch of my palf eaten dinner that t feft on the counter to respond to a call will be eaten by someone else before t get back. That I forgot to close the bay doors as I respond to a call, and all of my belongings will come up missing
rrom the station

\section{Ambulance paramedics and EMTs to} report a rescue?

The treating medic/EMT usually during the shift where the treatment occurs, typically right after the call. Occasionallytitis extremely busy or a few days plicated call and it takes a the company can thistrative side of before details are available.

complete by end of shift. and it may take an agency anywhere from 24 hours to a month to process the report, though there is a financial incentive to process it as quickly as possible 
4. How much time do ambulance how much is for reporting?

In general, paramedics spend the ime with the patient the entire contact and transport. We don't load them and hop in the front passenge seat. Someone is always supposed to be in the back with the patient most things in EMS is "it Depe Factors such as simple logistics paper vs. electronic charts, softw programs, and time of day all play a ole. In my system, we have a goal for 30 minutes for a BLS chart, and 45 minutes for an ALS chart, though I an a slow charter so I seldom beat those marks. But my charts are always worth the trade off In addition a very complex call could take $1-2$ huts to chart depending That would be unacceptable or unsustainable in some volume driven systems, but t works for us. On the positive side, charting can be a team experience. The lead types the narrative and assessment, while his partner fills in alt the data sets and demographics whe spends their time face down in a computer writing the chart and looks up only to ask a question. Certainly in busy systems there is a LT of pressure to keep up on your paperwork to be available for calls. that said, I have taken the computer away from students and forced the to talk to their patients like peop make them ask small tak quest revisit their assessments, or anythin other than hiding behind the charting Call me old school. but I come from the pre-digital era of paramedic where treating your patients like people was important, and you only charted when done.

Face to face time with the patien is usually as little time as possible because fif you are busy shooting Io take another call and let's face it. f someone calls for an ambulance is in the best interest of everyon possible if they need to go. For most transports, once the patient is stabilized and transport begins its a simple matter of fust keeping during the ride which affords you the chance to complete most if not all of your paperwork before you get to the hospital. That way you can get the signature that you dropped off the patient and transferred care to the ER, rip off their copy, and be on your merry way. On calls that require ghts and stren transport though, the scribbling down vitals on a notep For those calls or the calls that nvolve law enforcement or shady circumstances, it typically ends being a good 20-30m+ of novella writing after the ER has your patient The one who was doing patient care will typically do the paperwork while the other medic will clean and

boxes.

done but I don't think has can be Iudged fairly either I have been on simple calls that took me 10 minutes to write a report but a couple hours or more with patient due to circumstances. I've also had times Where Ihad 15 minutes with the patent and an hour or more on the On a typical call, my goal is to spend no more than 20 minutes on scene. I have a transport time of 8-12 minutes depending on where the call is and which of two local hospitals I am transporting to. That gives me an average of 30 minutes with a patient. At the hospital, my goal is to complete patienthand over and complete my ran form in 40-45

\section{How much do ambulance paramedic}

station - some medics might only

- big city medics might make 25-28 was a medic on the bad side of Columbus, $\mathrm{OH}$ and ran 25-28 times disability. It's a young person's job. Just lifting that weight every day in awkward positions is going to lead to back problems, shoulder issues, and more. Add in contamination, PTSD etc. etc. etc., and it does just not make for a long career

one 12 hour night shist with and night shift every other week So in effect, I work a 36 hour shift get a night off and work another 12 ho day shift one week, and the next week, work a 36 hour shift, get a night off and work a 24 hour shift. I work in a rural service that averages a couple calls a day, so I usually have some last about 2 hours since the nearest hospital is 30 miles away. There are weeks when we run constantly, and those are harder as sleep can be hard to get. Mentally and physically, I have not had any problems with working this schedule. My schedule ends up being 3 days on, 3 days off, then 3 days on and 5 days off. I like the way (is and would hate to see it changed ram an Emt and work wish

hour shifts with usually just 30 minutes off. It's not for everyone.

think it is sustainable but the thought of leaving for an easier line of work is inevitable. Even so, it's nice to have real stakes, real risks in your life. A person without struggle, without a reason to strive to preform their healthcare worke thoron. As a hurts to take a little time off to catch your breath and let the fire to help thers rekindle inside you Weally dependas whe working, and also what sort of emergencies you are attending to eel that yes it is of course mentally hable Obviously again this wil pend on the person we are talkin

unt of hours worked all for The department I work for runs ot of calls. If your on an ambulance or the rescue truck you will run all day with sleep deprivation
What are ambulance nurses most
frequent causes of disciplinary

Strangely enough the greates number of complaints agains Paramedic's are conduct related. Most of the time once investigated the cause is found to be misresponses to a tense situation, or mis-communication on a scene very occasionally there are complaints lodged about the care/treatment provided by the paramedic and those typically involve extren cases of incompetence. Your yypical paramedic is a well trained, competent, compassionate provider of meaical/trauma care in emergen discipline within a

end to hover around $1 \%$. So as a member of the public you have no cause to worry that there are rogue Paramedic's responding to your calls for assistance.

Many paramedics and EMTs especially new ones, have one majo alsciplinary concent. paperwork at least wis pivate a a big issue, companies and a lot of it has to do with medics omitting details by mistake. Much of the time medics EMTs are running a lot of calls and are trying to write their reports in the most efficient ways possible, so many newer medics forget detalls that could be important when witng ther report. Supervisors about paperwork that either wasn submitted properly or is missing something that could be considered crucial to the case at hand. For example, if a run number was written wrong, it could affect finding it later if a court case comes up. If you as a Paramedic miss an mportant detail in your narrative, you may also have against a lawyer who's questioning for patient care, a lot of times the supervisor or other person examin paperwork will often reprimand a Paramedic or EMT for missing details on their report of the incident They want to protect their job as well as the companies reputation because disciplinemselves, they have to the time it is an eloyee. Most of issue that hapens mo clable than it should and supervisors have to do their iob in disciplining their employees that make the repetitive parties involved

There really isn't one primary thing that medics get disciplined over as a whole.tr really ends up being a trend of things that some people get people don't clean and restock the procedures), and for some it's the driving behavior. If there was one single thing that tends to hit more Granted, sometimes you get hit by that Gam call that doesn't allow you horoughly clean and restock but that usually only happens $2-3$ times a month. When it really gets to be a constant habit though and the last call was hours ago and people decide to sleep until 6:45 and THEN iry to clean and restockin $15 m$ every and come the the gets pissed You wouldn't else's job every time for them. The biggest key is to take the extra 10-15 minutes after each call and make sure everything is squared away and in a 
the truck when you clock in. 'Ill hings happen from time to time. but when you find yourself having to spend $45 \mathrm{~m}$ or more first thing in the morning organizing, restocking, and cleaning over and over agan, hiats have that 7:07am call that comes in before you have the chance to go. hrough your supplies and on-scen sn't the place to discover that you don't have XYZ, like not even a single $\checkmark$ drip set (the tubing that connects the bag to the patient) atthough the lady shift said everything was good can helieve they got a wrote ro for

trouble Theress a lot of people in you didn't document it, you didn't do it". It's a sad truth, but most of us are taught to document to defen ourselves in court more than to plainly state patient condition and is billing Both privute and is billing. Both private and public
services bill insurance Insurance

companies, especially Medicare/

Medicaid, will do everything possib o pay less than billed or pay nothin off and phrased just right, it turns into a hassle to get them to pay their bills. A medic is just as likely to get his ass chewed about properly care Failing to follow state EMS protocolng to tollow state EMS be an issue. If you are supposed to do something but don't oryou do something not usually done for hat condition, you will hear abou to during a QA report. Every report we do is reviewed and they go by a checkiss to make sure everything matches. Relations with other

Some people just can't get along and will make everyone else on the crew miserable
7. What is a superpower that could their job better?

Clarity of thought

Lie Detection? Seriously. The only people who get lied to more than paramedics are law enforcement. The sad part tis people lie to paramedics afraid we will iudea them whe is probably tree we are human Butit is usually good natured At least we will reserve judgment until we are done taking care of you at least. On the positive note, our threshold for judging you negatively is probably pretty high. Much higner than the average Joe. So, if you get the it is legit Our benchmarks ares are skewed They are afraid we will tell their spouse they are still smoking when they said they should have qu (trust me, they already know). They are afraid to tell us how much they have had to drink (we can guess, though sometimes people impress even us.) I am pretty sure "Two Beers" is not the truth. Why ist always two gong to yell at them for not tar their medications They are afraid to admit they are alcoholics and don't want to admit they drink every day (trust me, we probably already know by looking at your living conditions, mannerisms, physical assessment, and medical history). They think that uf they play up their pain they will get more pain medication. They think about the silliest thes They thin they will get in trouble with the law This is a mixed bag Some things we are required to report to $L E O$. You show up in my box with a pair of little girls underwear, bloody hands, and a smile.... am catling our friends in blue. You show up smoking a little weed, I dont care. And the same better stuff to do So maybe this post is a little bit cynical and a tis pos Can I chose x-ray vision instead? 


\section{Appendix III}

Time line and diary of the observed ambulance shift.
07.30
08.30
09.30
10.30
11.30
1st RESCUE
2nd RESCUE

1st RESCUE (55 min)

08.15

09.10

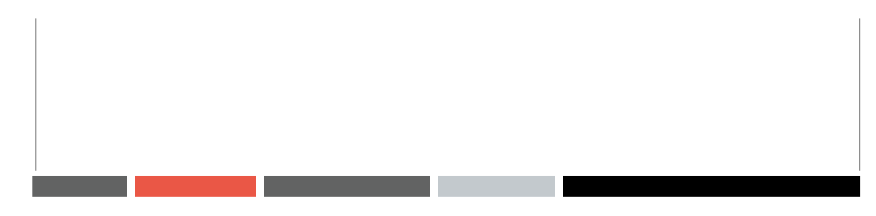

2nd RESCUE (1h 6min)

10.54

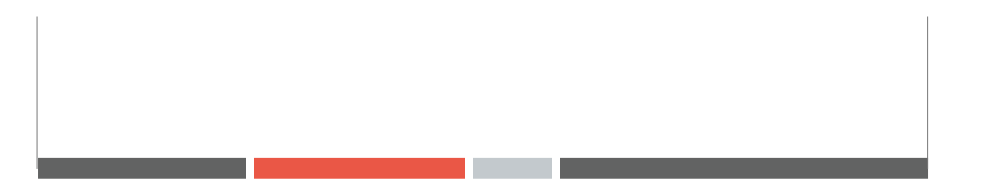

3d RESCUE (54 min)

12.43

4th RESCUE (1h 3min)

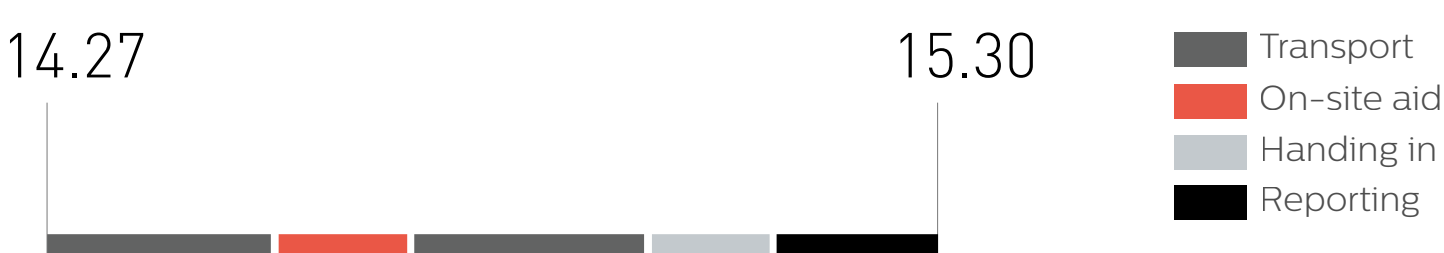

3d RESCUE

1st RESCUE (psychiatric/abdominal pain) $\quad \begin{aligned} & \mathbf{P}=\text { Paramedic } \\ & \mathbf{D}=\text { Drive }\end{aligned}$

1st RESCUE (psychiatric/abdominal pain) $\quad \begin{aligned} & \mathbf{P}=\text { Paramedic } \\ & \mathbf{D}=\text { Drive }\end{aligned}$

Case: Adult man with $\mathrm{A}$.

Procedure: The crew finds the subject on site (street) together with the caller and the police. The subject is lifted on board of the ambulance where first controls are made by $\mathbf{P}$. From these first results, the subject is suspected to suffer from alcohol abuse. The patient is carried to the nearest hospital, while $\mathbf{P}$ asks more detailed questions about his state (medication intake, amount of alcoholic beverages consumed). At the arrival at the hospital, $\mathbf{P}$ gives an extensive update to the receiving nof D refurnishes the ambulance.

\section{2nd RESCUE (psychiatric)}

\section{Level of emergency: Planned transportation}

Case: Adult man with diagnosed psychiatric problems having to be transported in a mental institute.

Procedure: The crew gathers with a police unit, family members and a psychiatrist before attempting to take contact with the patient. The patient is reluctant to join and lock himself home, but slowly the police gains his trust. The patient is laid on the stretcher and transported in the ambulance by $\mathbf{P}$ and $\mathbf{D}$ together. A police officer joins the ambulance for safety reasons, but the patient is silent and quiet and $\mathbf{P}$ fills the administrative paperwork during the ride

At the arrival in the mental institute (still joined by the police unit), $\mathbf{P}$ gives a brief update to the receiving nurse. The ambulance heads back to the center, where no paperwork is needed ( $\mathbf{P}$ already filled it during the ride, plus for planned transportations not many details are required)

\section{3d RESCUE (COPD)}

Case: Adult woman with COPD complaining about respiratory problems

Procedure: The crew arrives at the specified address and has some small difficulty finding the way to the right door When the apartment is found, the crew finds the patient and a family members and briefly assesses the situation. Also, a whten note from the patient General Practitioner is found indicating some patient information and her medication pere

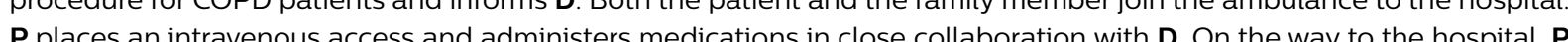
talks with the patient reads the paper note and writes in the laptop at the same time. After the arvival the hospital, the patient is handed in with a brief voice update by P. Back at the hospital center. D cleans and refurnished the ambulance while $\mathbf{P}$ fills in the report: in this case, the filling is slightly longer since oxygen and medications were administered. Also Problems with the system.

\section{4th RESCUE (psychiatric)}

Case: Adult woman with psychiatric problems in a self-harming attack (already showing lacerations from broken glass). Procedure: The crew arrives at the specified address and finds the police on scene. Both P, D and police officers get in the the ambulance on the stretcher. A police officer joins the crew during the ride During the transportation to the hospital the patient starts getting agitated, shouts and tries to break things/rise up against $\mathbf{P}$. The patient is slowly comforted and calmed down again, but keeps showing evident emotive turmoil. At the hospital, $\mathbf{P}$ gives extensive explanations to severa Emergency Room staff members. Back in the center, $\mathbf{P}$ fills in the report while $\mathbf{D}$ refurnishes the ambulance. 


\title{
Appendix IV
}

Cultural probe content (English version).

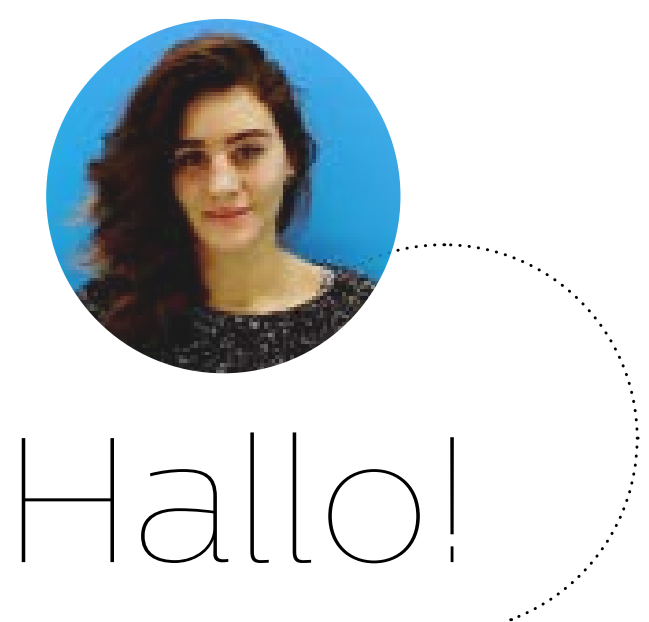

This is a small book about you and your experiences as an ambulance nurse

It will be used to inform, inspire and sensitize a design team working on the topic of easier

interactions with technology in Emergency Care. For more informations about the project, please visit

http://goo.gl/FOU9cw

The book is now only an outline, and needs to be filled with your words, sketches and thoughts in order to become a real one. This won't take long; the very best would be to complete one or two

exercises a day, so that you have time in between to reflect.

There are no right or wrong answers in this book, only 'your answers', or the ones that best reflect your experiences. Because of this, we kindly ask you to fill the booklet on your own. Please try not only to write, but also to draw, cut and paste images as much as possible. We realize some of the questions are more than just professional, but the more stories you can tell us, the more interesting it will be for us!

When the booklet is finished, please contact me and l'll come collecting it at your preferred time and place. We will keep the book for a few weeks, after that you can have it back.

\section{some info}

\author{
profession: \\ years of experience: \\ what I love about my job:
}

Best wishes,

Valeria

Graduate student - Effortless interactions in EMS - TU Delft Design Team

valeria.pannunzio@gmail.com 


\section{What do I do?}

\section{What happens during a call?}

Think about a 'standard' emergency call: what happens in between the notification of an emergency and your return to the break room? What do you do during these stages? Pinpoint the most relevant events on the blank timeline and brifefly describe them (see small example in the next page describing my standard day). If you find it easier, describe the last call you responded to.

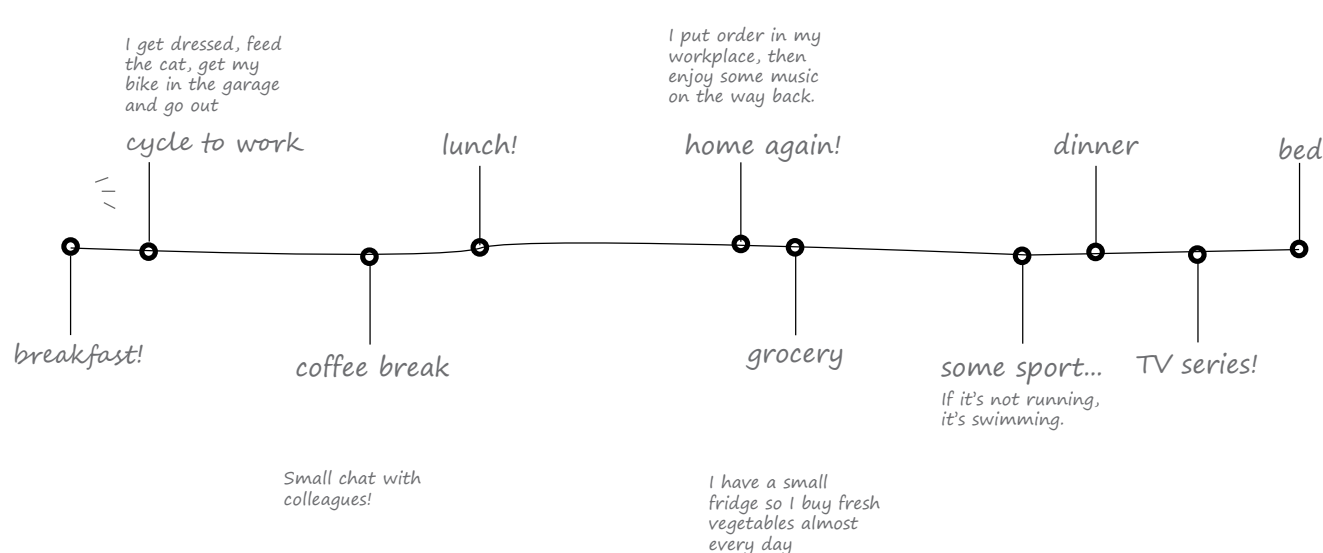

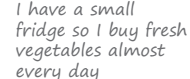

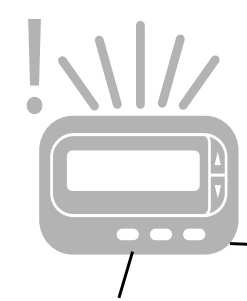

Beeper rings

\subsection{How do you exchange informations with other parties?}

Identify the moments on the timeline in which you have to communicate with

other parties. For each point of communication, paste the corresponding sticker

describing the way communication happens (by pager, walkietalkie, toughbook, in

person, etc). Feel free to use the blank stickers for more channels I didn't think of

Don't worry if the stickers overlap or cover previous information.
What is this?

Thick the box

$\square$ 'Standard' cal

Real call 


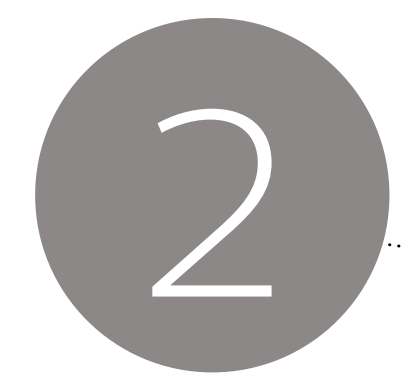

How do I work?

3.1. Now please try to picture yourself as much as possible in a reait situation at work. Everything is happening around you now and here and you're called to act upon it. What's going on in:your mind? Complete the sentence with a quote that

could best describe your way of thinking on an emergency

When I'm on the scene of a rescue, I mainly focus on...

pay attention to

The first thing I do is
When I'm carrying a patient to the hospital I mainly focus on

After I have handed in a patient at the hospital, I mainly focus on.. 


\section{How do I like it?}

\subsection{What are the best parts?}

During which parts of the rescue you feel more in control. efficient: comfortable? Paste these green stickers on the timeline: on exercise one. Don't worry if the stickers overlap or cover previous information. Then, explain briefly why you 1st groene moment eel this way in the spaces beyond.

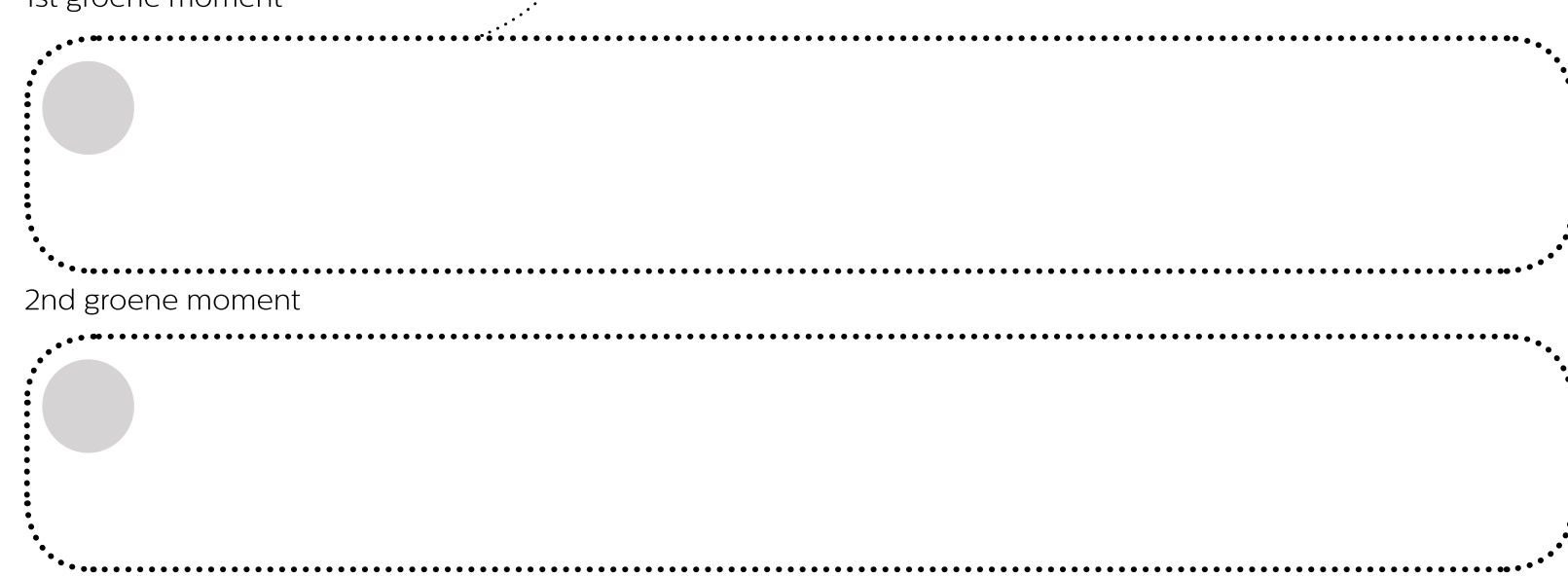

\subsection{What are the worst parts?}

During which parts of the rescue you feel more under pressure, stressed or out of control? Paste these red stickers on the timeline on the previous page. Then, explain briefly why you feel this way in the lines beyond. Don't worry if the 1st rode momen stickers overlap or cover previous information.

3.3. What are the most frequent problems happening during a rescue? Here are some hypothetic problems. In your experience, how frequently do they really happen and how bad do you think they are? Fill in the circles from 0-5 to describe it. Then, write down in the emoty cells other problems you face in your everyday job

\section{How often? How bad it is? $(\mathrm{O}=$ never, $\quad(\mathrm{O}=$ not a real problem.} 5 =every day) 5 =very dangerous)

$\therefore$ Sometimes the equipment doesn't work 000000000

well Sometimes I forget/have doubts about the $\bigcirc \bigcirc \bigcirc ?$ protocols

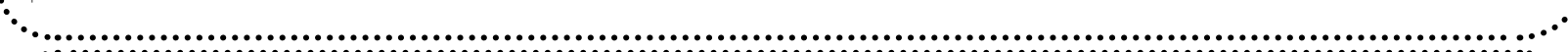
Sometimesl'm unsure about which protocol to follow

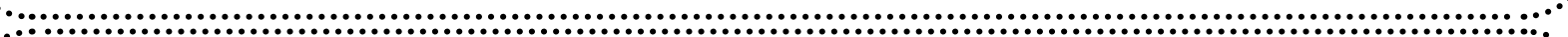
$\therefore$ Sometimes it's difficult to convince a patient $\bigcirc 00000000$ to collaborate

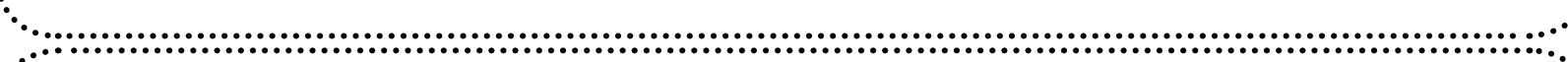
$\therefore$ Sometimes it's difficult to remember details $\bigcirc \bigcirc \bigcirc \bigcirc \bigcirc$ of the call that I have to report

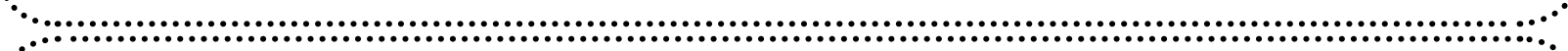
$\therefore$ Sometimes the administrative paperwork $\bigcirc \bigcirc \bigcirc \bigcirc \bigcirc$ takes too long

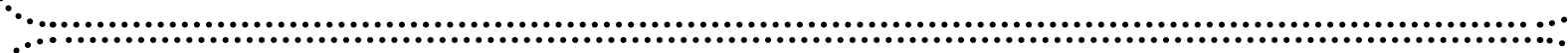
Sometimes it's dificult to communicate with 000000 other parties (MKA, hospital)

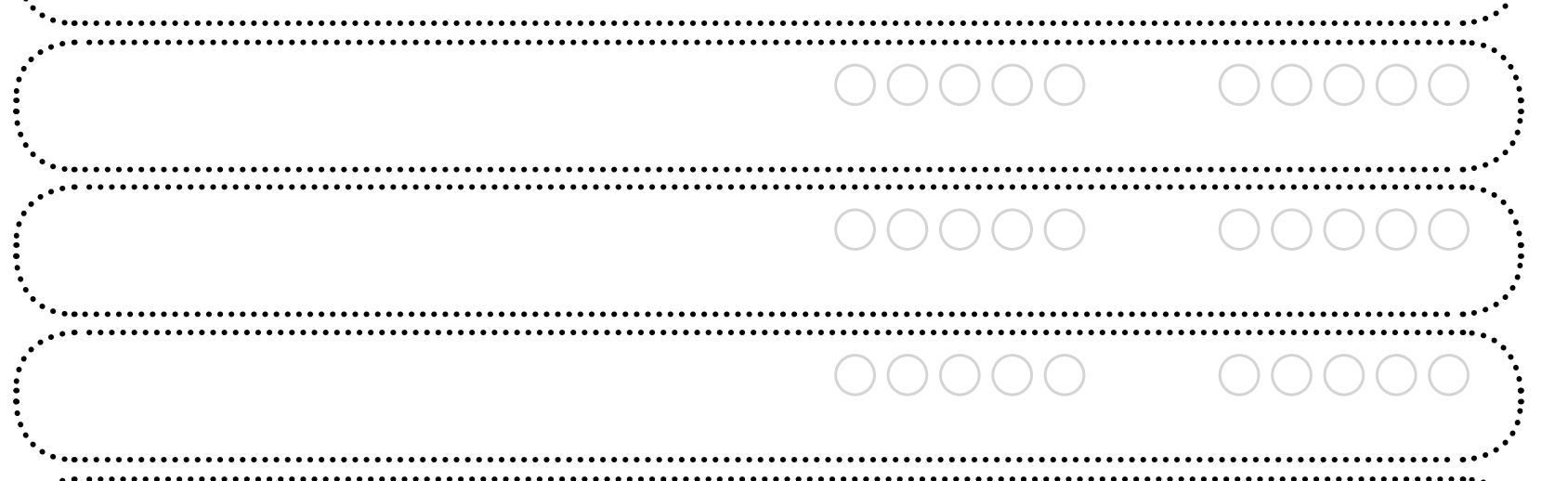

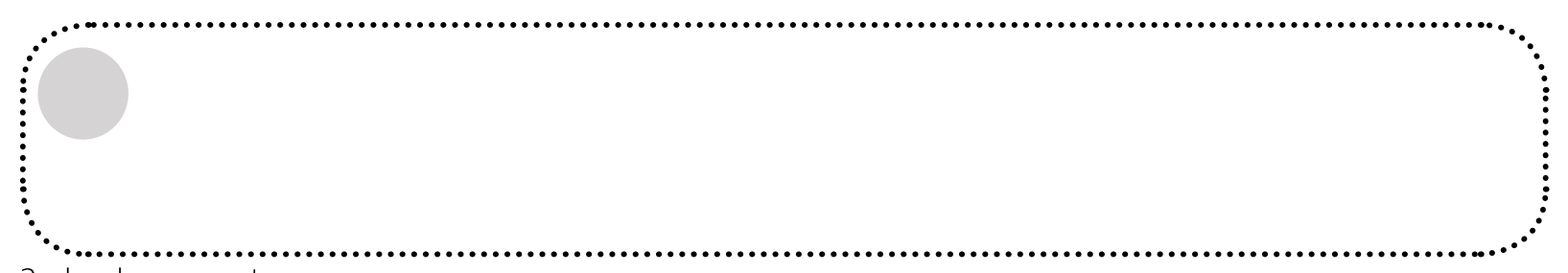
d rode moment

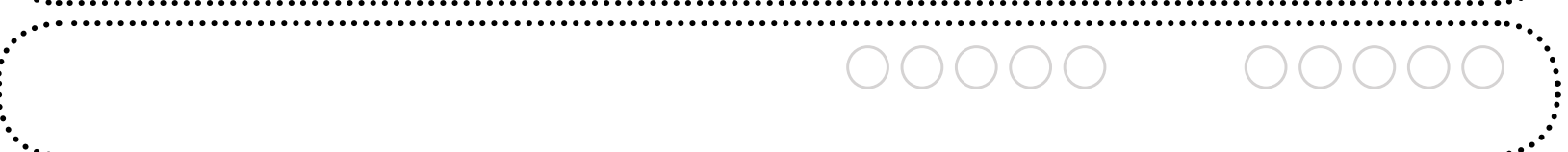


schedule among the examples given, and add more in the

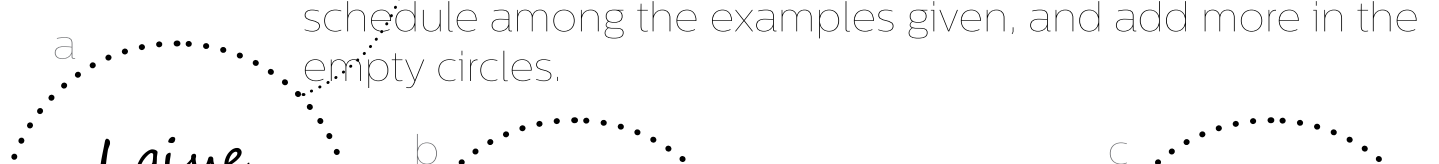

I give treatment<smiles>[CH2][CH2]</smiles>

$\square$ in my daily

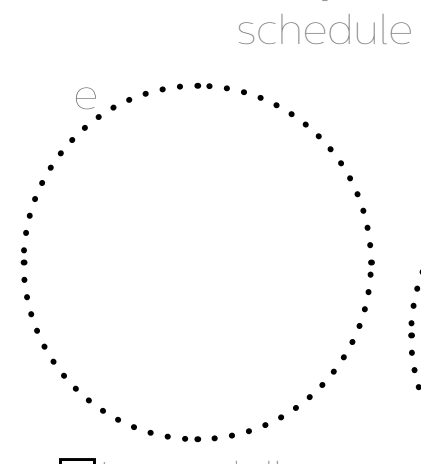

$\square$

schedute

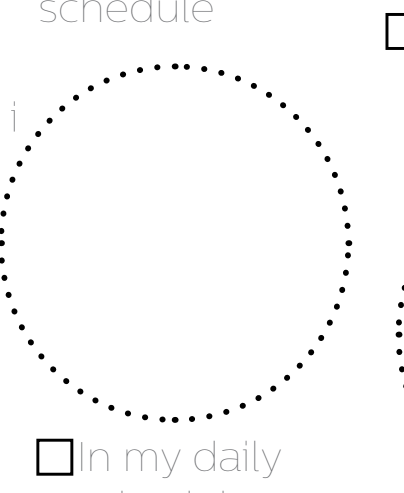

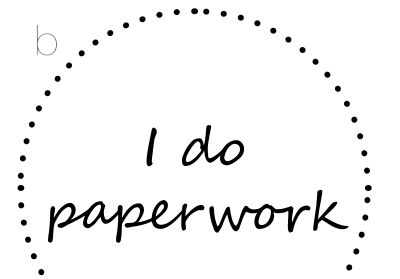

$\because$

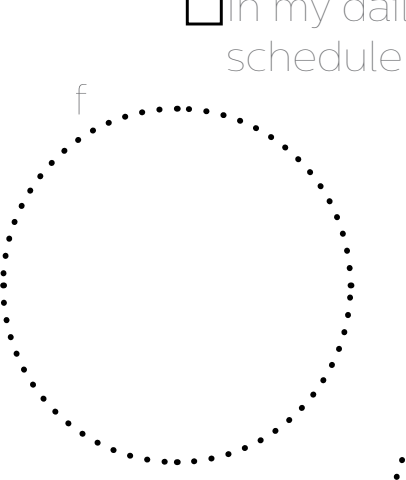

$\square$
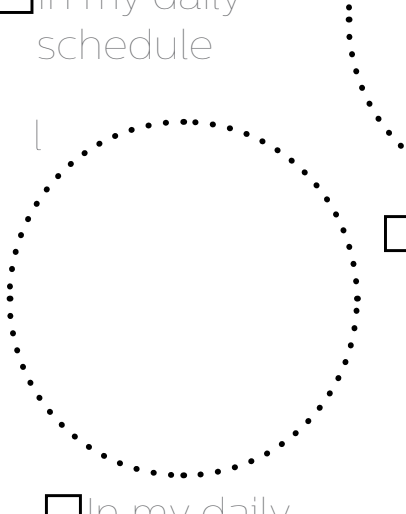

$\square$ in my daily

schedule

\section{I check the} equipment

$\square$ In my daily

schedule
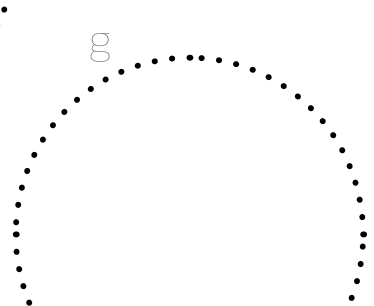

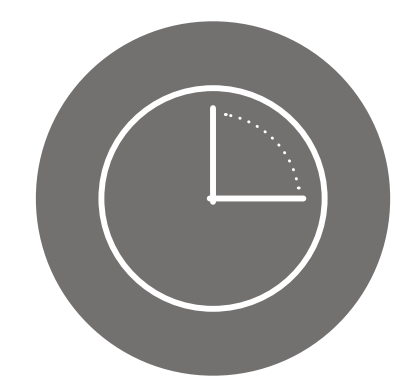

How much of my time does it take?

4.2. How is your time distributed?

Please rate the previous activities on a scale from 1 to 5 ,

in which 1 is 'usually very quick' and 5 is 'usually very time consuming

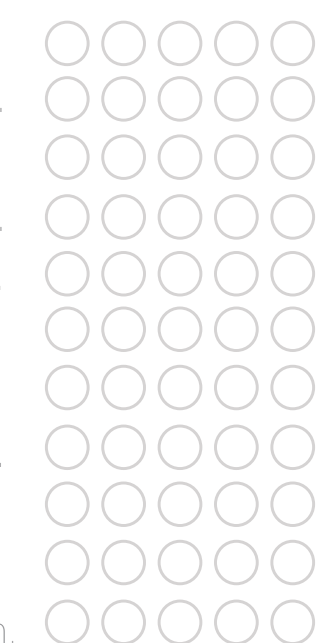

4.2. Is there an activity in the previous page you would prefer to avoid all together?

Just write an X on them! If you want, briefly explain why in the box here beyond 


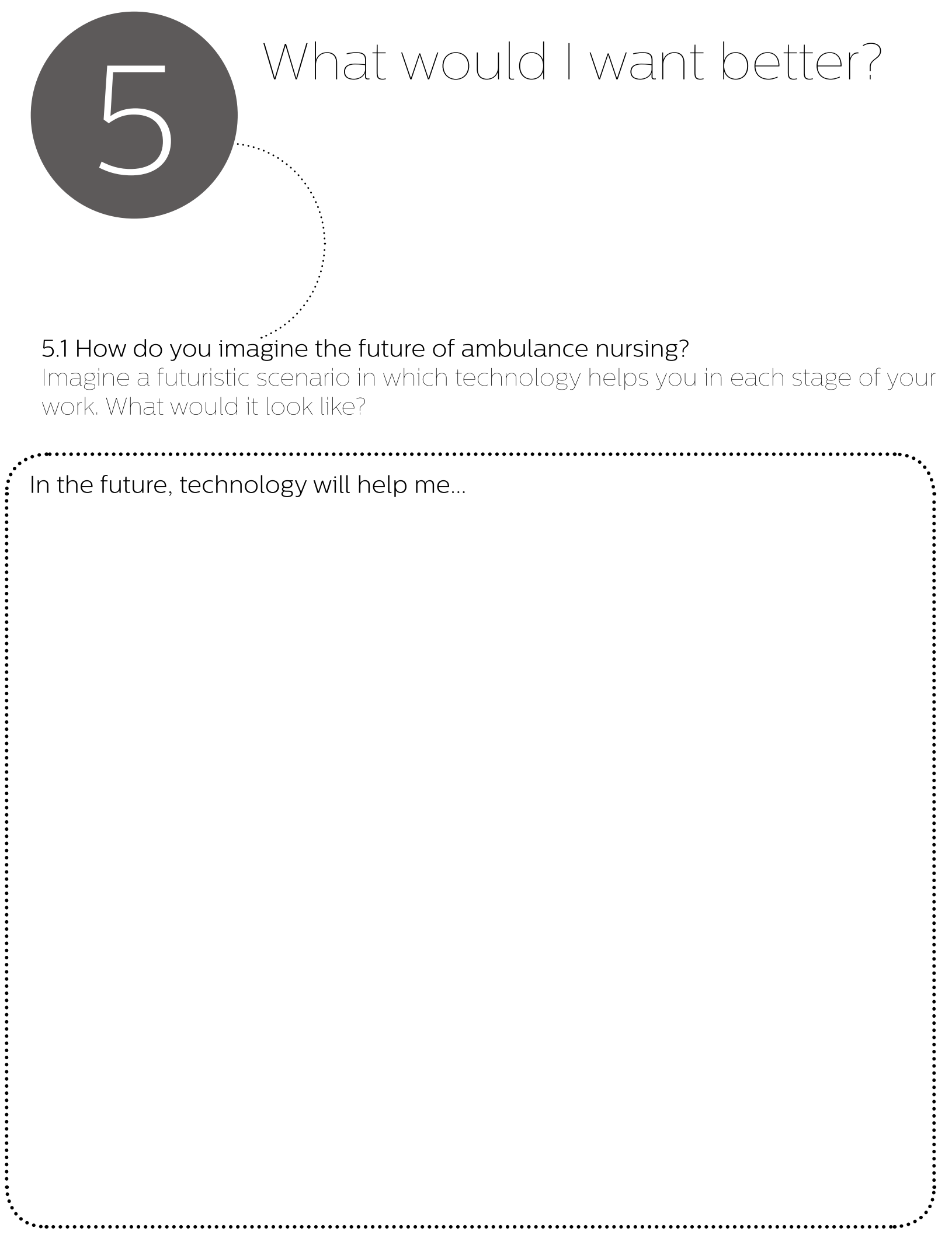

Bonus question: If you could have a superpower helping performing your job more comfortably and efficiently, what would it be?

you could get every capability you desire. What would you ask for? Answer in

the box, then give an example of a situation in which this could help you.

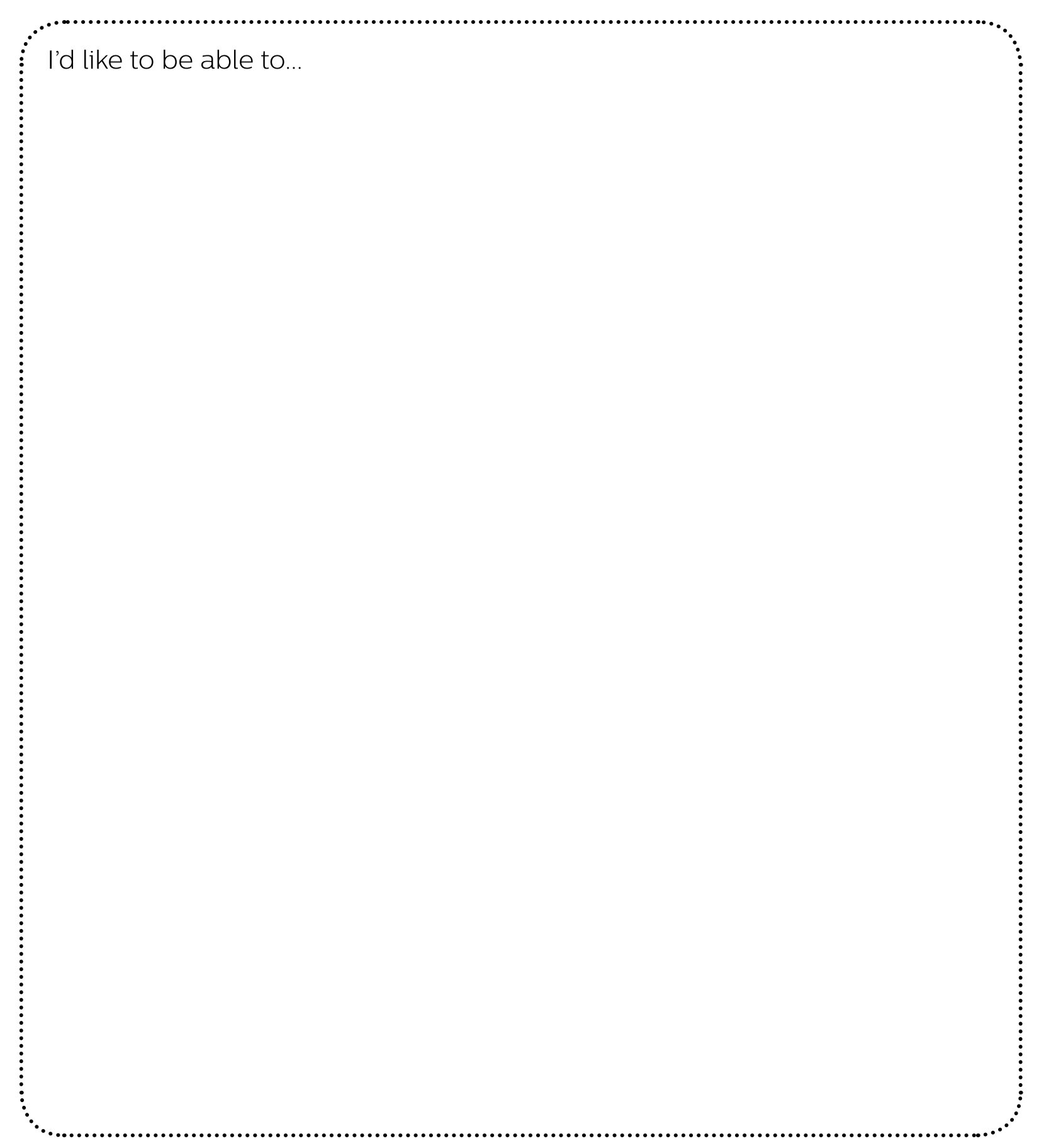



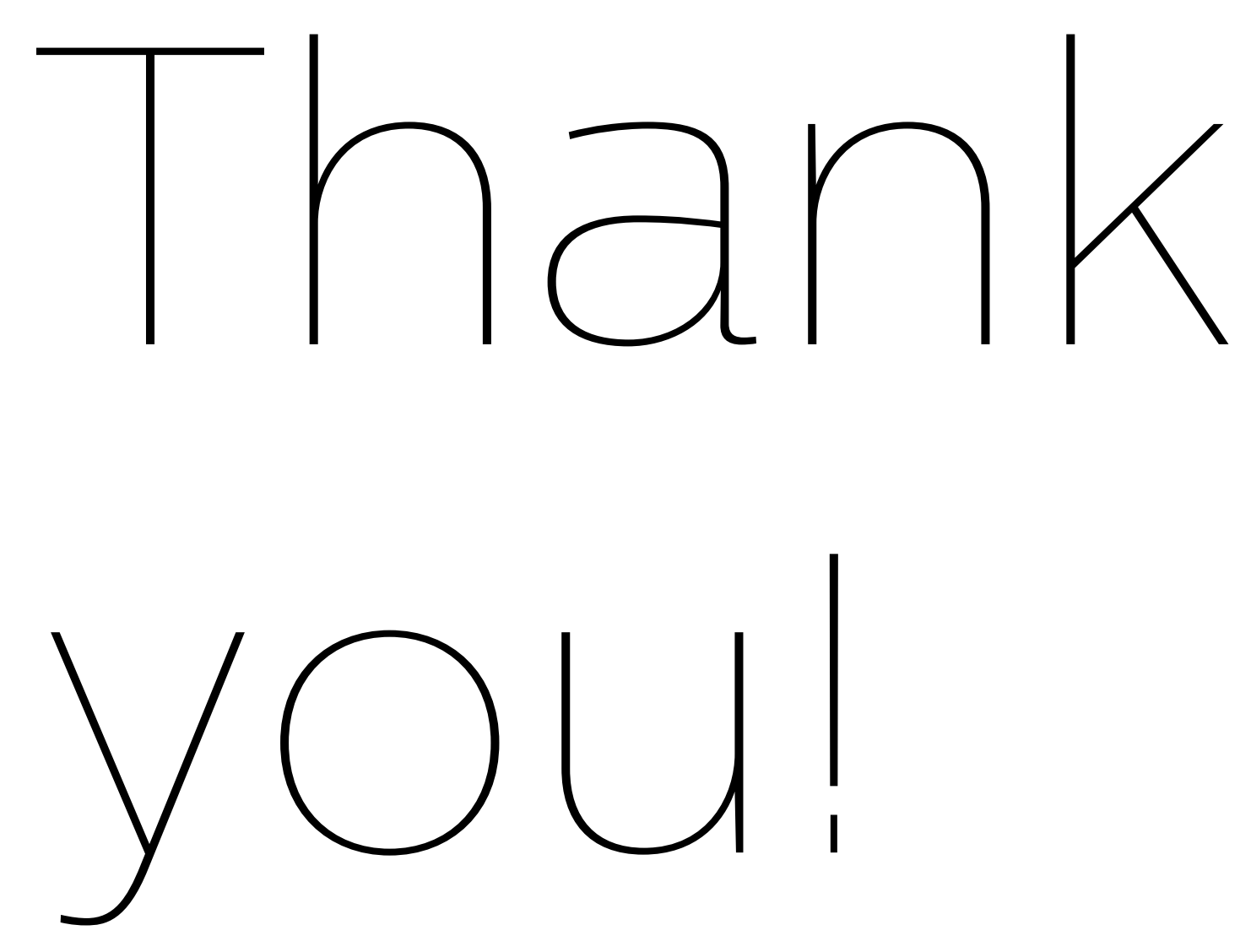

This was the last exercise. To conclude, sketch yourself on the cover where the white face is. Now your own personal guide to ambulance nursing is complete.
The booklet is over.

You can use the following pages for every additional remark, wish, description.

experience, story you would like to share. 


\section{Appendix V}

Highlights from the expert interviews.

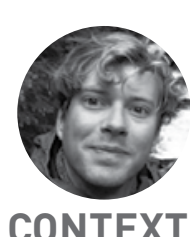

CONTEXT FACTORS

The delivery of patient information to the response team in the hospital
is never direct, but mediated by the is never direct, but mediated by the
blue line, who connects and organizes everything (like a 'spider in the net').

Emergency rooms and ambulance centers seem to be quite different of the work flow and emotional distress.

\section{PROBLEMS}

Currently, it seems that Ambulances an Emergency Rooms IT systems are no Some infrastructure that was mean to enable this are not used in practice. Direct, personal communication generally preferred.

\section{OPPORTUNITIES}

Sometimes,the response team gathering in the emergency room could the patient than what is provided by the blue line. To overcome this, medical staff often finds do-it-yourself solutions (e.g. smart-phone pictures from the accident shown to the emergency physician when arriving.

Eater broader assessments of the constitute a value for the Emergency Department (there's a project runnin or boarding ultrasound machines on board).

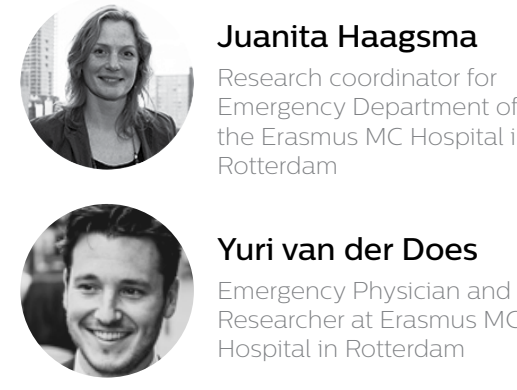

\section{CONTEXT FACTORS}

Teamwork: in hospitals in which Emergency Physicians are present in coordinating and managing the team which is being formed in response to every emergency.

\section{PROBLEMS}

The overfiling of the Emergency The reason doesn't lie in an excessive number of emergencies per hospital or in an inefficient reception system in the Emergency Department, as much as in the difficulty of freeing up beds by transferring stabilized patients to definitive care. This difficulty could arise from the difficulty to get ambulances to transport the patients home or to could not allow for non- The MKA transportations). Also, the transfer of patients to other Departments of a same hospital is often a lengthy and difficult process

This difficulty to output patients could sometimes bring to the impossibility of the Emergency Department to accept
'False' or 'non-updated' patien in Emergency Departments Every assessment system has to allow certain degree of underestimation and overestimation: in Emergency Care, a bit of overestimation tends to be used in order to avoid dangerous outcomes Still, the 'colour coded' assessment only time, and doesn't provide inf waiting about how the case is evolving vey urgent cases (black or red) could quickly become green, as soon as a first aid is given (example: patient choking on chewing gum is a black, but could get a green as soon as the gum is removed Continuous re-assessments of patients conditions are preferable to a singula

\section{OPPORTUNITIES}

Receiving extensive data about the patients could not constitute a absolute advantage in the Emergency Ward : the staff wants to know the to read everssues (heres not time Emergency Denart medical history. and experienced in staris traned

Automatic vital signs analysisdecisional support system: the problem with this is that, especially in Emergency Care, only relying on numbers can be very dangerous. Machines can be inaccurate, or people taking the unreliable (es because momentanty good Emergency Physician is usually critical about the reliability of every important piece of data concerning the patient he's treating.
Live protocol reminders don't constitute Emergency department as proto should be remembered receiving an emergency. There's generally no time to express doubts and have them solved. All-time reminders as the ones already present in from of posters around the Ward, fulfill the function of refreshing the staff memory A system that manages the access to patient informations in a coherent form across all his emergency journey would represent a disruptive improvemen in Emergency Care. Privacy would still constitute a great challenge to overcome, but some solutions could be possible (e.g. a safe account in which ambulance workers can log-in and blind' mode).

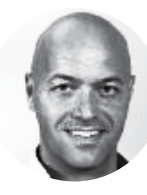

CONTEXT FACTORS

in under-urbanized or rural areas, the incidence of 'false' emergencies at the Emergency Department is almost negligible (about 95\% in Delft are concrete emergencies requiring the intervention of a doctor)

intual feedback and the Emergency Department In particular the Emergency Department staff gives feedback about the clarity, completeness and time efficiency of the patient presentation.

In Delft, the overcrowding of the Emergency Department is very rarely a problem. The Emergency Physicians and operating clams both icu beds sometimes causes delays in planned operations, but emergencies must have the priority. The internal hospital policy is made in a way to encourage departments to accept patients from the Emergency Department. Thanks to these measures and perhaps on almost never the need of losing the Department (which is seen as a very measure which requires the signed approval of the hospital board.)
Receiving extensive information about he patient before his or her arrival from the point of view of Emergency Physicians. In fact, there could be no time to prepare for upcoming patients Starting the case assessment by receiving a face-to-face description of many advantages: among others, the possibility to make questions.

\section{PROBLEMS}

The application of the color coded system, used to prioritize the Emergency Deparment queue, is often suboptima minutes, but a more accurate triage $(10$ to 15 minutes long) is usually performed Also, 'urgent looking' patient are often and go directly to triage. All in all, color coding is now often just another time

\section{OPPORTUNITIES}

Having patient's ID documents photographs sent to the Emergency patients could speed up administrative procedures of around 5-10 minutes per case

Ambulance nurses could remember more often to bring patientsmedications would solve the diffirt emergency physicions foce wh patients don't remember the exact name of their medicines. 


\section{Appendix VI}

Flow and ambulance nursing: observations

FLOW AND PERSONAL INVOLVEMENT

Some additional consideration can be drafted
out of the comparison between classic flow theory and the observable effects of high levels of focus of ambulance nurses at work

In fact, while points like Csikszentminaly's have been directly observed or self-reported in EMS, a fundamental dissimilarity has been outlined. This difference regards the fact that while in orthodox flow theory the 'ecstatic state is associated with depersonalization and isolation from social stimuli, in ambulance nursing this tendency seems to

An important part of the nursing profession is to stay open, reassuring and warm towards the small scale of the hereby presented research, somehow kept into consideration by the nurses reaching a state of flow, who tend to be even more caring than usual when particularly busy

\section{THE HYPOTHESIS}

A possible implication of this observation for the general flow theory is that, more than pushing towards de-personalization,
the state of flow can be triggered by repersonalization, as if a mind's strategy for reaching superior states of concentration and overcoming personal distractions when extremely necessary would be to 'turn' into

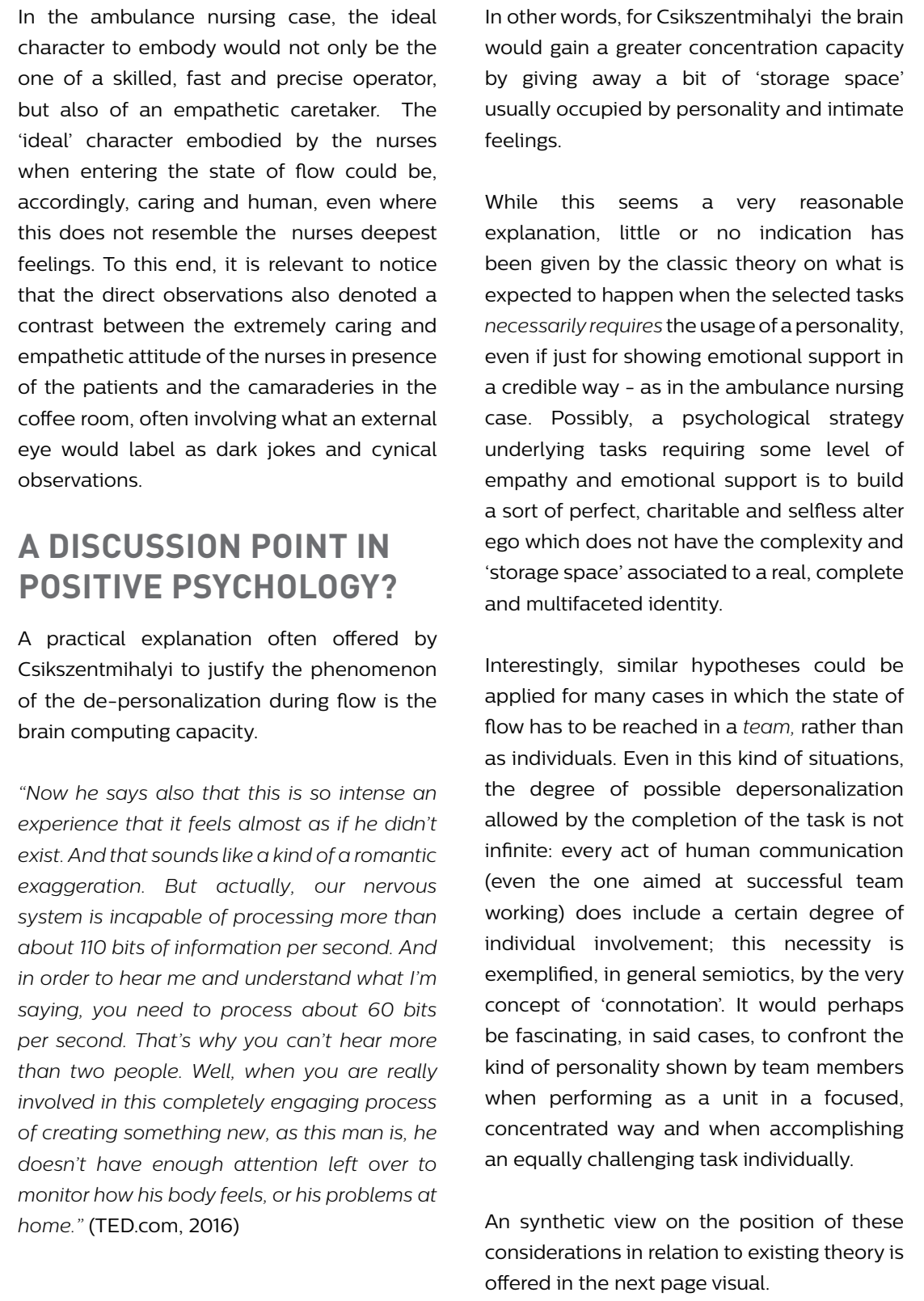

Interestingly, similar hypotheses could be applied for many cases in which the state of now has to be reached in a team, rather than as individuals. Even in this kind of situations, allowed by the complete depersonalization infinite: every act of human con lask is not (even the one aimed at successul team working) does include a certain degree of individual involvement; this necessity is exemplified, in general semiotics, by the very concept of 'connotation'. It would perhaps be fascinating, in said cases, to confront the hin of personality shown by team members when performing as a unit in a focused, an equally challenging task individually.

An synthetic view on the position of these considerations in relation to existing theory is offered in the next page visual.

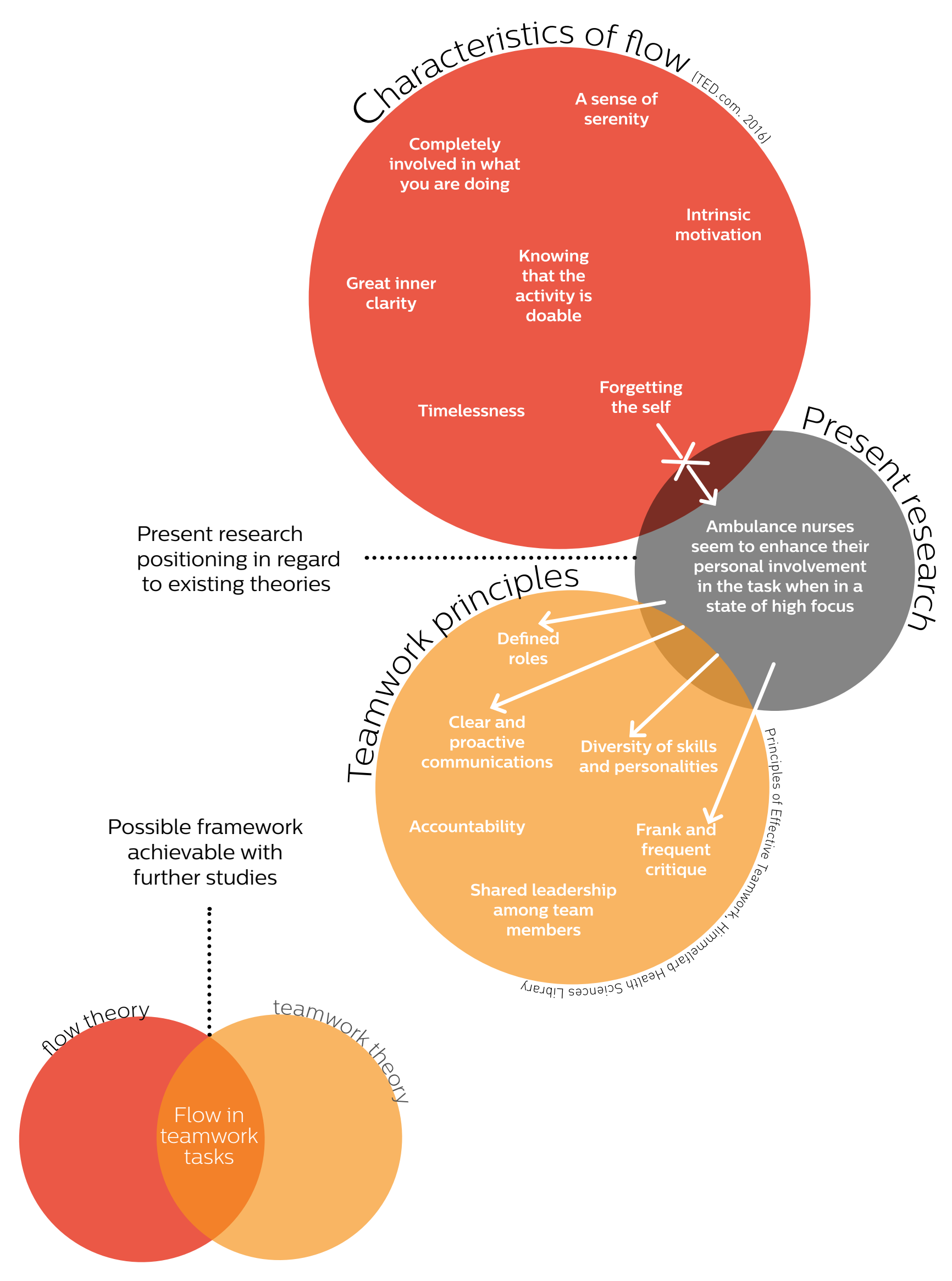




\section{Appendix VII}

List of problems reported from the user research

DIFFICULTY TO FOLLOW/ REMEMBER THE

PROTOCOL

The existence of difficulties remembering or following the protoco cexplich askd to park the prits in the recognized it as a real problem, althous not particularly frequent nor severe (one of them declared to "follow common Sense' in every case of protocol doubts. An example of difficulties in followin the protocol is the obligation to wear safety belts in every situation of transport obligation that nurses often disregard case the treatments they are provid

PHYSICAL DISTRESS

The problem of the physical strain related the ambulance nursing job was pointed tor the stretcher and in general the action .

COORDINATION / COMMUNICATION PROBLEMS

The opportunity to investigate this problem area was as suggested in the 'distributed team' cognitive framework illustra
the preliminary research chapter

Therefore, the existence of problems communicating and coordinating with the pest of the team was explichy ashed
Apart from this, the direct observation secifically noise disturbance in the use of he transceiver with the control room an the hospital. conversely, include mostly cases of misunderstanding or conflicting interests with the communication address

Examples a

- Difficulty of the ambulance nurses to categorize the case they are (eg. respiratory psychiatric) when alerting the destination hospital. This constituted a problem as the dispatch nurse in the hospital has the need to gain the needed specialties professionals for the case (especially in hospitalls who don't contemplate emergency physic a stable professional figure).

from the control room operator

when departing for a call. One to ask more on the destination emergency, apart from the basic severity and position, were usually left unaccomplished by the control room operator, who has to keep free for receing calls and usually tries talking to the nurses.

to contact General Practitioners knowing the patient medical history also due to their limited availability only office hours). fhe ambulance shift pointed out the

The observed coordination problems

to receive exe ambulance nurses

\section{EMOTIVE DISTRESS}

An exceptionally efficient and pragmatic approach to emotively charged situation was observed in ambulance nursing. By mean of experience, of the 'cold blood

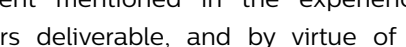
sots defiverable, and by virtue of colleagues about injuries and diseases the nurses seem to possess an abovethe-average capacity to defend their al resilience.

Still, some experiences are still capable of shaking nurses stability, especially in the case of pediatric cases or unexperienced crew members. Episodes of self-mistrust. shock, difficulty to go back to work, desire to quit, and depression, were mentioned as consequences of emotionally heavy NON-COMPLIANT PATIENTS

Especially from the individual interviews with Dutch participants, it emerged as problem the difficulty of gaining the full collaboration of the rescued patient

The tendent

to to the patient care to the nurses. mainly for motivations of legality (e.g. use of drugs, of alcohol while driving)

The tendency if some patient no to admit stable conditions such as dependence from alcohol, such as depression usually for personal reasons.
The unwillingness of some patient unwillingness which pus in the very difficult position of havin medical care.

The difficulty of gaining reliable mentally ill patients, especially the case of psychiatric conditions causing forgetfulness (dementia alzheimer disease)

PERSONAL SAFETY THREATS

A small number of participants of the Andividual interviews and a relevant websites mentioned the existence a personal safety risk related to the profession of ambulance nursing. Examples of the risks are:

Being involved in violent or unsafe situations (e.g. street fights, fires) while giving assistance. Being contaminated by infectious diseases by the patients traveling on the ambulance at high

LONG/DIFFICULT

PAPERWORK

Almost the totality of involved participants mentioned the time consumption and difficulty of filing up the administrative report to deliver to the ambulance

Regarding the difficulty, about two current report format to describe all of the spectrum of emergencies, being too focused on car accidents or trauma cases in general.

DIFFICULTY TO GET INFO Almost the totality of the involved problematic to gather the information necessary to proceed with an operative rescue plan, especially in case nresponsive patients amples of missing information include Paterts medce especially latest tests results, existence of chronic conditions or alleries

Medication intake, for which the " proofs of medications

Data relevant to the anamness which cannot be gained by the nurses with the existing equipment, in particular diagnostic imaging (X-rays, ultrasounds), quick blood tests, more accurate screenings of respiratory, Cardiac and Erculotory

DIFFICULTY TO RECALL NFO

Almost the totality of involved participants informations from the case as a common problem in ambulance nursing. A relevan number of them reported to make use of side solutions such as writing quick notes on their gloves or asking the aid of the This problem can be perhaps better understood by considering the high level of detail requested to nurses in writin the administrative report, including exact quantities of each medication administrated. Also, cases in which

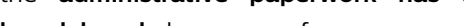
be delayed because of more urgent ectedly worsen this

QUIPMENT FAILURES

A relevant number of participants maffunctioning or failing as a not very frequent but extremely severe accident ambulance nursing. Collected complain The ECG monitor

The though-book (running Windows $\mathrm{XP}$ as an operative system in some The communication especially the transceivers.
E.R. OVERCROWDING number of participants mentioned the case of finding problems in handing over due to the ward overcrowding. This problem was also largely testifed by Emergency Physicians consulted durin the interviews with experts (see Append $\checkmark$ for more details). 


\section{Appendix VIII}

Creative session schedule

\section{Effortless interactions for Emergency Care}

\section{Session schedule}

11.00 Choose a superhero and tag yourself

11.05 Memerize

11.25 Problem presentation: Workflow, characters needs, problems

11.35 $\mathrm{H} 2 \mathrm{~s} 1,2,3$

\subsection{Doom scenario}

12.03 H2s 4, 5

12.16 Clustering

When finished clustering and then only: I unch

12.25 Voting ( 3 for like, 3 for coolest interaction and 3

for ambulance nurse friendliness)

12.35 Choosing

12.36 Elaborating concept

13.00 Preparing presentation

13.20 Presentations 


\title{
Appendix IX
}

\author{
List of requirements
}

\section{PERFORMANCE \\ The device permits to capture and \\ The device sho \\ hands and eyes-free. \\ The device transmits what captured \\ (in a text format) to a connected \\ radio calls of selected devices. \\ The device should provide different \\ feedbacks, including a) ongoing calls \\ voice input. \\ The device should provide an \\ ENVIRONMENT \\ The device should be suitable for \\ indoor and outdoor use. \\ circumstances up and until 5 ATM. \\ The device should operate at \\ emperatures between -10 and 40 \\ The device can be stored at \\ temperatures between -20 and 60}

\section{LIFE IN SERVICE}

The device should be shock resistant
The device should be able to withstand a force of $200 \mathrm{~N}$

\section{MAINTENANCE}

Device is $c$

The device should be able to be

cleaned with water and soap

\section{QUANTITY}

Mainly Dutch, UK, USA and

PRODUCTION FACILITIES

Use facilities connected to Philips

SIZE \& WEIGHT

Device should be wearable with little or no long-term discomfort.

AESTHETIC,

APPEARANCE \& FINISH The device appearance should be discrete and clear

\section{MATERIALS}

All materials in conformity with EN 1888 and Toxicity (EN71-3) corrosion are allowed

\section{PRODUCT LIFE SPAN}

Expected life cycle of 3 years intensive use

STANDARDS, RULES \& LEGISLATIONS

The device should avoid arousing privacy issues

ads to achere to

safety regulations

safety regulations

\section{ERGONOMICS}

Device should fit adults (18-60) Device allows for diverse activities

(walking, running)

RELIABILITY

Data should be backed up (cloud) The device should be able to

\section{SAFETY}

Should not lead to skin damage

- Should not use harmful materials

chould no contain elements that

Should not emit

.

cause harm to the continued user.

PRODUCT POLICY

The device should fit the current Philips portfolio

SOCIETAL \& POLITICAL IMPLICATIONS

The device design should not infringe with registered patents. If relevant, the device should be protected by a registered patent

INSTALLATION \&

INITIATION OF USE

Easy to install and remove without

\section{WISHES}

$$
\begin{aligned}
& \text { Cost-efficacy } \\
& \text { Most seamless integration with } \\
& \text { Health suite } \\
& \text { Integrate third party data sources } \\
& \text { Create peer to peer connection } \\
& \text { within nurses } \\
& \text { Product reduces perceived } \\
& \text { complexity of ambulance nursing } \\
& \text { Enhance nurse--patient connection } \\
& \text { Appearance least association with } \\
& \text { asthma } \\
& \text { As light as possible } \\
& \text { As compact as possible } \\
& \text { The nurse should not be feel } \\
& \text { controlled by the device } \\
& \text { The device may not cause distraction } \\
& \text { in use } \\
& \text { Over time the language recognition } \\
& \text { function should be able to evolve } \\
& \text { and get customized to the user. } \\
& \text { The nurses should see the inherent } \\
& \text { beneifts of using the device } \\
& \text { The system should allow real time } \\
& \text { monitoring of nurses actions by E.R. } \\
& \text { Personnel. }
\end{aligned}
$$




\section{Appendix X}

Weighted criteria choice

Feasibility

Viability

Desirability

\begin{tabular}{|lcccc}
\hline Criteria & Achievable/Realistic & Fit with Healthsuite(data) & Time saving & \\
Achievable/Realistic & & 1 & 0 \\
Fit with Healthsuite(data) & 0 & 0 & 1 \\
Time saving & 1 & 0 & 0 \\
Low Risk & 1 & 0 & 1 \\
Innovativeness & 1 & 0 & 1 \\
Patient Value & 1 & 0 & 1 \\
Brand Fit & 1 & 1 & 1 \\
Likeable & 1 & 0 & 1 \\
Effortless Interaction & 0 & 0 & 1 \\
Low Threshold for Users & 0 & & \\
\hline
\end{tabular}

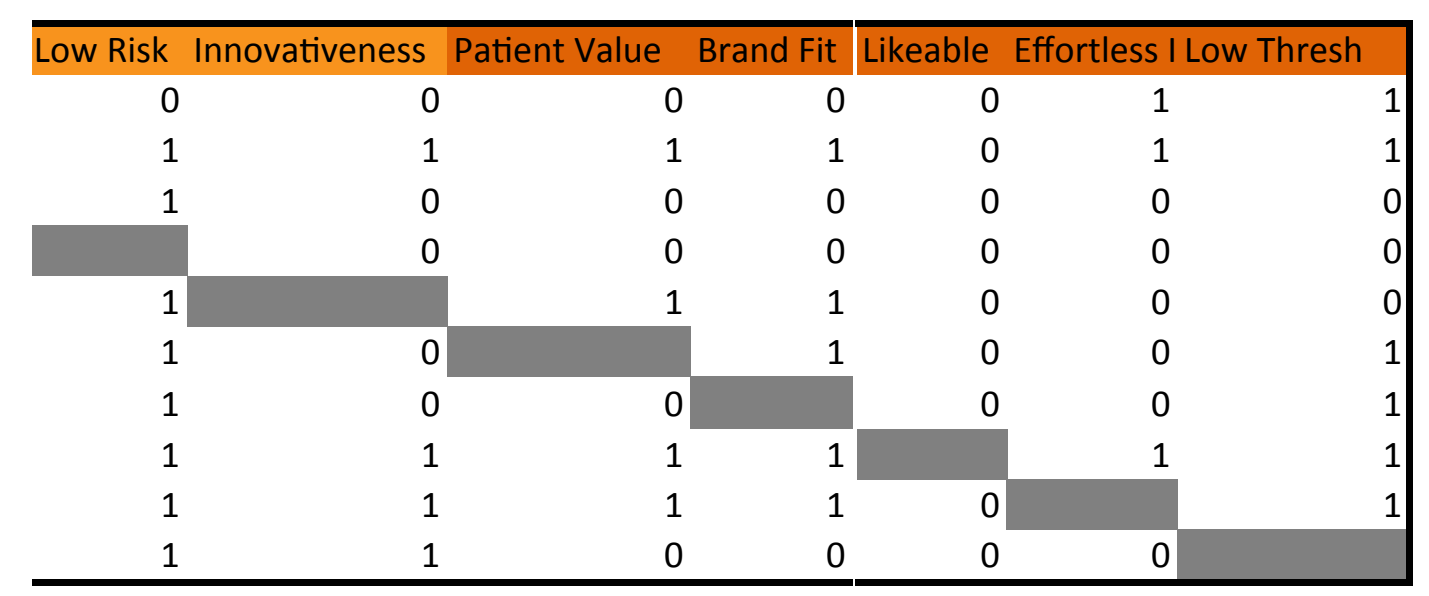

\begin{tabular}{|lrl}
\hline \multicolumn{2}{l}{ weight } & \\
Achievable/Realistic & 0.066666667 & 2 \\
Fit with Healthsuite(data) & 0.155555556 & 2 \\
Time Saving & 0.044444444 & 4 \\
Low Risk & 0.022222222 & 4 \\
Innovativeness & 0.111111111 & 2 \\
Patient Value & 0.111111111 & 1 \\
Brand Fit & 0.088888889 & 1 \\
Likeable & 0.2 & 3 \\
Effortless Interaction & 0.133333333 & 1 \\
Low Threshold for Users & 0.066666667 & 4 \\
& & 24 \\
\hline
\end{tabular}

\begin{tabular}{|c|c|c|c|c|}
\hline \multirow[b]{2}{*}{ Direction 2} & \multirow[b]{2}{*}{ Direction 3} & \multicolumn{2}{|c|}{ total weighted score } & \multirow[b]{2}{*}{ Direction 3} \\
\hline & & Direction 1 & Direction 2 & \\
\hline 3 & 1 & 0.13333 & 0.2 & 0.066667 \\
\hline 1 & 3 & 0.31111 & 0.155555556 & 0.466667 \\
\hline 3 & 1 & 0.17778 & 0.133333333 & 0.044444 \\
\hline 3 & 1 & 0.08889 & 0.066666667 & 0.022222 \\
\hline 3 & 4 & 0.22222 & 0.333333333 & 0.444444 \\
\hline 3 & 4 & 0.11111 & 0.333333333 & 0.444444 \\
\hline 4 & 3 & 0.08889 & 0.355555556 & 0.266667 \\
\hline 3 & 1 & 0.6 & 0.6 & 0.2 \\
\hline 4 & 2 & 0.13333 & 0.533333333 & 0.266667 \\
\hline 3 & 1 & 0.26667 & 0.2 & 0.066667 \\
\hline 30 & 21 & 2.13333 & 2.911111111 & 2.288889 \\
\hline
\end{tabular}




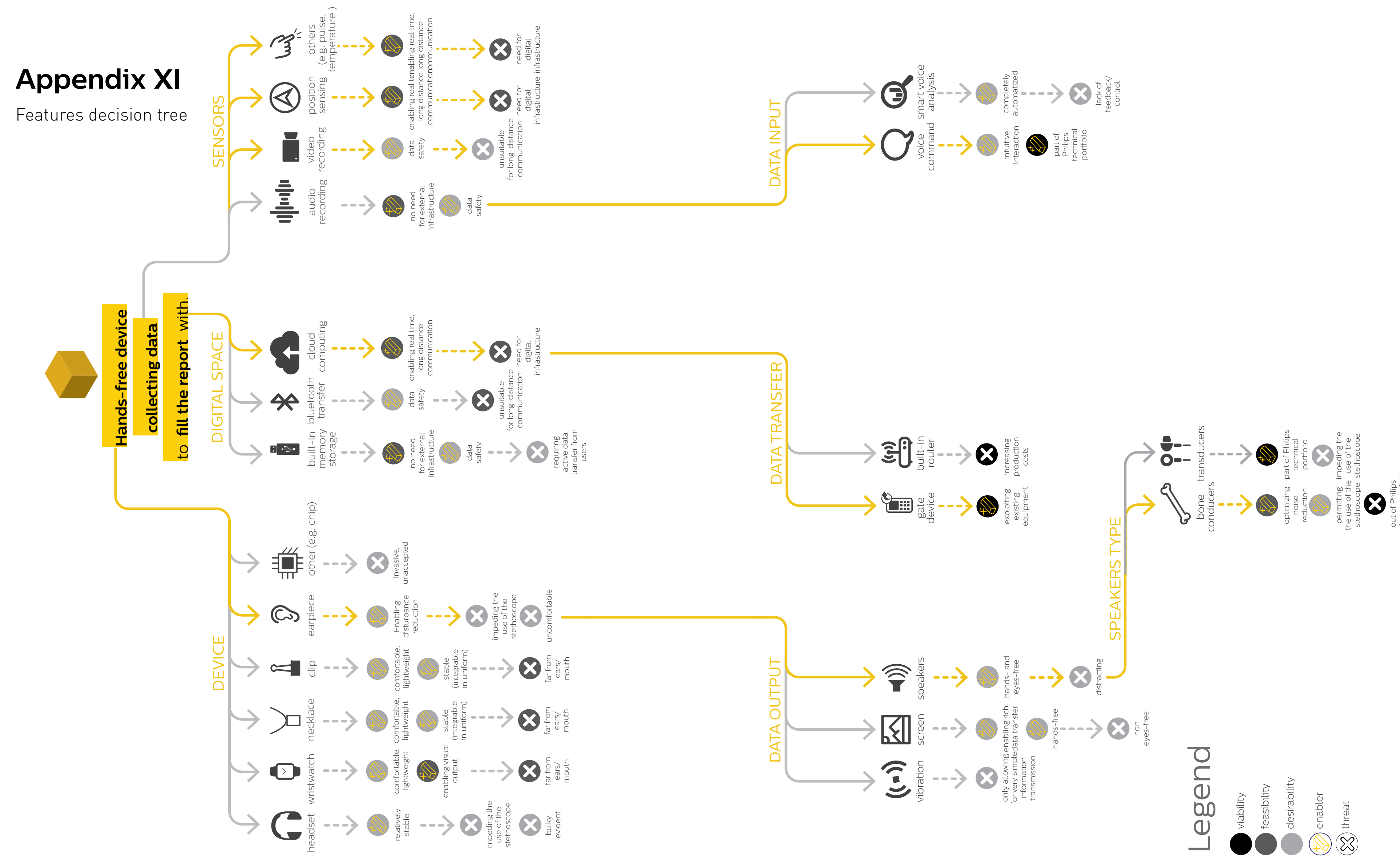




\section{Appendix XII}

Test protocols

Introduction

Hello and welcome to the test of livesync, a product meant to facilitate the and the later reporting

Before I describe you the details of the test I want to thank you for your participation and stress that, as a voluntary, you participation at any time.

During the test, I will take pictures and ecord of you, but not identify you by name in any further reports. Furthe uses of records and data will be subject standard data use policies which protect the anonymity of mindivduals and

The designed product is an earpiece to be This earpiece is capable of capturin information relevant to the reporting once you speak them out by voice. For instance you say 'pulse, 50', this piece of data will activate the earplece to put ' 50 ' in the feld reserved for "pulse in the report. So whenever you will start to fill the report you will ynd hat mirmation already

When something relevant to the report is captured by the earpiece, this will provide activates $\mathrm{mp} 3$ record of feedback soun from the smart phone

Also, the earpiece can be used to receive and make phoce calls wr hout using your
Whenever you will want to start checking the rescue report, you will find it already Aled in with informations. At that completeness of this report and add what you think is missing.

fter the test, I will proceed with a short questionnaire in which we will have the chance to discuss how did it go with the simulation of the product usage.

Test procedure explanatio As mentioned, this will be a simulation of

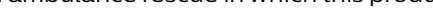
Wil be used. One of my team members (introduces) whl act as a patient and how what is the case he was in reveal o simulate to obe nin a more realistc scenario. However. I can anticipate to you that is a very common and simple case so no knowledge of rare protocols requested.

beg you to behave as you would do pormally on the scene of an emergency you Vou decide that something should be the piece of information will have been registered.

For what concerns the possible treatme to provide, I will ask you not to proceed in any invasive one: for instance, in case you would proceed firh an IV placement patient's skin. Still, if what you are doin is something you think should be in the report, speak it out loud and wait for the confirmation sound. if you will hear the confrmation sount by protocol, please just act it out,
When you decide so, you can stop patient (as if he was

Then, I will give you the pre-filled report you are used to fill in this ane that center, but the fields you will find are analogous. Please review this report and complete what you think is missing If some informations are different from what you said duning the simulation, don missing data. When you are satisfied with

After the test, you can tell me how it went and 'll make you some short questions Any questions?

-Do you want to go for a small try-out before starting the simulation?

(Checking information speaking and

(After tryout) Are you ready to start? bother correcting them: only focus on
After simulation questionnaire

Effortless interactions for Emergency Care

Test

Questionnaire

\section{Open questions}

How did it go?

How was the feeling of receiving the feedback sound?

(Options card 1, 2, 3, 4)

What do you think of the functionalities of this system? (speech recognition, hands-free calling,
decisional aid)

Which problems do you see as related to the use of such a system in common practice?

Do you have any suggestion about how to improve this system?

Which one of the chosen earpiece form would you prefer to wear on duties?

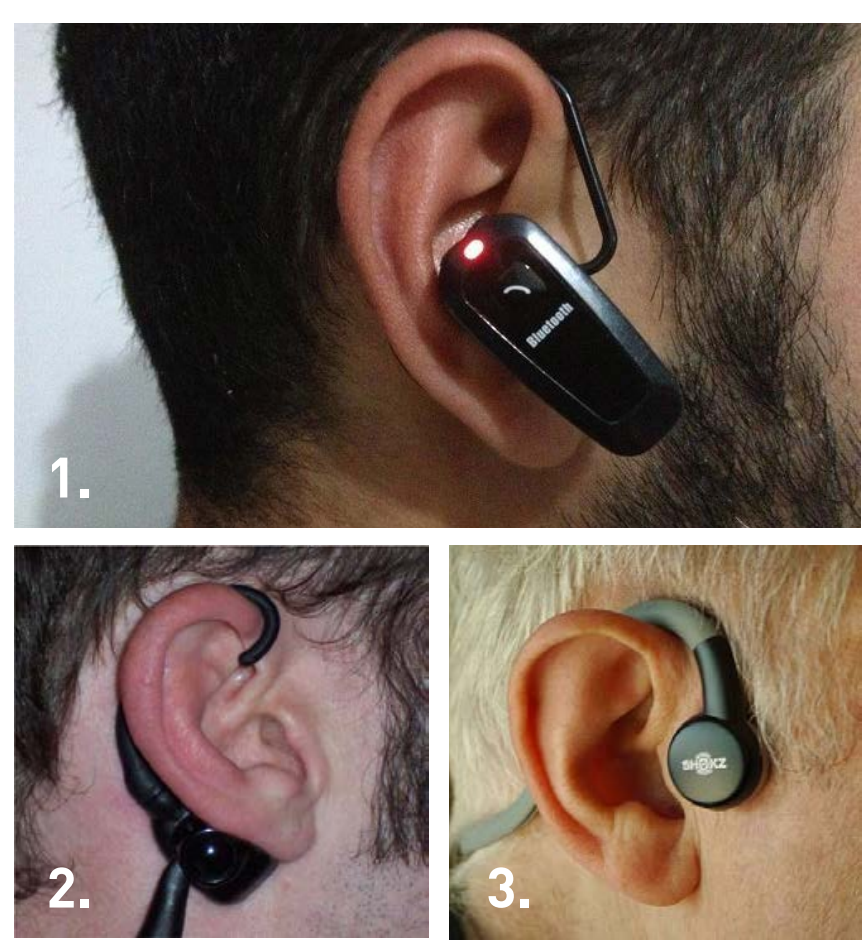

More remarks? 
Options cards 1,2,3.4 Do you think this system would have impact on saving your time at work? Please choose the option
closer to your opinion, then explain me the motivations of your choice.

$\square$ ' think it would make me waste time

$\square$ I don't think this would have any impact on the time I spend doing things.

$\square$ I think this would save me time

While simulating the rescue, did you notice any change in the effort you put in reporting? Please choose
the option closer to your opinion, then explain me the motivations of your choice.
While simulating the rescue, did you notice any change in the attention you could reserve to the patient?
Please choose the option closer to your opinion, then explain me the motivations of your choice.

$\square$ । felt like I could focus on the patient less than I usually do and give him less support.

$\square$ No, I didn't notice any difference regarding the focus or support I could reserve on the

$\underset{\text { him better. }}{\square \text { did have the impression that I could reserve more attention to the patient and support }}$

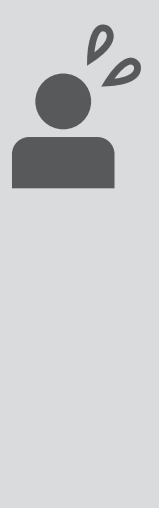

Survey structure summary

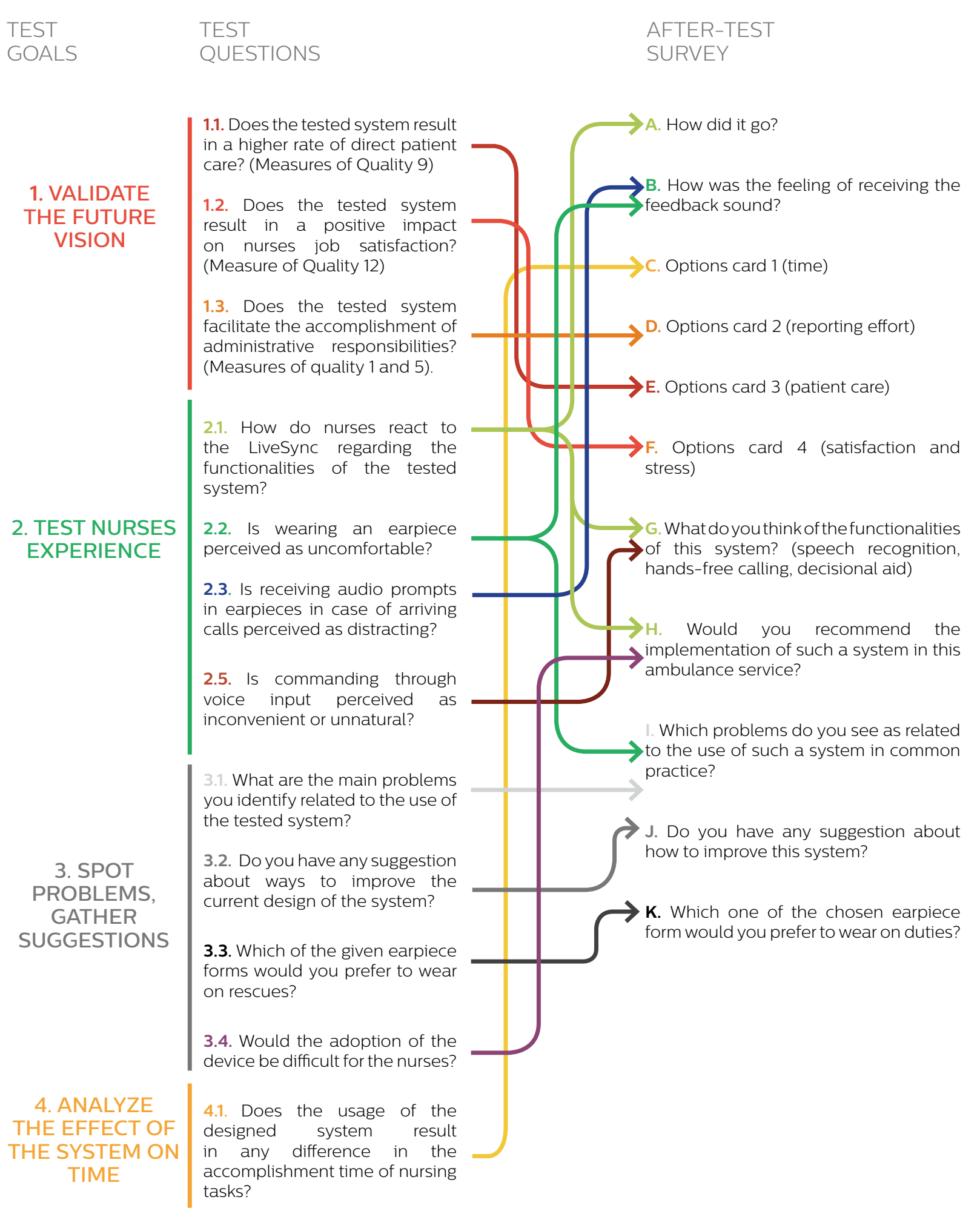




\section{Appendix XIII}

List of recognized keywords for report voice command

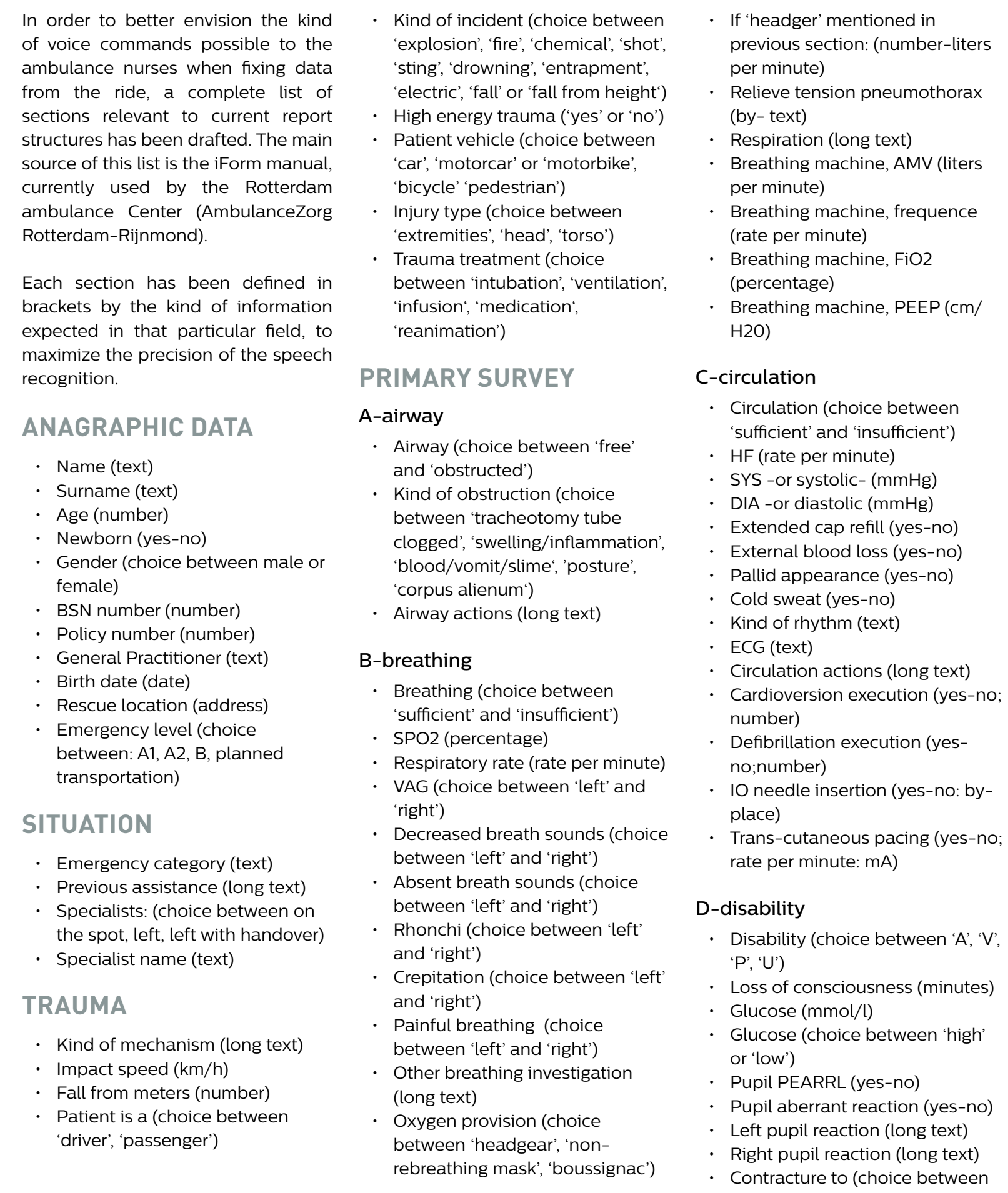

'left' and 'right')

- Nystagmus (choice between 'yes' or no) (choice between 'yes'

Comments on disability (long

text)

E-exposure

Hypothermia ('yes' or 'no')

. Hyperthermia ('yes' or 'no')

Comments on exposure (lons

text)

\section{MEASUREMENTS}

SPO2 (percentage)

- Respiratory rate (rate per minute) EtCo2 ( $\mathrm{mmHg}$ )

HF (rate per minute)

SYS -or systolic- $(\mathrm{mmHg})$

DIA -or diastolic ( $\mathrm{mmHg}$ )

Rythm (text)

Open eyes ('yes' or 'no')

- Locomotion (yes or no)

Verbal reaction ('yes' or 'no)
GCS (text)

RTS (text)

Glucose (mmol/l)

Glucose (choice between 'high'

or 'low')
Temperature (Celsius degrees)

Pain score (number)

Pupil PEARRL (yes-no)

Pupil aberrant reaction (yes-no)

. Left pupil reaction (long text)

Pediatric trauma score: length

(cm)

Weight (kg)

Open wound (yes-no)
Symptom (choice between 'stuffy', 'shock', 'haemorrhage' 'chestpain', 'collapse',
'acute abdomen pain',

'Loss of consciousness' or

unconsciousness', 'insult',

headache', 'fever', 'intoxication

hypothermia', 'hyperthermia', disease', 'mental ilness ')

A.M.P.L.E.

- Allergy (yes or no)

Allergy (text)

Medication intake (yes or no)

Medication intake (text)

Past history (yes or no)

Past history (text)

Last meal (time)

- Last meal (text)

MEDICATIONS

- Administered medication (name)

Administered medication

- Infused medication (name)

- Infused medication quantity

(number)

\section{WORKING DIAGNOSIS}

Diagnosis specialism (text)

Diagnosis specialism (case code)
Differential-secondary diagnosis

quantity (text)

Differential-secondary diagnosis

quantity (case code)

Working diagnosis explanation

(long text)

\section{END OF RIDE}

Attached files (text, pictures)
Type of transportation (choice between kind of transportation)

TIME LINE

Activate report (text) 


\section{Appendix XIV}

Screen shots from the landing page

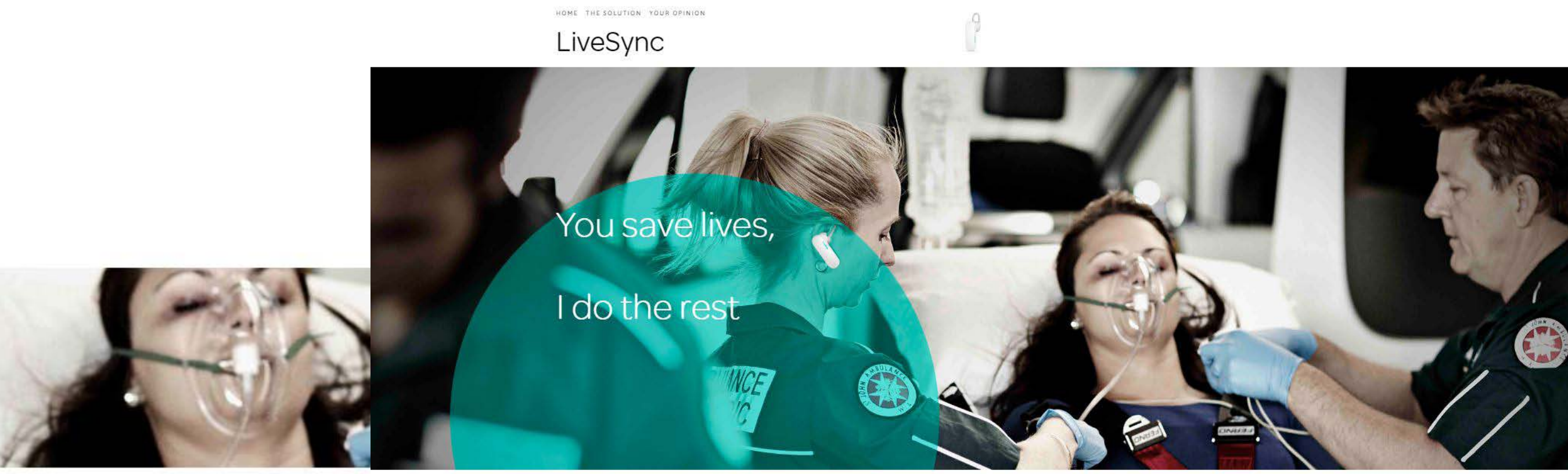

LiveSync

Welcome:

FASTER

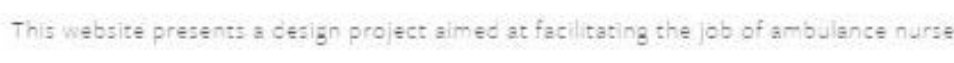

REPORTING

open to all the professionat if

Unlezs you want to stay updastec and choosa to give me your contact catsals, the ouestionnaire is anonymout

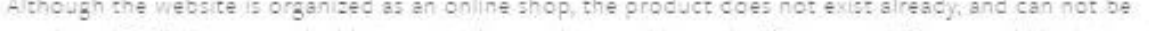

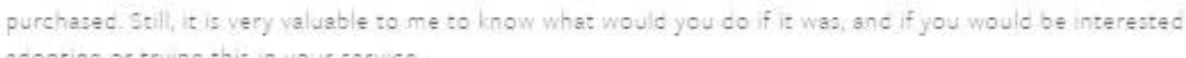

Plasse provide me w th your fasbback before Saturday the 27th of August.

Curious? Tell $m$ me what is your job and press start:

$\stackrel{P}{\longrightarrow} \longrightarrow 9 \longrightarrow$

Whas is your professi lonal role?
Ambulanse nurse

SUEMIT 


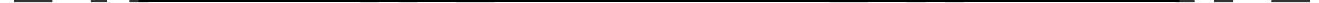

EASIER COMMUNICATION
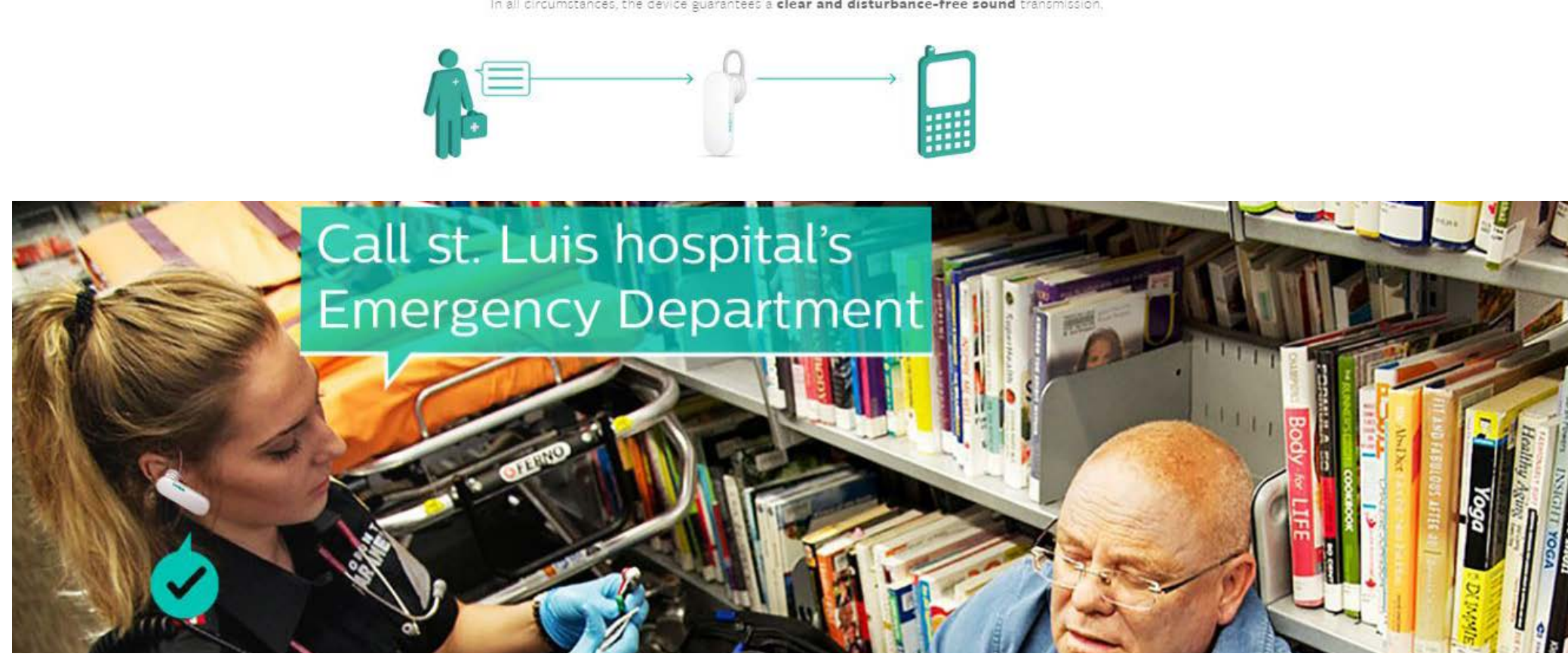

ACCESSIBLE INFORMATION<smiles>C1=CC2CC=CC2C1</smiles>

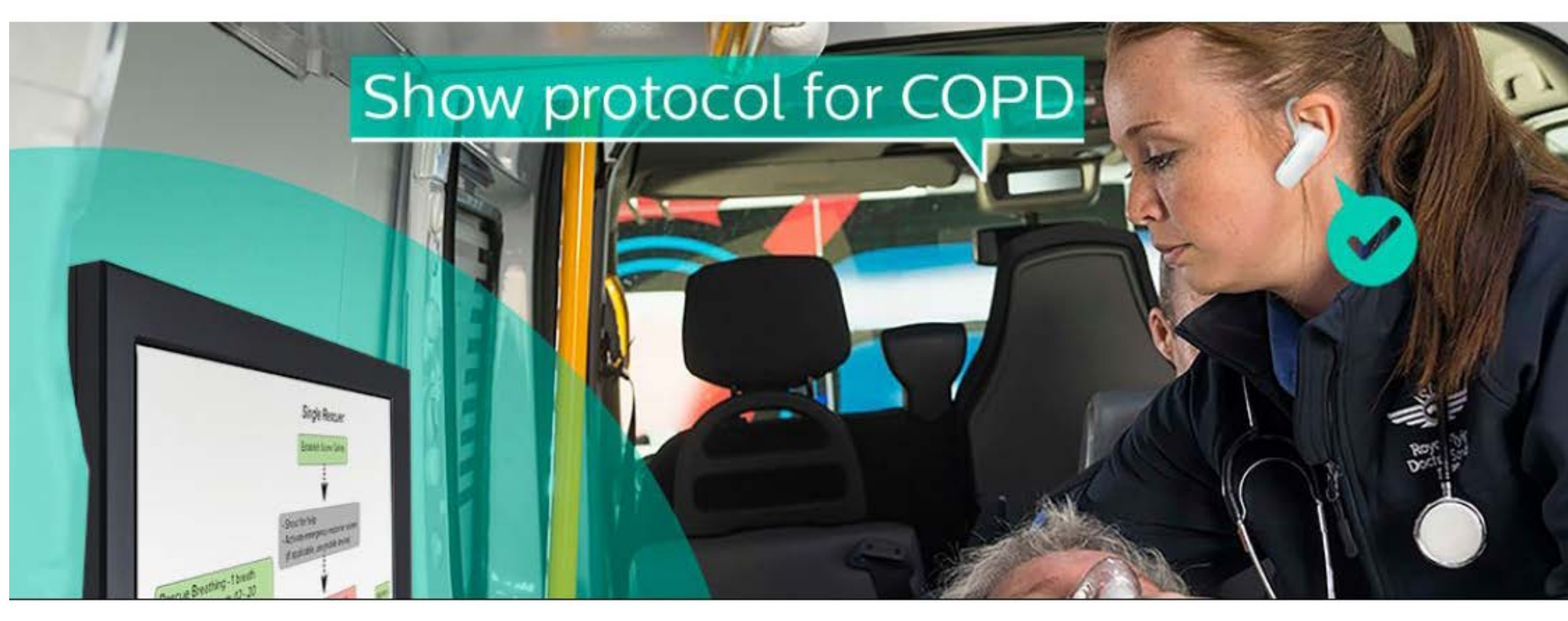




\section{What do you think?}

..all for 50 cents per case* Please give your feedback in this small form

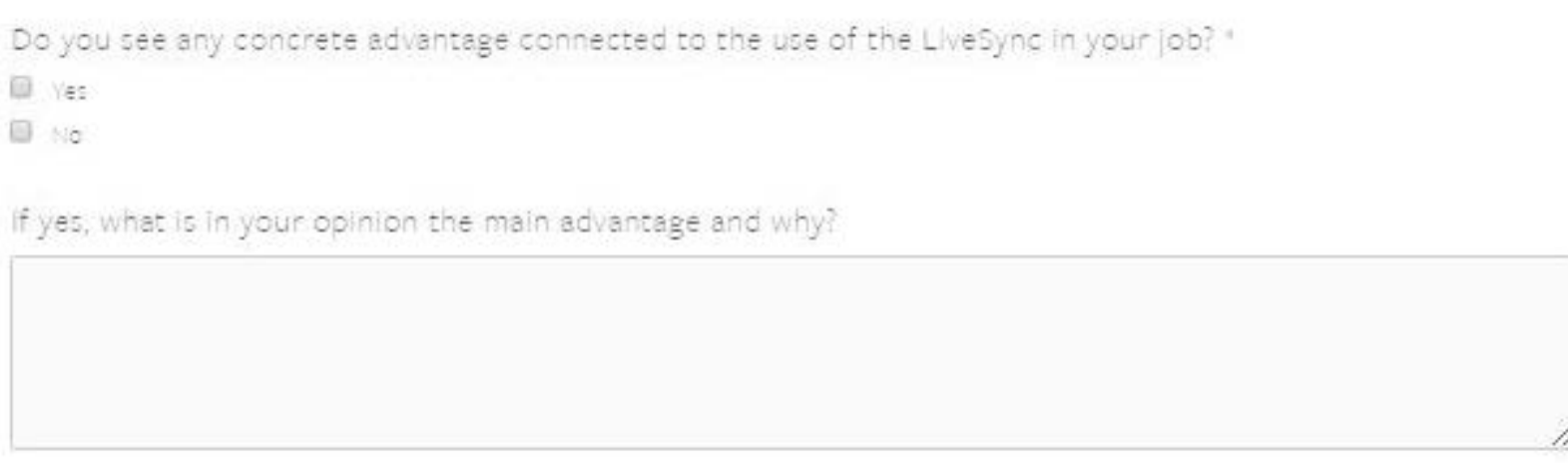

Do you see any problem connected to the usage of the Live sync in your job? Dis:

$3 \times$

If $s 0$, which one $(s)$ :

Would you suggest a free tryout of this system to your ambulance service?

$\square$

Why? 
HOME THE SOLUTION YOUR OPINION

\section{LiveSync}

Suggestions?

I would change the idea in this way.

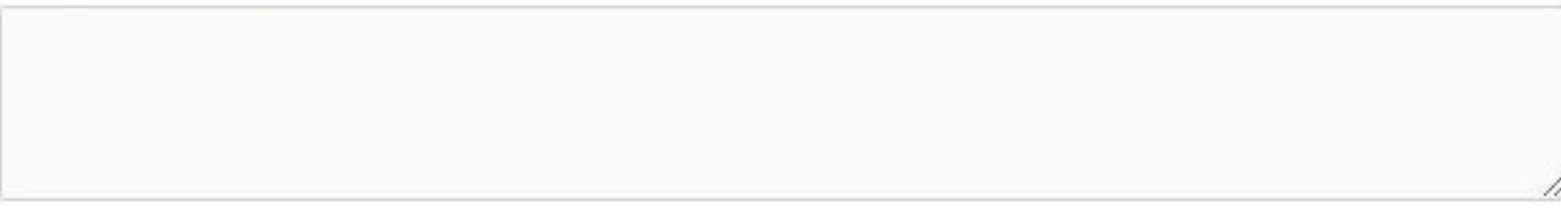

SUBMIT

Comments

Use this space for any other remark you would like to provide me

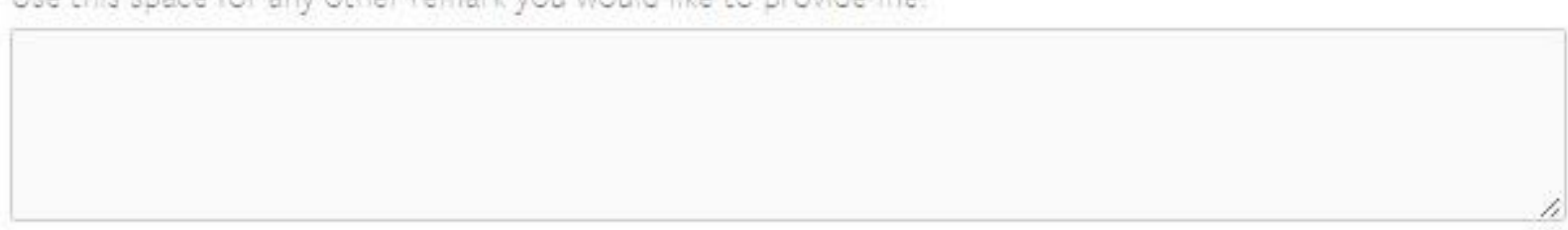

SUBMIT

\section{Thank you!}

If you wish to ctay updated about this project, subscribe to the news etter

SUBSCRIBE

Sign up with your email address to receive news and updates.

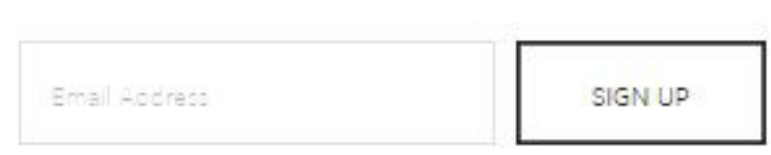

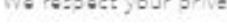

STOCKISTS FAO PRIVACY TERMS OF USE CONTACT

\section{Thank you!}




\section{Appendix XV}

Personal learning points (and hints for future graduating students).

\begin{tabular}{|c|c|c|}
\hline 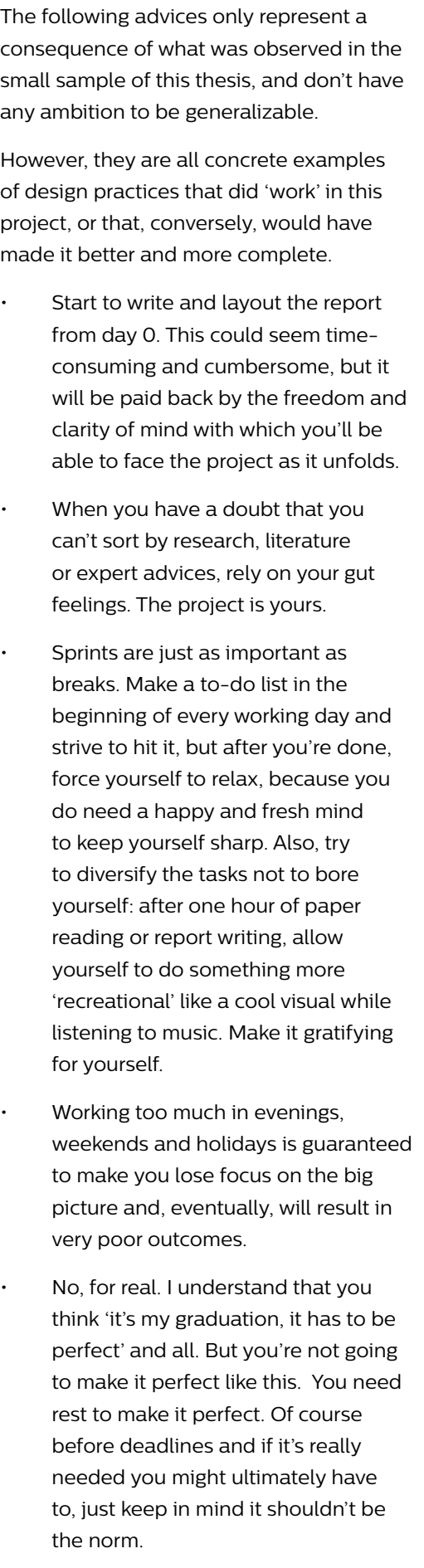 & 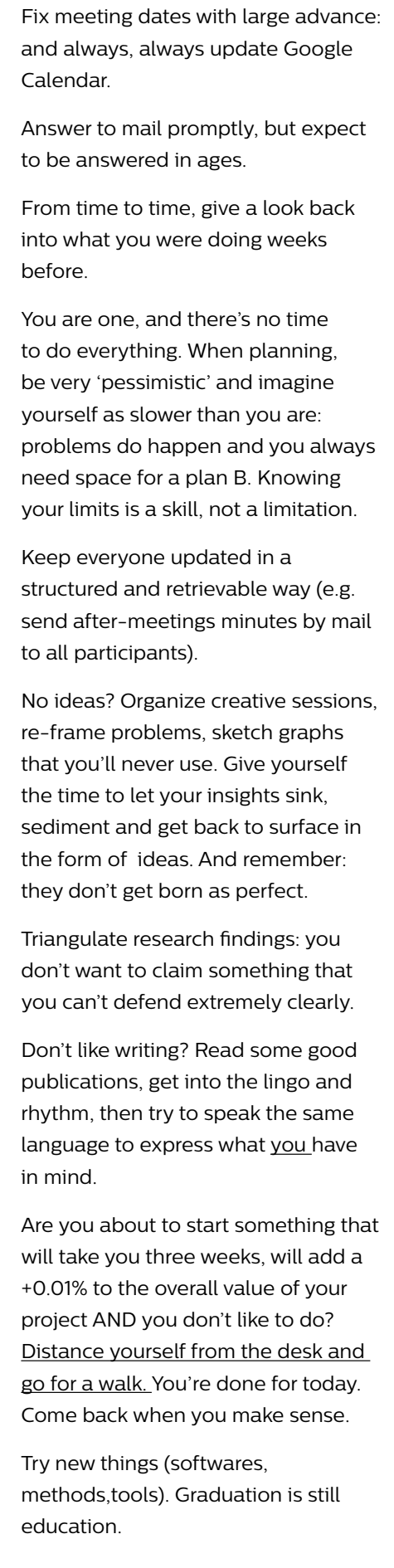 & 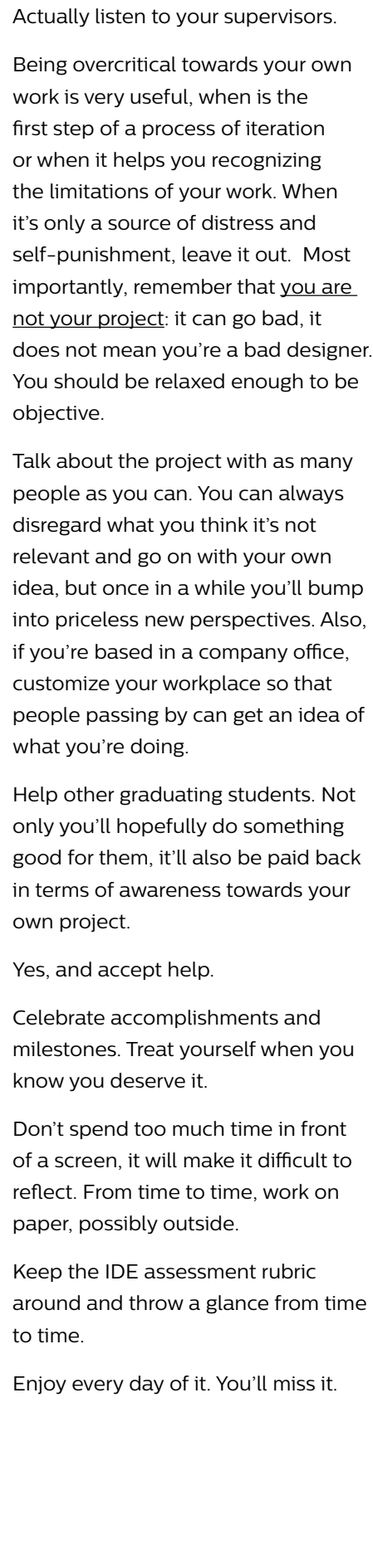 \\
\hline
\end{tabular}


How to empower nurses

to give their best to every

patient?

How to fix information

while saving a life?

How to optimize rescue

data collection and sharing?

Discover the outcomes of

this graduation project,

a challenge to make the job

of ambulance nurses easier,

faster and more accurate,

by enabling hands-

and eyes-free interactions.

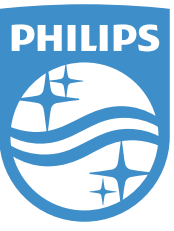

\section{T̛UDelft}

Master Thesis

Design for Interaction

Specialization Medisign

Valeria Pannunzio

Faculty of Industrial Design Engineering

Delft University of Technology 